\title{
Studying the Dynamics and Inhibition of Vimentin Assembly by Small-Angle X-ray Scattering
}

\author{
Dissertation \\ zur Erlangung des mathematisch-naturwissenschaftlichen \\ Doktorgrades \\ „Doctor rerum naturalium" \\ der Georg-August-Universität Göttingen \\ im Promotionsprogramm Physik \\ der Georg-August University School of Science (GAUSS) \\ vorgelegt von \\ Gerrit Frederik Brehm \\ aus Oldenburg (Oldb)
}

Göttingen, 2021 


\section{Thesis Committee:}

Prof. Dr. Sarah Köster

Physics Department

Institute for X-Ray Physics

Georg-August-Universität Göttingen

Prof. Dr. Tim Salditt

Physics Department

Institute for X-Ray Physics

Georg-August-Universität Göttingen

Prof. Dr. Thomas Burg

Department of Electrical Engineering and Information Technology

Technische Universität Darmstadt 


\section{Members of the Examination Board:}

Referee: Prof. Dr. Sarah Köster

Physics Department

Institute for X-Ray Physics

Georg-August-Universität Göttingen

$2^{\text {nd }}$ referee: Prof. Dr. Tim Salditt

Physics Department

Institute for X-Ray Physics

Georg-August-Universität Göttingen

\section{Further members of the Examination Board:}

Prof. Dr. Thomas Burg

Department of Electrical Engineering and Information Technology

Technische Universität Darmstadt

PD Dr. Alexander Egner

Laser Laboratory Göttingen

Prof. Dr. Simone Techert

Physics Department

Institute for X-Ray Physics

Georg-August-Universität Göttingen

Prof. Dr. Stefan Klumpp

Physics Department

Institut für Dynamik komplexer Systeme

Georg-August-Universität Göttingen

\section{Date of Oral Examination:}

04.03.2021 



\section{Contents}

List of Abbreviations $\quad \mathrm{v}$

1 Introduction 1

2 State of the Art 5

2.1 Architecture and Assembly of Intermediate Filaments . . . . . . . . . 5

2.2 Assembly Inhibition and Disassembly of Intermediate Filaments Induced by Small Molecules . . . . . . . . . . . . . . . . . . . . . 8

2.3 Experimental Techniques to Investigate Intermediate Filaments . . . . . . 10

3 Fundamentals 13

3.1 X-Ray Interactions with Matter . . . . . . . . . . . . 13

3.1 .1 Photoelectric Absorption . . . . . . . . . . . . . . 16

3.1 .2 Thomson Scattering . . . . . . . . . . . . . . . . . 17

3.1 .3 Compton Scattering . . . . . . . . . . . . . . 18

3.1.4 Contrast in X-Ray Experiments . . . . . . . . . . . . . . . . 19

3.2 General Principles of Small-Angle X-Ray Scattering . . . . . . . . . . 20

3.2 .1 Form Factor . . . . . . . . . . . . . . . 21

3.2 .2 Structure Factor . . . . . . . . . . . . . . . 23 
Contents

3.2.3 Guinier's Approximation, Pair Distance Distribution Function and Radius of Gyration . . . . . . . . . . . . . . . . . . 24

3.3 Microfluidics . . . . . . . . . . . . . . . . . . . . . 26

3.3.1 Flow at Small Length Scales . . . . . . . . . . . . . . . . . . . 26

3.3 .2 Diffusive Mixing . . . . . . . . . . . . 28

3.4 Cyclic Olefin Copolymer for Microfluidics and Small-Angle X-Ray Scattering 29

3.5 Visible Light Fluorescence Microscopy . . . . . . . . . . . . . . . . . 31

3.5.1 Basic Principles of Fluorescence . . . . . . . . . . . . . . . 31

3.5 .2 Epifluorescence Microscopy . . . . . . . . . . . . . . 33

3.6 Finite Element Method . . . . . . . . . . . . . . . . . . . . . . . 34

4 Experimental Methods $\quad 37$

4.1 Sample Preparation . . . . . . . . . . . . . . . . . . . . 37

4.1 .1 Vimentin Reconstituition . . . . . . . . . . . . . . 37

4.1 .2 Vimentin Labeling . . . . . . . . . . . . . 38

4.1 .3 Vimentin Assembly . . . . . . . . . . . . . . . . . . . . 39

4.2 Cyclic Olefin Copolymer Microfluidic Step Devices . . . . . . . . . . . . 40

4.2 .1 Device Manufacturing . . . . . . . . . . . . . . . . 41

4.2.2 Establishing Flow Connections with Microfluidic Devices . . . . . 44

4.2.2.1 Functional Principle of the Device Holder . . . . . . . 45

4.2.2.2 Device Holder Assembly . . . . . . . . . . . . . . 46

4.3 Small-Angle X-Ray Scattering . . . . . . . . . . . . . . . . 48 48

4.3.1 Experimental Setup at cSAXS Beamline . . . . . . . . . . . . 49

4.3.2 In-House Small-Angle X-Ray Scattering Setup . . . . . . . . . 50 
Contents

4.3.3 Data Acquisition . . . . . . . . . . . . . . . . . . . . 51

4.3.3.1 Static Small-Angle X-Ray Scattering . . . . . . . . . . . 51

4.3.3.2 Time-Resolved Scanning Small-Angle X-Ray Scattering 52

4.3 .4 Data Treatment . . . . . . . . . . . . . . . . . 52

4.4 Fluorescence Imaging of Vimentin Assembly . . . . . . . . . . . . . . . 54

5 Results $\quad 55$

5.1 Cyclic Olefin Copolymer as an X-Ray Compatible Material for Microfluidic

Devices .......................... 56

5.1 .1 Preamble ...................... 56

5.1 .2 Abstract. ....................... 57

5.1 .3 Introduction .................... 57

5.1 .4 Materials and Methods . . . . . . . . . . . . . . 58

5.1.4.1 Device Fabrication and Usage . . . . . . . . 58

5.1.4.2 Protein Purification and Assembly ... . . . . . . 60

5.1.4.3 Small-Angle X-Ray Scattering . . . . . . . . . . . 60

5.1.4.4 Data Analysis . . . . . . . . . . . . . 60

5.1.4.5 Flow Simulations . . . . . . . . . . . . . . 61

5.1 .5 Results and Discussion . . . . . . . . . . . . . . . 61

5.1.5.1 Device Characterization . . . . . . . . . . . . . 61

5.1.5.2 Protein in Flow . . . . . . . . . . . . . . 62

5.1 .6 Conclusion ...................... 63

5.1 .7 Acknowledgements . . . . . . . . . . . . . . . . 63

5.1 .8 References ...................... 63

5.1 .9 Supplementary Information $(\mathrm{SI}) \ldots \ldots \ldots 65$

5.1.9.1 Device Fabrication . . . . . . . . . . . . . . 65 
Contents

5.1.9.2 Cross Section of the Channel Structure . . . . . . . . 67

5.1.9.3 Sample Holder . . . . . . . . . . . . . . . . . . 68

5.2 Microfluidic Step-Devices for X-Ray Measurements . . . . . . . . . . . . 69

5.2.1 Finite Element Method Simulations . . . . . . . . . . . . . . . 69

5.2.1.1 Mesh Size Dependency . . . . . . . . . . . . . 71

5.2.1.2 Flow Profiles and Accessible Assembly Time Points . . . 73

5.2.2 Correction Factor Calculation . . . . . . . . . . . . 76

5.2 .3 Streak Finding Algorithm . . . . . . . . . . . . . . . 77

5.2.4 Time-Resolved Vimentin Assembly . . . . . . . . . . . . . . . . 79

5.3 Small Molecules Inhibiting Vimentin Assembly . . . . . . . . . . . . . 82

5.3.1 Analysing Assembly Kinetics . . . . . . . . . . . . . . . . . . 82

5.3.1.1 Structure-Detecting Algorithm . . . . . . . . . . 83

5.3.1.2 Benchmarking ................ . 84

5.3.2 Vimentin Assembly in the Presence of N-Acetyl-L-Cysteine . . . 86

5.3.2.1 Length Distributions . . . . . . . . . . . . . 86

5.3.2.2 Aggregate Distributions . . . . . . . . . . . . . 90

5.3.2.3 Molecular Mass and Radius of Gyration . . . . . . . . 92

5.3.3 Vimentin Assembly in the Presence of Sodium Phenylbutyrate . . 95

5.3.3.1 Length Distributions . . . . . . . . . . . . . . . 95

5.3.3.2 Aggregate Distributions . . . . . . . . . . . . . 98

5.3.3.3 Molecular Mass and Radius of Gyration . . . . . . . . 100

5.3.4 Comparison of the Vimentin Assembly in the Presence of $\mathrm{N}$ Acetyl-L-Cysteine and Phenylbutyrate . . . . . . . . . . 102

5.3.4.1 Fluorescence Microscopy Data . . . . . . . . . . . . . 102

5.3.4.2 Static Small-Angle X-Ray Scattering . . . . . . . . . 106 
6 Discussion 109

6.1 Microfluidic Step Devices for Scanning Small-Angle X-Ray Scattering . 109

6.2 Small Molecules Inhibiting Vimentin Assembly . . . . . . . . . . . . 113

7 Conclusions and Future Experiments 117

$\begin{array}{ll}\text { A Appendix } & 121\end{array}$

A Connecting the Mounted Device and Setting Up the Flow for Microfluidic Scanning Small-Angle X-Ray Scattering Measurements . . . . . . . . . 121

B Sodium Phenylbutyrate Data Sets . . . . . . . . . . . . . . . . . 123

B.1 Data Set $2 \ldots \ldots \ldots \ldots \ldots \ldots \ldots \ldots$

B.2 Data Set $3 \ldots \ldots \ldots \ldots \ldots \ldots \ldots \ldots$

C N-Acetyl-L-Cysteine Data Sets . . . . . . . . . . . . . . . . . . . 127

C.1 Data Set $2 \ldots \ldots \ldots \ldots \ldots \ldots$. . . . . . . . . . . . . . . . .

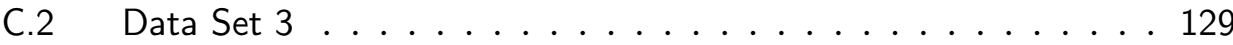

Bibliography 131

Danksagung - Acknowledgments 143

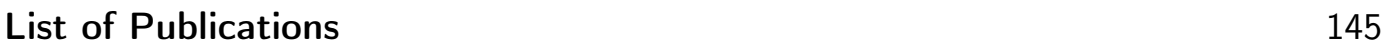





\section{Abbreviations}

$\begin{array}{ll}\text { ACC } & \text { N-Acetyl-L-cysteine } \\ \text { CMOS } & \text { Complementary metal-oxide-semiconductor } \\ \text { COC } & \text { Cyclic olefin copolymers } \\ \text { CSAXS } & \text { Coherent Small-Angle X-ray Scattering } \\ \text { D } & \text { Diffusion coefficient } \\ \text { FEM } & \text { Finite element method } \\ \text { FWHM } & \text { Full width at half maximum } \\ \text { NOA } & \text { Norland Optical Adhesive } \\ \text { PBA } & \text { Sodium phenylbutyrate } \\ \text { pddf } & \text { Pair-distance distribution function } \\ \text { PDE } & \text { Partial differential equations } \\ \text { PDMS } & \text { Polydimethylsiloxane } \\ \text { PE } & \text { Polyethylene } \\ \text { Pe } & \text { Péclet number } \\ \text { Re } & \text { Reynolds number } \\ \text { SAXS } & \text { Small-angle X-ray scattering } \\ \text { TRIS } & \text { Tris(hydroxymethyl)aminomethan } \\ \text { ULF } & \text { Unit-length filament } \\ \text { UV } & \text { Ultraviolet-visible }\end{array}$





\section{Introduction}

Eukaryotic cells have to fulfil a large variety of tasks including providing structure, transporting molecules and exerting forces. One protein system expressed to meet these requirements is the cytoskeleton. The cytoskeleton is a complex network comprised of three primary components; microfilaments with diameters $\sim 8 \mathrm{~nm}$, microtubules with outer diameters of $\sim 25 \mathrm{~nm}$, and intermediate filaments (IFs) with intermediary diameters of 8-10 $\mathrm{nm}[1,2]$.

Whereas actin microfilaments and microtubules are ubiquitous in all eukaryotic cells, intermediate filaments describe a class of filaments, with the exact type varying from cell to cell, e.g., keratin IFs are found in hair cells [3]. Neurofilaments are present along the axons of vertebrate neurons [1]. Desmin plays a vital part in muscle cells [4]. Collectively, these components are important for mechanical properties of the cell, they mediate cytoskeletal cross-talk, influence cell migration, cell contraction, cell signaling and are critical for cell division and maintaining the cell shape [1,5-8]. Despite the specific structure and function of each component, there is evidence that only the interplay between components enable complex processes like cell migration [9]. Three components of the cytoskeleton for NIH3T3 cells are shown in Fig. 1.1.

IF networks specifically play a critical role in a variety of cellular processes, e.g., 

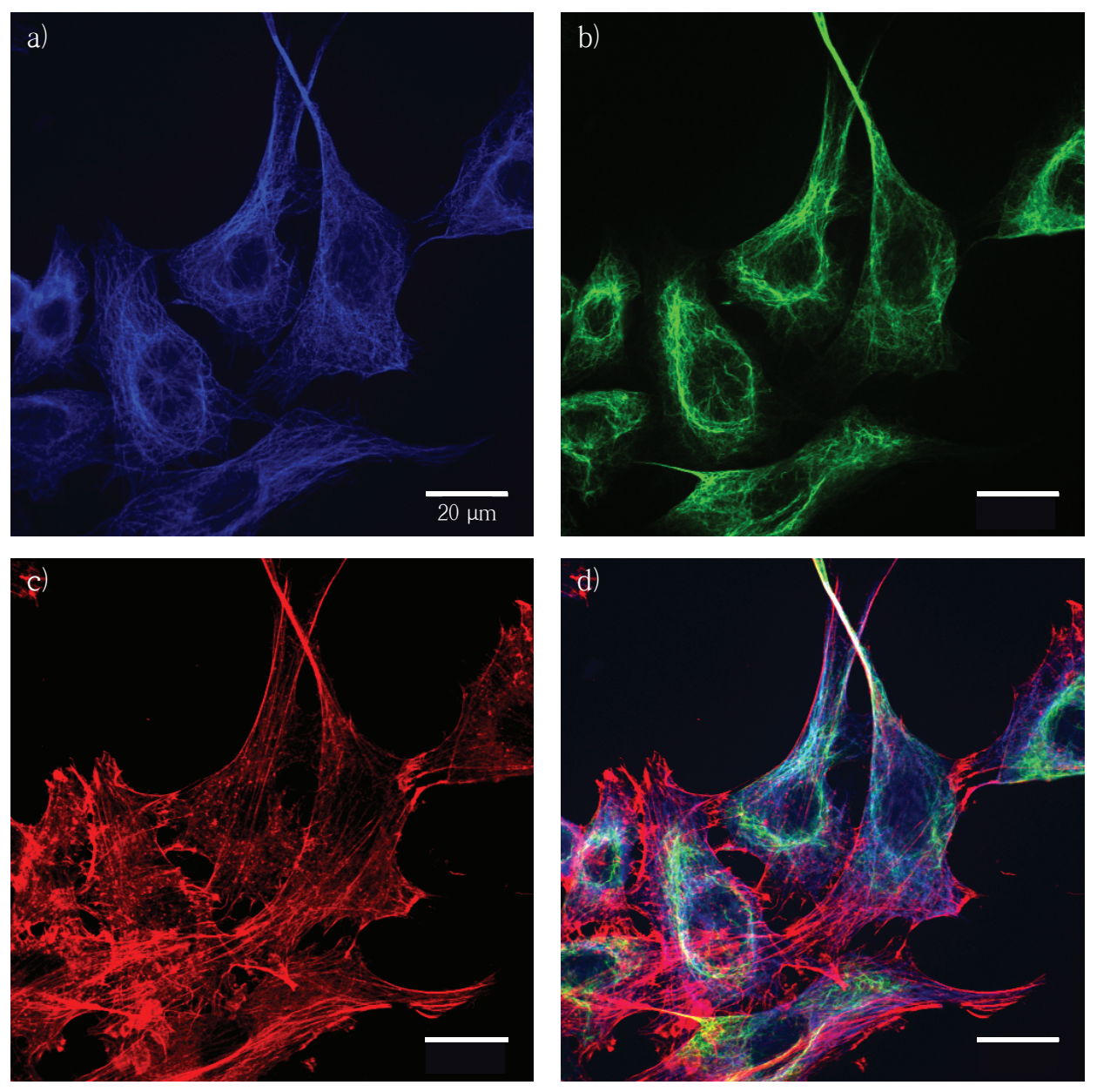

Figure 1.1.: Fluorescence confocal images of NIH3T3 fibroblast cells. a) Microtubules are shown in blue, b) vimentin IFs green and c) actin microfilaments in red. d) Composite overlay image. Images courtesy of Dr. Ulrike Rölleke (Institut für Röntgenphysik, Universität Göttingen).

mechanical structure and cell organelle positioning [1]. A better understanding of the structure and molecular mechanisms during IF assembly is of high relevance, as IFs are furthermore involved in the pathology of severe diseases, e.g., lung cancer and Alexander disease [10-12]. One approach to further understand the occurring interactions, is to investigate isolated single components.

In this work, the role of two chemical compounds and their role in the vimentin assembly process is investigated. Different experimental techniques are combined to capture the relevant dynamics at different length scales. Small-angle X-ray scattering (SAXS) is used to access structural parameters on the nanometre scale. Combined with microfluidics and finite element method (FEM) simulations, 
scanning-SAXS is further employed to access the related assembly dynamics. Fluorescence microscopy enables investigation of filament formation on the micrometer scale.

In chapter 2 an overview of the current literature on vimentin is given and the research question of this thesis is motivated. The theoretical background for the methods used is presented in chapter 3. Chapter 4 explains the methods used, the protocols used and developed and the steps for data acquisition. The measured data are presented and analysed in chapter 5 and discussed in chapter 6 . Chapter 7 concludes with a summary and possible future experiments. 



\section{State of the Art}

In this chapter, the relevance of the biological system under investigation and current research approaches are introduced. Remaining open questions are addressed. The selected projects are contextualized in this chapter and the techniques used are introduced.

\subsection{Architecture and Assembly of Intermediate Filaments}

More than 60 different genes are known to encode for IF proteins, showing the versatility of IFs. IF genes are among the 100 largest gene families in humans [13]. This versatility enables IF proteins to fulfil various roles, e.g., influencing cell migration and the mechanical properties of the cell [1]. These roles are fulfilled throughout different parts of epithelial biology: in the cytoplasm, in hair, nails and in stress sensing $[1,14]$.

Depending on their sequence structure, IFs are assigned to one of five different classes [6]: Keratins (types I and II), vimentin, glial fibrillary acidic protein, desmin and peripherin (type III), neurofilaments (type IV) and nuclear lamins (type V) [15]. Despite their versatility, IFs share a common secondary structure comprised of three domains. A central $\alpha$-helical rod domain consists of several 


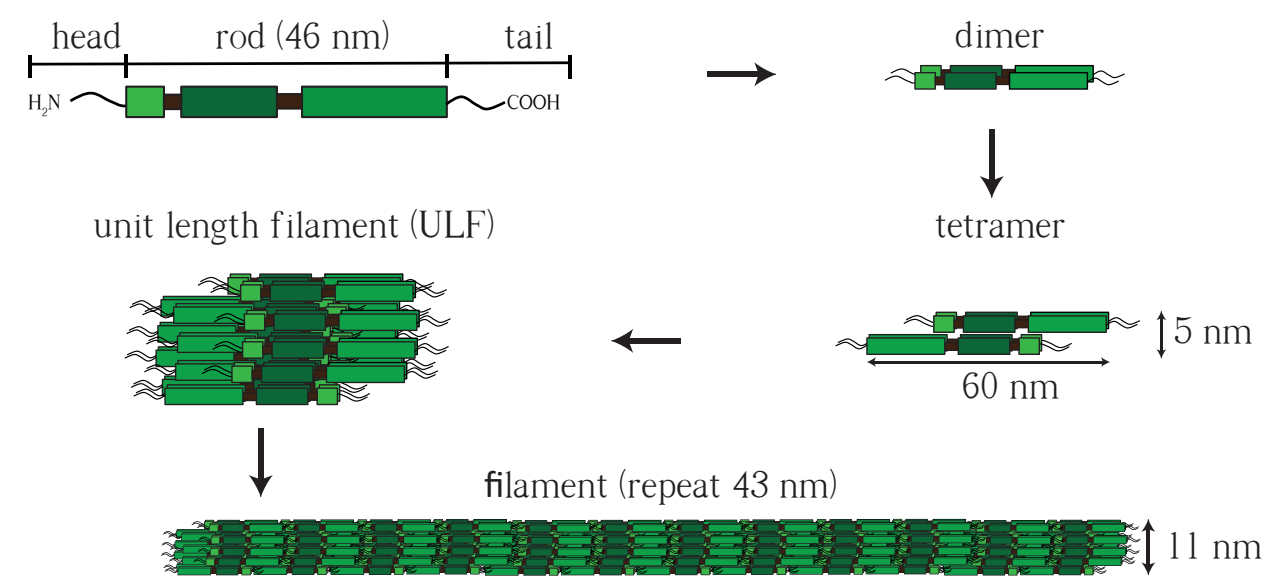

Figure 2.1.: Schematic representation of vimentin assembly. Vimentin monomers contain a central $\alpha$-helical rod domain with disordered a head and tail. A parallel dimer is formed out of two monomers. Anti-parallel, half-staggered assembly of two dimers yields a tetramer, which subsequently laterally assembles with seven other tetramers to form a ULF. ULFs anneal longitudinal to an extended filament (sketches not to scale). Figure with minor adaptations from [22] under the Creative Commons Attribution (CC BY) license.

$\alpha$-helical segments joined by linkers which connects to the disordered head and tail. IFs share the hierarchical process of assembling into larger structures [16]. The hierarchical assembly for vimentin, the most frequently expressed type III IF [17], is subsequently described.

The assembly process for vimentin is schematically shown in Fig. 2.1. The hierarchical assembly of vimentin IFs starts with two rod shaped monomers which associate laterally into a parallel coiled-coil dimer. Subsequently, the anti-parallel, halfstaggered assembly of two dimers yields a non-polar tetramer [18]. This process is favored by the periodical arrangement of positive and negative charges on the central rod domain of the monomers, which induce electrostatic interactions [19]. Further lateral assembly of tetramers, induced by, e.g., changing the ionic strength of the surrounding medium, forms unit-length filaments (ULFs). ULFs form the final filament by longitudinal end to end assembly [16, 20,21]. In vimentin, a subsequent compaction step occurs, reducing the filament diameter from $17 \mathrm{~nm}$ to $11 \mathrm{~nm}$. It is important to note that throughout the assembly process the vimentin tetramer configurations are dynamic $[18,23]$. Despite the hierarchical assembly for vimentin ULFs, tetramer numbers per ULF are influenced by the surrounding ion type and ion concentration [22, 24, 25]. For vimentin, on average, 32 monomers are 
present in one ULF. However, depending on the ionic strength during assembly, the number of monomers can increase by over $400 \%$ per ULF [22]. Furthermore, the assembled filament remains a dynamical structure, as subunit exchanges of monomers in assembled filaments occur and mechanics are dependent on $\mathrm{pH}$ and ion charges [26, 27]. An important role in inducing conformational changes during vimentin assembly is fulfilled by disulphide cross-links between cysteine residues of wild type vimentin. Whereas cysteine deficient vimentin C328S can assembly to filaments, heavy cross-linking in wild type vimentin induced by mutations or chemical compounds, such as diamide, result in conformational changes. These changes ultimately inhibit the formation of filaments, as the resulting structures are incompatible for IF assembly [28, 29].

Type III IFs form a dynamic network, extending from the nucleus to the cell membrane (see Fig. 1.1b for a vimentin network in NIH3T3 fibroblast cells). The IF network modulates and interacts with the remaining components of the cytoskeleton, actin microfilaments and microtubules [30,31]. The IF network plays a critical role in a variety of cellular processes, including cell organelle positioning, deformability of cells, cell division and nucleus integrity under stress [17, 32-34]. Understanding the mechanisms of assembly, network formation and interaction, as well as occurring variations for type III IFs, is of major importance, as type III IF are involved in the pathology of severe diseases, e.g., cancer [11], Alzheimer disease and multiple sclerosis [8]. Extensive research for various topics related to type III IFs both in vivo and in vitro is performed and elaborate reviews are available $[8,14,35]$. Vimentin is often investigated as a role model as it is the most expressed type III IF [17].

Various approaches are used to investigate the functions and alterations of vimentin. Post-translational modifications are often induced by enzymes in cells to alter IFs in specific situations, e.g., phosphorylation of vimentin is critical for the completion of cytokinesis [36]. Kraxner et al. recently investigated the effect of phosphorylation on single vimentin filaments and reported a softening of the IF [37].

An other approach is to mutate selected amino acids in the amino acid sequence of vimentin and to observe the occurring effects, e.g., on the vimentin assembly [38]. Brennich et al. exchanged the tyrosine at position 117 of the vimentin amino acid sequence to leucine. This changes the diameter of the vimentin ULFs after lateral association, but furthermore inhibits the formation of filaments by longitudinal elongation [39]. 
A third approach is the modification of the environment around the IF by changing, e.g., the ionic strength or the addition of chemical compounds. In cells, this environmental change occurs, e.g., by oxidative or heat induced stress [40]. When experiencing heat induced stress cells express heat shock protein (HSP) 27 [41]. This protein can interact with IFs and stabilise the vimentin network [42, 43]. The general importance of this interaction is further expressed by mutations in HSPs, inducing IF aggregation and phenotypes similar to IF disorder related diseases [44].

The third approach is of particular interest in modern medicine as chemical agents can be injected into the human body. Severe diseases caused by vimentin IF mutations are treated with a range of small molecules, and a better understanding of the mechanisms involved could improve treatment. Therefore, one project in this thesis is investigating the effect of two common compounds used to treat severe diseases associated with vimentin IFs on vimentin assembly in vitro.

\subsection{Assembly Inhibition and Disassembly of Intermediate Filaments Induced by Small Molecules}

Three different chemical compounds are presented in the following. The first chemical compound, diamide, serves as an example for which the interaction process with vimentin is known. The second and third chemical compounds, N-Acetyl-L-cysteine (NAC or ACC, ACC in the following) and sodium phenylbutyrate (PBA), are used in the treatment of various diseases related to vimentin IFs. The effect of ACC and PBA on purified vimentin during assembly is investigated in this work and the results are presented in Sec. 5.3.2 and Sec. 5.3.3, respectively. Diamide is an electrophilic compound which facilitates disulphide bond formation by forming adducts with thiol groups of cysteine residues [29, 45]. The formation of extensive disulphide bonds inhibits vimentin IF formation in wild type vimentin [28, 29], see Fig. 2.2. Interestingly, inhibition does not occur in cysteine deficient vimentin mutant $\mathrm{C} 328 \mathrm{~S}$. This indicates that diamide induced disulphide bond formation between cysteine residues in vimentin tetramers results in nonproductive conformations for subsequent IF assembly [28, 29].

The addition of diamide to preassembled vimentin wild type IFs does not disrupt the formed IFs in vitro, indicating that additional disulphide bond formation in assembled IFs can not occur or occurs without disrupting the IFs [29]. The 
Control
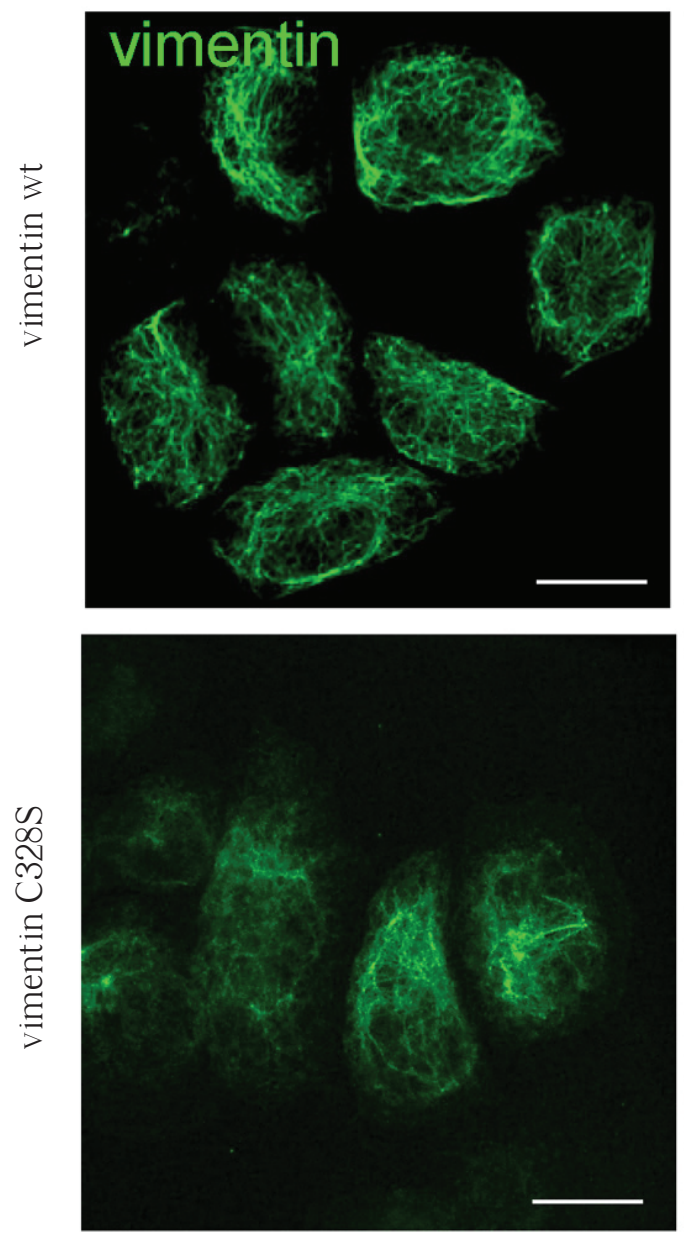

Diamide
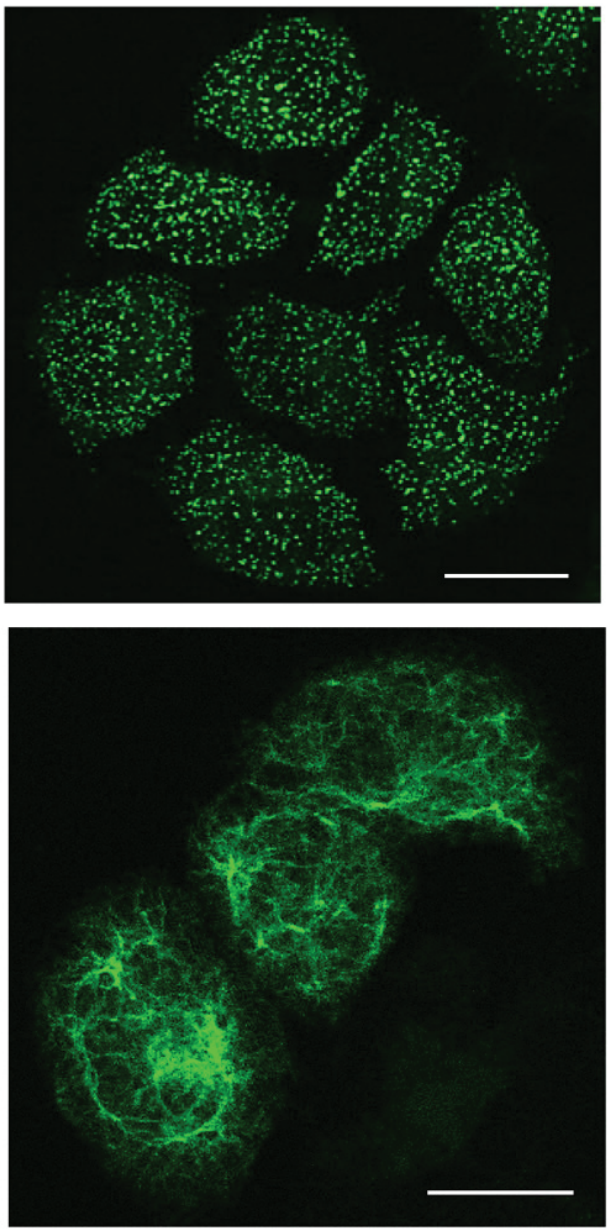

Figure 2.2.: Effect of electrophilic agents on vimentin organization in cells. SW13/cl.2 cells stably transfected with RFP//vimentin wt or Cys328Ser (C328S) were treated with $1 \mathrm{mM}$ diamide for $15 \mathrm{~min}$ for $2 \mathrm{~h}$, after which, cells were fixed and the vimentin network was visualized by immunofluorescence. Scale bars, $20 \mu \mathrm{m}$. Caption and figure with minor adaptations from [29] under the Creative Commons Attribution (CC BY-NC-ND 4.0) license. 
importance of the C328 residue, investigated in this study, on vimentin assembly is further emphasized by subtle differences in the filament radius between the vimentin IFs formed by the C328S mutant compared to the vimentin wild type [29]. In cells, diamide induces reorganization on vimentin IFs into discrete dots, resulting into a major disruption of the vimentin IF network (see Fig. 2.2) [29, 33].

PBA is an aromatic fatty acid widely used in medical applications. It is used in urea cycle disorder due to its ammonia scavenger function after oxidation in the liver [46]. Furthermore, potent anti-tumor effects are reported in vitro [47, 48], as well as applications in the treatment of Alzheimer's disease, due to its counteraction of protein aggregation [49]. The antagonizing of protein aggregation is further reported for types I and II IF keratin [50] and the type III IF desmin [51]. When plectin-deficient mice were treated with PBA, PBA acted as a chaperone, resulting in the reduction of desmin aggregates and an increase in muscle functionality was observed [51].

ACC is known for its antioxidant and disulphide breaking capabilities. It is used in a fast variety of treatments, e.g., in cystic fibrosis and acetaminophen (active substance in Paracetamol) poisoning $[52,53]$. In cystic fibrosis, mucin polymers, the proteins which form mucus via disulphide bonds between cysteine-rich $\mathrm{N}$ and $\mathrm{C}$ domains, are more cross-linked compared to mucin in healthy lungs. As a result, the mucus is highly elastic, harder to transport and accumulates in the lungs, leading to airflow obstruction and lung infections [52]. ACC breaks these disulphide bonds by binding with its own thiolate group to the central sulphur, releasing the other thiolate group [52]. This reaction occurs as the nucleophilicity of ACC greater than the nucleophilicity of cysteine [54].

\subsection{Experimental Techniques to Investigate Intermediate Filaments}

Similar to the different approaches to study IFs, the methods employed to observe IFs are manifold as IFs dynamics occur on length scales of a few nm (first steps of assembly) to $\mu \mathrm{m}$ (filament networks in cells), and time scales between ms (first steps of assembly) and minutes (network formation). Techniques with a high spatial resolution, e.g., electron microscopy, atomic force microscopy [5] and 
SAXS [55], require, in general, acquisition times on the order of seconds, thus limiting the time-resolution achievable. Techniques capable of achieving smaller time resolution, e.g., fluorescence microscopy, can be diffraction-limited in resolution. To overcome these limitations and to provide controlled chemical environments, various techniques are combined with microfluidics [27, 56-58].

Microfluidic devices are referred to as "lab-on-a-chip" [59], originating from the possibility to incorporate different processes in one device. Microfluidics allow for precise manipulation of microliter sample volumes by, e.g., mixing fluids, changing chemical environments and reaction times [60]. In this thesis microfluidics are combined with SAXS, as the investigated assembly process of vimentin tetramers to ULFs occurs in the time range of $\mathrm{ms}$ to $\mathrm{s}$ and the relevant length scales are the order of $\mathrm{nm}$. The distinct control over the ion concentration inducing the assembly, temporal resolution and observable length scale is simultaneously realized by combining microfluidics and SAXS.

A detailed overview of combining microfluidics with SAXS in a time-resolved manner is given in the published work 'Cyclic olefin copolymer as an X-Ray compatible material for microfluidic devices' (see Sec. 5.1.3). 



\section{Fundamentals}

In this chapter, the theoretical background for the methods used is presented. The basic interactions of X-rays with matter are addressed. Furthermore, the fundamentals for microfluidics, fluorescence microscopy and finite element simulations are described. The descriptions given are based on [61-66]

\subsection{X-Ray Interactions with Matter}

Electromagnetic radiation can interact with matter in several ways. Depending on the incident photon energy $E$, expressed by the Plank-Einstein relation $E=h c / \lambda$, where $h$ represents the Planck's constant, $\lambda$ the wavelength and $c$ the speed of light in vacuum, either elastic or inelastic scattering can occur. Interactions of electromagnetic radiation with matter depends on the energy $E$ of the incident photon and the atomic mass $u$. In the case of elastic scattering, no energy loss occurs between the incident and the scattered photon, thus the wavelength $\lambda$ is constant. For inelastic scattering events there is an energy loss. This loss results in the scattered photon to have a larger wavelength $\lambda$ than the incident photon. Examples for elastic scattering are Rayleigh and Thomson scattering, whereas Compton scattering describes an inelastic scattering process. Photoelectric absorption and pair 


\section{\begin{tabular}{l|l} 
Chapter 3 & FUNDAMENTALS
\end{tabular}}

production are examples for photon absorption. Predominant effects of interaction between photon energies used for X-ray diffraction $(\approx 10 \mathrm{keV})$ and matter, namely photoelectric absorption, Thomson and Compton scattering, are discussed in the following.

Measuring the intensity $I(z)$ of a photon beam with intensity $I_{0}$ before and behind matter with thickness $z$ reveals a reduction of the measured intensity $I(z)$. The reduction in intensity $d I$ is called attenuation and originates from photon interactions with matter. The probability for interaction of the incident photons with one atom of the matter is dependent on the photon energy and the type of atom and is given by the cross-section $\sigma_{a}\left[\mathrm{~cm}^{2}\right]$. The attenuation is described by

$$
d I=-\sigma_{a} n I_{0} d z
$$

where $n$ is the number of atoms per unit volume. By integrating Eq. 3.1 from $z_{\min }=0$ to $z_{\max }=z$ we obtain the Lambert-Beer law

$$
I(z)=I_{0} e^{(-\mu z)}
$$

where $\mu=\sigma \times \mathrm{n}\left[\mathrm{cm}^{-1}\right]$ is the total linear attenuation coefficient. The mass attenuation coefficient $\left(\frac{\mu}{\rho}\right)\left[\mathrm{cm}^{2} / \mathrm{g}\right]$ is obtained by dividing the total linear attenuation coefficient $\mu$ by the density $\rho$ of the corresponding element. The total attenuation is expressed as the sum of all possible interactions and their mass attenuation coefficients:

$$
\left(\frac{\mu}{\rho}\right)_{\text {Total }}=\left(\frac{\mu}{\rho}\right)_{\text {Photoelectric }}+\left(\frac{\mu}{\rho}\right)_{\text {Coherent Scat }}+\left(\frac{\mu}{\rho}\right)_{\text {Compton Scat }}+\left(\frac{\mu}{\rho}\right)_{\text {Pair-Prod. }} .
$$

Figure 3.1 shows the individual contributions to the total attenuation by the different interactions for energies ranging from $1 \mathrm{keV}$ to $100 \mathrm{keV}$ for a protein with the empirical formula of $\mathrm{H}_{50} \mathrm{C}_{30} \mathrm{~N}_{9} \mathrm{O}_{10} \mathrm{~S}$ [68]. The total attenuation is shown in purple and one absorption edges is visible at a photon energy of $2 \mathrm{keV}$. For imaging of biological matter energies up to $100 \mathrm{keV}$ are most relevant [61]. 


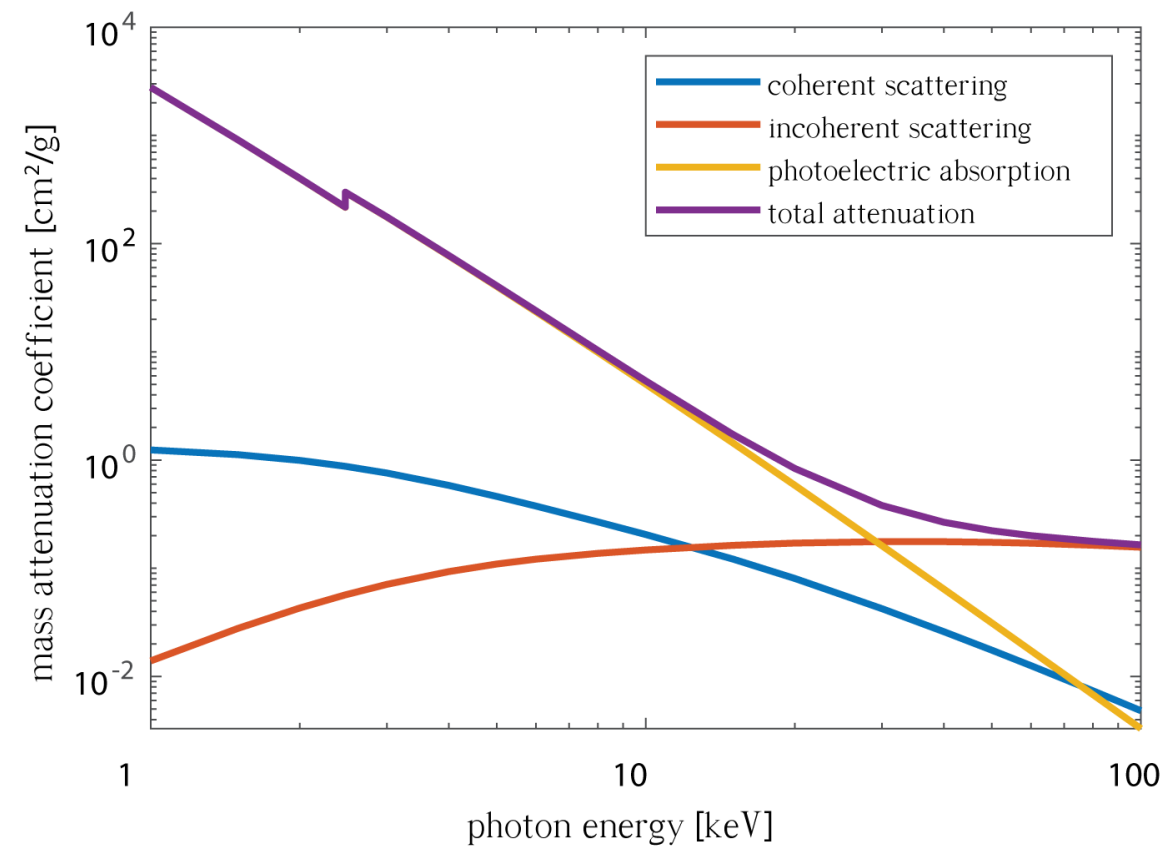

Figure 3.1.: Mass attenuation coefficient for coherent (Thomson) scattering, incoherent (Compton) scattering and photoelectric absorption for an average protein content of cellular material, described by the empirical formula $\mathrm{H}_{50} \mathrm{C}_{30} \mathrm{~N}_{9} \mathrm{O}_{10} \mathrm{~S}$ for energies from $1 \mathrm{keV}$ to $100 \mathrm{keV}$. In this energy range no pair production occurs. Data calculated with [67]. 


\section{\begin{tabular}{l|l} 
Chapter 3 & FUNDAMENTALS
\end{tabular}}

\subsubsection{Photoelectric Absorption}

Photoelectric absorption is the predominant X-ray interaction for photon energies below $100 \mathrm{keV}$ [63]. A representation of the process is depicted in Fig. 3.2. The incident photon with an energy $E_{0}$ is absorbed by an inner shell electron with a binding energy $B_{e}$ lower than the photon energy. The momentum and energy of the incident photon can not be solely transferred to the photoelectron, resulting in the atom taking momentum from the incident photon. Consequently, the atom remains in a photoionizised state. The vacancy in the inner shell exists for a short lifetime $\left(\sim 10^{-14} \mathrm{~s}\right)$ before an electron from an outer shell fills the vacancy [61]. To account for the difference in binding energy, the transitioning electron emits characteristic electromagnetic radiation. Two competing mechanism occur: Fluorescence describes the emission of characteristic photons. Depending on the size of the atom the wavelength of the emitted photon ranges from visible light to energies in the X-ray range. Competing with fluorescence is the Auger effect, where the energy difference is transferred to another electron and is subsequently emitted from the atom. The latter is dominant for light elements up to atomic numbers $Z$ of $Z=30$. The cross-section for photoelectric absorption is expressed

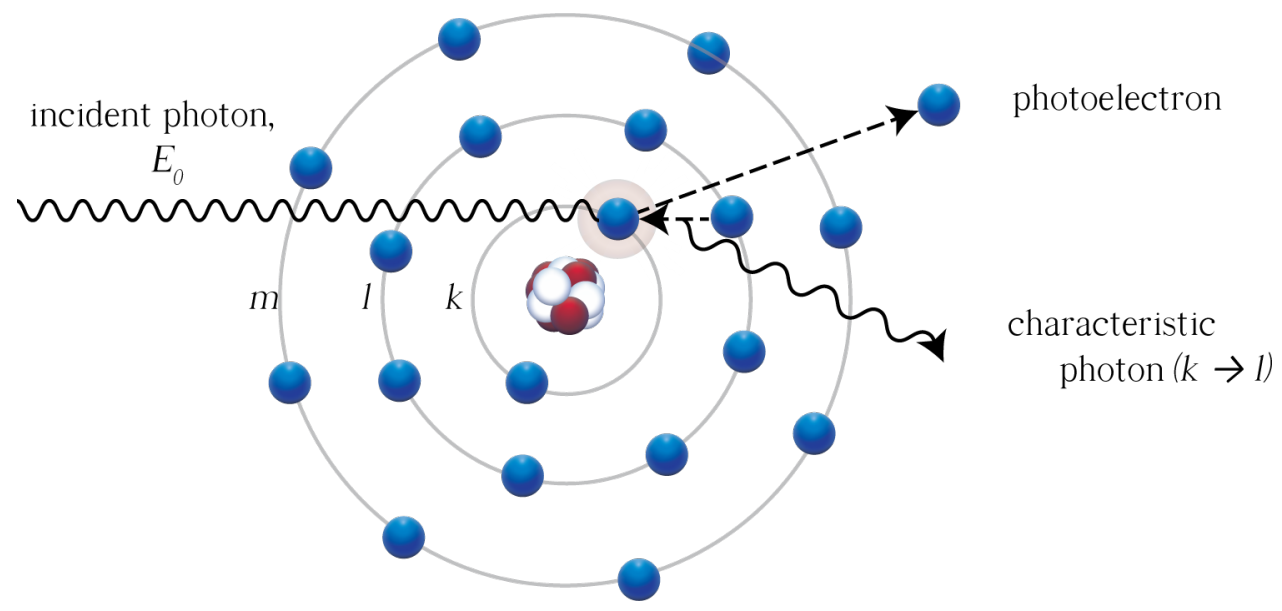

Figure 3.2.: Schematic of photoelectric absorption. The incident photon is absorbed by a bound inner shell electron and a photoelectron is emitted if the incident photon energy exceeds the binding energy of the electron. The vacancy in the inner shell results in an ionized atom. After a short lifetime $\left(\sim 10^{-14} \mathrm{~s}\right)$ the vacancy is filled with an outer shell electron, which emits the difference in binding energy as a characteristic photon[61]. 
by

$$
\sigma_{a}^{p \cdot e} \propto \frac{Z^{n}}{E^{3}}
$$

with $n \approx 4$ for heavy and $n \approx 4.5$ for light elements [61].

\subsubsection{Thomson Scattering}

Thomson scattering describes elastic scattering by point charges and was first described by J.J. Thomson in 1906 [69]. It is the fundamental process for structural investigation of matter with scattered $\mathrm{X}$-ray radiation. A point charge oscillates in the electric field of the incident electromagnetic wave and emits an electromagnetic wave of the exact frequency $v$. Thus, the photon energy remains identical during the interaction process. The energy conservation is shown in Fig. 3.3. As a result, no momentum or drift velocity is transferred to the scattering point charge. Due to the elastic scattering, the scattered electromagnetic waves can interfere with each other on the detector, allowing for structural analysis of the scattering matter by analysing the resulting pattern. The total cross-section $\sigma_{e}^{T h}$ for a single free electron with the electron radius $r_{e}$ in classical electrodynamics is

$$
\sigma_{e}^{T h}=\frac{8 \pi}{3} r_{e}^{2}=0.665 \cdot 10^{-28} \mathrm{~m}^{2}, \quad \text { with } r_{e}=\frac{1}{4 \pi \epsilon_{0}} \frac{e^{2}}{m_{e} c^{2}} \text {. }
$$

Eq. 3.5 reveals the independence of the total cross-section $\sigma_{e}^{T h}$ from the photon energy.

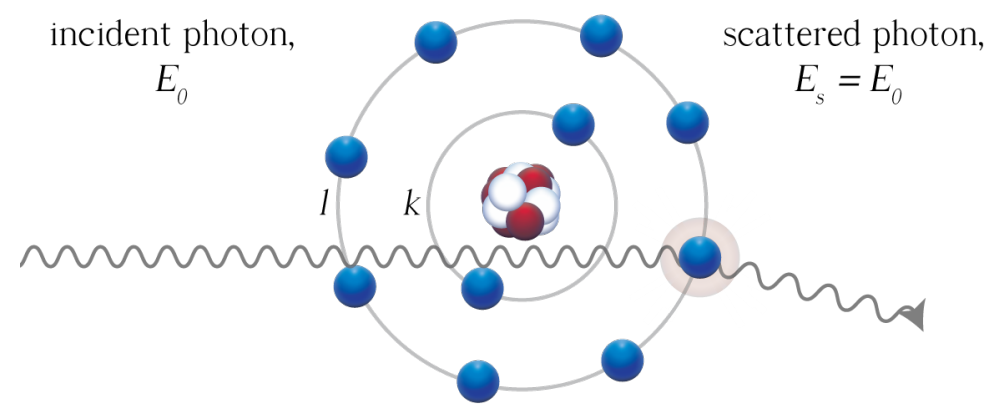

Figure 3.3.: Schematic of Thomson scattering. The incident photon interacts with all outer shell electrons. The scattered photon is emitted with no energy loss. 


\section{\begin{tabular}{l|l} 
Chapter 3 & FUNDAMENTALS
\end{tabular}}

\subsubsection{Compton Scattering}

Compton scattering occurs when the incident photon interacts with an outer-shell electron. It was first described by A.H. Compton in 1923 [70]. The incoherent and inelastic scattering process is depicted in Fig. 3.4. An incident photon with energy $E_{0}=h v_{0}$ interacts with an electron with low binding energy $E_{B}$ compared to the incident photon $\left(E_{B} \ll h v\right)$. Momentum is transferred to the recoiled electron which is consequently expelled from the shell. The scattered photon is emitted at a specific angle $\theta$ and has an increased wavelength, thus offsetting the energy loss $\left(E_{s}=h v^{\prime}<E_{0}\right)$. With the electron mass $m_{e}$ and the speed of light in vacuum $c$, the energy of the scattered photon is given by:

$$
h v^{\prime}=\frac{h v}{1+\frac{h v}{m_{e} c^{2}}(1-\cos \theta)} .
$$

The fact that the attenuation coefficient is proportional to mass density suggest the definition of the so called mass attenuation coefficient.

$$
\left(\frac{\mu}{\rho}\right)_{\text {Comptonscat }} \propto \text { constant. }
$$

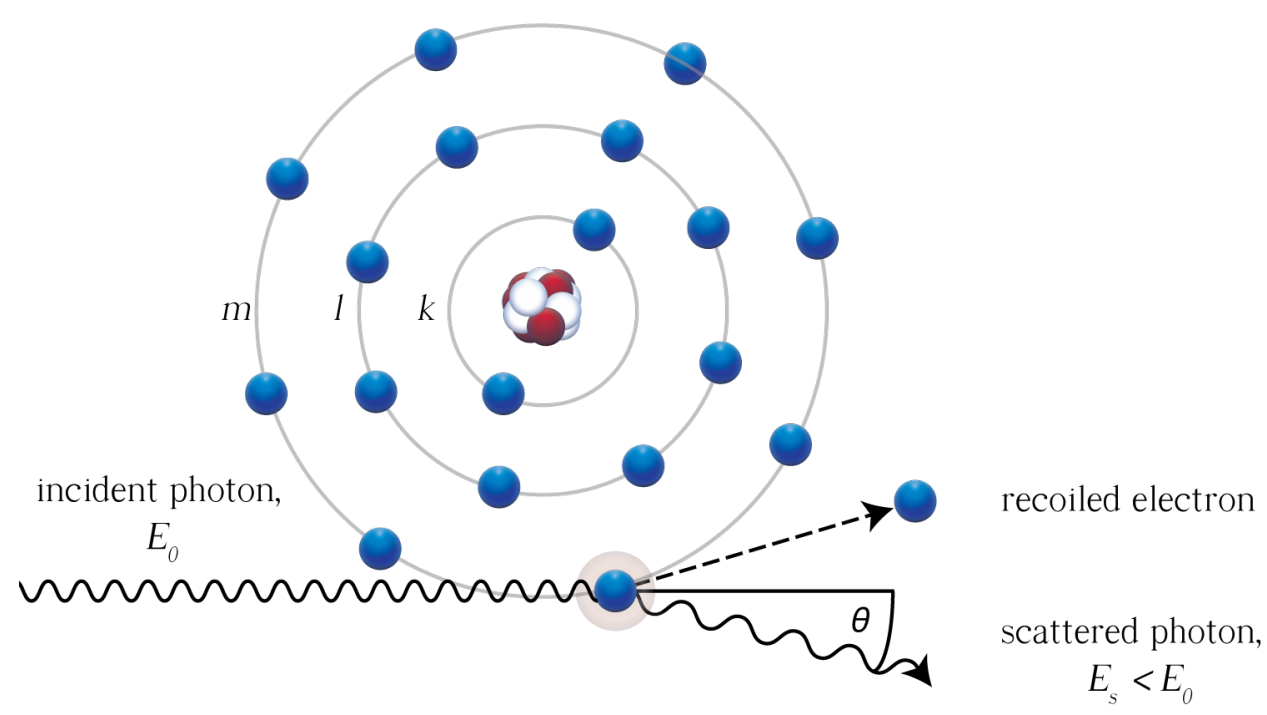

Figure 3.4.: Schematic of Compton scattering. An incident photon with energy $E_{0}$ and the trajectories of the scattered photon and electron. The scattered electron gains kinetic energy and momentum by the interaction with the photon and is in plane with the scattering plane defined by the incident and scattered photon. 
The cross-section of the Compton effect $\sigma_{C}$ is proportional to the atomic number $Z$ [61]

$$
\sigma_{C} \propto Z
$$

Due to the incoherent and inelastic scattering processes, no interference patterns can be observed and therefore no structural information on the scattering matter is gained.

\subsubsection{Contrast in X-Ray Experiments}

The amplitude of scattered X-rays inside a material is determined by the scattering length $f_{\mathrm{X}}$ and correlates to the scattering power of the material. For $\mathrm{X}$-rays the scattering length $f_{\mathrm{X}}$ is defined as:

$$
f_{\mathrm{X}}=N_{e^{-}} * r_{0},
$$

with $r_{0}$ as the classical electron radius and $N_{e^{-}}$the number of electrons per atom in the respective element. Equation 3.9 implies an increase in the scattering amplitude for atoms with increasing numbers of electrons $e^{-}$, e.g., for increasing atomic numbers Z . Elements common in biological matter together, with their atomic mass, number of electrons and the resulting scattering length, are displayed in Tab. 3.1. Increasing atomic mass $u$ does not necessarily result in a larger scattering length, e.g., Deuterium has the same scattering length as Hydrogen, while having double the atomic mass.

Biological matter consists of atoms with low atomic number. Compared to heavy atoms like metals, the scattering amplitude is low. Biological samples are often measured in aqueous solution which contains atoms of similar atomic number as the specimen, resulting in low contrast and the necessity of background

Table 3.1.: X-ray scattering length $f_{\mathrm{X}}\left[10^{-12} \mathrm{~cm}\right]$ for different atoms. $u$ is the atomic mass, $\mathrm{N}_{e^{-}}$the number of electrons. Adapted from [71].

\begin{tabular}{|l|c|c|c|c|c|c|c|}
\hline Atom & $\mathrm{H}$ & $\mathrm{D}$ & $\mathrm{C}$ & $\mathrm{N}$ & $\mathrm{O}$ & $\mathrm{Na}$ & $\mathrm{Mg}$ \\
\hline \hline$u$ & 1 & 2 & 12 & 14 & 16 & 23 & 24 \\
$N_{e^{-}}$ & 1 & 1 & 6 & 7 & 8 & 16 & 17 \\
$f_{\mathrm{X}}$ & 0.28 & 0.28 & 1.69 & 1.97 & 2.25 & 3.10 & 3.38 \\
\hline
\end{tabular}




\section{\begin{tabular}{l|l} 
Chapter 3 & FUNDAMENTALS
\end{tabular}}

subtraction. For more details about the background subtraction in this thesis refer to Sec. 4.3.4. To compensate for the low contrast of biological samples in solution, high flux photon beams are used in X-ray scattering experiments [72].

\subsection{General Principles of Small-Angle X-Ray Scattering}

In the 1930's Otto Kratky [65] and André Guinier [73] developed the theory as well as the experimental and analytic tools for small-angle X-ray scattering (SAXS). SAXS is a technique used for resolving structural parameters ranging approximately from 1 to $100 \mathrm{~nm}$, such as the size, shape and surface of particle systems or structures [65]. SAXS can be employed on samples in solid, liquid or gaseous phases originating from diverse fields of research, e.g., structural biology and drug discovery. For SAXS photons with an energy $E_{0}$ are elastically scattered inside the sample. The resulting two-dimensional scattering patterns on the detector are azimuthally integrated and the resulting 1D radial intensity profile $I(\boldsymbol{q})$ is plotted against the magnitude of the scattering vector $\boldsymbol{q}\left[\mathrm{nm}^{-1}\right]$

$$
q=\frac{4 \pi}{\lambda} \sin \theta
$$

where $2 \theta$ is the scattering angle and $\lambda$ the wavelength of the incident $X$-rays.

The radial intensity profile $I(\boldsymbol{q})$ is fundamental for SAXS analysis, since it correlates to the incoming electromagnetic wave by the square modulus of the amplitude

$$
I(\boldsymbol{q})=|\psi(\boldsymbol{q})|^{2}
$$

The amplitude of the scattered wave is expressed by

$$
\psi(\boldsymbol{q})=f e^{2 \pi i \boldsymbol{q} r},
$$

where $q$ expresses the momentum transfer difference between the incident and the emitted wave vectors $k_{i n}$ and $\boldsymbol{k}_{\boldsymbol{e} m}$. The atomic scattering factor $f$ is defined as the electron density in the reciprocal space

$$
f=\int_{0}^{\infty} \rho(\boldsymbol{r}) \mathrm{d} r
$$

with $\rho(\boldsymbol{r})$ being the number density of the cloud and $r$ the position of the atom. Each material is composed of electron clouds at the position of each contained 


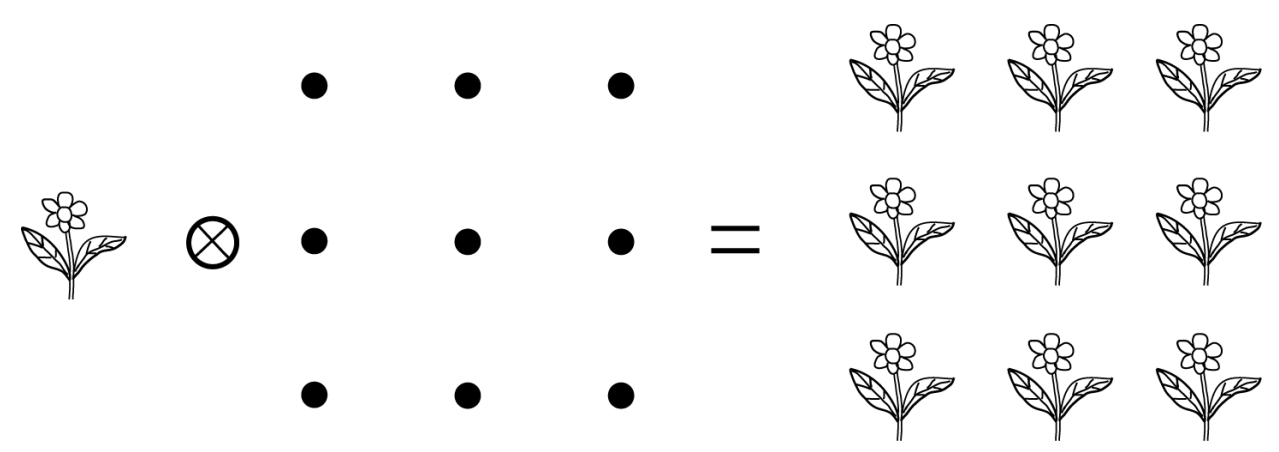

Figure 3.5.: Schematic representation of the form factor (flower) and the structure factor (dots forming the lattice). The convolution yields the crystal structure on the right.

atom. This structure of the unit cell is called the form factor $F(\boldsymbol{q})$. The lattice information corresponding to the position of each atom is described by the structure factor $S(\boldsymbol{q})$. Thus, the scattering signal is the convolution of the structure factor and the form factor (see [74])

$$
I(\boldsymbol{q})=F(\boldsymbol{q}) S(\boldsymbol{q})
$$

In Fig. 3.5 the form factor is represented by the flower and the structure factor by the dots. The convolution of both represents the crystal structure.

\subsubsection{Form Factor}

After processing the detected signal (see Sec. 4.3.4) the shape of the average scatterer is acquired from the radial intensity profile through the form factor $F(\boldsymbol{q})$. The average size of particles is accessible through Guinier's approximation and the structure factor $S(\boldsymbol{q})$ gives information about potential particle interactions. The interference pattern is characteristic of the scatterer. For dilute solutions without inter-particle correlations, the scattering distribution depends only on the particle form factor $F$, which describes the shape of the particles averaged over all orientations. For a particle with (scattering length) density $\rho(\boldsymbol{r})$ it is given by

$$
F(q)=<\int \rho(\boldsymbol{r}) e^{(i \boldsymbol{q} r)} \mathrm{d} V>
$$

where the particle is contained within a volume $V$ and $\langle\ldots\rangle$ denotes orientational averaging. Let $\mathrm{d} V$ be the volume element for a sphere, then $\mathrm{d} V$ is defined as $\mathrm{d} V=r^{2} \sin \theta \mathrm{d} \theta \mathrm{d} \phi \mathrm{d} r$. The form factor of a single sphere with radius $R$ and 


\section{\begin{tabular}{l|l} 
Chapter 3 & FUNDAMENTALS
\end{tabular}}

uniform electron density $\rho$ is then expressed as:

$$
F(\boldsymbol{q})=\rho \int_{0}^{R} \int_{0}^{2 \pi} \int_{0}^{\pi} e^{i \boldsymbol{q} r \cos \theta} r^{2} \sin \theta \mathrm{d} \theta \mathrm{d} \phi \mathrm{d} r
$$

By solving the integrals in Eq. 3.12, for the form factor of a solid and homogeneous sphere follows:

$$
F(\boldsymbol{q})=3 \frac{\sin (\boldsymbol{q} R)-\cos (\boldsymbol{q} R)}{\boldsymbol{q}^{3} R^{3}} .
$$

This form factor for a solid sphere was first was first described by Rayleigh in 1911 [75]. Form factors for different structures, e.g., rods, disks and cylinders, are given or calculated in [76]. Selected form factors are visualized with MATLAB (The MathWorks, Inc., Natick, Massachusetts) and shown in Fig. 3.6.

Figure 3.6 shows $F(\boldsymbol{q})$ for a sphere with a radius $R$ of $10 \mathrm{~nm}$, an infinitely thin disk with a radius $R$ of $10 \mathrm{~nm}$, an infinitely thin rod with the length $L$ of $10 \mathrm{~nm}$, and a cylinder with the length $L$ and radius $R$ of $20 \mathrm{~nm}$ and $10 \mathrm{~nm}$, respectively. For the vimentin-IF tetramers and the ULFs, a cylinder-like shape is assumed, as

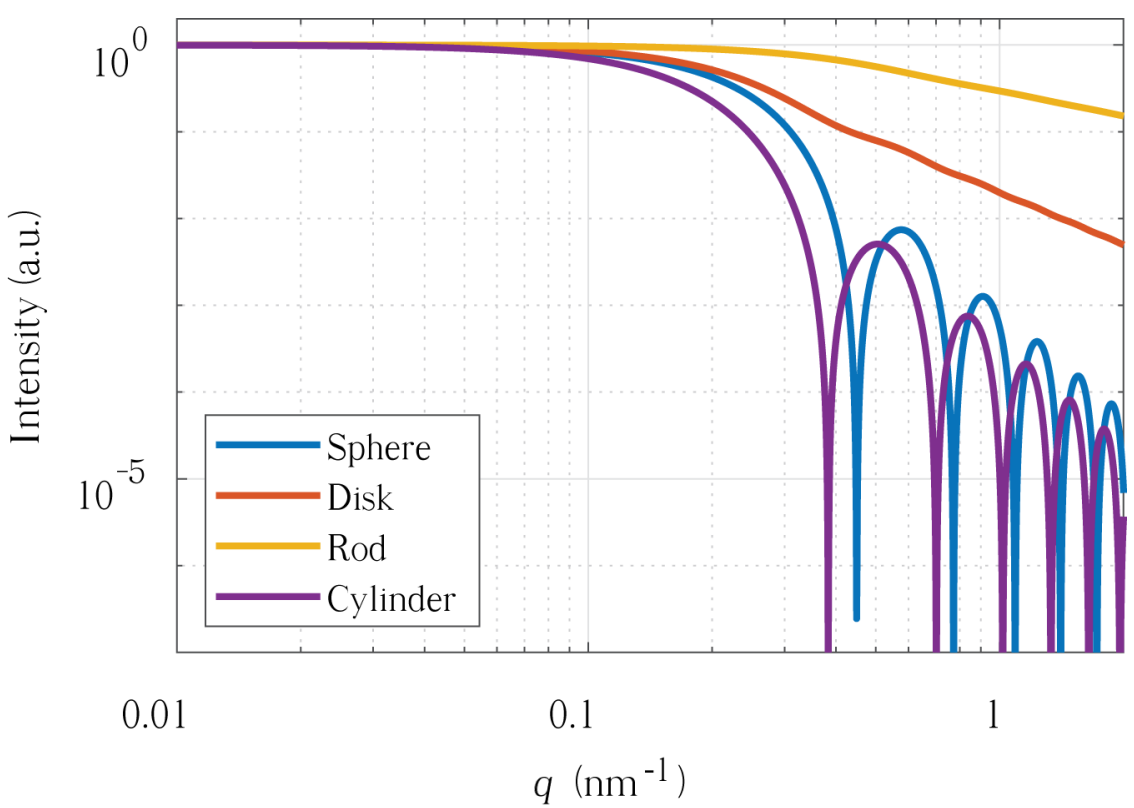

Figure 3.6.: Form factors $F(\boldsymbol{q})$ for a sphere (blue, $R=10 \mathrm{~nm}$ ), an infinitely thin disk (red, $R=10 \mathrm{~nm}$ ), an infinitely thin rod (yellow, $L=10 \mathrm{~nm}$ ) and a cylinder (purple, $R=10 \mathrm{~nm}$, $L=20 \mathrm{~nm}$ ). 
the length and diameter ratios correspond to this (see Fig. 2.1). Hence, the form factor of a cylinders is used during Guinier's analysis for the calculation of the forward scattering intensity $I_{0}$. The form factor for a cylinder is given by [77]

$$
F(\boldsymbol{q})=\int_{0}^{\pi / 2}\left[\frac{2 B_{1}(\boldsymbol{q} R \sin \alpha)}{\boldsymbol{q} R \sin \alpha} \frac{\sin (\boldsymbol{q} L \cos \alpha / 2)}{\boldsymbol{q} L \cos \alpha}\right]^{2} \sin \alpha d \alpha .
$$

with $B_{1}$ as the first order Bessel function.

To obtain the form factor of a single particle variety, the sample needs to be monodisperse and diluted. In the case of polydispersity, the measured form factor contains contributions from each particle variety, resulting in the determination of an average form factor.

\subsubsection{Structure Factor}

In correlated particle systems (non ideal state) interference between scattered photons from neighbouring particles is observed. This is taken into account by the structure factor (see Sec. 3.2). The resulting interference pattern can be used to determine the positions of the scatterers. Figure 3.7 visualizes exemplary scattering patterns for different sample orientations on the detector. Scattering by highly ordered periodic structures, e.g., crystalline structures lead to the formation of

\section{Sample Orientation:}

Random

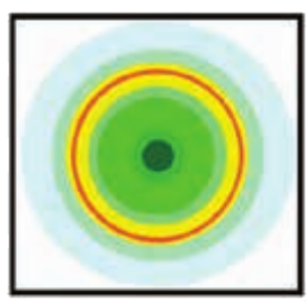

- Dispersions

- Powders
Partial

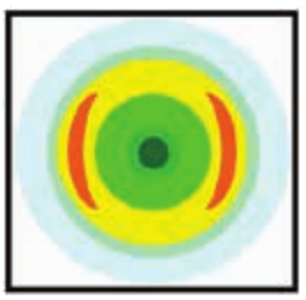

- Fibres

- Sheared Liquids
Complete

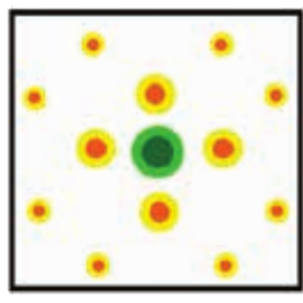

- Single Crystal

Figure 3.7.: Scattering patterns of random, partial and complete ordered samples. Images with permission from [64]. 


\section{\begin{tabular}{l|l} 
Chapter 3 & FUNDAMENTALS
\end{tabular}}

Bragg peaks (see Fig. 3.7, right), given by Bragg's law:

$$
2 d \sin \theta=n \lambda,
$$

where $\theta$ is the incident scattering angle, $d$ the distance between atom layers and $\lambda$ the wavelength of the incident photon.

Partially structures, e.g., fibres or sheared liquids, result in point symmetric scattering patterns, shown in Fig. 3.7, middle. Unordered structure, e.g., dispersions or powders, result in radial symmetric scattering patterns (see Fig. 3.7, left). An example for powder diffraction is silver behenate, used for the sample-detector distance calibrations for inhouse SAXS and scanning-SAXS (see Sec. 4.3.3.1 and Sec. 4.3.3.2) [78].

\subsubsection{Guinier's Approximation, Pair Distance Distribution Function and Radius of Gyration}

Guinier's approximation and the pair distance distribution function (pddf) are two different approaches used to characterize the shape of a particle and is based on its scattering image $[65,73]$. Both approaches are dependent on the forward scattering intensity $I_{0}$. After the incident beam has interacted with the sample, zero angle scattering cannot be distinguished from any unscattered photons, as the unscattered photons transmit the sample with zero angle, too. In addition the incident beam is absorbed, together with the zero angle scattering photons, by a beamstop. Thus, $I_{0}$ is determined via extrapolation from the $1 \mathrm{~d}$ radial intensity profile $I(q)$ with respect to the mass per unit volume $(c)$, molecular weight (MW), the contrast $(\Delta \rho)$, and the partial specific volume $(v)$ of the particle [62]:

$$
I(0)=\frac{c \Delta \rho^{2} v^{2} \mathrm{MW}}{N_{A}}
$$

where $N_{A}$ is Avagadro's number.

The radius of gyration $R_{G}$ provides information about the mass distribution inside all particles and is defined as the mean-squared distance of all scattering volumes from their centre of mass weighted by their scattering densities [62]. Thus, differently shaped objects with the same volume have different $R_{G}$ values.

In 1939 André Guinier showed that by plotting the logarithmic radial intensity $\ln (I(q))$ against $q^{2}$, a so-called 'Guinier plot', $R_{G}$ and $I(0)$ are equivalent to its 
slope and ordinate value and can be obtained by fitting. The linearity of this Guinier plot is valid only for the Guinier region $q R_{G}$ with an upper end defined by structure of the scatterer. For spherical shapes the Guinier region is defined by $q R_{G}<1.3$. Although the Guinier region is smaller for elongated objects, in biological SAXS a Guinier region $q R_{G}<1.3$ is used, based on the weak scattering signal [79]. Relevant relations for this work are known to be [80]:

- Sphere of radius $R$

$$
I(q)=I_{0} e^{\left(-\frac{q^{2} R_{G}^{2}}{3}\right)}
$$

with corresponding radius of gyration of

$$
R_{G}=\sqrt{\frac{3}{5}} R
$$

- Cylinder of radius $R$ and length $L$

$$
I(q)=\frac{I_{0}}{q} e^{\left(-\frac{q^{2} R_{G}^{2}}{2}\right)},
$$

with corresponding radius of gyration

$$
R_{G}=\sqrt{\frac{R^{2}}{2}+\frac{L^{2}}{12}}
$$

Another way for accessing the structural parameters $R_{G}$ and $I_{0}$ is by calculating the pddf $p(r)$. The pddf expresses the distribution of distances between scatterers inside the sample [81]. The pddf uses more data points than the Guinier approximation, but the size of the largest particle $D_{\max }$ and the expression $q<\pi / D_{\max }$ must be known [82]. $R_{G}^{2}$ is given by

$$
R_{G}^{2}=\frac{\int_{0}^{D \max } p(r) r^{2} \mathrm{~d} r}{2 \int_{0}^{D_{\max }} p(r) \mathrm{d} r}
$$

whereas $I_{0}$ corresponds to the area under the pddf [62]

$$
I(0)=4 \pi \int_{0}^{D \max } p(r) \mathrm{d} r .
$$




\section{\begin{tabular}{l|l} 
Chapter 3 & FUNDAMENTALS
\end{tabular}}

\subsection{Microfluidics}

Microfluidics is a versatile tool to investigate biological samples. This chapter will introduce the theoretical framework of microfluidics, which enables us to describe the behaviour of small amounts of liquids $(\approx \mu L)$ in micrometer-sized channels. This section is based on ref. [66].

\subsubsection{Flow at Small Length Scales}

The Navier-Stokes equation (Eq. 3.23), is a non-linear, second order partial differential equation which describes the general motion for an incompressible viscous fluid [66]:

$$
\rho(\boldsymbol{r}, t)\left[\frac{\partial}{\partial t} \boldsymbol{v}+(\boldsymbol{v} \nabla) \boldsymbol{v}\right]=-\nabla p+\eta \nabla^{2} \boldsymbol{v}+\rho g+\rho_{e l} \boldsymbol{E}
$$

where $\rho\left[\mathrm{kg} \mathrm{m}^{-3}\right]$ represents the mass per volume or density, $p$ the pressure $\left[\mathrm{N} / \mathrm{m}^{2}\right]$, the velocity and the dynamic velocity are $v[\mathrm{~m} / \mathrm{s}]$ and $\eta[\mathrm{Pa} \cdot \mathrm{s}]$, respectively. The Navier-Stokes equation compares the inertial forces on the left side of the equation, with the viscous and external forces, namely the pressure gradient $-\nabla p$, the viscous force $\eta \nabla^{2} v$ and body forces $\rho g$ and $\rho_{e l} E$, on the right. The Navier-Stokes equation describes mathematically the beauty and behaviour of hydrodynamic phenomena. The complete solutions of the Navier-Stokes equation have never been found due to the non-linear term $\rho(v \boldsymbol{\nabla}) v$ of the Navier-Stokes equation. Nevertheless, analytical solutions under various boundary conditions can be obtained [66].

The regime of the so-called Stokes flow, with low flow velocities, is in particular relevant for microfluidics and the microfluidic systems developed in this work. Under the assumption that the non-linear term is negligible, we can express all physical variables in the Navier-Stokes equation by characteristic scales, e.g., $v^{*}=$ $\frac{v}{V}$. The ${ }^{*}$ indicates a quantity without physical dimension. Thereby we can analyse the flow in the system. By using dimensionless formulations of $v, \nabla \rho$ and their derivatives, as [66]:

$$
v^{*}=\frac{v}{V}, \frac{\partial}{\partial t^{*}}=\frac{L}{V} \frac{\partial}{\partial t}, \nabla^{*}=L \nabla, p^{*}=\frac{L}{\eta V} p,
$$

and the Navier-Stokes equation for incompressible fluids (Eq. 3.23) excluding 
body forces [66]

$$
\rho\left[\frac{\partial}{\partial t} v+(v \nabla) v\right]=-\nabla \rho+\eta \nabla^{2} v
$$

we obtain:

$$
\rho \frac{V^{2}}{L} \frac{\partial}{\partial t^{*}} \boldsymbol{v}^{*}+\rho \frac{V^{2}}{L}\left(\boldsymbol{v}^{*} \boldsymbol{\nabla}^{*}\right) \boldsymbol{v}^{*}=-\eta \frac{V}{L^{2}} \boldsymbol{\nabla}^{*} p^{*}+\eta \frac{V}{L^{2}} \boldsymbol{\Delta}^{*} \boldsymbol{v}^{*}
$$

This becomes after reduction [66]:

$$
\rho \frac{V L}{\eta}\left[\frac{\partial}{\partial t^{*}} v^{*}+\left(v^{*} \nabla^{*}\right) v^{*}\right]=\nabla^{*} \rho^{*}+\Delta^{*} v^{*}
$$

The dimensionless factor on the left side is the so-called Reynolds number $(R e)$ with $L$ being the characteristic linear dimension and $V$ the characteristic velocity of the system [66].

$$
\operatorname{Re}:=\frac{\rho V L}{\eta}
$$

The Reynolds number compares inertia and viscous forces. For small Reynolds numbers $(R e \ll 1)$ the viscous forces are dominant while for high Reynolds numbers $\left(\operatorname{Re} \gg 10^{3}\right)$ inertia dominate the system [66]. Systems with low Re are considered to be in the "laminar flow regime", where the flow is time reversible and no turbulence occurs. When no external forces occur, thus for systems with low $R e$, the Navier-Stokes equation further simplifies to the Stokes equation [66]:

$$
\nabla p=\eta \nabla^{2} v
$$

For the Stokes equation analytical solutions to the creeping flow problem can be derived in several cases [66].

In this work, aqueous solutions $\left(\rho \approx 10^{3} \mathrm{~kg} / \mathrm{m}^{3}, \eta \approx 10^{-3} \mathrm{~kg} / \mathrm{ms}\right)$ are used inside small channels $L \approx 10^{-4} \mathrm{~m}$ with flow velocities of $V \approx 10^{-3} \mathrm{~m} / \mathrm{s}$. Substituting these values in Eq. 3.27, the Reynolds number is calculated:

$$
\operatorname{Re}_{\text {microfluidics }}=\frac{1 \cdot 10^{3} \mathrm{~kg} / \mathrm{m}^{3} 1 \cdot 10^{-3} \mathrm{~m} / \mathrm{s} 1 \cdot 10^{-4} \mathrm{~m}}{1 \cdot 10^{-3} \mathrm{~kg} /(\mathrm{ms})}=1 \cdot 10^{-1},
$$

thus verifying the assumption of the laminar flow regime for microfluidics. No difference in the velocity vector-field inside the device occurs over time in laminar 


\section{\begin{tabular}{l|l} 
Chapter 3 & FUNDAMENTALS
\end{tabular}}

flow, thus the flow pathlines and streamlines are identical. As each molecule measured over time at one position inside the device shares the identical pathline, the time resolution of the assembly reaction of vimentin corresponds to spatial coordinates inside the device. Combining laminar flow with scanning SAXS, kinetics faster than the exposure time are accessible (see Sec. 5.2.4).

Knowledge of the Reynolds number of a system is of importance in many fields of research and engineering applications, as the flow around an object for similar geometries is the same for identical Reynolds numbers, thus the reduction of size of an object can be compensated by increasing the dynamic viscosity $\eta$ of the system. Applications are models of, e.g., airplanes inside wind channels.

\subsubsection{Diffusive Mixing}

Two different processes of transport occur inside microfluidic devices: Active transport, based on convection, and passive transport, based on diffusion. Similar to the Reynolds number, which compares inertia and viscous drag forces, the Péclet number $(\mathrm{Pe})$ expresses the ratio between convective and diffusive mixing time scales [83].

$$
P e:=\frac{V L}{D}
$$

In other words, the Péclet number is given by comparing the characteristic time for diffusion, thus the time to diffuse a distance equal to its own size, and the shear rate [2].

Characteristic length $L$ and velocity $V$ have been introduced in Sec. 3.3.1. The diffusion coefficient $D\left[\mathrm{~m}^{2} / \mathrm{s}\right]$ is described by using the Einstein relation [66]:

$$
D=k_{B} T \mu
$$

where $k_{B}$ is the Boltzmann's constant and $T$ is the temperature. The mobility of the diffusing particle is described by $\mu$. For spherical particles, e.g., diffusing ions, $\mu$ is defined as:

$$
\mu=1 / 6 \pi \eta r
$$

where $r$ is the radius of the particle and $\eta$ is the dynamic viscosity. Combining Eq. 
3.32 and Eq. 3.31 results in the Stokes-Einstein equation for spherical particles:

$$
D=\frac{k_{B} T}{6 \pi \eta r}
$$

For small Péclet numbers diffusive transport is dominant. For high Péclet numbers the transported particles remain on the flow lines, thus mainly convective transport occurs.

The diffusion coefficient for proteins is on the order of $10^{-11} \mathrm{~m}^{2} / \mathrm{s}$ and for ions around $10^{-9} \mathrm{~m}^{2} / \mathrm{s}[66,84]$. Inserting these values, together with the assumptions taken in Sec. 3.3.1 for microfluidic devices, into Eq. 3.30 yields $P e=10^{4}$ for proteins and $P e=10^{2}$ for ions.

The device acts as a diffusive mixer for vimentin and $\mathrm{KCl}$ ions, thus inducing the assembly of vimentin. Mixing of two separate liquids, one containing proteins and the other containing ions in a microfluidic devices with laminar flow is described in the following. The dominant transport occurring along the laminar flow direction inside the device is convective for both the vimentin and the $\mathrm{KCl}$, as indicated by the Péclet number. The Péclet number indicates which form of transport is dominant and is on the order of $P e_{\mathrm{vim}}=10^{4}$ for vimentin and $P e_{\mathrm{KCl}}=10^{2}$ for $\mathrm{KCl}$ ions. Perpendicular to the flow direction, only diffusive transport occurs as the streamlines inside the device are parallel to each other. The diffusion coefficient for the $\mathrm{KCl}$ ions is two orders of magnitude larger, hence $\mathrm{KCl}$ diffusion is the dominant occurring mixing process inside the device. To fully characterize the mixing process inside the microfluidic devices FEM simulations are utilized in the scope of this work (see Sec. 5.2.1).

\subsection{Cyclic Olefin Copolymer for Microfluidics and Small- Angle X-Ray Scattering}

Cyclic olefin copolymer (COC) are synthesized by chain polymerisation of Norbornene with Ethylene [85]. Figure 3.8 depicts the chemical structure, with $\mathrm{X}$ and $\mathrm{Y}$ being the repeat units. COC forms a class of polymeric material with chemical properties suitable for experiments with biological samples. COC posses high transparency, low water absorption, resistance to acids, alkaline, polar solvents and hydrolysis and is biocompatible [86]. Changing the ratio of the repeat units results in different glass transition temperatures $T_{G}$ [87]. For example, increas- 


\section{\begin{tabular}{l|l} 
Chapter 3 & FUNDAMENTALS
\end{tabular}}

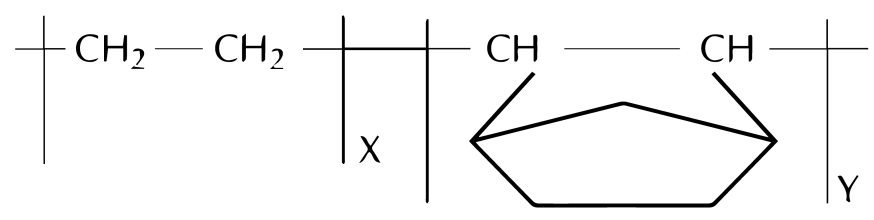

Figure 3.8.: Chemical structure of COC. By changing the ratio between the repeat units $X$ and $Y$ different properties can be adapted. Reproduced from [85].

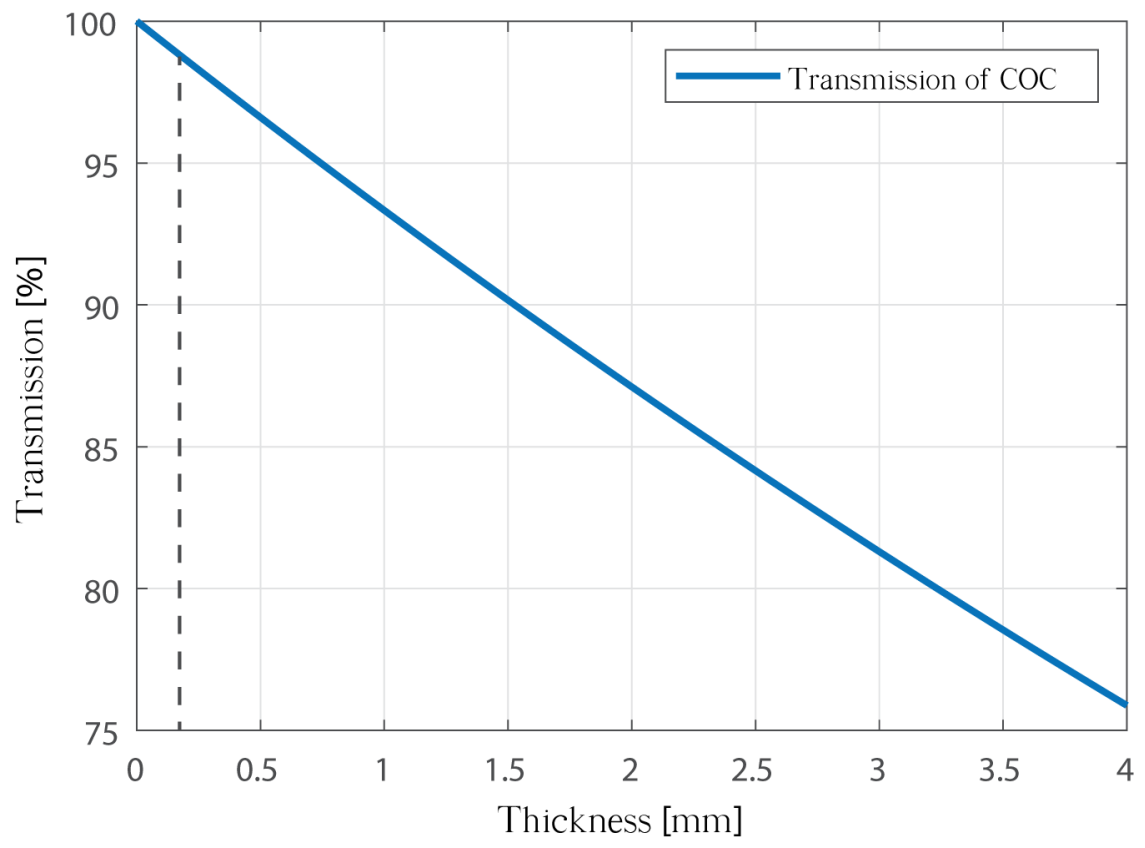

Figure 3.9.: Transmission probability of photons with an energy of $12.5 \mathrm{keV}$ through COC. The dotted line indicates typical thicknesses of microfluidic devices. The attenuation coefficient for COC is calculated with XCOM: Photon Cross Sections Database from the National Institute of Standards and Technology [67] and the transmission consequently calculated using LambertBeer's law (Eq. 3.2). 
ing the Norbonene content in the polymers increases $T_{G}[88,89]$. COC is highly transparent to X-rays in the energy regime employed for SAXS. The linear attenuation coefficient for COC at $12.5 \mathrm{keV}$ is calculated with XCOM: Photon Cross Sections Database from the National Institute of Standards and Technology [67] and Lambert-Beer's law (Eq. 3.2). The transmission probability of photons depends on the material thickness is shown in Fig. 3.9.

Typical thicknesses of microfluidic devices are on the order of $10^{3} \mu \mathrm{m}$. Compared to polydimethylsiloxane (PDMS) the attenuation of COC is 7 times less, rendering it, combined with the material properties of COC, suitable for combining SAXS and microfluidics [90].

\subsection{Visible Light Fluorescence Microscopy}

\subsubsection{Basic Principles of Fluorescence}

Fluorescence describes the absorption and emission of characteristic photons by electrons during transitions of different energy states. If the energy of the incident photon is larger than the binding energy of an electron inside a fluorophore, the electron will absorb the photon, resulting in the electron transitioning from the ground state (S0) to an excited state (S1). The absorption process occurs on the order of femtoseconds [61], resulting in the fluorophore being unable to undergo conformational changes (Franck-Condon principle [91]). The absorption spectrum results of three main processes:

- Electronic

Wavelengths $\lambda$ of the incident photons in the ultraviolet to visible light regime (200 $\mathrm{nm}<\lambda<700 \mathrm{~nm}$ ) are absorbed in the electronic gap transition between different states (Sn). This results in the electron to transition to an excited state.

- Vibrational

Wavelengths $\lambda$ of the incident photons in the infrared regime (700 $\mathrm{nm}<\lambda$ $<10^{3} \mathrm{~nm}$ ) are required to change between different vibrational levels inside one state Sn.

- Rotational

Wavelengths $\lambda$ of the incident photons in the microwave regime $\left(10^{-3} \mathrm{~m}<\lambda\right.$ 


\section{\begin{tabular}{l|l} 
Chapter 3 & FUNDAMENTALS
\end{tabular}}

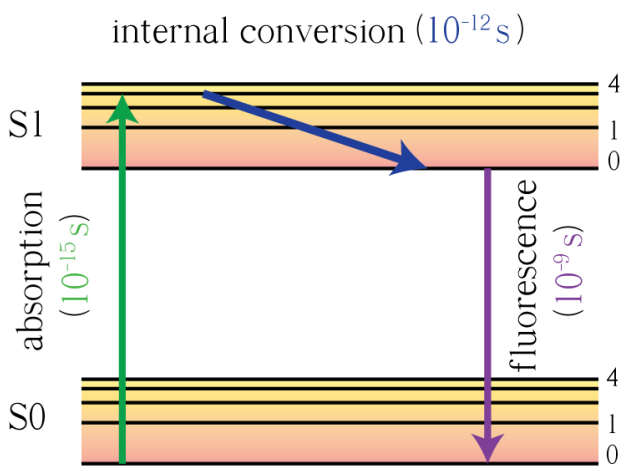

Figure 3.10.: Jablonski diagram visualising possible pathway ways for an exited molecule to return from the exited state S1 to the ground state S0. After absorption of an incident photon the electron transits to from S0 to S1 (green arrow). Internal conversion occurs, reducing the vibrational level of the electron (blue arrow). Subsequently fluorescence occurs (purple arrow) with a characteristic wavelength, while the electron returns to the ground state.

$<1 \mathrm{~m}$ ) induce changes between different rotational states in each vibrational state.

One of the first people to describe the excitation and de-excitation of a molecule was A. Jabloński in 1933 [92]. Possible pathways between different excitation states are indicated by the Jabloński diagram, shown in Fig. 3.10. To excite an electron from S0 to S1, it must absorb an energy greater than its binding energy, typically via an incident photon (see Fig. 3.10). In the exited state, internal conversion (see the blue arrow in Fig. 3.10) occurs on order of $10^{-12} \mathrm{~s}$. As fluorescence lifetimes are longer (purple arrow in Fig. 3.10, $\approx 10^{-9} \mathrm{~s}$ ) the fluorescence mainly occurs from the lower vibrational level of the excited state. Fluorescence radiation usually has a longer wavelength $\lambda$ than the incident photon. This loss of energy is based on the internal conversion and is named Stokes shift, after G.G. Stokes, who first described this phenomenon in 1852 [93]. The Stokes shift is fundamental for fluorescence microscopy as it enables the differentiation between excited and emitted light [94]. 


\subsubsection{Epifluorescence Microscopy}

An epifluorescence microscope is an application of the Stokes shift and is shown schematically in Fig. 3.11. Light is produced by a light source and filtered by an excitation filter to consists only of photons with the desired excitation wavelength. The transmitted light is reflected by the dichroic mirror and focused by the objective on the sample. Here, the fluorophores contained inside the sample are excited by the incident light and emit fluorescence light. The objective collects the emitted fluorescence light and transfers it back onto the dichroic mirror, where the fluorescence light is transmitted through the dichroic mirror, based of the different wavelength of the fluorescence light originating from the Stokes shift. After passing through the emission filter, the fluorescence signal is recorded by a camera.

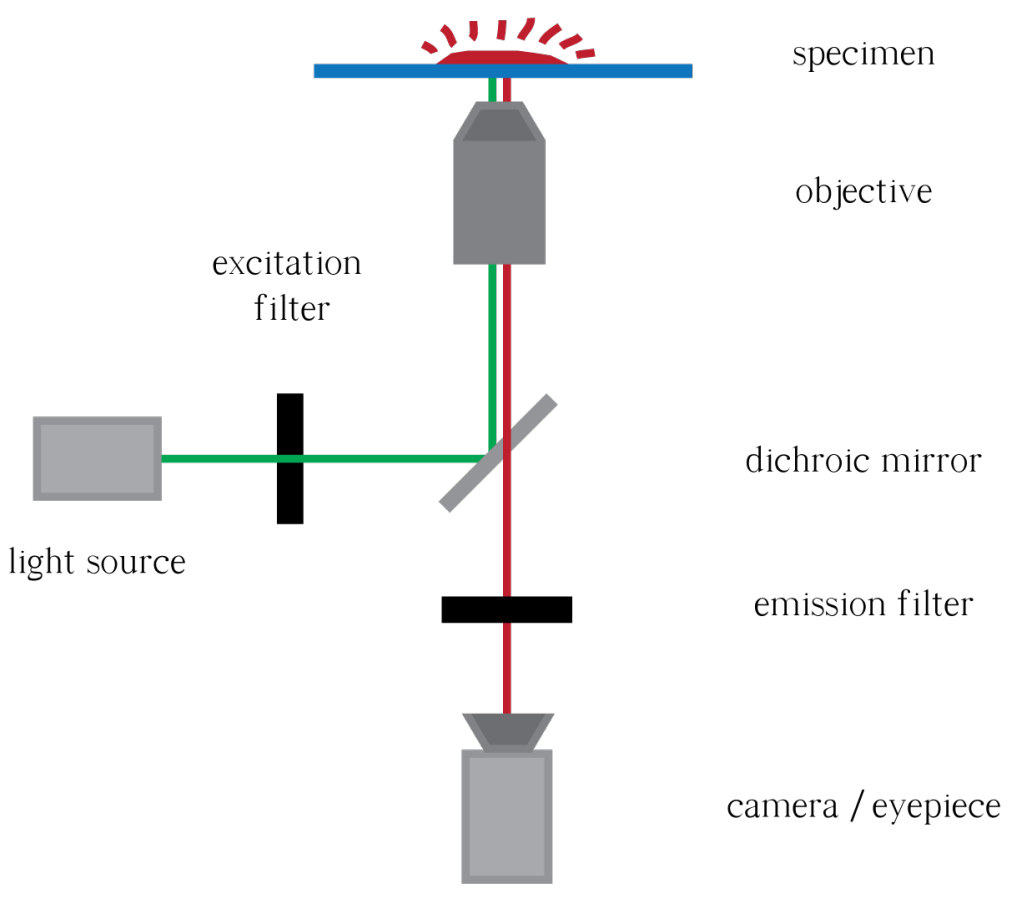

Figure 3.11.: Schematic layout of an epifluorescence microscope. Excitation light (green) is produced by a light source and filtered to contain only photons with the excitation wavelength. The light is reflected and focused on the specimen by the dichroic mirror and the objective, respectively. Emitted fluorescent light of the specimen is collected by the objective and transmitted through the dichroic mirror and an emission filter onto the camera or eyepiece. 


\section{\begin{tabular}{l|l} 
Chapter 3 & FUNDAMENTALS
\end{tabular}}

\subsection{Finite Element Method}

Partial differential equations (PDE) are commonly employed to describe solutions to occurring problems in physics. Although the description of the solution by the PDE is exact, often no analytical solution can be determined. One example for a PDE, which can only be solved in special cases by applying boundary conditions, is the Navier-Stokes equation (Eq. 3.23). The finite element method (FEM) is a numerical method to circumvent the analytical solution by approximating the PDE with numerical model equations, which can be solved. In turn the solutions of the numerical model equations approximate the "real" solution from the original PDE.

One simple example of the finite element method is shown in Fig. 3.12. The function $f(x)$ is unknown. The surface under $f(x)$ is estimated by different known surfaces $V_{*}$. The accuracy of the result given by this calculation is dependent on the step size, thus the amount of surfaces $V_{*}$ used to estimate the original function $f(x)$. In this work FEM simulations are employed during the development of microfluidic devices, to resolve the flow profiles and mixing properties, thus enabling the geometry to be adapted to the experimental needs prior to manufacturing. By varying the FEM element size in dependence of the occurring dynamic, the obtained solution is improved, while coarser mesh elements in less dynamic

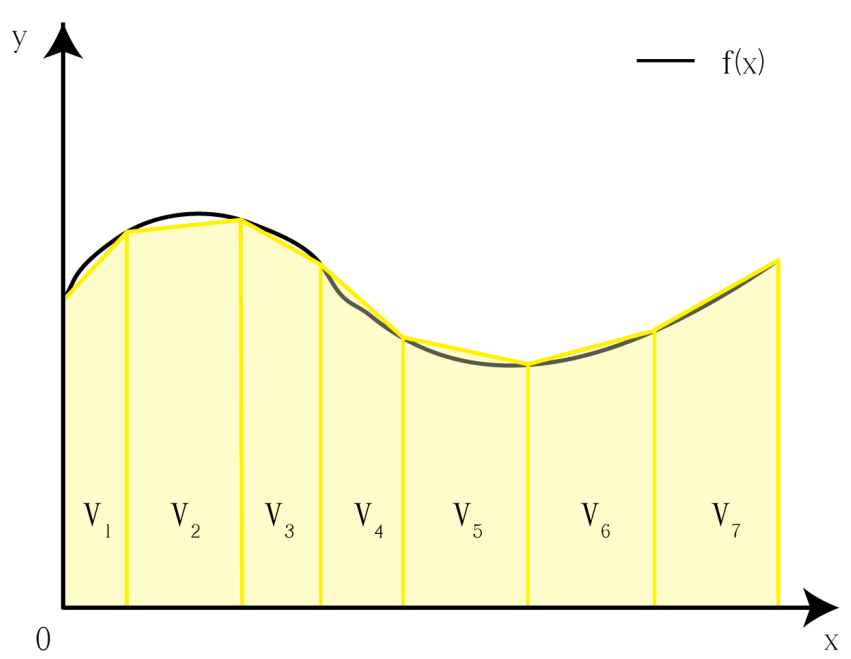

Figure 3.12.: Functional principle of the finite element method. The surface under the unknown function $f(x)$ is estimated by combining known surfaces $V_{*}$. Increasing the number of surfaces increases the accuracy, but also the computation time. 
regions compensate for higher computation times of dynamic regions. The results are shown in Sec. 5.2.1. 



\section{Experimental Methods}

This chapter starts with an introduction to sample preparation, followed by the manufacturing process of the COC microfluidic step devices, hereinafter referred to as 'device'. Subsequently, the device holder is introduced. Finally, the different experimental setups and methods used are introduced, as well as the respective data acquisition procedures.

\subsection{Sample Preparation}

\subsubsection{Vimentin Reconstituition}

Escherichia coli bacteria are used to express human vimentin wild type protein in good purity and yield after purification. Vimentin purification is conducted by Susanne Bauch (Institut für Röntgenphysik, Göttingen). The protocol used is based on [4] and described in detail in [95]. In short, escherichia coli bacteria are transformed to express human vimentin wild type protein. The plasmid is provided by Harald Herrmann (Universitätsklinikum Erlangen, Erlangen, Germany). Several steps of purification, including centrifugation, homogenization, washing steps and two ion exchange chromatography steps, result in vimentin concentrations of up 
to $3-4 \mathrm{mg} / \mathrm{ml}$. The vimentin quality is monitored by electrophoresis after each purification step [95]. Aliquots of the denatured and purified vimentin are stored at $-80{ }^{\circ} \mathrm{C}$.

Different types of assembly experiments with human vimentin wild type protein are performed in this work. For static SAXS and time-resolved fluorescent imaging, $5 \mathrm{mM}$ tris(hydroxymethyl)aminomethan (TRIS, Carl-Roth $\mathrm{GmbH}$, Karlsruhe, Germany) buffer is used, whereas scanning SAXS experiments are performed in $2 \mathrm{mM}$ phosphate buffer (Carl-Roth $\mathrm{GmbH}$, Karlsruhe, Germany). Prior to all experiments, the vimentin is reconstituted by step-wise dialysis of the vimentin against a suitable urea-free buffer system. The thawed vimentin in the storage solution with a monomer weight of $54 \mathrm{kDa}$ is filled in $50 \mathrm{kDa}$ cut off dialysis tubing (SpectraPor, Carl Roth $\mathrm{GmbH}$, Karlsruhe, Germany) and placed at room temperature in the respective buffer system for the experiment containing $6 \mathrm{M}$ urea. The buffer is diluted with urea-free buffer every 30 minutes, reducing the urea concentration in a step-wise manner to $4 \mathrm{M}, 2 \mathrm{M}, 1 \mathrm{M}$ and $0 \mathrm{M}$. The dialysis is continued overnight in fresh $0 \mathrm{M}$ buffer at $8{ }^{\circ} \mathrm{C}$. After the dialysis, the vimentin concentration is measured by ultraviolet-visible (UV) spectroscopy (Nanodrop, Thermo Scientific Technologies, Wilmington, USA). The dialyzed vimentin is stored at $4{ }^{\circ} \mathrm{C}$ for up to five days before being discarded.

\subsubsection{Vimentin Labeling}

Fluorescent imaging of the vimentin assembly requires the attachment of a fluorophore to the vimentin. Labeling is performed using the protocol from Anna Schepers (Institut für Röntgenphysik, Göttingen), based on Ref. [20].

Briefly, vimentin in $50 \mathrm{kDa}$ cut off dialysis tubing is dialyzed against labelling buffer (5 M urea, $50 \mathrm{mM}$ phosphate buffer, pH 7.0). $10 \mathrm{mM}$ Atto647N (ATTO-TEC $\mathrm{GmbH}$, Siegen, Germany) is dissolved in water-free dimethyl-sulphoxide (DMSO, Carl Roth $\mathrm{GmbH}$, Karlsruhe, Germany) and $20 \mu \mathrm{L}$ are added in a steps of $5 \mu \mathrm{L}$ to $1 \mathrm{~mL}$ of vimentin $(1 \mathrm{mg} / \mathrm{ml})$. The mixture is kept at room temperature for 2 hours, before $100 \mu \mathrm{L}$ of $1 \mathrm{M}$ cysteine (Carl Roth $\mathrm{GmbH}$, Karlsruhe, Germany) is added to remove the excess free dye. The labelled vimentin is separated from the excess dye by size exclusion chromatography in a $300 \mathrm{~mm}$ Bio-Gel P-30 (Bio-Rad Laboratories $\mathrm{GmbH}$, Feldkirchen, Germany) polyacrylamide gel column. Fractions are collected in aliquots and the vimentin and dye concentration is measured by UV spectroscopy. The highest fractions are pooled and dialyzed in $50 \mathrm{kDa}$ cut 
off tubing against the storage buffer $(8 \mathrm{M}$ urea in $2 \mathrm{mM} \mathrm{PB}, \mathrm{pH}=7.5)$ overnight at $4{ }^{\circ} \mathrm{C}$. The labelling ratio of the vimentin is calculated the next day and the labelled vimentin is stored in aliquots at $-80{ }^{\circ} \mathrm{C}$.

\subsubsection{Vimentin Assembly}

In this work, vimentin assembly is investigated with three different methods. For static SAXS and fluorescent imaging vimentin is kick-start assembled in $5 \mathrm{mM}$ TRIS buffer ( $\mathrm{pH} \mathrm{7.5).} \mathrm{To} \mathrm{do} \mathrm{so,} \mathrm{the} \mathrm{vimentin} \mathrm{is} \mathrm{step-wise} \mathrm{dialyzed} \mathrm{into} 5 \mathrm{mM}$ TRIS buffer (see Sec. 4.1.1) and then mixed 1:1 with kick-start solutions. These solutions are identical for static SAXS and fluorescent imaging and contain $5 \mathrm{mM}$ TRIS, $200 \mathrm{mM} \mathrm{KCl}$ and either $0 \mathrm{mM}, 10 \mathrm{mM}, 40 \mathrm{mM}$ and $100 \mathrm{mM}$ of ACC (SigmaAldrich, St. Louis, Missouri, USA) or PBA (Sigma-Aldrich, St. Louis, Missouri, USA).

For fluorescent imaging, 3-5 \% labelled vimentin (see Sec. 4.1.2) is added before the step-wise dialysis. The dialyzed vimentin is diluted with dialysis buffer to a concentration of $0.4 \mathrm{mg} / \mathrm{ml}$ prior to the kick-start assembly. The mixture is assembled for different time points inside reaction tubes at $37^{\circ} \mathrm{C}$ before being imaged at room temperature (see Sec. 4.4 for details).

In the static SAXS experiments the dialyzed vimentin is mixed with the respective kick-start solution and is directly loaded inside the quartz glass capillary (Hilgenberg, Malsfeld, Germany). The capillary is sealed with vacuum wax (Hampton Research, Aliso Viejo, USA) and the ensemble is placed in a water bath at $37^{\circ} \mathrm{C}$ for 4 hours (see Sec. 4.3.3.1).

Scanning SAXS experiments are performed in $2 \mathrm{mM}$ phosphate buffer. To do so, the vimentin is dialyzed in a step-wise manner from the storage buffer into $2 \mathrm{mM}$ phosphate buffer. The vimentin is drawn inside a glass syringe (Hamilton Gastight glass syringes, Bonaduz, Switzerland) and assembled inflow inside the microfluidic COC devices (see Sec. 4.3.3.2). The manufacturing of the microfluidic COC devices is explained in the following. 


\subsection{Cyclic Olefin Copolymer Microfluidic Step Devices}

Cyclic olefin copolymer (COC) is a suitable material for X-ray compatible microfluidic devices and has previously been used for structure determination of crystallized biomolecules [96]. One focus of this work is to further develop COC microfluidic devices for vimentin assembly, presented in Sec. 5.1 [97]. Interactions from the sample and inner surface of the device, i.e., the microfluidic channel walls, are evaded by engulfing the sample inlet with buffer solution, resulting in hydrodynamically focusing the sample in the centre of the channel. The geometry of the mixing region is sketched in Fig. 4.1. In the inset the difference in height between the step channel and the mixing region is visible, which is shown for a manufactured COC device in Fig. 4.3f. Note that sheath inlets are employed to increase the time resolution of the reaction, as suggested by Park et al. [98].

Although manufacturing of a X-ray compatible microfluidic step device has been shown for microfluidic devices using a combination of ultra-violet-lightcurable glue (NOA, Norland Optical Adhesives, Cranbury, NJ, USA) and Kapton [60], fundamental differences in the processing between COC and NOA-Kapton required a different approach for the manufacturing process of COC step devices. Compared to NOA-Kapton devices, COC step devices are made solely of a single material. To do so, the bonding of different parts of the device is performed by

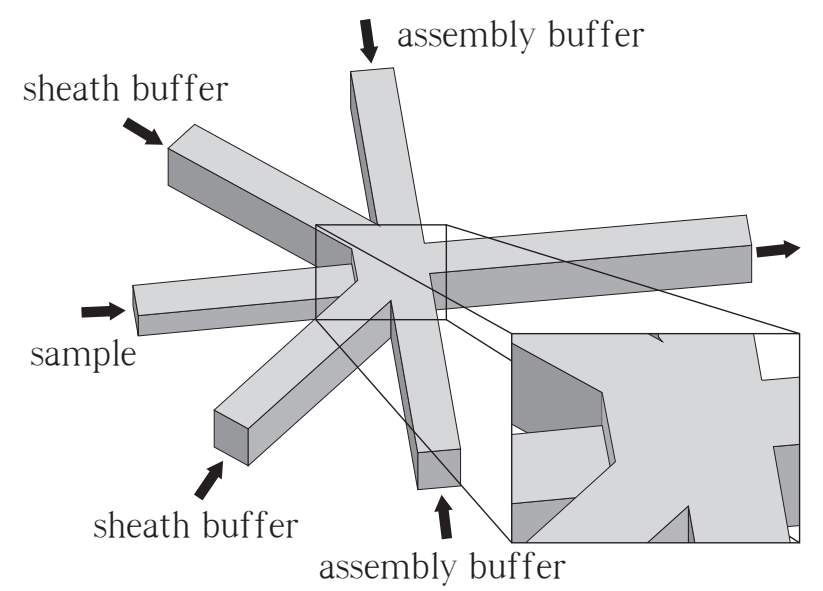

Figure 4.1.: Schematic of the mixing region of a microfluidic step device. The sample in the central channel is hydrodynamically focused to the centre by the inflow from the sheath and assembly buffer channels. The inset shows the step of the sample channel, visible by the difference in height. 
hot embossing instead of using adhesives. As two halves need to be combined to assemble a microfluidic step device, both halves must be entirely heated above the glass transition temperature $T_{G}$ of the employed $C O C$, before the connecting surfaces can be combined by applying external force. Short bursts of heat, similar to those used for non-step COC devices (see Sec. 5.1 [97]), do not work. As the whole device is above $T_{G}$, applying external force deforms the structure of the channels. Therefore, a different approach of combining the two halves was developed and explained in the following.

\subsubsection{Device Manufacturing}

FEM simulations are performed to find the appropriate device geometry, e.g., step height and channel widths. Further details of the FEM simulations are described in Sec. 5.2.1. The geometry is then milled inside brass with a resolution of $10 \mu \mathrm{m}$. This brass mould is used to manufacture all devices. A schematic representation of hot embossing steps is shown in Fig. 4.2, whereas selected manufacturing steps are shown in Fig. 4.3. Two different types of COCs are used in the manufacturing of the devices. COC 8007 with a thickness of $20 \mu \mathrm{m}$ (TOPAS Advanced Polymers $\mathrm{GmbH}$, Raunheim, Germany) and a $T_{G}$ of $78{ }^{\circ} \mathrm{C}$ [99] and COC 6013 with thicknesses of $125 \mu \mathrm{m}$ and $175 \mu \mathrm{m}$ (mcs-foil-080 and mcs-foil-081, Microfluidic ChipShop GmbH, Jena, Germany) and a $T_{G}$ of $142{ }^{\circ} \mathrm{C}[100]$.

The brass mould (see Fig. 4.2a) is cleaned with 2-propanol and three different layers of COC are arranged on top. Directly on the brass mould is one layer of COC 8007 with a thickness of $20 \mu \mathrm{m}$. Two layers of COC 6013 with a thickness of $125 \mu \mathrm{m}$ and $175 \mu \mathrm{m}$ are subsequently added (see Fig. 4.2b), followed by a glass slide (75 mm x $50 \mathrm{~mm} \times 1 \mathrm{~mm}$, Corning Inc., Corning, USA). The entire ensemble is then wrapped in aluminium foil and placed in a hot press $(\mathrm{PW} 10 \mathrm{H}, \mathrm{P} / \mathrm{O} /$ Weber $\mathrm{GmbH}$, Remshalden, Germany), preheated at $170{ }^{\circ} \mathrm{C}$ for 5 minutes. $10 \mathrm{kN}$ of force are applied for $2 \times 2$ minutes before the hot press is cooled with water to $55^{\circ} \mathrm{C}$. Remaining force is released and the brass mould is extracted (see Fig. 4.3a). The combined COC layers (see Fig. 4.2c) are lifted from the brass mould (Fig. 4.3b) and the supernatant COC is cut using a scalpel. Holes are punched in every second half at the planned outlets using a biopsy puncher (Harris Uni-CoreTM puncher, Plano, Wetzlar, Germany), shown in Fig. 4.3c. Both types of halves, with and without punched holes, are aligned on top of each other and fixed using tape. Alignment is facilitated using cone structures, located in the corners of each 


\section{\begin{tabular}{l|l} 
Chapter 4 & EXPERIMENTAL METHODS
\end{tabular}}

a)
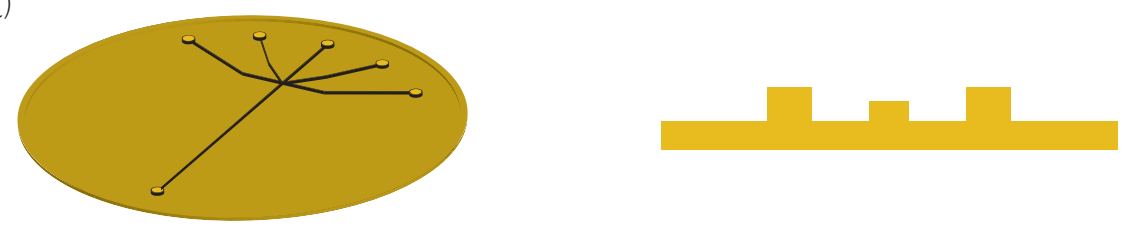

b)
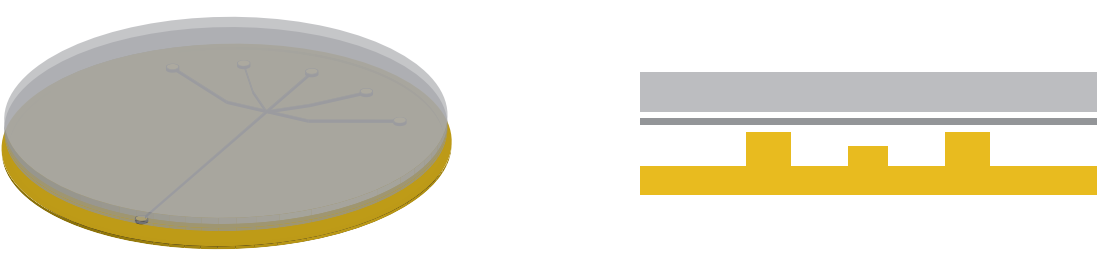

c)
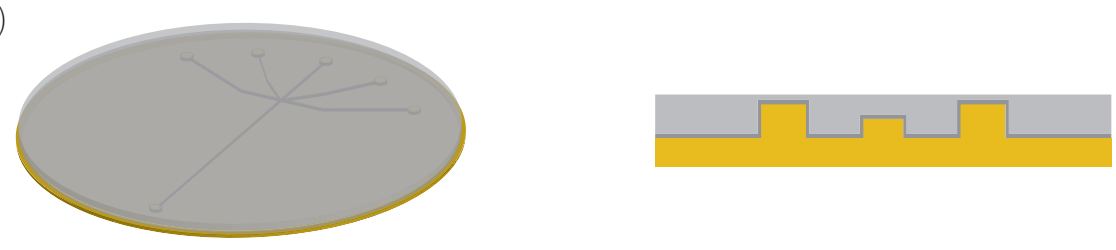

d)
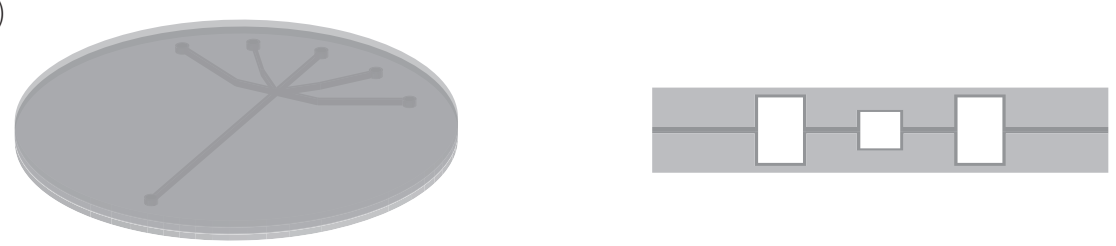

Figure 4.2.: Hot embossing steps for COC step devices. From top to bottom: a) The brass mould is cleaned and b) three layers of COC are arranged on top. c) After hot embossing the layers are combined and the process is repeated for a second half. d) In the last shown step both halves are aligned and hot embossed at temperature below the $T_{G}$ of the COC 6013 , but above the $T_{G}$ of the $\operatorname{COC} 8007$. 

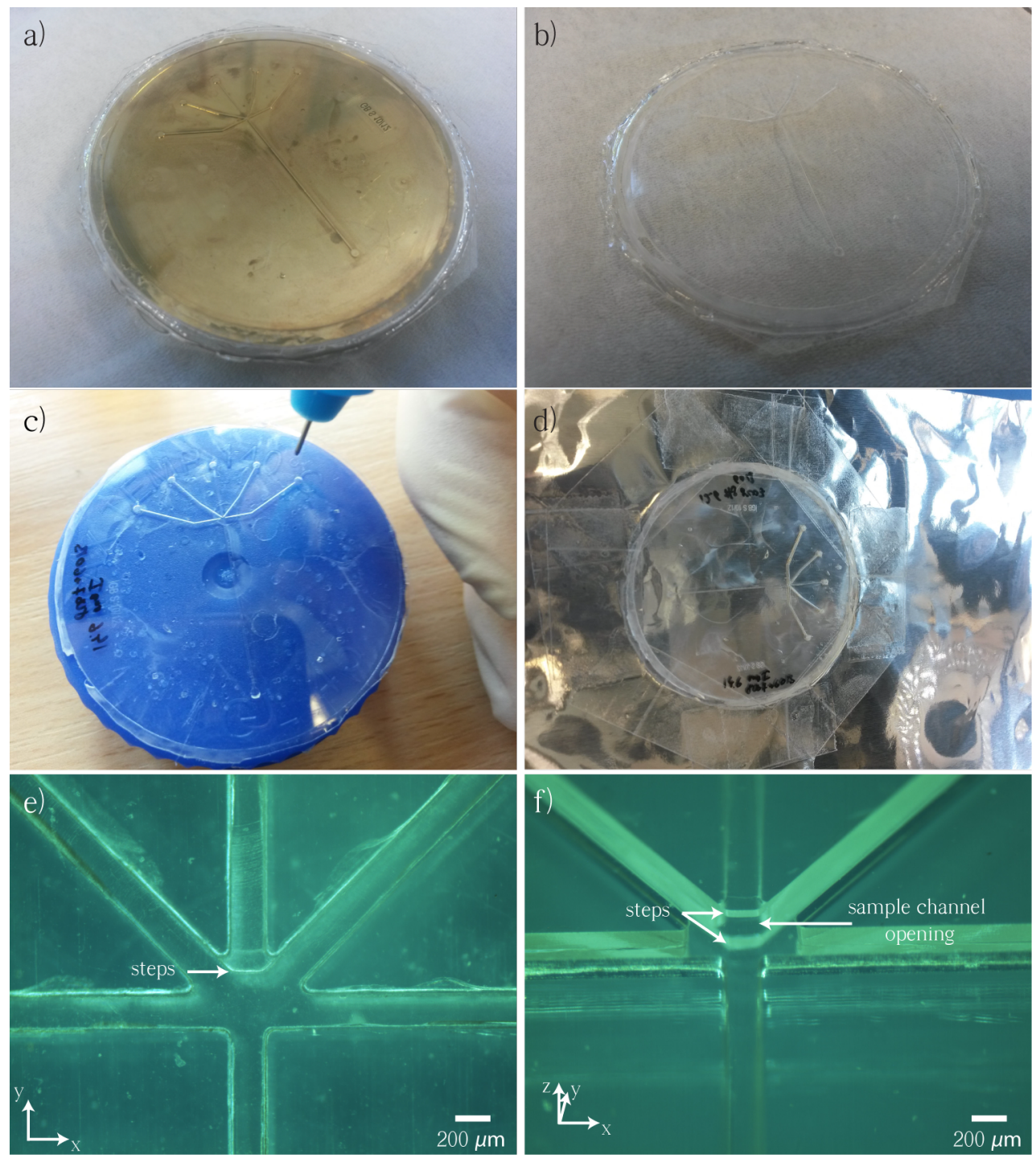

Figure 4.3.: Selected steps of $\mathrm{COC}$ device manufacturing. a) The merged $\mathrm{COC}$ layers are b) removed from the brass mould. c) Holes for tubing connection are punched and d) two halves aligned for merging in a second hot press step. e) and f) shows two halves aligned and combined to a finished device. 
half, which act as a key-lock system. The aligned halves are again wrapped in aluminium foil and placed in the preheated hot press at $110{ }^{\circ} \mathrm{C}$ for 5 minutes. $10 \mathrm{kN}$ of force is subsequently applied for $2 \times 2$ minutes before cooling the hot press with water to $55{ }^{\circ} \mathrm{C}$. The finished COC device is extracted (see Fig. 4.2d) and imaged with a stereo microscope. Exemplary images of a finished device are shown in Fig. 4.3e+f. The central channel for the sample inflow is in the upper middle. The step is indicated by arrows and visible in the central region.

\subsubsection{Establishing Flow Connections with Microfluidic Devices}

After completing the assembly of the two halves, the microfluidic device needs to be connected to the tubing system. The connection should fulfil different specifications to allow for reliable operation during data collection. The specifications identified are described in the following.

Leak Tight Connection

Microfluidic devices operate at flowrates of a few $\mu \mathrm{L} / \mathrm{h}$. Consequently, leakages of small amounts have a great impact on the resulting flow inside the microfluidic channels. As a result, the agreement of the whole dynamics of the sample system with the FEM simulations are dependent on reliable connections.

Transportability of Connected Devices

Time-resolved scanning SAXS experiments in this work are performed during beamtimes at synchrotrons. During data acquisition no direct interaction with the sample system is possible due to the ionizing radiation. The ability to preconnect and transport a device directly increases the measuring time, as fewer tasks have to be fulfilled once the sample is changed.

Reproducible Establishment of Flow Connections

The connection should further be established in a consistent way. As there are six device-tubing connections needed for performing the experiment (sample, $2 x$ sheath buffer, $2 x$ assembly buffer and one outlet) they should all reliably connect so that time is not spent adjusting individual connections.

Mechanical Stability

Inherent to scanning SAXS is the constant movement of the sample while measuring. Mechanical stability is needed to conserve the connections and to obtain measurements on the same positions for background subtraction. 


\subsubsection{Functional Principle of the Device Holder}

To address these specifications the device holder designed in Sec. 5.1 [97], based on [101], is further adapted. The first generation sample holder, presented Sec. 5.1 greatly improved the workflow of manually connecting the device to the microfluidic pumps during data acquisition and met the requirements stated in Sec. 4.2.2. A next generation, improving on mechanical stability and the establishment of flow connections was developed in the scope of this thesis. The mechanical stability of the device holder is increased by adjusting material thickness on selected parts and introducing support structures. Increasing the number of holding screws reduced the torque induced by the holding screws during the assembly process. Detaching of the employed polyethylene (PE) tubing (inner diameter $0.38 \mathrm{~mm}$ and outer diameter $1.09 \mathrm{~mm}$, Intramedic Clay Adams Brand, Becton Dickinson and Company, Sparks, USA) during the tightening of the holding screws was prevented by shearing and subsequent fixation of the upper aluminium plate against the middle aluminium plate prior to the addition of the device (see Fig. $4.5 \mathrm{~d}$ ), thus holding the PE-tubing in place by gently squeezing it.

The device holder consists of three aluminium plates that share a cutout for the centre region of the microfluidic device and is displayed in Fig. 4.4. Leak-tight connections between the channel openings of the microfluidic COC device and the outlet of the PE-tubing are established by deformation of O-rings ( $1 \mathrm{~mm}$ in inner diameter and $1 \mathrm{~mm}$ cross section). The deformation is induced by screwing
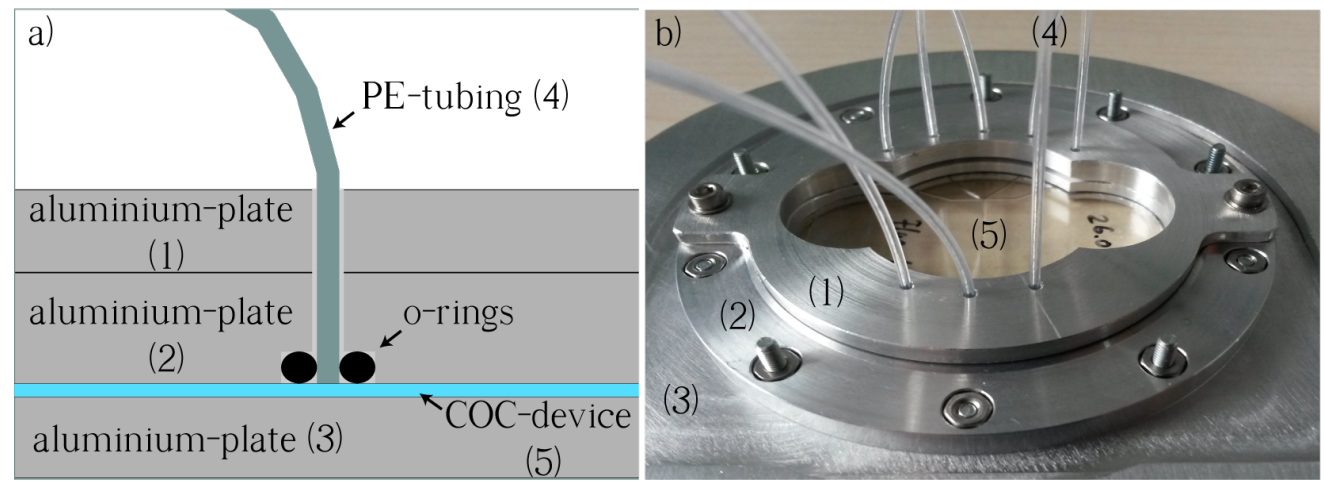

Figure 4.4.: Architecture and functional principle of the device holder. The cross section is shown in a) and the assembled device holder in b). Leak-tight connection between the COCdevice (5) and the PE-tubing (4) is obtained by deforming the O-rings between the middle (2) and lower aluminium plate (3). Shearing the upper (1) against the middle aluminium plate (2) fixates the PE-tubing. All aluminium plates share an opening corresponding to the mixing region of the microfluidic device b) (5). 


\section{\begin{tabular}{l|l} 
Chapter 4 & EXPERIMENTAL METHODS
\end{tabular}}

the aluminium plates together, which subsequently wedges the O-rings into an indentation. This holds in addition the device in place. The device holder contains various screw holes on the lower aluminium plate suitable for connection to the sample stage at the beamline (not shown).

\subsubsection{Device Holder Assembly}

All device holder parts and the O-rings are cleaned with 2-propanol prior to assembly. Main steps of the device holder assembly are shown in Fig. 4.5. PE-tubing is threaded trough the upper aluminium plate (see Fig. 4.4) and subsequently trough the middle aluminium plate (see Fig. 4.5a). To facilitate threading, each PE-tubing end is wedged using a scalpel. The gap between both plates is closed and O-rings are applied to each PE-tubing. The cone-shaped indentations for the O-rings are visible at the intersection of the PE-tubing and the (middle) aluminium plate (see Fig. 4.5b). PE-tubing ends are cut straight before being drawn through both aluminium plates. The tubing is pulled back through the plates until it is level with the O-rings (see Fig. 4.5c). By gently rotating the upper aluminiumplate against the middle aluminium plate and fixing this position using holding screws, the PE-tubing is locked in place (see Fig. 4.5d). The COC-device is aligned with the channel openings facing the O-rings and temporarily held in position using conventional tape (see Fig. 4.5e). The tape is applied away from the O-rings to prevent uneven force distributions in the subsequent steps. The lower aluminium plate is attached with screws. Equally tightening the screws in pairs opposite to each other in small steps assures uniform clamping force on the O-rings.

A glass syringe is drawn up with $2 \mathrm{mM}$ degassed and filtered phosphate buffer, and a hypodermic-needle (B.Braun Melsungen AG, Melsungen, Germany) is connected to the open end of the outlet PE-tubing. The microfluidic device is gently filled with buffer solution and the leak-tight connection between the device and the device holder is verified via visual inspection (see Fig. 4.5f). When no air bubbles remain in both the device and the tubing, the syringe is removed, and a lighter is used seal the tubing ends by melting the PE-tubing. The ensemble is now ready for the integration into the sample stage of the beamline and subsequent measurements. The employed SAXS setups with the corresponding data acquisition steps are described in the following. 

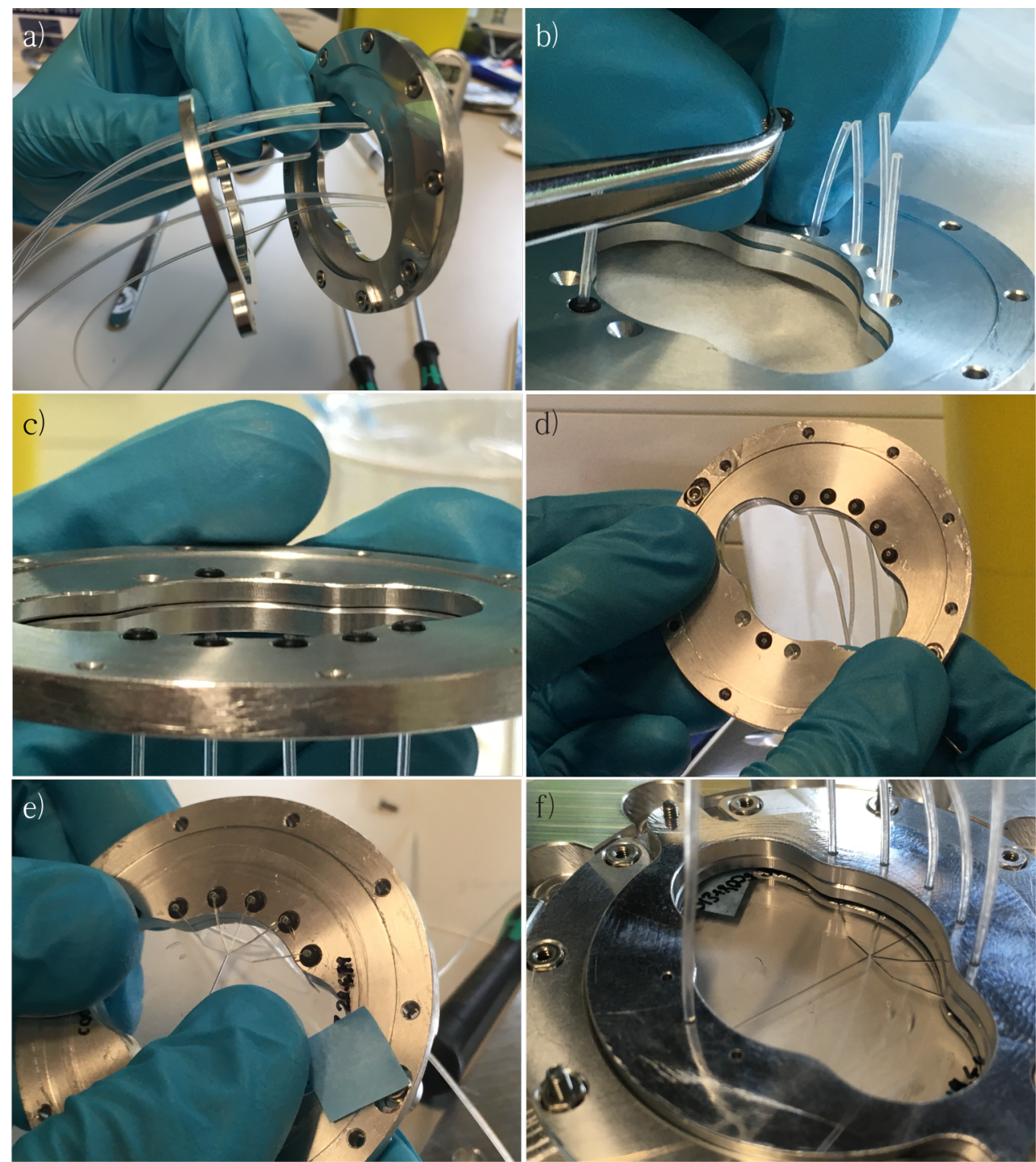

Figure 4.5.: Steps of the device holder assembly. a) PE-tubing is threaded through the upper and middle aluminium-plate. b) O-rings are applied to the end of each PE-tubing and c) levelled with the middle aluminium plate. d) Rotating both plates in respect to each other holds the PEtubing in position. e) The microfluidic COC device is applied and f) after screwing the ensemble to the lower aluminium plate the connections are tested. 


\subsection{Small-Angle X-Ray Scattering}

SAXS is one of the main methods employed in this work, and is introduced in Sec. 3.2. Two different types of SAXS measurements are performed:

Static in-house SAXS measurements are employed to investigate the effect of small molecules on the assembly on vimentin wild type protein. These measurements are performed inside quartz glass capillaries.

Scanning-SAXS is employed to perform, combined with microfluidics, timeresolved measurements of vimentin wild type protein assembly and describes the exposure of the sample on multiple positions based on a defined mesh. The mesh is defined around the central region of the microfluidic device, where the vimentin wild type assembly occurs (see Fig. 4.6). Based on FEM simulations, reaction time points are calculated and correlated to the measured position inside the device. Measuring the SAXS signal at different positions inside the device corresponds to different reaction time points of the vimentin assembly process (see Sec. 5.2.1). All measurements are performed at room temperature.

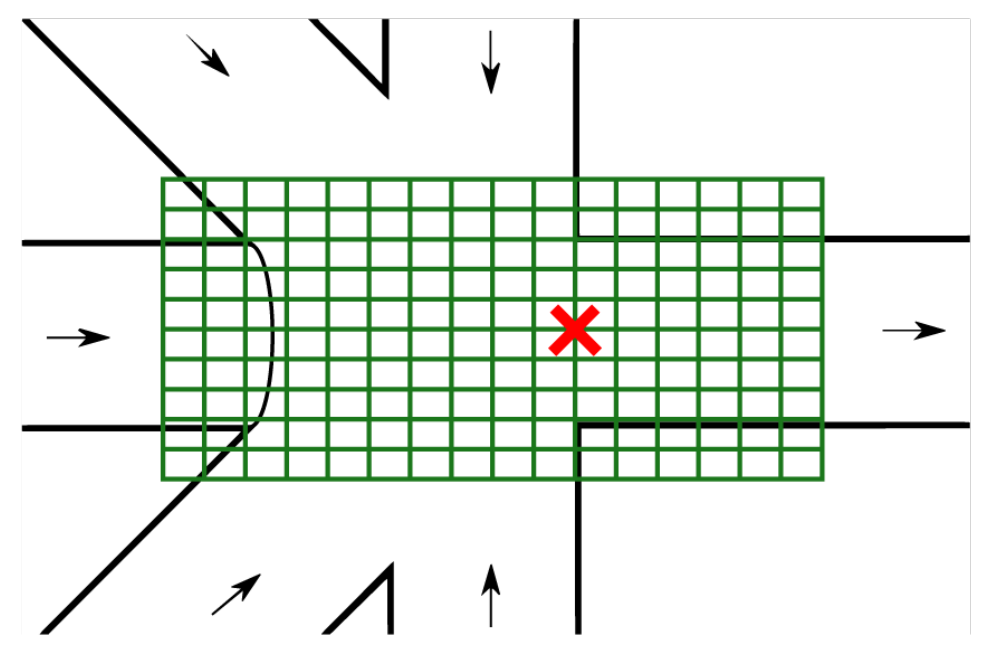

Figure 4.6.: Schematic representation of scanning SAXS experiments. A grid containing measurement points is defined around the central region of the microfluidic device (symbolised by the contour lines). The red cross represents the starting point from where the grid is defined. 


\subsubsection{Experimental Setup at cSAXS Beamline}

Time-resolved scanning SAXS measurements employing microfluidic devices are performed at the Coherent Small-Angle X-ray Scattering (cSAXS) beamline at the Swiss Light Source (SLS) at the Paul Scherrer Institute (PSI, Villigen, Switzerland). Electrons oscillating inside the undulator produce $X$-rays by Bremsstrahlung. The $\mathrm{X}$-rays are subsequently passed through a set of slits in order to sharpen the beam profile (see Figure 4.7). Photon energies of $11.2 \mathrm{keV}$ are selected using a Si(111) monochromator and are vertical focused by a bendable mirror. A second set of slits cleans the beam of parasitic scattering before the sample is illuminated inside a microfluidic device with a beam size of $10 \times 30 \mu \mathrm{m}^{2}$ (vertical $\times$ horizontal, full width at half maximum (FWHM)). To maximize the vimentin signal, the larger dimension of the beam is parallel to the flow of the microfluidic device. The device holder, with the microfluidic device mounted inside (see Sec. 4.2.2.2) is connected to a piezoelectric stage, allowing for precise movement during scanning SAXS measurements (see Sec. 4.3.3.2). Alignment of the sample in relation to the beam centre is performed using a calibrated visible light microscope. Behind the sample, the X-rays travel through an evacuated flight tube before being detected by a complementary metal-oxide-semiconductor detector (CMOS) located $7.11 \mathrm{~m}$ behind the sample (Pilatus2M detector, 1475 × 1679 pixels, pixel size: $172 \times 172 \mu \mathrm{m}^{2}$ ) [102]. A beamstop is placed directly before the detector to absorb the unscattered direct X-ray beam.

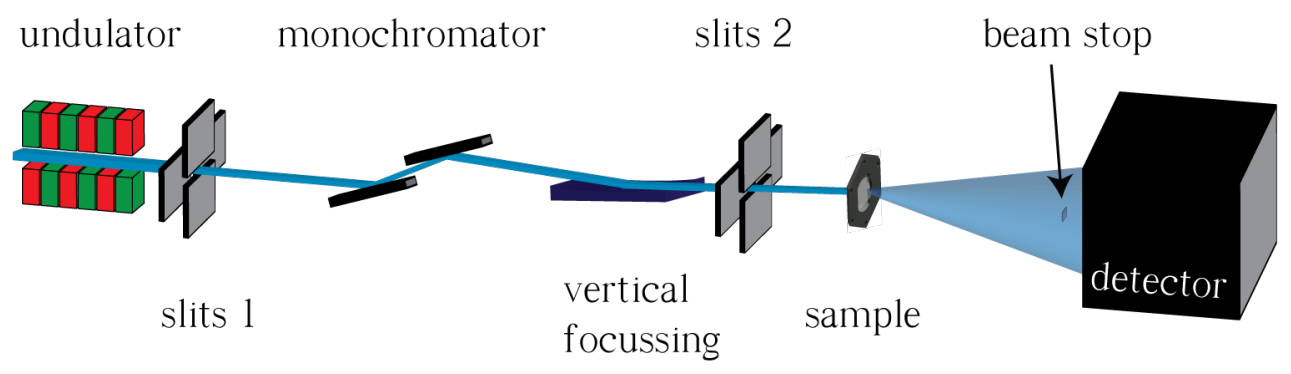

Figure 4.7.: Schematic of the beam bath at the cSAXS beamline. After being produced in the undulator X-ray photons pass a first set of slits and are energy-selected by a $\mathrm{Si}(111)$ monochromator. Subsequently bendable mirrors perform vertical focusing. Parasitic radiation is removed by a second set of slits. The sample is illuminated and the scattered photons are collected on a detector, whereas the unscattered photons are absorbed by a beam stop. 


\section{\begin{tabular}{l|l} 
Chapter 4 & EXPERIMENTAL METHODS
\end{tabular}}

\subsubsection{In-House Small-Angle X-Ray Scattering Setup}

Static SAXS measurements are performed on the commercially available in-house setup Xeuss 2.0 (Xenocs, Sassenage, France). The beam path is depicted in Fig. 4.8. The X-rays originate from a $\mathrm{Cu} \mathrm{K} \alpha^{-}$source $(\lambda=0.154 \mathrm{~nm})$ operating at $50 \mathrm{keV}$ and $600 \mu \mathrm{A}$. The source comprises beam collimation. After the source parasitic scattering of the collimated beam is removed by two pairs of scatterless slits. The collimation and the slits are adjusted to obtain a focus of the beam by approximately $0.5 \times 0.5 \mathrm{~mm}^{2}$. The sample is placed in an evacuated chamber. After the sample the beam enters an evacuated flight tube situated after the sample chamber to reduce air scattering. The focused beam is absorbed by a beam stop with a diameter of $3 \mathrm{~mm}$ placed before the exit window of the flight tube. The SAXS signal is recorded at $1.224 \mathrm{~m}$ from the sample by the detector (Pilatus3R1M, $981 \mathrm{x}$ 1043 pixels, pixel size $172 \times 172 \mu \mathrm{m}^{2}$, Dectris Ltd., Baden, Switzerland).

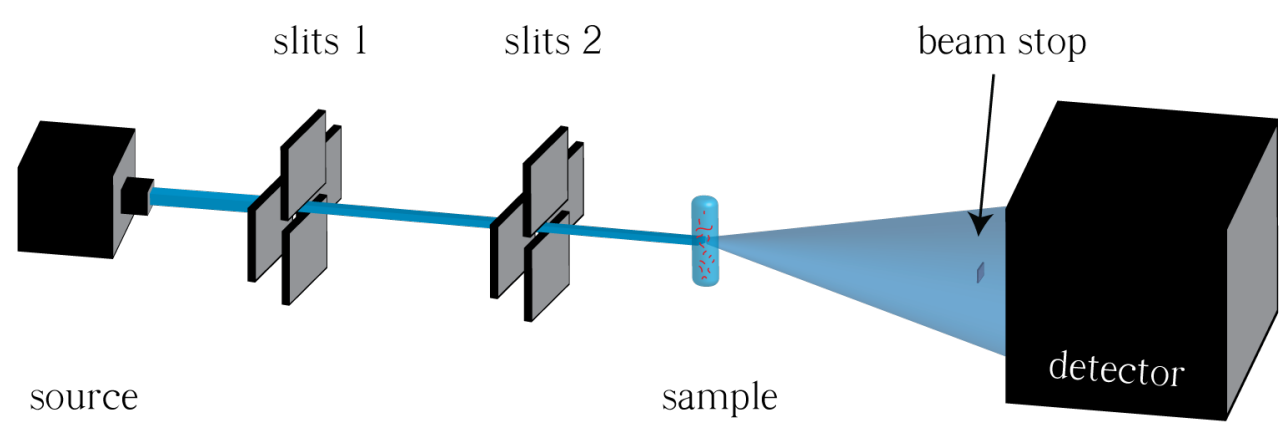

Figure 4.8.: Schematic of the in-house SAXS setup Xeuss 2.0. X-rays are produced by a Cu K-alpha source, focused and subsequently passed through two pairs of scatterless slits. After interacting with the sample, the direct beam is block by a beamstop, whereas the scattered photons are recorded on the detector. 


\subsubsection{Data Acquisition}

For both the static and scanning SAXS measurements the vimentin wild type protein is measured in solution, thus only small contrast between the sample and the surrounding solution is formed (see Sec. 3.1.4). To increase the sample signal, every measurement consists of multiple exposures for the same data point.

\subsubsection{Static Small-Angle X-Ray Scattering}

For static SAXS experiments the sample is measured inside quartz glass capillaries. Capillary widths are measured using a calliper and only capillaries with a similar outer diameter $d$ in the measuring region $(1.4 \mathrm{~mm}<d<1.6 \mathrm{~mm})$ are selected to ensure similar sample volumes for different experiments.

All further measurements steps are performed on the middle of the respective capillary and is distinguished by horizontally scanning the capillary and selecting the centre as the measuring point. The middle is selected to maximise the illuminated sample volume and to reduce edge effects due to the capillary. Capillaries are sealed with vacuum wax to prevent sample evaporation in the evacuated sample chamber. Short exposures without the beamstop are performed with and without the capillary to assure stability of the photon flux of the source and to account for the different transmission factor of the sample.

The exact thickness of the capillary is measured. To do so, the empty capillary is first measured and is subsequently filled with ultrapure water and remeasured to obtain the transmission factor. Both measurements are performed without a beam stop. Direct beams, i.e., the sample is removed from the photon beam, measurements are also recorded to account for possible fluctuations of the source.

The ultrapure water is replaced with buffer solution for the background measurement. To do so, the sealing vacuum wax is melted and removed from the capillary. The ultrapure water is replaced by the individual buffer solution and sealed again with vacuum wax. The buffer solution contains the exact ion concentrations as the sample solution later used for the subsequent sample measurements. Each capillary is measured for 12 hours, divided in $48 \times 900 \mathrm{~s}$ exposures. 4 capillaries are measured in a row, containing 3 different concentrations of either ACC or PBA and a control sample with neither ACC nor PBA.

The buffer solution is then exchanged, in the manner described above for vimentin solution, with sample solution containing $1 \mathrm{mg} / \mathrm{mL}$ vimentin wild type protein inside the buffer solution. After sealing the capillary with vacuum wax 


\section{\begin{tabular}{l|l} 
Chapter 4 & EXPERIMENTAL METHODS
\end{tabular}}

the ensemble is placed in an ultrapure water bath at $37^{\circ} \mathrm{C}$ for 4 hours to allow for the assembly of the vimentin (see Sec. 4.1.3). Subsequently, each capillary is again measured for 12 hours, divided in $48 \times 900$ s exposures. The initial and final scattering patterns are compared to assure no change occurred inside the sample or the buffer during the measurements.

\subsubsection{Time-Resolved Scanning Small-Angle X-Ray Scattering}

The device holder is assembled with a microfluidic device (see Sec. 4.2.2.2) and mounted to the piezoelectric sample stage at the cSAXS beamline. The sealed PE-tubings are opened and connected to the syringes containing the degassed buffers and the sample, which are mounted on the pump system (neMESYS, Cetoni $\mathrm{GmbH}$, Korbusen, Germany). A detailed description of the flow rates employed and the order of flow establishing is given in the appendix (see Sec. A).

A predefined spot of the mixing region within the microfluidic device is positioned in the beam centre and a mesh scan is performed (see Sec. 4.3). The mesh consists of $30 \times 62$ points (vertical $\times$ horizontal) in steps of $10 \mu \mathrm{m}$ and $25 \mu \mathrm{m}$, respectively. The distance of the points with respect to each other correlates with the beam size $\left(10 \times 30 \mu \mathrm{m}^{2}\right.$, vertical $\times$ horizontal, FWHM). Each point of the mesh is illuminated five times for 1 second each, while a constant flow of buffer solution is maintained. Vimentin wild type protein is subsequently flown into the chamber. The flow rates are then adapted in order to obtain the flow profile simulated in Sec. 5.2.1. After stabilization of the flow and verification that all air bubbles are eradicated, the scan is started. For both the buffer and sample measurements, identical scan points are exposed $(5 \times 1 \mathrm{~s})$.

\subsubsection{Data Treatment}

For static SAXS experiments the centre of the X-ray beam on the detector is calculated by recording a direct beam for $0.1 \mathrm{~s}$ and calculating the centre of mass. The sample-detector distance is calculated by exposing silver behenate powder placed between 2 Kapton sheets for several minutes to the X-ray beam. Comparing the recorded diffraction peaks to the well-known literature values yields the sample-detector distance [78]. Both steps are performed with the software Foxtrot, a software developed by Xenocs (Xenocs SAS, Grenoble, France) and Soleil (Synchrotron SOLEIL, Saint-Aubin, France).

The data processing for time-resolved scanning SAXS was carried out using the 
pixel mask

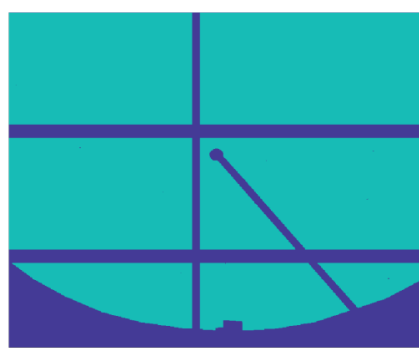

summed diffraction patterns

Figure 4.9.: A scattering image of glassy carbon is recorded and dead pixels, the shadow of the beamstop, the shadow of the flight tube and position of the detector panels are logically masked (left). Summed sample diffraction patters prior (middle) and after the application of the logic mask (right).

'cSAXS matlab package' developed by the Coherent X-ray Scattering Group (CXS group, Paul Scherrer Institut, Switzerland).

Before further analysis, dead pixels, the shadow of the beamstop, the shadow of the flight tube and position of the detector panel gaps are identified and logically masked from the recorded scattering image for every experiment. This procedure is depicted in Fig. 4.9 on a small region of the detector around the beamstop. Glassy carbon is illuminated by the X-ray beam before each set of measurements. The acquired scattering image is used to create a logic mask (left). This logic mask is subsequently applied to all recorded scattering images prior to the azimuthal integration (middle), resulting in not counting the pixel nor the pixel value for further analysis (right).

In order to distinguish the signal originating from the sample from its surrounding matter, for both the time-resolved and static SAXS measurements the scattering images recorded with the sample are background corrected. The principle of background subtraction for SAXS experiments is displayed in Figure 4.10. Scattering data are separately collected for both the sample container only containing buffer solutions (= background) and the sample container containing the sample in addition to buffer solutions (= sample and background, see Sec. 4.3.3). The recorded data are logically masked and azimuthally integrated resulting in the radial scattering intensity ( $I$ vs. q). Correction factors for each data point are calculated and applied to the radial scattering intensity (Fig. 4.10 left and middle). Obtaining the correction factor for static SAXS is described in Section 4.3.3.1 and the calculation 
sample and background

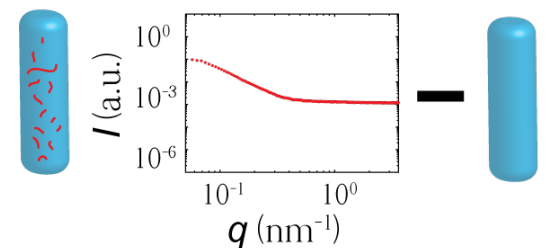

background

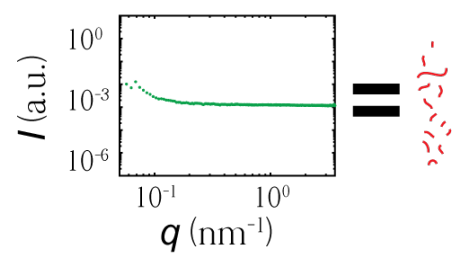

sample

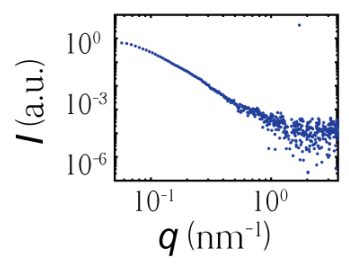

Figure 4.10.: Background subtraction in SAXS experiments. The sample container is measured first with buffer solution (middle). Subsequently the sample is added and the container is probed at the exact position again (left). By subtraction of the azimuthally integrated scattering patterns the sample signal is extracted (right).

of the correction factor for time-resolved scanning SAXS is described in Sec. 5.2.2. The radial scattering intensity of the sample is extracted (right) by subtracting the scaled, in respect to the flux and exposure time, radial scattering intensity of the background measurements (middle) from the scaled radial scattering intensity of the sample and background measurement (left).

\subsection{Fluorescence Imaging of Vimentin Assembly}

Epi-fluorescence microscopy imaging is performed with an inverted microscope (IX81, Olympus, Tokyo, Japan). Images are recorded with a CMOS camera (Orca flash 4.0 LT- C11440-42U, Hamamatsu Photonics Deutschland GmbH, Herrsching am Ammersee, Germany) with an effective pixel number $2048 \times 2048$ pixels. The fluorescence signal of the ATTO $647 \mathrm{~N}$ labelled vimentin (see Sec. 4.1.2) is captured using a 100x oil immersion objective (UPlanSApo, Olympus, Tokyo, Japan) immersed in a low autofluorescence immersion oil (IMMOIL-F30CC, Olympus, Tokyo, Japan). The objective has an numerical aperture of 1.40. Kick-started vimentin wild-type protein (see Sec. 4.1.3) is diluted 1:100 in buffer containing the identical buffer conditions as during the kickstart. After dilution, $7.5 \mu \mathrm{L}$ is pipetted between two cover slips (VWR, Radnor, PA, USA) and directly imaged at room temperature. The exposure time is set to $500 \mathrm{~ms}$ per image. This procedure is repeated for each sample at every time point and concentration. 


\section{Results}

The main results of the conducted experiments are presented in this chapter. In the first part, data published as an article in Lab on a Chip [97] is presented. The second part presents results of time-resolved vimentin assembly recorded in COC step devices, with an emphasis on simulating the flow inside the device and tools developed for data analysis. In the third part the influence of small chemical compounds on the assembly of vimentin are described. A structure detection algorithm developed for analysing microscopy images is presented and using epifluorescence microscopy and static-SAXS the effects of ACC and PBA on vimentin assembly are quantified. In a last part the results for both chemical compounds investigated are compared and contextualized with reported results. 


\subsection{Cyclic Olefin Copolymer as an X-Ray Compatible Ma- terial for Microfluidic Devices}

\subsubsection{Preamble}

The original article is accessible under 'Cyclic olefin copolymer as an X-ray compatible material for microfluidic devices' , M. Denz and G. Brehm et al., Lab Chip, 18, 171-178 (2018). DOI: 10.1039/C7LC00824D [97]. The experimental work for this article was performed during my master thesis.

Susanne Bauch prepared buffers, produced and isolated the vimentin. Manuela Denz and I adapted the protocol for NOA-Kapton devices. I produced several of the NOA-Kapton devices. I adapted the COC protocol and produced the COC devices. Together with Manuela Denz I provided the images for Fig. 1, 2 and S1. I performed simulations shown in Fig. 2. I made Fig. 3, S2 and S3. Manuela Denz prepared buffers and vimentin for the colloid experiments and produced most of the NOA-Kapton devices. Manuela Denz performed the data analysis and made Fig. 1, 2, 4, 5 and S1. Clément Y. J. Hémonnot developed with Heidi Spears a first COC device fabrication protocol and provided MATLAB scripts based on a script by Oliver Bunk (Paul Scherrer Institute, Villigen, Switzerland). Andrew Wittmeier, Chiara Cassini, Oliva Saldanha and Eleonora Perego helped during the experiments. Ana Diaz and Manfred Burghammer provided technical support as beamline scientist at SLS and ESRF, respectively. Sarah Köster designed the research. Manuela Denz and I wrote a first version of the manuscript prior to iterative optimization with Sarah Köster, Manuela Denz and myself. All authors commented on the manuscript. 
Check for updates

Cite this: Lab Chip, 2018, 18, 171

\title{
Cyclic olefin copolymer as an X-ray compatible material for microfluidic devices $\uparrow$
}

\author{
Manuela Denz, + ${ }^{\mathrm{a}}$ Gerrit Brehm, ${ }^{\mathrm{ab}}$ Clément Y. J. Hémonnot, ${ }^{\mathrm{a}}$ Heidi Spears, ${ }^{a}$ \\ Andrew Wittmeier, ${ }^{a}$ Chiara Cassini, ${ }^{a}$ Oliva Saldanha, ${ }^{a}$ Eleonora Perego, ${ }^{a}$ Ana Diaz, ${ }^{c}$ \\ Manfred Burghammer ${ }^{d}$ and Sarah Köster (iD *ab
}

\begin{abstract}
The combination of microfluidics and X-ray methods attracts a lot of attention from researchers as it brings together the high controllability of microfluidic sample environments and the small length scales probed by $\mathrm{X}$-rays. In particular, the fields of biophysics and biology have benefited enormously from such approaches. We introduce a straightforward fabrication method for $\mathrm{X}$-ray compatible microfluidic devices made solely from cyclic olefin copolymers. We benchmark the performance of the devices against other devices including more commonly used Kapton windows and obtain data of equal quality using small angle $\mathrm{X}$-ray scattering. An advantage of the devices presented here is that no gluing between interfaces is necessary, rendering the production very reliable. As a biophysical application, we investigate the early time points of the assembly of vimentin intermediate filament proteins into higher-order structures. This weakly scattering protein system leads to high quality data in the new devices, thus opening up the way for numerous future applications.
\end{abstract}

Accepted 28th November 2017

DOI: $10.1039 /$ c7lc00824d

rsc.li/loc microfluidic devices, such as concentration gradients, specific flow fields, or diffusive mixing in laminar flow. As a result, time resolved investigations of reactions and mechanisms, which cannot be captured by light microscopy, due to the relevant length scales of the system, can be performed.

The success of these applications is further highlighted by recent and planned developments concerning synchrotron light sources. Highly focused and brilliant beams are well compatible with small channel dimensions and time-resolved measurements. However, as recently pointed out by Ghazal et $a l .{ }^{2}$ there is still a need for versatile, reproducible X-ray compatible microfluidic devices, which may be fabricated and used by a large community of scientists beyond the immediate group of specialists. The most important requirements for the employed materials are moldability, X-ray resistance, transparency to X-rays and a low background scattering signal. To achieve these characteristics, many design approaches have utilized combinations of different materials, including etched silicon with PDMS windows, ${ }^{14-18}$ PDMS and quartz glass capillaries, ${ }^{23,25}$ PDMS and Kapton film, ${ }^{26,27}$ stainless steel and Kapton film, ${ }^{13,20,22,26,28}$ optical adhesive $^{21,23}$ and optical adhesive with Kapton film and silicon rich nitride windows. ${ }^{29}$ Fabrication of these devices typically involves advanced engineering skills.

In X-ray crystallography, another commonly used material class are cyclic olefin copolymers (COCs). Steigert et al. introduced fabrications methods for COC devices ${ }^{30}$ which were used for miniaturized and highly parallelized protein 
crystallization under well defined conditions. ${ }^{31}$ Similar approaches, including screening of crystal growth and data collection inside the device, have been reported in the following years. $^{32-35}$ These devices used a combination of COC and PDMS to allow for the inclusion of microfluidic valves and used silanes to bond the layers. A SAXS study in a commercial all-COC device was performed on liquid crystals, which typically scatter considerably stronger than proteins. ${ }^{36}$ Silane based binding was found to be insufficient between two COC layers ${ }^{37}$ and thus, instead, a thin layer of COC was plasticized by solvent before bonding to the second layer. ${ }^{37,38}$ Alternatively, thermal sealing of layers with different glass transition temperatures $T_{\mathrm{G}}$ was reported. ${ }^{30,39}$ Importantly, when using heat and pressure for sealing, the thickness of the finished device depends on the applied temperature and pressure due to changes in viscosity of the COC with temperature. ${ }^{37}$

COCs are well suited for X-ray applications due to the low photon absorption at relevant photon energies between 2 and $20 \mathrm{keV}$, as compared to PDMS and $\mathrm{SiO}_{2} \cdot{ }^{33}$ Furthermore, COCs are optically transparent and provide resistance against many solvents. ${ }^{40}$

Here, we adapt COCs for SAXS measurements on weakly scattering protein and present the reproducible fabrication and thorough characterization of an easy-to-manufacture, leak-tight, low scattering and customizable microfluidic device. As the device is made solely out of one type of COC, with similar glass transition temperature, it displays no interfaces whatsoever and we circumvent additional plasticization steps. The manufacturing process is based on standard soft lithography and well established molding techniques, combined with hot embossing. Lateral channel structures can flexibly be adapted, due to the lithographic approach. We characterize the devices using gold nanoparticles and, thereafter, demonstrate the utility by recording protein SAXS data.

\section{Materials and methods}

\section{Device fabrication and usage}

Channel structures were produced using standard soft lithography methods. ${ }^{1,41}$ Briefly, SU-8 2150 negative photo resist (MicroChem, Newton, USA) was spin-coated onto a 2-inch silicon wafer (Si-wafer; MicroChemicals, Ulm, Germany) to a final height of $160 \mu \mathrm{m}$. Edge bead removal was performed before the soft bake step to achieve an ideal contact between the photo mask (Selba S.A., Versoix, Switzerland) and the substrate. The resist was exposed to UV light (365 nm) using a mask-aligner (MJB4 Mask-Aligner, Süss MicroTec AG, Garching, Germany) through the mask and the structure was developed after a post exposure step (see Fig. 1a). Next, we produced a PDMS (Dow Corning, Midland, USA) copy of the channel structure (see Fig. 1b). The PDMS copy was then used as a stamp to obtain a copy of our channel structure made from UV-curable adhesive (NOA 81, Norland Optical Adhesives, Cranbury, NJ, USA) on a glass slide as support (see Fig. 1c). The PDMS was pushed into the UV-curable adhesive, which was then cured under a UV-lamp (366 nm, a

Si-wafer

b
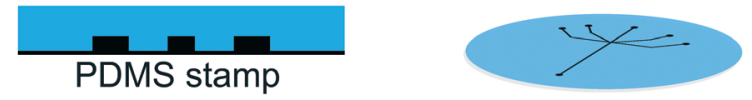

C
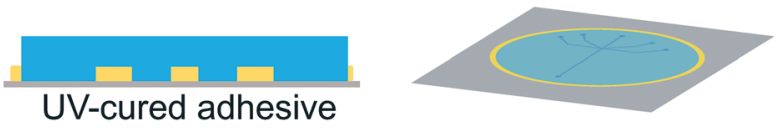

d structure in $\mathrm{COC}$

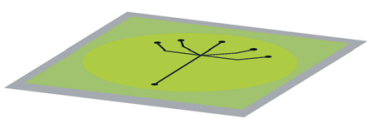

\section{finished device}

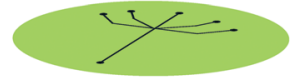

Fig. 1 Manufacturing steps of a COC device. (a) Channel structures were fabricated by photolithography (black) using SU-8 2150 negative photo resist spin coated on Si-wafers. (b) PDMS copies (blue) of the channel structures were produced. (c) By pressing the PDMS into UVcured adhesive (yellow) deposited on a glass slide, channel structures were replicated. (d) COC sheets (green) were heated above the glass transition temperature, and structured by hot embossing. (e) A COC sealing layer was used to close the channels.

NU-8 KL. Benda, Wiesloch, Germany) for 3 minutes. Subsequently, the PDMS stamp may be easily removed from the glass slide, leaving behind a channel structure in UV-curable adhesive. Excess adhesive was cut away with a scalpel. Afterwards, the structure was exposed to UV light for additional 3 minutes. Note that the obtained UV-curable adhesive stamp on the glass slide can be reused. A $240 \mu \mathrm{m}$ thick layer of COC (msc foil 029, Microfluidic ChipShop GmbH, Jena, Germany), with a glass transition temperature $T_{\mathrm{G}}$ of $78{ }^{\circ} \mathrm{C}$, was placed on top of the channel structure on the glass slide. The ensemble was sandwiched between two sheets of paper, followed by two sheets of aluminum foil and placed in the preheated hot press ( $\mathrm{PW} 10 \mathrm{H}, \mathrm{P} / \mathrm{O} /$ Weber $\mathrm{GmbH}$, Remshalden, Germany) at $130{ }^{\circ} \mathrm{C}$ for 5 minutes. The ensemble was subsequently pressed with initially $2.2 \mathrm{kN}$ for $10 \mathrm{mi}-$ nutes (see Fig. 1d). After cooling down, the COC structure was removed from the UV-curable adhesive structure and holes were punched for the inlets and outlet of the microfluidic device using $0.5 \mathrm{~mm}$ biopsy punchers (Harris UniCore $^{\mathrm{TM}}$ puncher, Plano, Wetzlar, Germany). Finally, the channel structure was sealed with a $20 \mu \mathrm{m}$ thick layer of COC (Topas $8007 T_{\mathrm{G}}=75{ }^{\circ} \mathrm{C}$, TOPAS Advanced Polymers $\mathrm{GmbH}$, Frankfurt, Germany) via a heat and pressure controlled lamination machine (Catena 35 GBC, ACCO Brands, Lincolnshire, USA) at $100{ }^{\circ} \mathrm{C}$, a speed of $7.6 \times 10^{-3} \mathrm{~m} \mathrm{~s}^{-1}$ and narrowest setting for the rolls. For better handling, the device and the sealing layer were placed between two sheets of paper during 
sealing. The resulting microfluidic channels were measured using optical microscopy to $200 \mu \mathrm{m}$ width and $160 \mu \mathrm{m}$ height (Fig. 1e). For a more detailed figure with all assembly steps, see Fig. S1. $\dagger$ The measurement window consisted of COC with a thickness of about $50 \mu \mathrm{m}$ and additional $20 \mu \mathrm{m}$ sealing layer (see Fig. S2 $\dagger$ ). Note that during heat and pressure treatment the COC layer becomes thinner, thus accounting for the "missing" $30 \mu \mathrm{m} .{ }^{37}$

UV-curable adhesive/Kapton devices were fabricated as previously described. ${ }^{29}$ Briefly, a PDMS copy was produced from the Si-wafer. Afterwards, a second PDMS stamp was produced from the first PDMS copy. An $8 \mu \mathrm{m}$ thick Kapton foil (SPEX SamplePrep, Metuchen, NJ, USA) with liquid adhesive (NOA 81) was prepared on an aluminum block. Adhesive was also pipetted on the PDMS structure and placed in a desiccator for 15 minutes to remove air bubbles. Subsequently, the PDMS structure was pressed into the adhesive on the Kapton foil to obtain the channel structure. After partially curing the adhesive, holes for the inlets and outlet were punched, using a biopsy puncher with a diameter of $0.75 \mathrm{~mm}$. The PDMS stamp was removed and a second Kapton foil was used to seal the device, which contained measurement windows of 16 $\mu \mathrm{m}$ Kapton in total and possibly a thin layer of UV-cured adhesive. In the finished device, the channels had a width of $200 \mu \mathrm{m}$ and a height of $160 \mu \mathrm{m}$.

As a geometry for all devices, we chose a five-inlet/one-outlet system with $45^{\circ}$ angles between the inlet channels (Fig. 2a). Through the central inlet, protein $\left(5.5 \mathrm{mg} \mathrm{mL}^{-1}\right)$ or gold colloid solution (Sigma Aldrich, St. Louis, Missouri, USA; radius $R=15 \mathrm{~nm}$, concentration in the syringe $c=1.8 \times$ $10^{11}$ particles per $\mathrm{mL}$ and $R=10 \mathrm{~nm}, c=6.5 \times 10^{11}$ particles per $\mathrm{mL}$ ) was injected at a rate of $15 \mu \mathrm{L} \mathrm{h}^{-1}\left(1.3 \times 10^{-4} \mathrm{~m} \mathrm{~s}^{-1}\right)$. Sheath inlets, ${ }^{42}$ which were situated on both sides of the central inlet, were injected with buffer at a low flow rate of 7.5 $\mu \mathrm{L} \mathrm{h}^{-1}\left(6.5 \times 10^{-5} \mathrm{~m} \mathrm{~s}^{-1}\right)$. The side inlets contained $110 \mathrm{mM}$ $\mathrm{KCl}$ in buffer for protein experiments and phosphate buffered saline (PBS) for gold colloid experiments at a flow rate of $150 \mu \mathrm{L} \mathrm{h}^{-1}\left(1.3 \times 10^{-3} \mathrm{~m} \mathrm{~s}^{-1}\right)$.

Polyethylene tubing (inner diameter $0.38 \mathrm{~mm}$, outer diameter $1.09 \mathrm{~mm}$, Intramedic Clay Adams Brand, Becton Dickinson and Company, Sparks, USA) was connected to Hamilton Gastight glass syringes (Bonaduz, Switzerland) with $1 \mathrm{~mL}$ or $0.5 \mathrm{~mL}$ volume by disposable needles. Syringe pumps (neMESYS, Cetoni GmbH, Korbußen, Germany) were employed for precise flow control over all five inlets. A sample holder based on the one introduced in Urbani's dissertation, ${ }^{43}$ was used to tightly connect the tubing and device in a leak-free manner. Briefly, the sample holder consisted of two metal plates that sandwich one PVC (polyvinylchloride) plate and the device itself. The metal and PVC plates were equipped with an opening that served as a measurement window. Tubing was threaded through small holes in the front metal and the PVC plate as well as o-rings for sealing. The device was aligned with the tubing and fixed to the PVC plate with sticky tape. The whole ensemble was screwed onto the metal back plate, which was larger then the other plates a

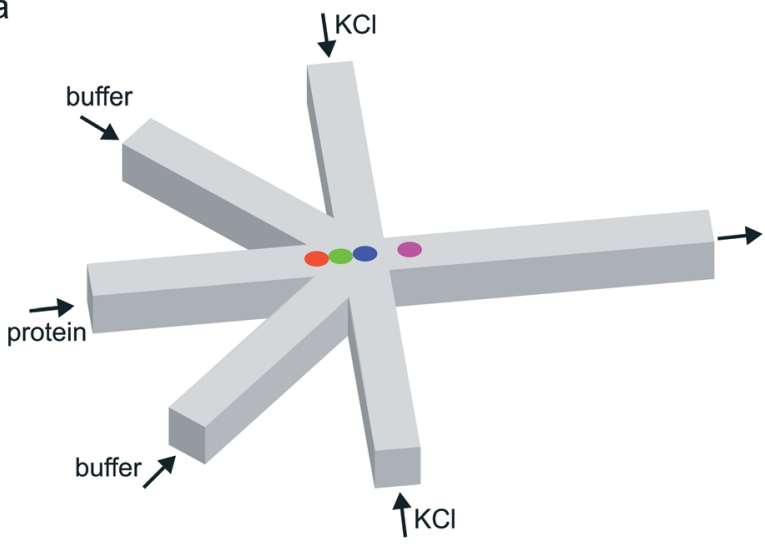

b

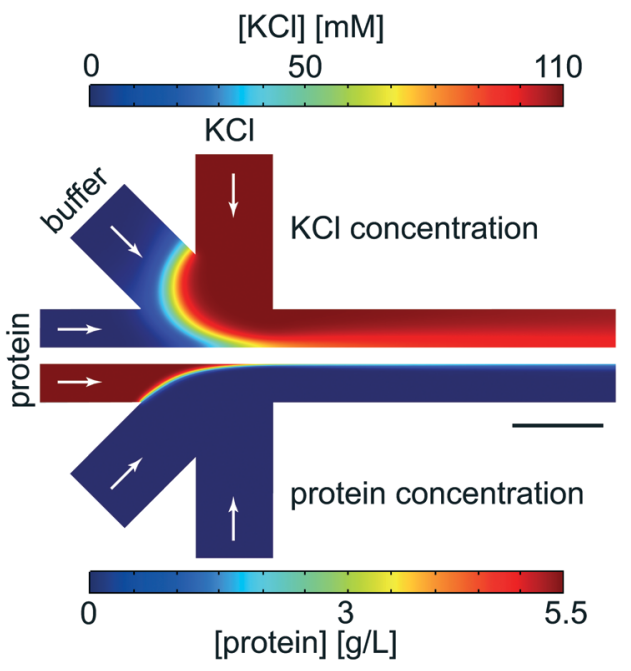

C

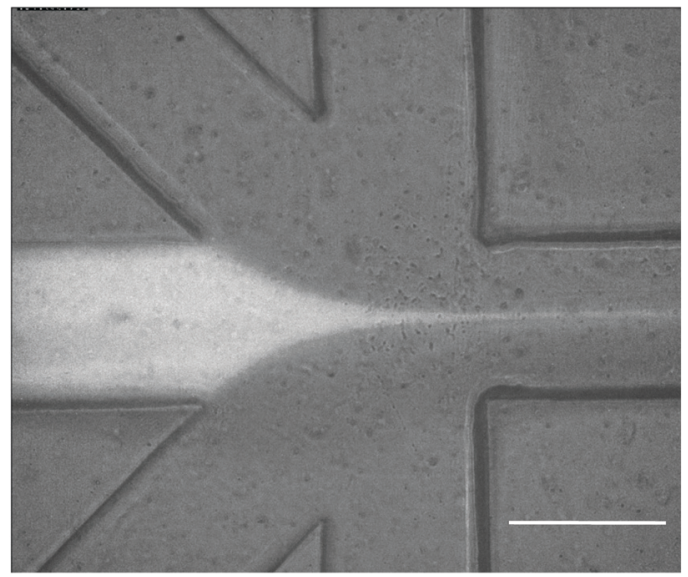

Fig. 2 Geometry of the microfluidic device. (a) Scheme of the device layout. The sample (protein or colloid solution) was injected through the central inlet. The side inlets were used for injecting assembly start buffer with $\mathrm{KCl}$ for vimentin, or buffer for gold experiments. The diagonal sheath buffer inlets push the mixing point further into the outlet channel. The colored dots refer to the positions analyzed in the vimentin experiment (see Fig. 5). (b) Results of the FEM flow simulations: concentration of $\mathrm{KCl}$ (upper half of the figure) and vimentin (lower half of the figure). (c) Fluorescence and bright field micrograph (overlay) of microfluidic experiment performed with labeled vimentin (Alexa 488). All scale bars $200 \mu \mathrm{m}$. 
and served as an adapter plate to the beamline sample stage. A photograph of the sample holder used for the experiments is shown in Fig. S3.†

\section{Protein purification and assembly}

Human vimentin protein with a molecular weight of 53.7 kDa was expressed in Escherichia coli bacteria and then purified from inclusion bodies. ${ }^{44}$ Vimentin was stored at $-80{ }^{\circ} \mathrm{C}$ in $8 \mathrm{M}$ urea, $5 \mathrm{mM}$ Tris-HCl (pH 7.5), $1 \mathrm{mM}$ EDTA, $0.1 \mathrm{mM}$ EGTA, $1 \mathrm{mM}$ DTT and $10 \mathrm{mM}$ methyl ammonium chloride (MAC). The purity of the protein was verified by SDSpolyacrylamide gel electrophoresis. All solutions were prepared using $2 \mathrm{mM}$ phosphate buffer (PB), $\mathrm{pH}$ 7.5. Before use in the experiments, the protein was dialyzed against $8 \mathrm{M}$ urea for 30 minutes and then, in a stepwise manner, against 6 , 4,2 , and $1 \mathrm{M}$ urea for 30 minutes each, at room temperature. Finally, an overnight dialysis into $2 \mathrm{mM}$ PB was performed at $10{ }^{\circ} \mathrm{C}$. All dialysis steps were performed using membranes of $50 \mathrm{kDa}$ cut-off (SpectraPor, Carl-Roth GmbH, Karlsruhe, Germany). The protein concentration was determined to be $5.5 \mathrm{mg} \mathrm{mL}^{-1}$ by measuring the absorption at $280 \mathrm{~nm}$ (Nanodrop ND-1000, ThermoScientific Technologies, Inc., Wilmington, USA). Assembly was initiated in the microfluidic devices by diffusive addition of $110 \mathrm{mM} \mathrm{KCl}$ in buffer to the vimentin solution. The calculated end concentration of $\mathrm{KCl}$ after complete mixing in the outlet channel was $100 \mathrm{mM}$, and the vimentin concentration at the last measured position shown here was $4.0 \mathrm{mg} \mathrm{mL}$. For microscopy experiments in a UV-curable adhesive/Kapton device, AlexaFluo 488 (Invitrogen GmbH, Darmstadt, Germany) labeled vimentin was used. ${ }^{45,46}$ An inverted Olympus IX71 microscope (Olympus Europa SE \& CO. KG, Hamburg, Germany) equipped with a $10 \times$ objective was employed to image the flow. Flow rates in this experiment were $100 \mu \mathrm{L}$ $\mathrm{h}^{-1}\left(8.7 \times 10^{-4} \mathrm{~m} \mathrm{~s}^{-1}\right)$ for the side inlets $(100 \mathrm{mM} \mathrm{KCl}), 10$ $\mu \mathrm{L} \mathrm{h}^{-1}\left(8.7 \times 10^{-5} \mathrm{~m} \mathrm{~s}^{-1}\right)$ for the central protein inlet and 5 $\mu \mathrm{L} \mathrm{h}^{-1}\left(4.3 \times 10^{-5} \mathrm{~m} \mathrm{~s}^{-1}\right)$ for the sheath inlets.

\section{Small angle $X$-ray scattering}

SAXS measurements were performed at two different synchrotron sources, the ID13 beamline at the European Synchrotron Radiation Facility (ESRF) (Grenoble, France) and the cSAXS beamline at the Swiss Light Source (SLS) (Paul Scherer Institut, Villigen, Switzerland). All measurements were performed at room temperature and in air.

At ID13, an in-vacuum undulator was used and the energy (13.9 keV) was selected using an $\mathrm{Si}(111)$ monochromator. A transfocator in vacuum guaranteed reproducible changing of the X-ray lens packages. We used beryllium compound refractive lenses composed of 54 individual lenses with parabolic shape and a radius of curvature of $200 \mu \mathrm{m}$ in the apex. Three apertures with $50 \mu \mathrm{m}, 20 \mu \mathrm{m}$ and $80 \mu \mathrm{m}$, respectively, were included in the beam path. For background reduction, a 70 mm long flight tube with silicon nitride entrance window and polypropylene exit window filled with helium was employed. As a beamstop, a lead cylinder with a few $\mathrm{mm}$ in length and about $300 \mu \mathrm{m}$ in diameter was installed. An Eiger $4 \mathrm{M}$ detector $\left(2070 \times 2167\right.$ pixels, pixel size $75 \times 75 \mu^{2}$, Dectris, Baden, Switzerland $)^{47}$ was placed at $0.95 \mathrm{~m}$ from the sample. Experiments were performed at a primary beam intensity of $8 \times 10^{11} \mathrm{ph} \mathrm{s}^{-1}$ with a beam size of $2.7 \times 1.7 \mu \mathrm{m}^{2}$ and an exposure time of $1 \mathrm{~s}$.

At cSAXS, the sample was placed at a distance of $33.8 \mathrm{~m}$ from the undulator source. The beam was defined by a set of slits of $0.6 \mathrm{~mm} \times 0.4 \mathrm{~mm}$ (horizontal $\times$ vertical) at $26.0 \mathrm{~m}$ from the source. An energy of $11.2 \mathrm{keV}$ was selected with a double-crystal $\mathrm{Si}(111)$ monochromator placed at about 28.5 $\mathrm{m}$ from the source, which was also used for horizontal focusing. A bendable mirror at $29.4 \mathrm{~m}$ from the source further focused the beam vertically. Different sets of slits downstream of the X-ray optics were used to clean up the beam from parasitic scattering. In order to optimize the beam size along the horizontal direction, we used a pair of slits with a horizontal aperture of $0.1 \mathrm{~mm}$ placed at $12.1 \mathrm{~m}$, which helped to effectively decrease the source size but reduced the flux to about $50 \%$. The beam was focused at the sample position, where we measured a beam size of $12 \times 29 \mu \mathrm{m}^{2}$ with an estimated intensity of $2 \times 10^{11} \mathrm{ph} \mathrm{s}^{-1}$. The sample scattering was recorded with a Pilatus $2 \mathrm{M}$ detector $(1475 \times 1679$ pixels, pixel size: $172 \times 172 \mu^{2}$, SLS detector group, Villigen, Switzerland $)^{48}$ at a sample-to-detector distance of $7.087 \mathrm{~m}$. An evacuated flight-tube was placed between the sample and the detector to reduce parasitic air scattering. The total exposure time was $5 \mathrm{~s}$, split up in $5 \times 1 \mathrm{~s}$.

For a pointwise background subtraction, we performed one measurement on a buffer-filled device and directly afterwards, in the same device, the actual measurement, including the sample. By this approach, we obtained a background measurement in every position of the device, thus accounting for variations between devices and between positions in the same device.

\section{Data analysis}

Measurements were performed on a mesh located at the channel cross section, as schematically shown in Fig. 3a. Dark-field images were calculated by integrating the complete scattered intensity in each measurement position and plotting it on a color scale, see Fig. $3 \mathrm{~b}$, in which the focused flow stream can easily be located. Each 'pixel' in the dark-field image represents a full 2D scattering pattern (Fig. 3c), which was azimuthally integrated. After integration, a pointwise background subtraction was performed. The calculated intensity $I(q)$ was then plotted against the magnitude of the scattering vector

$$
q=\frac{4 \pi}{\lambda} \sin (\theta),
$$

where $\theta$ is half the scattering angle and $\lambda$ is the wavelength of the radiation. Guinier analysis of the data was performed using the software package PRIMUS (ATSAS, EMBL, 
a

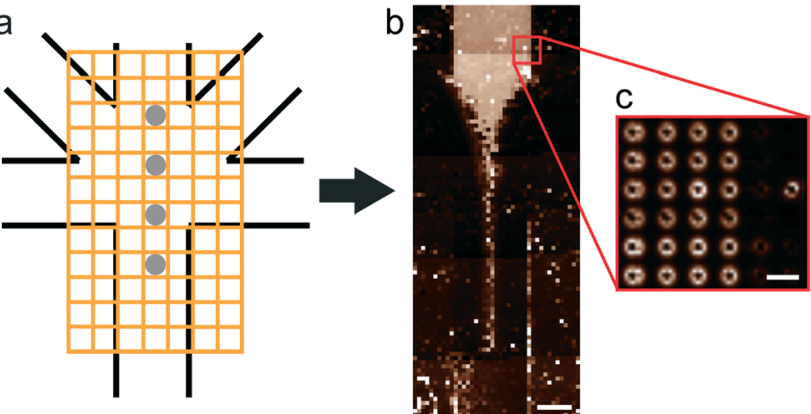

Fig. 3 Data acquisition and analysis. (a) Scheme of data acquisition in a mesh geometry (not to scale), the gray circles indicate typical analyzed positions. (b) The whole mesh is displayed in a dark-field image with the total scattered intensity in each position plotted on a color scale (typical gold colloid measurement in a COC device at ID13). (c) Beyond the intensity value plotted in b, every position contains a full 2D scattering pattern, which was further analyzed by radial integration. Scale bars are 100 and $10 \mu \mathrm{m}$, respectively.

Hamburg, Germany) ${ }^{49}$ to retrieve the overall size of the scatterers by determining the radius of gyration $R_{\mathrm{g}}$ for solid spheres:

$$
\ln I(q)=\ln I(0)-\frac{R_{\mathrm{g}}{ }^{2}}{3} q^{2}
$$

Furthermore, the pair distance distribution function $p(r)$ (pddf) was used to validate the results:

$$
R_{\mathrm{g}}^{2}=\frac{\int_{0}^{D_{\max }} r^{2} p(r) \mathrm{d} r}{2 \int_{0}^{D_{\max }} p(r) \mathrm{d} r} .
$$

The pddf is the distribution of distances between scatterers in a sample. An advantage of the pddf is that it takes the whole scattering curve into account and not only the first few data points as in Guinier's approximation. In the third step, we fitted the form factor $P(q, R)$ using Matlab2017a (The MathWorks, Natick, MA, USA) for solid spheres. The form factor can be written as

$$
P(q, R)=\left[\frac{3 \sin (q R)-q R \cos (q R)}{q R^{3}}\right]^{2} .
$$

This approach also takes the whole curve into account and the fit curve can be compared to the experimental data.

\section{Flow simulations}

COMSOL Multiphysics 5.2a (COMSOL GmbH, Göttingen, Germany) was used to perform finite element method (FEM) simulations of the flow conditions in the microfluidic devices. The inlet channels were reduced in length in the model to reduce the computational time, but are long enough to ensure equilibrated flow and mixing profiles. Simulations were performed in the laminar flow regime, thus employing the time-independent Navier-Stokes-equation, and no-slip boundary conditions are used at the channel walls. FEM simulations shown here represent the protein experiments using the diffusion coefficients $D_{\text {vim }}=2.2 \times 10^{-11} \mathrm{~m}^{2} \mathrm{~s}^{-1}$ and $D_{\mathrm{KCl}}=$ $1.84 \times 10^{-9} \mathrm{~m}^{2} \mathrm{~s}^{-1}$. The diffusion coefficient for vimentin is an estimate of the upper bound, since it decreases along the channel as the protein assembles. A change in viscosity upon assembly is not included in our simulation, since viscometry measurements show that the viscosity increases only very little during the first seconds of the assembly process. ${ }^{50,51}$

\section{Results and discussion}

\section{Device characterization}

Kapton was chosen as a "base line" for our comparison, because the material is well established for X-ray experiments. $^{8-10,13,22,26,29}$ This is due to its low and well defined background signal and negligible radiation sensitivity. Thus, Kapton provides a number of advantages when used as window material. However, in a typical microfluidic chamber, Kapton films have to be glued or bonded to other materials, such as stainless steel plates, ${ }^{8-10,13,22}$ PDMS $^{26}$ or UV-curable adhesive ${ }^{29}$ that are used for defining the channel geometry, because the material is not self-adhesive. Kapton film with an adhesive layer ("Kapton tape") typically leads to a fairly strong background signal itself. An exception are devices produced by laser ablation directly in Kapton and bonded to thermo-sensitive Kapton, ${ }^{52}$ which require considerable efforts in fabrication. The COC devices we present here, by contrast, are made from one single material such that there are no (glued) interfaces between the layers. We bond the COC layers directly without using solvents or silanes and without an additional plasticization step.

To benchmark the new devices and test if they are suitable for SAXS measurements in general and in particular for SAXS on weakly scattering protein, we here directly compare measurements in microfluidic devices comprising $70 \mu \mathrm{m}$ COC windows or $16 \mu \mathrm{m}$ Kapton windows (total thickness). As a defined test system, gold colloids were used and we performed the experiments at two different synchrotron beamlines to account for differences concerning primary setup geometry, beam intensity, beam size and detectors. Fig. 4a shows typical scattering curves of gold colloids in the two device types, measured at ID13, ESRF. An advantage of the beamline is the highly focused beam $\left(2.7 \times 1.7 \mu \mathrm{m}^{2}\right)$, which can be used to exactly map the microfluidic channel. The background subtracted scattering data in the COC device (green) and in the Kapton device (blue) agree very well, even concerning the scattering intensity values. To further benchmark the validity of the data, we performed an analysis of the pddf yielding a radius of $R=11.02 \mathrm{~nm} \pm 0.15 \mathrm{~nm}$. Furthermore, the form factor of solid spheres was fitted to the data (red solid line) and we obtain a radius of $10.55 \mathrm{~nm} \pm$ $0.06 \mathrm{~nm}$.

The cSAXS beamline at SLS is optimized for SAXS, providing access to very low $q$-values. Again, the data curves for the COC and the Kapton device fall exactly onto each other, as 
a

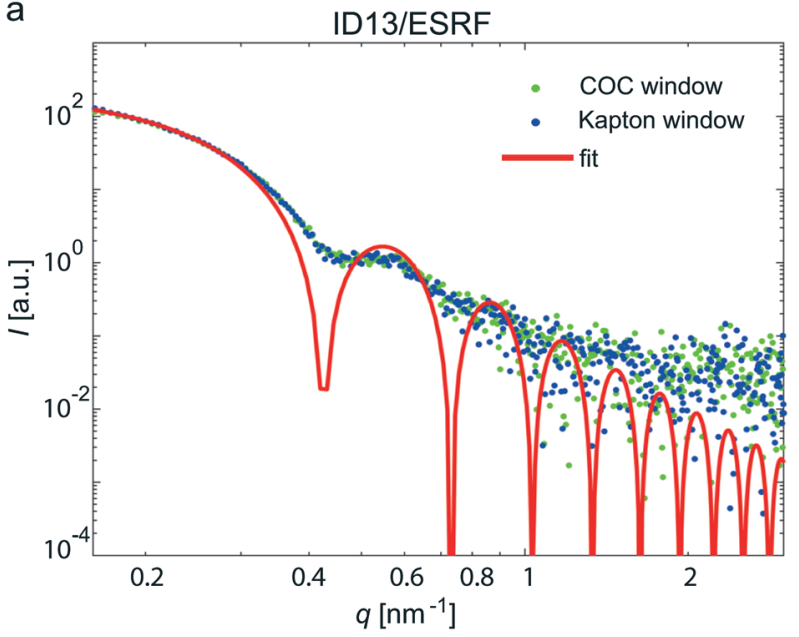

b

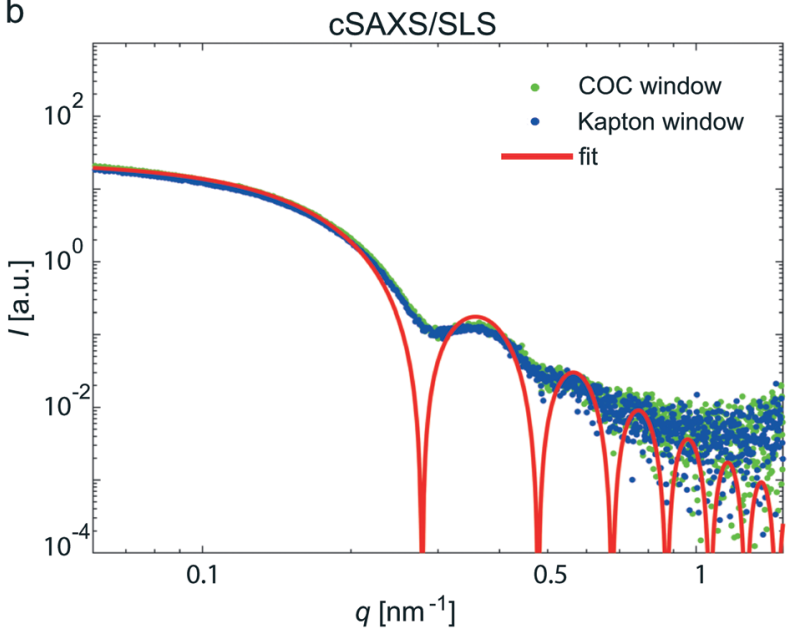

Fig. 4 Colloid scattering curves with corresponding form factor. (a) Gold colloids measured at the ID13 beamline (ESRF) in a device with a $\mathrm{COC}$ window (green) and a Kapton window (blue). The form factor for solid spheres was fitted to the data and we obtain $R=10.55 \mathrm{~nm} \pm 0.06$ $\mathrm{nm}$ (red line). (b) Gold colloids measured at the cSAXS beamline (SLS) in a device with a COC window (green) and a Kapton window (blue). The form factor for solid spheres was fitted to the data and we obtain $R=16.13 \mathrm{~nm} \pm 0.03 \mathrm{~nm}$ (red line).

shown in Fig. $4 \mathrm{~b}$. In this case, we used colloids with a radius of $15 \mathrm{~nm}$. For these data, we were able to analyze the Guinier region at small $q$-values; we find a colloid radius of $17.88 \mathrm{~nm}$ $\pm 0.49 \mathrm{~nm}$. The pddf yields a radius of $16.50 \mathrm{~nm} \pm 0.04 \mathrm{~nm}$ and the direct fit $R=16.13 \mathrm{~nm} \pm 0.03 \mathrm{~nm}$ (red line). From these experiments, using a simple model system as scatterers, we conclude that the COC devices are well suited for performing SAXS experiments in microflow.

Experiments at synchrotron sources, i.e. with limited available time and highly complex setups, require robust, longlasting microfluidic devices. In our experience the COC devices are very reliable and outperform the UV-curable adhesive/Kapton devices. It is even possible to flush them by hand, i.e. at comparatively high pressure and little control, without breaking them. In combination with our sample holder (see Fig. S3 $\dagger$ ), the success rate for these devices is close to hundred percent. With respect to radiation damage, we observe a strong effect in the Kapton devices, which is, however, not due to the Kapton but to the UV-curable adhesive. In the COC devices the effect is much weaker. We will thus, in the future, investigate radiation resistance of the material in a more systematic way.

\section{Protein in flow}

In a next step, we use our COC devices for measurements on a more challenging system: the weakly scattering protein vimentin. Vimentin belongs to the family of intermediate filament (IF) proteins and is found in cells of mesenchymal origin. Together with actin filaments and microtubules, it forms a composite biopolymer network in the cytoplasm. In vitro, vimentin assembles from tetrameric subunits into extended filaments in a hierarchical manner upon the addition of $\mathrm{KCl} .{ }^{53}$ Within the first seconds, lateral assembly of the tetramers leads to so-called unit length filaments (ULFs), followed
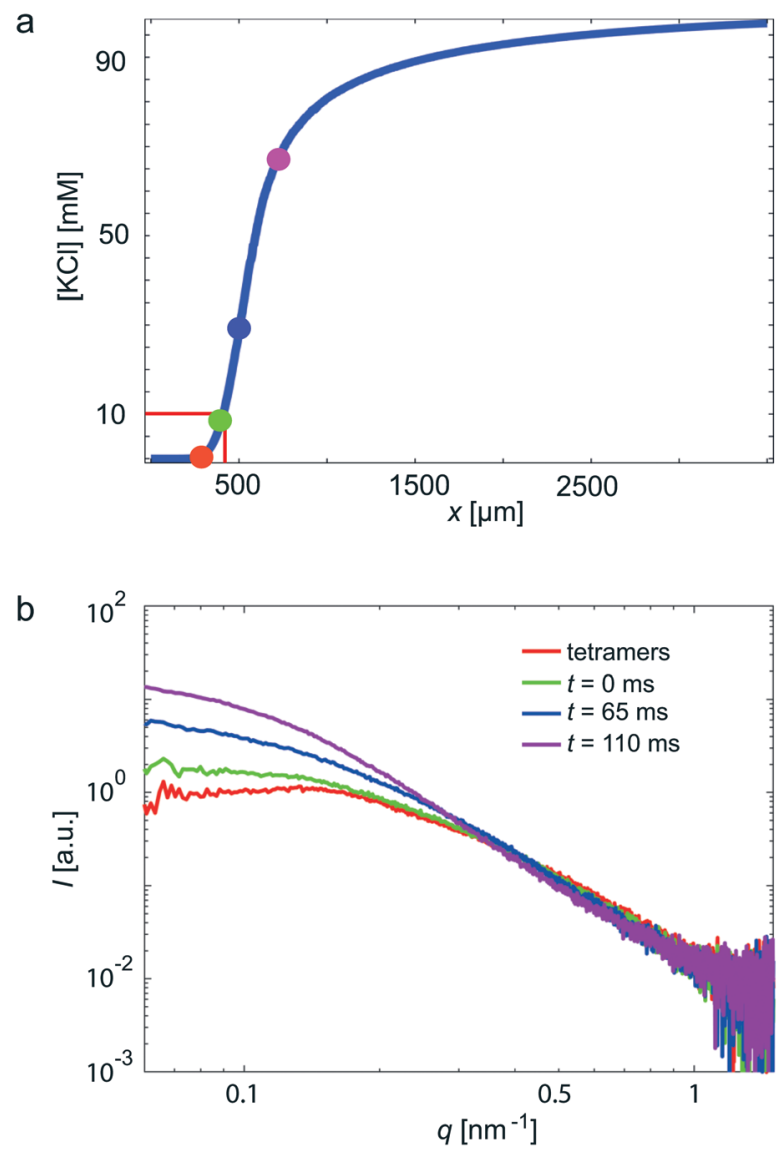

Fig. 5 Vimentin measurement in a COC device, performed at the cSAXS beamline, SLS. In total, four different positions (see Fig. 2a) in the scanned device are shown. (a) The $\mathrm{KCl}$ concentration along the central channel is shown. The four analyzed positions are indicated by colored circles. Assembly starts at a $\mathrm{KCl}$ concentration of $10 \mathrm{mM}$. (b) The red data curve corresponds to tetrameric vimentin; the green data curve is defined as the starting point of the assembly process $(t=0)$; blue and purple data curves refer to slightly later, yet early, time points. 
by an elongation mechanism, which may take minutes to hours and leads to micrometer long filaments. ${ }^{21,54,55}$ As a critical $\mathrm{KCl}$ concentration for assembly at these high protein concentrations, $10 \mathrm{mM}$ was previously identified. ${ }^{23}$ By contrast to previous experiments, ${ }^{21,23}$ we here realize fast mixing by strong hydrodynamic focusing of the central protein stream (see Fig. 3b). ${ }^{4,56}$ Thus, the width of the central stream is about $10 \mu \mathrm{m}$ and the diffusion time scale of the $\mathrm{KCl}$ into the central stream is on the order of $10 \mathrm{~ms}$. The rapid increase of the $\mathrm{KCl}$ concentration, as obtained from the FEM simulations, is presented in Fig. 5a. The color code of the individual data points corresponds to the positions in Fig. 2a and the data curves in Fig. 5b. The red curve shows the unassembled tetrameric state, whereas the green curve indicates the start of the assembly. We observe that the forward scattering $I_{0}$ increases with increasing assembly time, indicating an increase in molecular weight and, thus, assembly. Furthermore, the scattering curves steepen as the assembly proceeds. This change can be interpreted as an increase in thickness of the rod-shaped scatterers, corresponding to the known lateral assembly step of vimentin and in agreement with previously published work. ${ }^{21,55}$ Notably, we capture very early time points in this particular experiment.

\section{Conclusions}

To conclude, we present and characterize a microfluidic device fully made of COC. We demonstrate that the device is well-suited for X-ray measurements in flow. Weak SAXS signals from proteins, even of minute sample volumes, may be detected in a reliable, high-quality way. For benchmarking, we directly compare the performance of the COC devices to devices including Kapton windows. Aside from leading to SAXS data of equal quality, the COC devices provide a number of advantages over the UV-curable adhesive/Kapton devices. Above all, they are fabricated solely from one material, i.e. one type of COC without bonding layers, thus avoiding any gluing or interfaces, which are typically prone to leakage. Instead of plasticizing a thin layer on top of the COC sheet, we heat up the whole ensemble of two COC layers well above their glass transition temperature. As the unstructured sealing layer is much thinner $(20 \mu \mathrm{m})$ than the structured main layer $(240 \mu \mathrm{m})$ its viscosity decreases more rapidly, allowing for strong bonding. Indeed, the devices are more reliable than other devices we have tested here or in the past. Furthermore, COC materials have a high X-ray transmission and resist radiation. The device fabrication is straightforward and very reproducible at high quality, as it is already established for PDMS-glass devices. By contrast to commercial devices, as used for example in protein crystallography, the channel design is highly adaptable and flexible. Due to the employed lithography methods, the design of the microfluidic device can easily be customized. We believe that this approach will enable the community to perform various $\mathrm{X}$-ray/microfluidics experiments in a very reliable way. Additionally, we are able to show that even weakly scattering pro- tein signals may be investigated in this type of device. We thus expect a large number of different areas of application of these devices in the fields of biophysics, molecular biology and biochemistry.

\section{Conflicts of interest}

There are no conflicts of interest.

\section{Acknowledgements}

The authors thank Susanne Bauch for preparing the protein and TOPAS Advanced Polymers GmbH, Frankfurt, Germany, for providing the COC for the sealing layer. We furthermore thank Thomas Pfohl for helpful discussions. This work was financially supported by the Deutsche Forschungsgemeinschaft (DFG) in the framework of SFB 755, "Nanoscale Photonic Imaging", projects $\mathrm{B} 07$ and C10, and the Cluster of Excellence and DFG Research Center "Nanoscale Microscopy and Molecular Physiology of the Brain" (CNMPB).

\section{References}

1 D. C. Duffy, J. C. McDonald, O. J. A. Schueller and G. M. Whitesides, Anal. Chem., 1998, 70, 4974-4984.

2 A. Ghazal, J. P. Lafleur, K. Mortensen, J. P. Kutter, L. Arleth and G. V. Jensen, Lab Chip, 2016, 16, 4263-4295.

3 S. Köster and T. Pfohl, Mod. Phys. Lett. B, 2012, 26, 1230018.

4 L. Pollack, M. W. Tate, N. C. Darnton, J. B. Knight, S. M. Gruner, W. A. Eaton and R. H. Austin, Proc. Natl. Acad. Sci. U. S. A., 1999, 96, 10115-10117.

5 L. Pollack, M. W. Tate, A. C. Finnefrock, C. Kalidas, S. Trotter, N. C. Darnton, L. Lurio, R. H. Austin, C. A. Batt, S. M. Gruner and S. G. J. Mochrie, Phys. Rev. Lett., 2001, 86, $4962-4965$.

6 D. J. Segel, A. Bachmann, J. Hofrichter, K. O. Hodgson, S. Doniach and T. Kiefhaber, J. Mol. Biol., 1999, 288, 489-499.

7 S. Akiyama, S. Takahashi, T. Kimura, K. Ishimori, I. Morishima, Y. Nishikawa and T. Fujisawa, Proc. Natl. Acad. Sci. U. S. A., 2002, 99, 1329-1334.

8 T. Uzawa, S. Akiyama, T. Kimura, S. Takahashi, K. Ishimori, I. Morishima and T. Fujisawa, Proc. Natl. Acad. Sci. U. S. A., 2004, 101, 1171-1176.

9 T. Uzawa, T. Kimura, K. Ishimori, I. Morishima, T. Matsui, M. Ikeda-Saito, S. Takahashi, S. Akiyama and T. Fujisawa, J. Mol. Biol., 2006, 357, 997-1008.

10 M. Arai, E. Kondrashkina, C. Kayatekin, C. R. Matthews, M. Iwakura and O. Bilsel, J. Mol. Biol., 2007, 368, 219-229.

11 Y. Wu, E. Kondrashkina, C. Kayatekin, C. R. Matthews and O. Bilsel, Proc. Natl. Acad. Sci. U. S. A., 2008, 105, 13367-13372.

12 M. Arai, M. Iwakura, C. R. Matthews and O. Bilsel, J. Mol. Biol., 2011, 410, 329-342.

13 R. Graceffa, R. P. Nobrega, R. A. Barrea, S. V. Kathuria, S. Chakravarthy, O. Bilsel and T. C. Irving, J. Synchrotron Radiat., 2013, 20, 820-825.

14 R. Russell, I. S. Millett, M. W. Tate, L. W. Kwok, B. Nakatani, S. M. Gruner, S. G. J. Mochrie, V. Pande, S. Doniach, D. 
Herschlag and L. Pollack, Proc. Natl. Acad. Sci. U. S. A., 2002, 99, 4266-4271.

15 R. Das, L. W. Kwok, I. S. Millett, Y. Bai, T. T. Mills, J. Jacob, G. S. Maskel, S. Seifert, S. G. Mochrie, P. Thiyagarajan, S. Doniach, L. Pollack and D. Herschlag, J. Mol. Biol., 2003, 332, 311-319.

16 L. W. Kwok, I. Shcherbakova, J. S. Lamb, H. Y. Park, K. Andresen, H. Smith, M. Brenowitz and L. Pollack, J. Mol. Biol., 2006, 355, 282-293.

17 J. Lamb, L. Kwok, X. Qiu, K. Andresen, H. Y. Park and L. Pollack, J. Appl. Crystallogr., 2008, 41, 1046-1052.

18 J. C. Schlatterer, L. W. Kwok, J. S. Lamb, H. Y. Park, K. Andresen, M. Brenowitz and L. Pollack, J. Mol. Biol., 2008, 379, 859-870.

19 R. Dootz, A. Otten, S. Köster, B. Struth and T. Pfohl, J. Phys.: Condens. Matter, 2006, 18, 639-652.

20 T. Pfohl, A. Otten, S. Köster, R. Dootz, B. Struth and H. M. Evans, Biomacromolecules, 2007, 8, 2167-2172.

21 M. E. Brennich, J.-F. Nolting, C. Dammann, B. Nöding, S. Bauch, H. Herrmann, T. Pfohl and S. Köster, Lab Chip, 2011, 11, 708-716.

22 S. Köster, H. M. Evans, J. Y. Wong and T. Pfohl, Biomacromolecules, 2008, 9, 199-207.

23 O. Saldanha, M. E. Brennich, M. Burghammer, H. Herrmann and S. Köster, Biomicrofluidics, 2016, 10, 24108.

24 O. Saldanha, R. Graceffa, C. Y. J. Hémonnot, C. Ranke, G. Brehm, M. Liebi, B. Marmiroli, B. Weinhausen, M. Burghammer and S. Köster, ChemPhysChem, 2017, 18, 1220-1223.

25 R. Stehle, G. Goerigk, D. Wallacher, M. Ballauff and S. Seiffert, Lab Chip, 2013, 13, 1529-1537.

26 R. Dootz, H. Evans, S. Köster and T. Pfohl, Small, 2007, 3, 96-100.

27 M. Trebbin, D. Steinhauser, J. Perlich, A. Buffet, S. V. Roth, W. Zimmermann, J. Thiele and S. Förster, Proc. Natl. Acad. Sci. U. S. A., 2013, 110, 6706-6711.

28 H. Evans, R. Dootz, S. Köster and T. Pfohl, Bull. Pol. Acad. Sci.: Tech. Sci., 2007, 55, 217-227.

29 B. Weinhausen and S. Köster, Lab Chip, 2013, 13, 212-215.

30 J. Steigert, S. Haeberle, T. Brenner, C. Müller, C. P. Steinert, P. Koltay, N. Gottschlich, H. Reinecke, J. Rühe, R. Zengerle and J. Ducrée, J. Micromech. Microeng., 2007, 17, 333-341.

31 C. P. Steinert, J. Mueller-Diekmann, M. Weiss, M. Roessle, R. Zengerle and P. Koltay, 2007, IEEE 20th International Conference on Micro Electro Mechanical Systems (MEMS), 2007, pp. 561-564.

32 J. D. Ng, P. J. Clark, R. C. Stevens and P. Kuhn, Acta Crystallogr., Sect. D: Biol. Crystallogr., 2008, 64, 189-197.

33 S. Guha, S. L. Perry, A. S. Pawate and P. J. Kenis, Sens. Actuators, B, 2012, 174, 1-9.

34 M. Maeki, A. S. Pawate, K. Yamashita, M. Kawamoto, M. Tokeshi, P. J. A. Kenis and M. Miyazaki, Anal. Chem., 2015, 87, 4194-4200.

35 A. S. Pawate, V. Šrajer, J. Schieferstein, S. Guha, R. Henning, I. Kosheleva, M. Schmidt, Z. Ren, P. J. A. Kenis and S. L.
Perry, Acta Crystallogr., Sect. F: Struct. Biol. Commun., 2015, 71, 823-830.

36 B. F. B. Silva, M. Zepeda-Rosales, N. Venkateswaran, B. J. Fletcher, L. G. Carter, T. Matsui, T. M. Weiss, J. Han, Y. Li, U. Olsson and C. R. Safinya, Langmuir, 2015, 31, 4361-4371.

37 S. A. Aghvami, A. Opathalage, Z. Zhang, M. Ludwig, M. Heymann, M. Norton, N. Wilkins and S. Fraden, Sens. Actuators, B, 2017, 247, 940-949.

38 T. I. Wallow, A. M. Morales, B. Simmons, M. C. Hunter, K. Lee Krafcik, L. A. Domeier, S. Sickafoose, K. Patel and A. Gardea, Lab Chip, 2008, 7, 1825-1831.

39 F. I. Uba, B. Hu, K. Weerakoon-Ratnayake, N. Oliver-Calixte and S. A. Soper, Lab Chip, 2015, 15, 1038-1049.

40 H. Cherdron, M.-J. Brekner and F. Osan, Angew. Makromol. Chem., 1994, 223, 121-133.

41 S. R. Quake and A. Scherer, Science, 2000, 290, 1536-1540.

42 H. Y. Park, X. Qiu, E. Rhoades, J. Korlach, L. W. Kwok, W. R. Zipfel, W. W. Webb and L. Pollack, Anal. Chem., 2006, 78, 4465-4473.

43 R. B. Urbani, Ph.D. thesis, Universität Basel, 2015.

$44 \mathrm{H}$. Herrmann, L. Kreplak and U. Aebi, in Intermediate Filament Cytoskeleton, ed. M. B. Omary and P. A. Coulombe, Academic Press, 2004, ch. Method. Cell Biol., vol. 78, pp. 3-24.

45 B. Nöding and S. Köster, Phys. Rev. Lett., 2012, 108, 088101.

46 S. Winheim, A. R. Hieb, M. Silbermann, E.-M. Surmann, T. Wedig, H. Herrmann, J. Langowski and N. Mücke, PLoS One, 2011, 6, 1-7.

47 R. Dinapoli, A. Bergamaschi, B. Henrich, R. Horisberger, I. Johnson, A. Mozzanica, E. Schmid, B. Schmitt, A. Schreiber, X. Shi and G. Theidel, Nucl. Instrum. Methods Phys. Res., Sect. A, 2011, 650, 79-83.

48 B. Henrich, A. Bergamaschi, C. Broennimann, R. Dinapoli, E. Eikenberry, I. Johnson, M. Kobas, P. Kraft, A. Mozzanica and B. Schmitt, Nucl. Instrum. Methods Phys. Res., Sect. A, 2009, 607, 247-249.

49 P. V. Konarev, V. V. Volkov, A. V. Sokolova, M. H. J. Koch and D. I. Svergun, J. Appl. Crystallogr., 2003, 36, 1277-1282.

50 H. Herrmann, M. Häner, M. Brettel, S. A. Müller, K. N. Goldie, B. Fedtke, A. Lustig, W. W. Franke and U. Aebi, J. Mol. Biol., 1996, 264, 933-953.

51 I. Hofmann, H. Herrmann and W. W. Franke, Eur. J. Cell Biol., 1991, 56, 328-341.

52 R. Barrett, M. Faucon, J. Lopez, G. Cristobal, F. Destremaut, A. Dodge, P. Guillot, P. Laval, C. Masselon and J.-B. Salmon, Lab Chip, 2006, 6, 494-499.

53 N. Mücke, T. Wedig, A. Bürer, L. N. Marekov, P. M. Steinert, J. Langowski, U. Aebi and H. Herrmann, J. Mol. Biol., 2004, 340, 97-114.

54 C. G. Lopez, O. Saldanha, K. Huber and S. Köster, Proc. Natl. Acad. Sci. U. S. A., 2016, 113, 11152-11157.

55 H. Herrmann, H. Bär, L. Kreplak, S. V. Strelkov and U. Aebi, Nat. Rev. Mol. Cell Biol., 2007, 8, 562-573.

56 J. B. Knight, A. Vishwanath, J. P. Brody and R. H. Austin, Phys. Rev. Lett., 1998, 80, 3863-3866. 


\section{Supplementary Information (SI)}

\section{Cyclic olefin copolymer as an X-Ray compatible material for microfluidic devices}

Manuela Denz, ${ }^{a \ddagger}$ Gerrit Brehm, $,{ }^{a}, b$ Clément Y. J. Hémonnot, ${ }^{a}$ Heidi Spears, ${ }^{a}$ Andrew Wittmeier, ${ }^{a}$ Chiara Cassini, ${ }^{a}$ Oliva Saldanha, ${ }^{a}$ Eleonora Perego, ${ }^{a}$ Ana Diaz, ${ }^{c}$ Manfred Burghammer ${ }^{d}$ and Sarah Köster ${ }^{a, b *}$

\section{Device Fabrication (Figure S1)}

In this section, we present more details on our device fabrication method. Fig. S1 (following page) is an extension of Fig. 1 in the main text. All steps, starting from the silicon-wafer with channel structure all the way to the finished device, are shown.

Figure S1 Device Fabrication steps (see following page) (a) The channel structures (black) are defined by photolithography using SU8-2150 negative photo resist spin coated on Si-wafers to a final height of $160 \mu \mathrm{m}$. (b) PDMS copies (blue) of the channel structures are produced. PDMS and cross-linker are mixed at a ratio of 10:1 and poured onto the structured Si-wafers. After evacuating the PDMS for 20 min to remove all air bubbles, it is cured by backing for $1 \mathrm{~h}$ at $65^{\circ} \mathrm{C}$. (c) A $1 \mathrm{~mm}$ thick glass slide (gray) is prepared with UV-curable adhesive (yellow) and all air bubbles are removed with a needle. (d) The PDMS stamp is pressed into the adhesive and cured with UV-light $(\lambda=366 \mathrm{~nm}$ ) for three minutes. (e) After detaching the PDMS stamp from the glass slide and removing residual structures with a scalpel, the UV-curable adhesive is cured again for three minutes. (f) The stamp (yellow) and a COC sheet (green) are sandwiched between two sheets of paper, followed by two sheets of aluminum foil. This assembly is heated above the glass transition temperature of the $\mathrm{COC}\left(130{ }^{\circ} \mathrm{C}\right)$ for 5 minutes, with the help of a hot press. It is then pressed with initially $2.2 \mathrm{kN}$ for 10 minutes. Thus, the structured UV-cured adhesive is transferred into the COC layer. $(\mathrm{g}$ ) After cooling down the hot press, the stamp is removed from the COC after wetting with 2-propanol, which flows between the two layers because of capillary forces. The adhesive stamp can be reused. (h) Holes for the inlet and outlet are punched with a biopsy puncher (diameter $0.5 \mathrm{~mm}$ ). (i) A $20 \mu \mathrm{m}$ thick COC sealing layer and the channel structure in COC are sandwiched in paper and placed in a lamination machine with heat and pressure control. The device is sealed at a temperature of $100{ }^{\circ} \mathrm{C}$ and a speed of $7.6 \times 10^{-3} \mathrm{~m} / \mathrm{s}$. (j) The finished COC device contains channels with a width of $200 \mu \mathrm{m}$, a height of $160 \mu \mathrm{m}$ and a total thickness of $70 \mu \mathrm{m}$ COC within the beam path.

\footnotetext{
$\ddagger$ These authors contributed equally to this work

${ }^{a}$ Institute for X-Ray Physics, University of Goettingen, 37077 Göttingen

${ }^{b}$ Center Nanoscale Microscopy and Molecular Physiology of the Brain (CNMPB), Göttingen, Germany

${ }^{c}$ Swiss Light Source, Paul Scherrer Institut, 5232 Villigen, Switzerland

${ }^{d}$ European Synchrotron Radiation Facility, 38043 Grenoble, France.

* E-Mail: sarah.koester@phys.uni-goettingen.de
} 
a
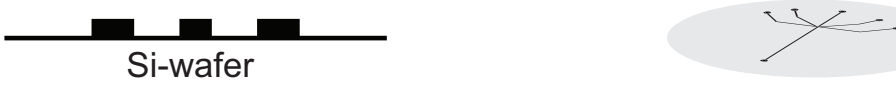

Si-wafer

b

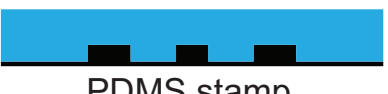

PDMS stamp

UV-curable adhesive

d
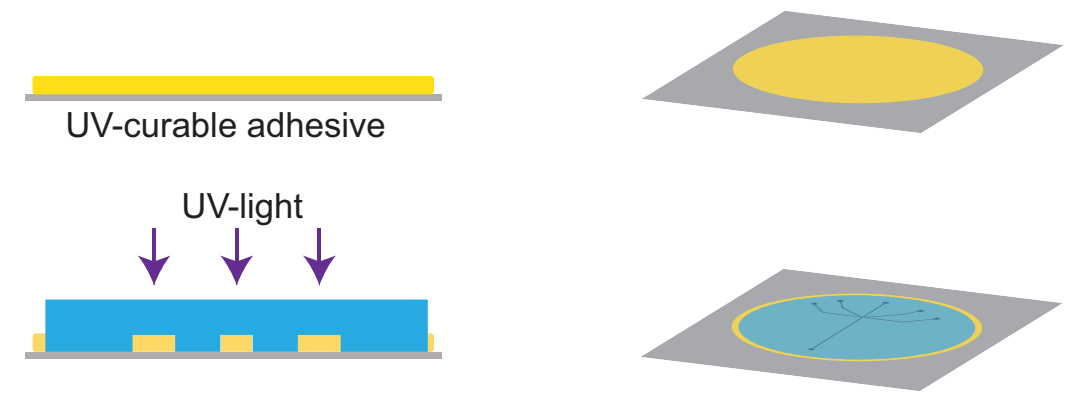

e
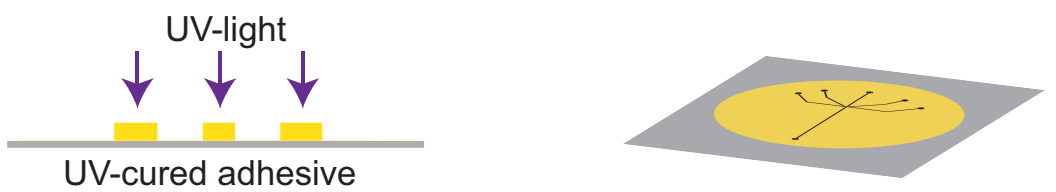

f

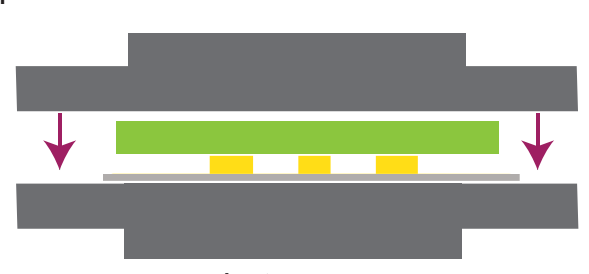

hot press

g

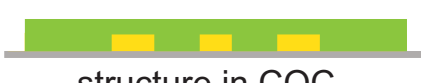

structure in $\mathrm{COC}$

h
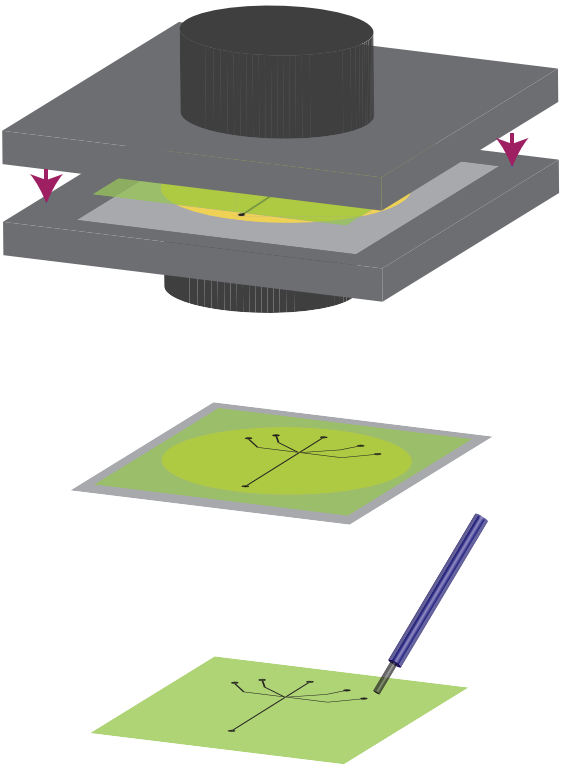

i

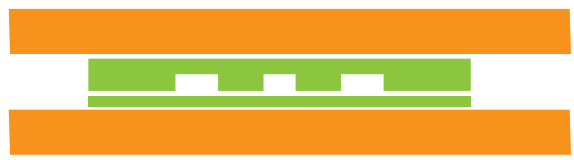

lamination machine

j

finished device

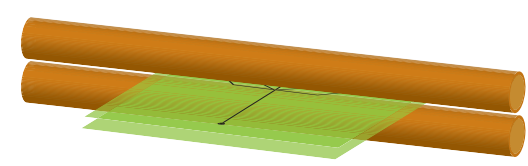




\section{Cross Section of the Channel Structure (Figure S2)}

The cross sectional view of a typical device is depicted in Figure S2. Due to the challenge in cutting the device precisely without squeezing the channels the edges appear blurry and the bottom of the device is slightly stretched. However, the well-defined channel structure with straight walls is clearly visible.

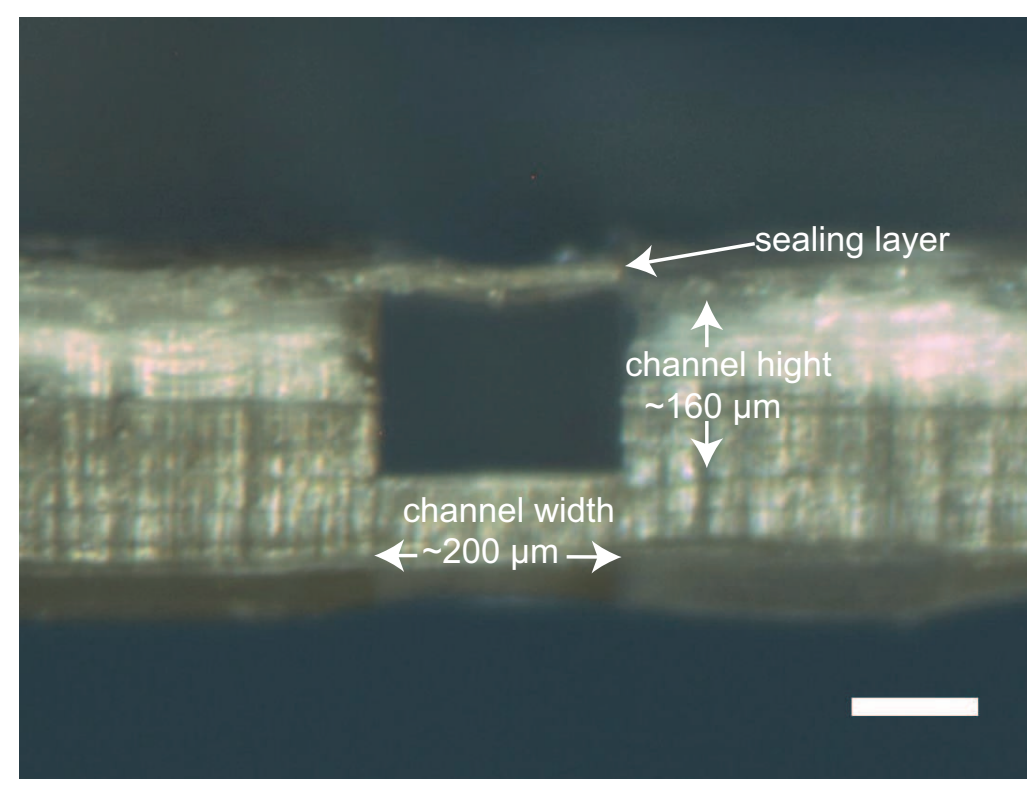

Figure S2 Side view of a typical COC device. The channel height is $\sim 160 \mu \mathrm{m}$ and the channel width $\sim 200 \mu \mathrm{m}$. The scale bar corresponds to $100 \mu \mathrm{m}$. 


\section{Sample Holder (Figure S3)}

In the following the used sample holder is described and shown in detail.

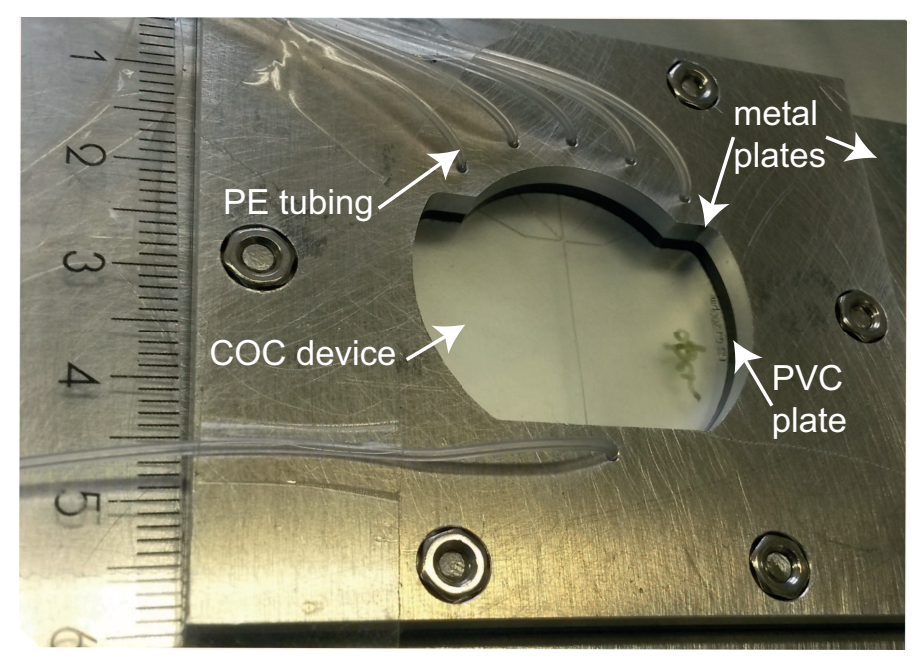

Figure S3 The sample holder consists of two metal plates with different sizes. The metal back plate is larger to allow for mounting to the setup. The upper metal plate contains openings for the tubing. Both plates contain an opening to perform measurements inside the COC device. Sandwiched between the upper metal plate and the COC device is a PVC plate that contains holes for the tubing and indentations for o-rings. The o-rings get compressed by tightening six screws and therefore seal the connection between the device and the tubing. The scale shown is in $\mathrm{cm}$. 


\subsection{Microfluidic Step-Devices for X-Ray Measurements}

The microfluidic COC step-device employed in this work fulfils different rolls in the time-resolved SAXS measurements. Note that this Sec. focuses on COC stepdevices. Non-step devices are presented and discussed in Sec. 5.1. The mixing process inside the device and the time resolution achieved in time-resolved SAXS measurements are simulated with FEM simulations using COMSOL Multiphysics ${ }^{\circledR}$ v. 5.3 software (COMSOL AB, Stockholm, Sweden), hereinafter COMSOL. Varying the meshsize of the FEM simulation influences the obtained results. This chapter investigates this effect using the results of the time-resolved scanning SAXS measurements of vimentin assembly measured at the cSAXS beamline (Synchroton Lichtquelle Schweiz (SLS), Paul Scherrer Institut (PSI), Villigen, Switzerland). Furthermore, tools developed for data analysis are presented.

\subsubsection{Finite Element Method Simulations}

In Sec. 3.6 the principle of FEM is introduced by approximating an unknown solution with smaller and solvable sections. In this work, COMSOL is used to simulate the central part of the microfluidic device (see Fig. 5.1), where the vimentin assembly kinetics occur. A mesh of tetrahedral elements is used to approximate the central section of the device to numerically solve the Navier-Stokes equation with no-slip boundary condition and the transport equations. To reduce

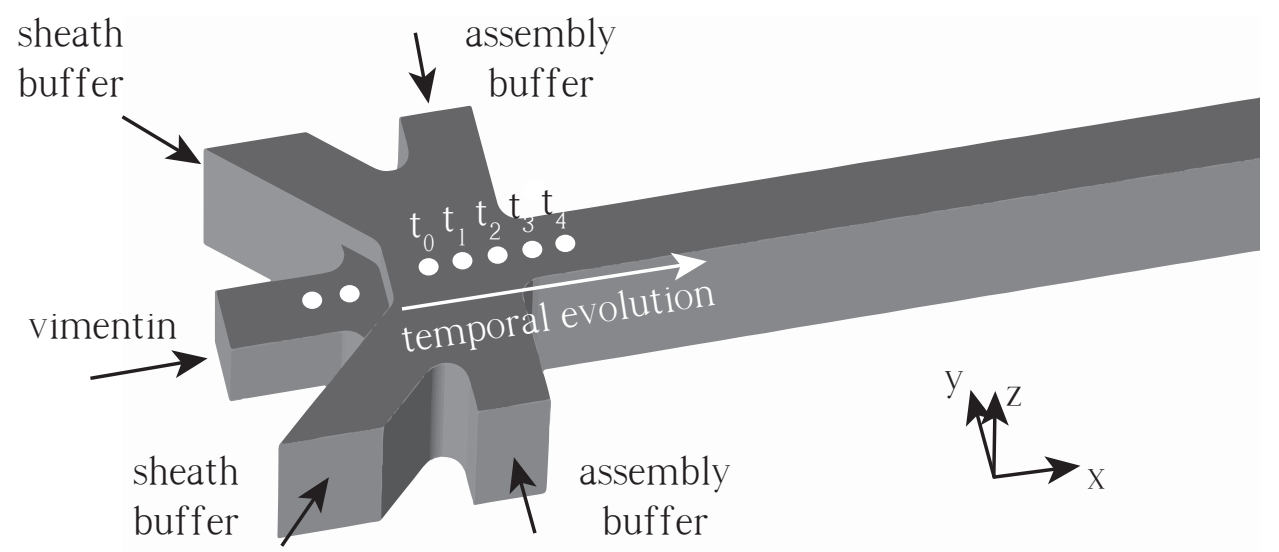

Figure 5.1.: Model of the central mixing region of the device used for FEM simulations. The channel width is $200 \mu \mathrm{m}$ and the channel height is $440 \mu \mathrm{m}$ the step height is $240 \mu \mathrm{m}$. The kinetics of the vimentin assembly is observed with scanning-SAXS, indicated by the white dots. 


\section{\begin{tabular}{l|l} 
Chapter 5 & RESULTS
\end{tabular}}

computational time, occurring symmetry in the $x-y$ and $x-z$ plane of the device is exploited. Imposing symmetry conditions on the symmetry planes ensures that the reduced simulated volume adequately represents the entire section. Regions with few expected dynamics contain larger tetrahedral mesh elements, reducing the computational time further. These regions are shown in grey in Fig. 5.2a. Towards the central part of the device, shown in dark blue in Fig. 5.2a, smaller mesh elements are used, as here the relevant mixing process occurs. A region with intermediate sized tetrahedrons, shown in light blue in Fig. 5.2a, connects both regions to reduce artifacts in the simulation. Meshes with different sized tetrahedral elements for each region are shown Fig. 5.2b-d. An overview of the a side length of the mesh elements is given in Tab. 5.1.

Figure $5.2 \mathrm{~b}$ consist of the smallest mesh elements. The blue region contains tetrahedrons with a side length between $2 \mu \mathrm{m}$ and $3 \mu \mathrm{m}$, whereas in Fig. 5.2c+d this region contains tetrahedrons with a side length between $4 \mu \mathrm{m}$ to $6 \mu \mathrm{m}$ and $6 \mu \mathrm{m}$ to $9 \mu \mathrm{m}$, respectively. The intermediate mesh size region, shown in light blue in Fig. 5.2a, contains tetrahedrons with a side length between $4 \mu \mathrm{m}$ to $5 \mu \mathrm{m}$ for Fig. $5.2 \mathrm{~b}$ and $8 \mu \mathrm{m}$ to $10 \mu \mathrm{m}$ and $12 \mu \mathrm{m}$ to $15 \mu \mathrm{m}$ for Fig. 5.2c and Fig. $5.2 \mathrm{~d}$, respectively. The outer light grey region in Fig. 5.2a with few dynamics,

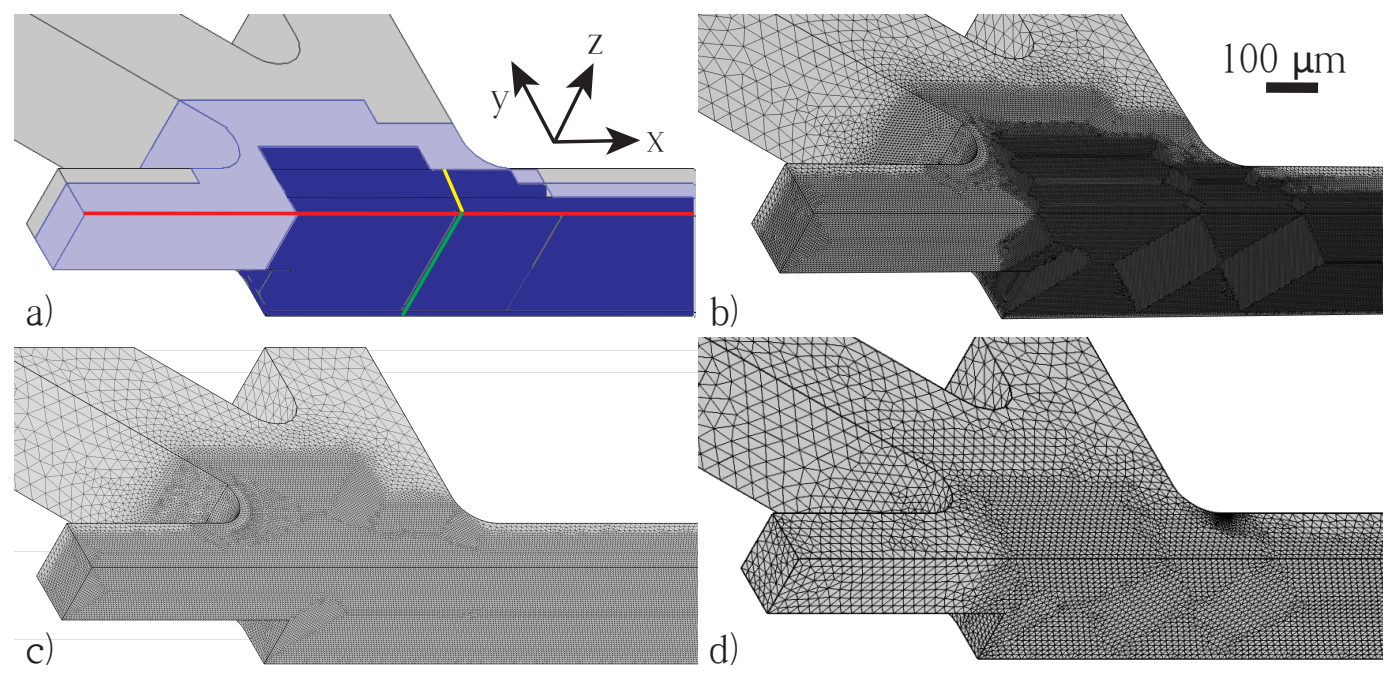

Figure 5.2.: Mesh creation for FEM simulations. The employed geometry is subdivided in three regions, indicated by different colours in a). The red, green and yellow lines represent the plot axis for the vimentin and $\mathrm{KCl}$ concentrations in Fig. 5.3. The meshsize for each region is individually adapted and increases from the inner part (dark blue) to the outer part (grey) of the geometry. This in visualized by an increase in the side length of the tetrahedral structures. b)-d) Show calculated meshes, with increasing meshsizes for each region from b) to d). 
Table 5.1.: Minimum and maximum tetrahedral element sizes for the different mesh regions for each set of meshsizes containing the most, intermediate and least element numbers shown in Fig. 5.2b, Fig. 5.2c and Fig. 5.2d, respectively.

mesh element side length for the sub-regions shown in Fig. 5.2a inner (dark blue) middle (light blue) outer (grey)

Fig. $5.2 \mathrm{~b}$

Fig. $5.2 \mathrm{c}$

Fig. 5.2d

\begin{tabular}{|c|c|c|}
\hline 2 to $3 \mu \mathrm{m}$ & 4 to $5 \mu \mathrm{m}$ & 9 to $30 \mu \mathrm{m}$ \\
\hline 4 to $6 \mu \mathrm{m}$ & 8 to $10 \mu \mathrm{m}$ & 9 to $30 \mu \mathrm{m}$ \\
\hline 6 to $9 \mu \mathrm{m}$ & 12 to $15 \mu \mathrm{m}$ & 9 to $30 \mu \mathrm{m}$ \\
\hline
\end{tabular}

contains for all three shown meshes tetrahedrons with a side length between $9 \mu \mathrm{m}$ to $30 \mu \mathrm{m}$. The impact of different meshsizes on the simulation result is addressed in Sec. 5.2.1.1.

The simulations for the geometry of the microfluidic device are performed using an upper limit diffusion coefficient of $D_{\text {vim }}=2.1^{-11} \mathrm{~m}^{2} / \mathrm{s}$ and $D_{\mathrm{KCl}}=1.84^{-9} \mathrm{~m}^{2} / \mathrm{s}$ for vimentin and $\mathrm{KCl}$, respectively. The device is simulated with water as the transporting medium, as an approximation for the aqueous buffers containing the vimentin and the $\mathrm{KCl}$ in the experiment. The concentrations $c$ represent the experimental conditions $\left(c_{\mathrm{vim}}=4 \mathrm{~g} / \mathrm{L}, c_{\mathrm{KCl}}=100 \mathrm{mM}\right)$. The flow rate for each assembly buffer inlet during the experiment is $100 \mu \mathrm{L} / \mathrm{h}$, whereas both sheath buffer inlets operate at $5 \mu \mathrm{L} / \mathrm{h}$, after all flow connections are established. The central vimentin inlet operates at a flow rate of $10 \mu \mathrm{L} / \mathrm{h}$. All flow rates in the simulation are adapted according to the reduced geometry originating from the use of symmetry.

\subsubsection{Mesh Size Dependency}

The size and number of mesh elements has a major impact on the computational time and the result of the simulation. Simulations are performed on a quarter of the mixing region subdivided in regions containing different sized tetrahedral mesh elements (see Fig. 5.2 and Tab. 5.1). The size and shape of each mesh region is adapted to the dynamics occurring at the respective position. Only a part of the simulated device is shown in Fig. 5.2, as the simulated length in the x-direction is $5 \mathrm{~mm}$.

For each set of meshsizes shown in Fig. 5.2b-d the calculated concentration for vimentin and $\mathrm{KCl}$ along the center of the device in flow direction $\mathrm{x}$, indicated by red line in Fig. 5.2a, is shown in Fig. 5.3a+b, respectively. For $\mathrm{KCl}$, no difference in concentration is observed between the different meshes, whereas the concentration 


\section{\begin{tabular}{l|l} 
Chapter 5 & RESULTS
\end{tabular}}

a)

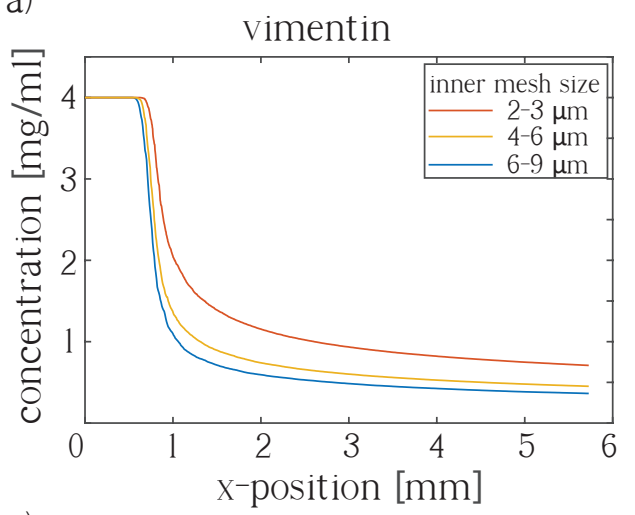

c)

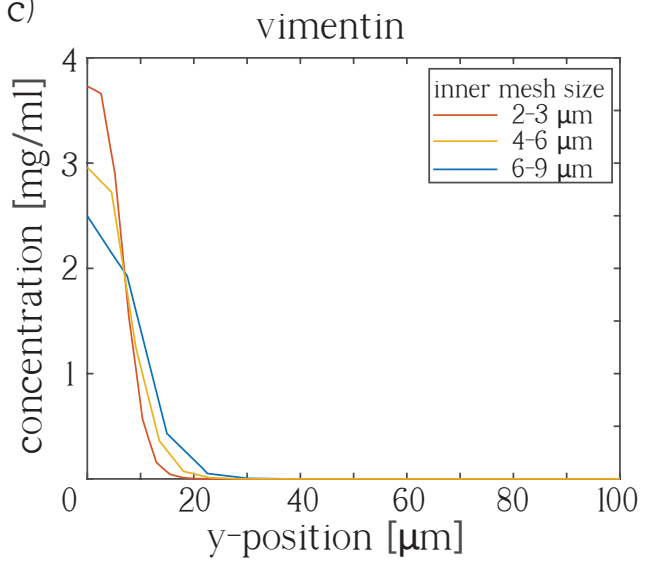

b)

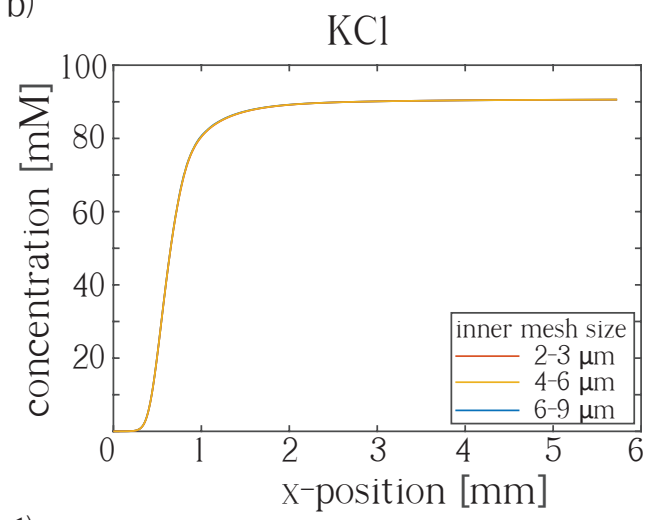

d)

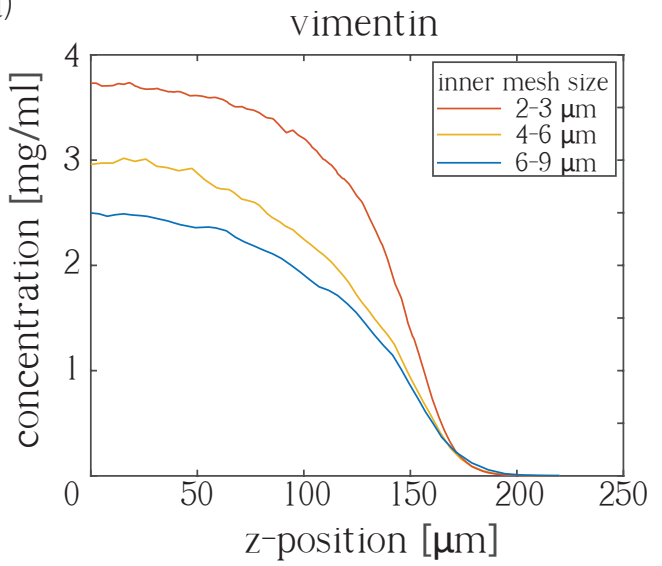

Figure 5.3.: Vimentin and $\mathrm{KCl}$ concentrations for different meshsizes. a) Vimentin concentration and b) $\mathrm{KCl}$ concentration along the center of the device in flow direction $\times$ (see red line in Fig. 5.2a. c) shows the vimentin concentration y-profile in $300 \mu \mathrm{m}$ distance downstream from the step (see yellow line in Fig. 5.2a, whereas d) shows the corresponding z-profile (see green line in Fig. 5.2a). Note that for c) and d) half profiles of the channel are shown and the 0 position represents the intersection of the yellow and green line with the red line Fig. 5.2a, thus the center of the device. 
in the center of the device for vimentin decreases faster for larger meshsizes. This observation is confirmed by the horizontal and vertical flow profiles of vimentin perpendicular to the flow direction, see Fig. $5.3 c+d$, respectively. The horizontal profile coordinates are indicated by the yellow line in Fig. 5.2a and only $100 \mu \mathrm{m}$ from the center of the device (red line in Fig. 5.2a) are shown, as no vimentin is contained in the remaining part. The vertical profile coordinates are indicated by the green line in Fig. 5.2a. Note that for both half profiles the 0 position represents the intersection of respective profile with the center of the device.

To compare the simulation results with each other the width of the hydrodynamically focused vimentin in the center of the device is estimated based on theoretical considerations. The volume percentage originating from the inflow of the central vimentin containing inlet is, in respect to the overall injected volume from the 5 inlets $\approx 5(10 \mu \mathrm{L} /(100+100+5+5+10) \mu \mathrm{L})$. Assuming the hydrodynamic focusing is nearly completed, the channel width of $200 \mu \mathrm{m}$ after the mixing region is applied in the following, resulting in $5 \%$ to be $10 \mu \mathrm{m}$. As the shown profiles represent half profiles, the vimentin should be focused to the first $5 \mu \mathrm{m}$ of the profiles. Based on the employed step geometry $\approx 2 / 3$ of the vertical profile contain vimentin, shown in Fig. 5.3d. Thus, the section filled by vimentin in the horizontal profile needs to be extended and is estimated to be $\approx 10 \%$, as the focusing is only nearly complete.

For the smallest meshsize containing the most mesh elements, shown in red in Fig. 5.3c, the half FWHM corresponds with $10 \mu \mathrm{m}$ to the made assumption, whereas for fewer mesh elements the concentration in the horizontal profile is broadened. In addition, the remaining maximum concentration is reduced. As all simulations parameters are kept constant in between the different simulations, this effect is based on the chosen meshsize in the different mesh regions. The results with the smallest network elements seem to describe the simulated system best in accordance with the theoretical expectations (see Sec. 3.6).

\subsubsection{Flow Profiles and Accessible Assembly Time Points}

Based on the FEM simulations both the flow rates and the geometry of the device are adjusted for experimental needs. Calculated concentration profiles for vimentin and $\mathrm{KCl}$ used for time-resolved SAXS measurements are shown in Fig. 5.4. The upper half of each subfigure depicts the $\mathrm{KCl}$ and the lower half the vimentin concentration. The center of the device is indicated by the white gap. Note, that in Fig. 5.4a neither the sheath buffer inlet nor the assembly buffer inlet are 


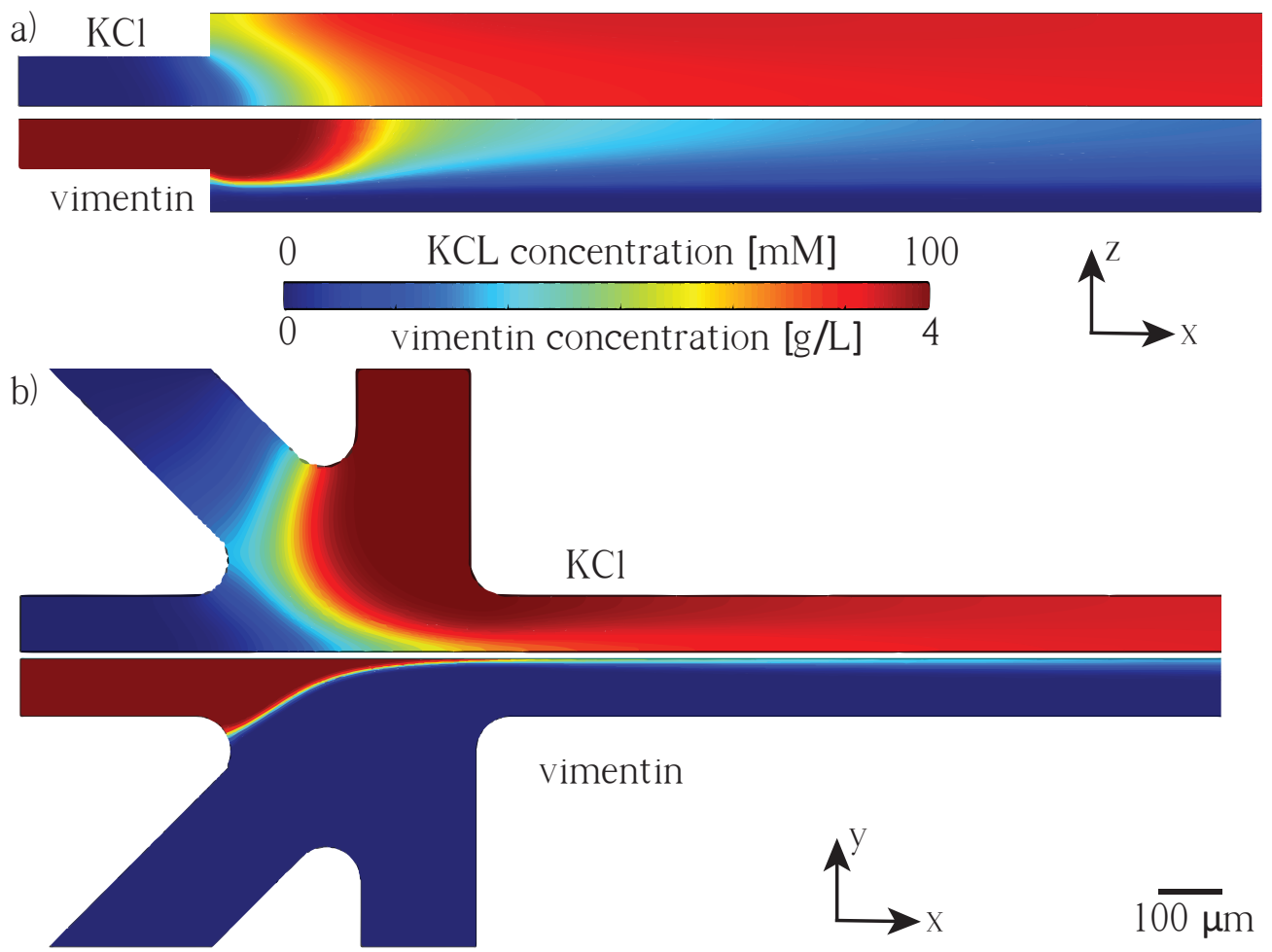

Figure 5.4.: Exemplary results of FEM simulations for the mesh displayed in Fig. $5.2 \mathrm{c}$ ). $\mathrm{KCl}$ and vimentin concentrations are show separately for the side view a) and the top view b). High concentrations in both cases are indicated by the red colour. The center of the device is indicated by the white gap between both halves.

visible, as the concentration profile is at the $y=0$ mark. Hydrodynamic focusing of the vimentin is visible in both directions perpendicular to the flow direction. The velocity profile along the center is calculated and displayed in blue in Fig. 5.5b. The kinks at $\mathrm{x}=0.5$ and $\mathrm{x}=5.5 \mathrm{~mm}$ are numerical artifacts from the simulation. The $\mathrm{KCl}$ threshold to start the vimentin assembly process is set to $10 \mathrm{mM} \mathrm{KCl}$, as suggested by Brennich et al. [24]. The first point in the $\mathrm{KCl}$ concentration plot (see Fig. $5.3 \mathrm{~b}$ ) above this threshold is traced, and the start of the vimentin assembly is correlated to the spatial coordinates of the device. Based on this starting point the reaction time is expressed by using the calculated velocity and the known element size and displayed in red in the same graph. This correlates to following the individual flow lines and to sum up the respective times. In the simulated section, corresponding to $6 \mathrm{~mm}$ around the mixing region, the first $\approx 5 \mathrm{~s}$ of vimentin assembly can be observed.

Correlation of spatial coordinates measured and the corresponding reaction 

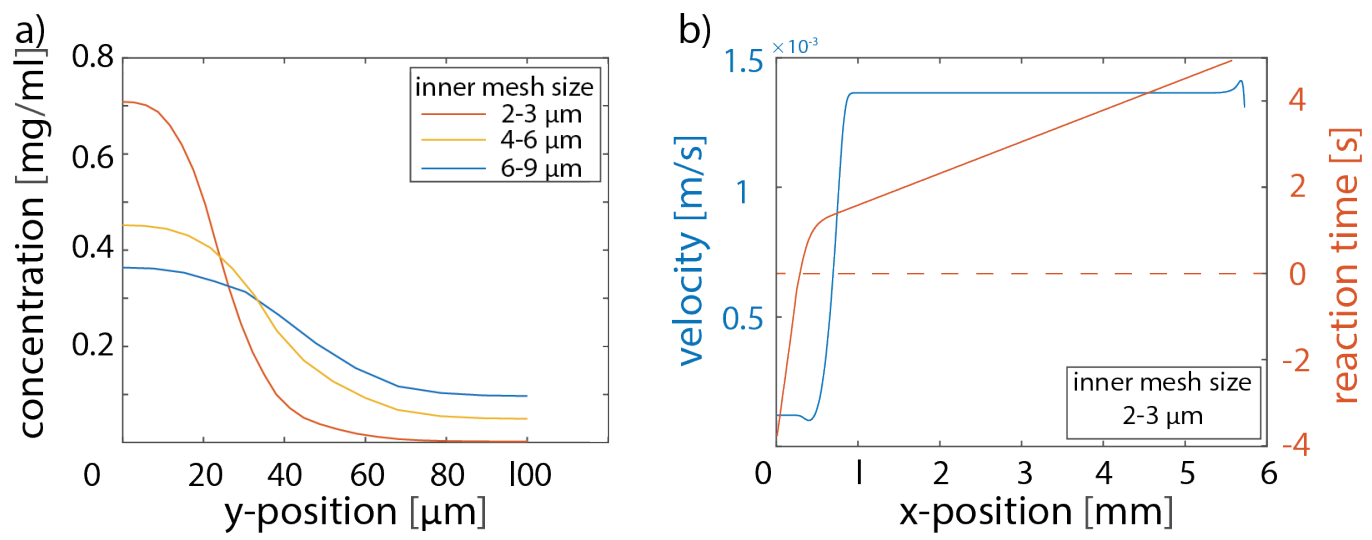

Figure 5.5.: a) Horizontal vimentin concentration profile at the end of the simulated device $(x=$ $5.5 \mathrm{~mm}$ ). The center of the device is represented by the $0 \mathrm{~mm}$ y-position, as only half of the profile is shown. Note, that only for the mesh containing the smallest elements the vimentin concentration at the channel walls $(y=100)$ is 0. b) Velocity along the center of the device in $\mathrm{m} / \mathrm{s}$ is shown in blue. Based on the $\mathrm{KCl}$ concentration threshold starting the vimentin assembly, the calculated velocity and the mesh size elements the reaction time corresponding to each lateral position is calculated and shown in red.

time are performed for the experimental data described in Sec. 5.2.4. Fig. 5.5a shows, in analogy to Fig. 5.3c, the horizontal concentration profile for vimentin. The $\mathrm{x}$-coordinate corresponds to the last simulated position. The time needed for a molecule to be transported convectively between the x-position of the first horizontal profile and the last simulated x-position is calculated with the know velocity profile shown in Fig. $5.5 \mathrm{~b}$ and is $\approx 5 \mathrm{~s}$. To estimate the theoretical broadening of the vimentin distribution during this time the square root of the mean square displacement in one dimension is employed.

$$
x=\sqrt{D t}
$$

with $D$ the diffusion constant for $\mathrm{KCl}=2.110^{-11} \mathrm{~m}^{2} / \mathrm{s}$. With an elapsed time of $\approx 5 \mathrm{~s}$ the expected broadening is around $10 \mu \mathrm{m}$. This fits well with the simulated horizontal vimentin distribution along which shows an increase of $\approx 15 \mu \mathrm{m}$ in width.

The device geometry and flow rates employed enable the hydrodynamic focusing in both the horizontal and the vertical axis (see Fig. 5.4), as well as fast mixing times (see Fig. 5.5). Whereas for each measuring position, indicated by the white dots in Fig. 5.1, the assembly time can now be given (see Fig. 5.5), the recorded signal to noise ratio for SAXS differs between measuring points. This variation is 


\section{\begin{tabular}{l|l} 
Chapter 5 & RESULTS
\end{tabular}}

inherent to a step device and is addressed in the following.

\subsubsection{Correction Factor Calculation}

Depending on the position inside the microfluidic device, incident $X$-rays pass through different ratios of COC, buffer and sample. Whereas the absorption coefficient for buffer solution and sample solution is similar (see Sec. 4.3.4), one material property of the employed device material COC is lower photon absorbance than aqueous solution for the photon energies used in this experiment. Assuming the device height is constant, outside the channel the photons pass only through COC and thus the number of transmitted photons is higher compared to a data point acquired inside the channel. Depending on the geometry of the device, a change in the transmission factor inside the channel occurs. This change is a result of the COC step, which changes the ratio of aqueous solution and COC along the central flow lines (see white dots in Fig. 5.1). In the experiment performed at cSAXS the transmitted intensity is measured by a diode on the beamstop which is placed in front of the detector. The number of incident photon per seconds, hereinafter referred to as 'flux', are monitored prior to sample interaction for each measurement taken and are shown in Fig. 5.6. Each data point is measured twice, once for the background measurement shown in red and subsequently for the identical position with vimentin shown in blue. Note, that the entire scanning region is measured prior to changing the buffer solution to vimentin in the central inlet for the second exposure. The change of solutions is performed without physical detachment of the sample holder containing the device to the motor stage responsible for the sample movement resulting in the identical position for both data points. The transmitted intensity of the vimentin is normalized by subtraction of the transmitted intensity of the background in a point-wise manner. The recorded flux of 11 subsequent data points along the center of device is shown in Fig. 5.6a.

The distance between each point is $25 \mu \mathrm{m}$ in the flow direction. Apparent is the low variation in flux for both data sets shown of less then $1 \%$, visible by comparing the values of the normalized intensity in Fig. 5.6a. Figure 5.6b shows the transmitted intensity after interaction with the sample for the identical data points. The effect of the change of the transmission factor inside the device is visible, as data point 5 is the last point measured before the step, whereas data point 7 in the first one after the step. This effect is equally visible for the vimentin and the 

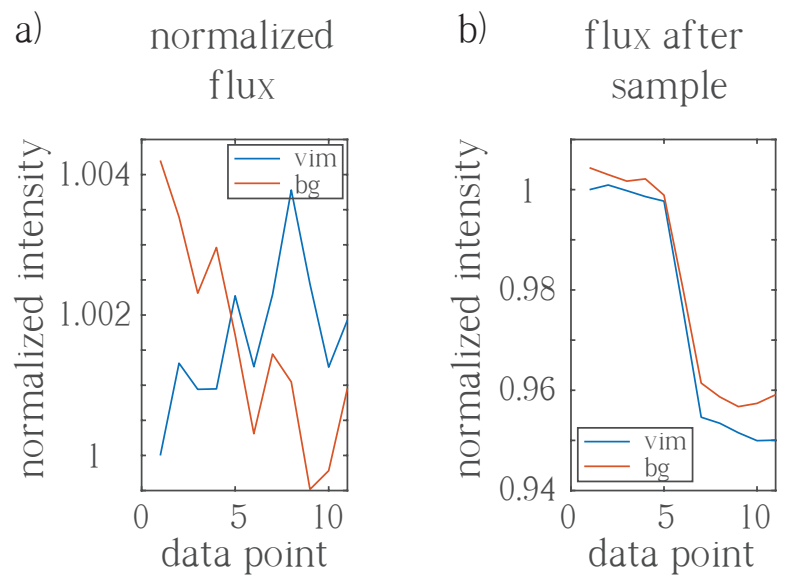

c) scaling factor

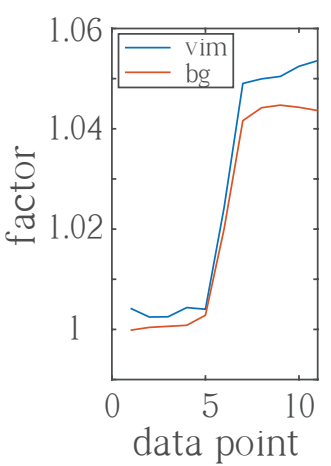

Figure 5.6.: Steps for calculating the correction factor for one set of cSAXS data. The signal for vimentin is shown in blue and the corresponding background measurement in red. a) The incident flux of the beamline is used to correct between different data sets for changes in the flux. b) Shows the transmitted photons through the device, indicating the absorbance of the device for different positions. The difference between the data points originates from the step. c) Calculated scaling factor for the first 11 data points of an exemplary data set.

background measurement. The decrease of transmitted intensity after the step is a direct result of an increase in aqueous solution in the beam path, as the $200 \mu \mathrm{m}$ of COC modelling the step in transmission direction are replaced with aqueous solution. Based on the flux prior to sample interaction and the transmitted flux, an individual scaling factor is calculated for all data points in each data set and applied prior to further analysis. Fig. 5.6c shows the corresponding scaling factor for the selected data points.

\subsubsection{Streak Finding Algorithm}

In addition to applying the correction factor calculated in Sec. 5.2.2, for each measuring point of the scanning-SAXS grid an individual mask is created. This mask is then used for all data analysis of data collected at this point, i.e., background and vimentin measurement. This is needed, as streak artifacts originating from the COC device superimpose signal on the vimentin scattering signal. Prior to azimuthal integration these streaks are filtered by applying an appropriate mask. Note, that one mask including all detected streaks of different data sets at the same measuring point is made and applied to all data collected at this measuring point. Each mask is created using a self-written MATLAB (The MathWorks, Inc., Natick, Massachusetts) script. Parts of the script are adapted from scripts of 


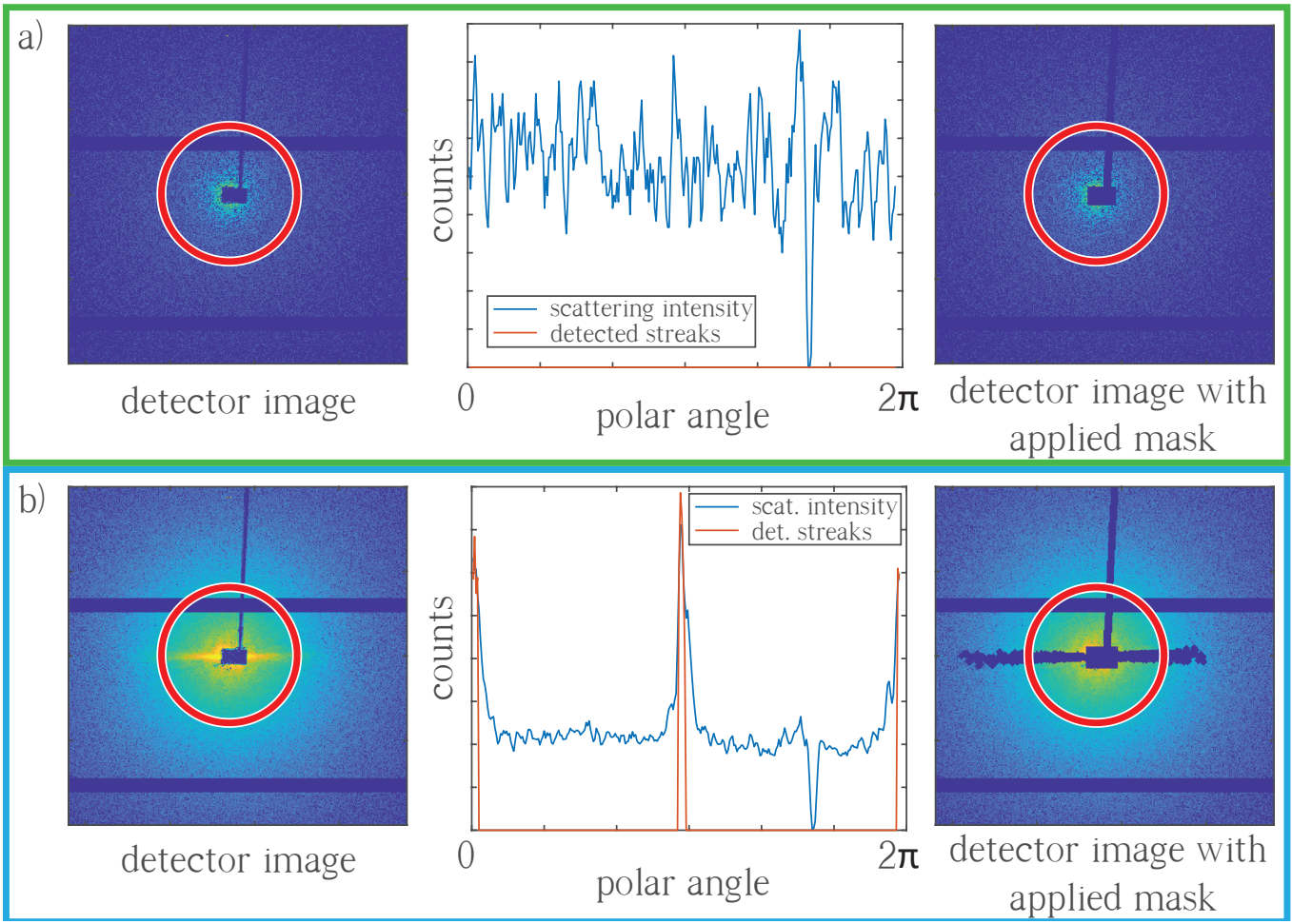

Figure 5.7.: Operating principle of the streak finder. Scattering images recorded by the detector indicate a streak in data set b), whereas data set a) contains no streak. The counted photons are colour coded with yellow representing the highest photon count (left). The radial intensity at the red circle is plotted (middle) and peaks are detected and masked for this scattering image (right).

previous members of the Institute for X-Ray Physics (Göttingen, Germany). The operating principle of this script is shown in Fig. 5.7a for a recorded scattering image with no streak, and in Fig. 5.7b for a recorded scattering image with a streak. For a recorded scattering image one radial scattering intensity $q$, indicated by the red circle (Fig. 5.7 left), is selected. The individual counts for each pixel are plotted against the polar angle (middle). In a classical analysis these pixels would be azimuthally integrated. Based on the distribution of the counts strong clustered increases are detected if occurring (Fig. 5.7b middle, red line), corresponding to the visible streak in Fig. 5.7b left. The corresponding pixel in the detector image is marked with its neighboring pixels and added to the individual mask. When no streak is detected, no additional pixel is masked, as a threshold of the mean with three standard deviations added is defined for outlier values. The right image in Fig. $5.7 \mathrm{a}+\mathrm{b}$ shows the created mask, after all radial scattering intensities are processed. The visible streak in Fig. 5.7b is now masked. 
Each scattering image of the collected vimentin data is subsequently masked with the masked related to the scanning position, azimuthally integrated, the correction factor is applied and background correction is performed with the corresponding scattering image of the background measurement, processed identically. The results for one data set are shown in the following.

\subsubsection{Time-Resolved Vimentin Assembly}

Time resolved scanning-SAXS measurements are performed at cSAXS to further investigate the kinetics of vimentin. By employing a step geometry inside the COC devices sample-surface interactions are prevented as discussed in Sec. 5.2.1.2. The acquired scattering profiles for selected points are shown in Fig. 5.8. The scattering intensities represent data collected from adjacent scanning points in the center of the device in the flow direction (see Fig. 5.2a, red line).

Assembly times points are given by estimating the scanning position inside the device based on the starting point of the measuring grid (see Fig. 4.6) and the data point number of the grid with the step size. This position is then correlated with the corresponding position from the simulation. For the simulation the $\mathrm{KCl}$ concentration, the flow velocity field and the mesh element sizes are known. The time needed for convective transport of vimentin to the measured position from the first point above the $\mathrm{KCl}$ threshold can be calculated, as described in Sec. 5.2.1.2. Note, that proper alignment of the device halves, shown in Fig. 4.3e+f, is critical to ensure the geometry of the device is described by the geometry used in the FEM simulations. To facilitate the alignment positive and negative cone structures, located on the periphery of the device, are used as aides when manually combining the halves with hot embossing. Thus, proper alignment of the devices halves ensures the time resolution of the simulation and experiment are in good agreement (see Sec. 4.2.1). All scattering patterns are individually masked, radially integrated and the different transmission factors for each data point are addressed as described in Sec. 5.2.3. Subsequently, the scattering profiles are background corrected with a corresponding background scattering profile recorded at the identical position. Due to variations of the streak artifacts for between sample and background measurement, $q$ values smaller than $0.06 \mathrm{~nm}^{-1}$ cannot be background subtracted despite the individual masking, as the background subtracted signal is not consistent.

The assembly of vimentin tetramers to ULFs is qualitatively visible by the in- 


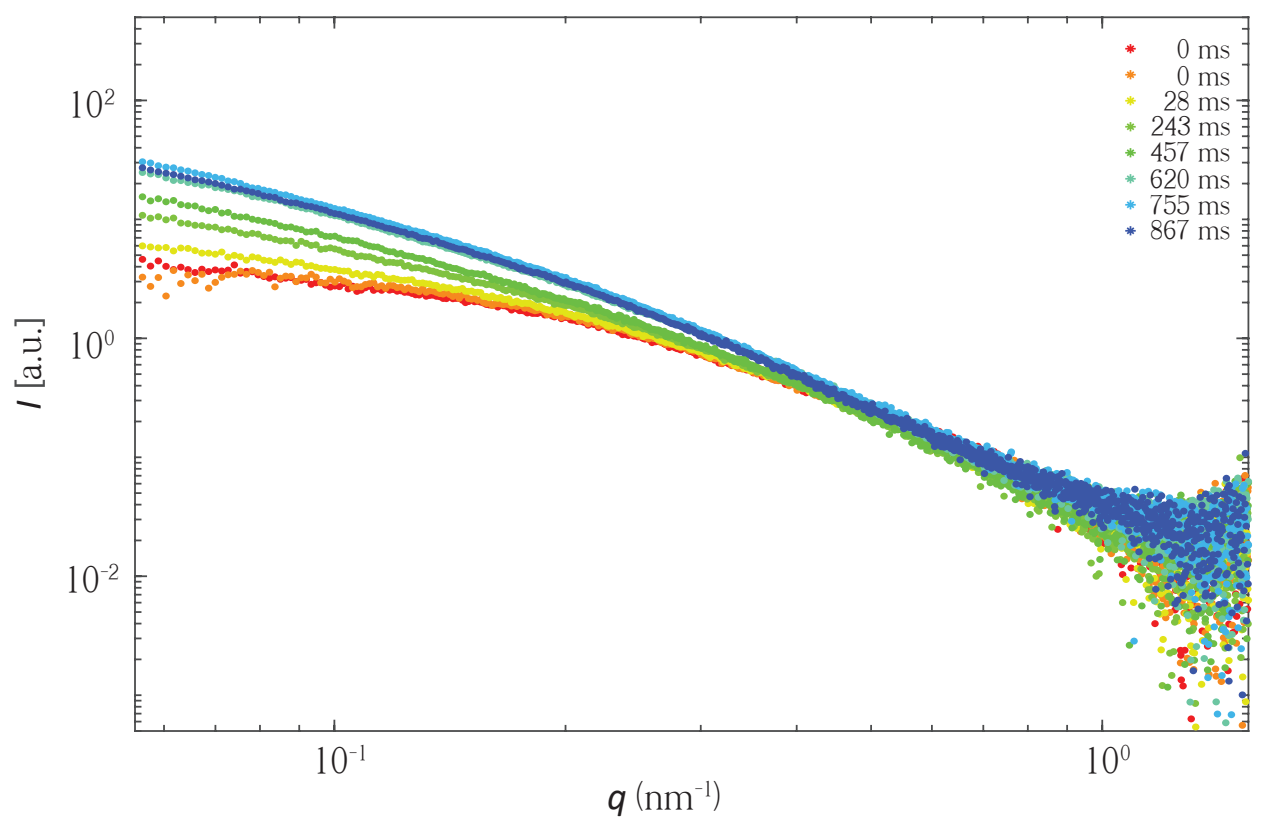

Figure 5.8.: Scattering profiles for vimentin measured inside a microfluidic device performed at cSAXS. Different colours correspond to different time points of the assembly. 0 ms indicate tetrameric vimentin.

crease of the forward scattering intensity $I_{0}$, as the molecular mass increased during assembly to ULFs. This has been demonstrated, e.g., by Brennich et al. [60].

The scattering signal of tetrameric vimentin is visible in the red and orange scattering curves. The signal for both data points is expected to be similar, as both are situated in the device before the threshold for assembly is reached. This threshold is set to $10 \mathrm{mM} \mathrm{KCl}$, as found by Brennich et al. [24]. Therefore, the assembly has not started and both scattering curves show the identical vimentin state. Starting with the yellow curve, representing $28 \mathrm{~ms}$ of assembly time, the increase of the projected $I_{0}$ is visible. The projected $I_{0}$ continues to increase monotonous for the remaining data points. This indicates an increase in the molecular weight. The scattering curve of the last shown point, displayed in blue shows no further increase of the projected $I_{0}$, indicating no further increase in molecular weight is occurring between the last 2 data points.

Although this increase in size during assembly is in agreement with literature $[24,103,104]$, the time scales for this to occur are not. ULFs are formed in the first $100 \mathrm{~ms}$ of vimentin assembly $[103,104]$. Thus, from the 4 th shown data set (see Fig. 5.8, light green, $243 \mathrm{~ms}$ ), no further change in the scattering curves should 
be observable. Since a difference is observed between the 4th and the following data points, the signal recorded is influenced by the device, despite the applied correction factors and masking. Therefore, no further analysis is performed on the recorded data. A possible explanation is discussed in Sec. 6.1.

The time resolution in the presented device could be improved by increasing the flow rates, decreasing the measurement step size or by changing the device geometry, e.g., decreasing the channel height. However, these options are limited: If the flow rates increase, the consumption of a finite sample volume increases. The measurement step size is designed in such a way as to minimize the Gaussian beam overlap from one scan position to another, which is limited by the beam size. With a constant flow rate, decreasing the channel height increases the flow velocities, and thus time resolution, but at a cost of decreasing the signal to noise ratio as fewer proteins are irradiated. 


\subsection{Small Molecules Inhibiting Vimentin Assembly}

The previous section of this work is dedicated to the assembly of vimentin to ULFs and how this kinetic can be accessed by simulations and in experiments with scanning-SAXS. However, due to the their key role in cell architecture, vimentin IFs need to reorganize in response to oxidants and electrophiles [33]. In this part the possibility of inhibiting vimentin assembly by using different substances is investigated. Different methods are employed to resolve the inhibiting effects on both filament radius and longitudinal assembly. SAXS is employed to access the impact on the filament radius. The longitudinal assembly and assembly inhibition is accessed over time via epi-fluorescent images. In a first step a tool is developed to access the length of vimentin filaments from microscope images in an automated fashion.

\subsubsection{Analysing Assembly Kinetics}

To analyse the impact of different chemicals on the assembly dynamics of vimentin, the length distribution from vimentin filaments is investigated. Vimentin samples are prepared as described in Sec. 4.4. A total of 1202 images are analysed for the different time points in the different data sets for the two chemical substances PBA and ACC. In the case of vimentin ACC interaction 125286 filaments and 8809 aggregates were detected and analysed, distributed among 590 images for the different ACC concentrations in three data sets (see Sec. 5.3.2 and appendix C). For the effect of PBA on vimentin a total of 147849 filaments and 8782 aggregates were detected and analysed among 612 images for the different conditions, contained in the three data sets (see Sec. 5.3.3 and Appendix B). The analysis is performed with a self-written MATLAB script. The script contains inbuild MATLAB functions for various tasks, e.g., REGIONPROPs for object detection, EDGE for edge detection, BWDISTGEODESIC for the quasi-euclidean distance. The algorithm allows to analyse large numbers of images, as no specific user input is required for operation, as described in the following. 


\subsubsection{Structure-Detecting Algorithm}

Raw images from the microscope are read in and the intensity distribution is normalized to grey values between 0 and 1 , with 1 being white and 0 black. Contrast is enhanced by removing outliers prior to normalization and subsequently adding them with the maximum value afterwards. An exemplary section of an normalized image is shown in Fig. 5.9a. The normalized image is noise reduced by a $6 \times 6$ pixels Wiener filter. A Gaussian filter with $\sigma=1.5$ is employed for edge enhancement. An adaptive Canny edge finder is used to detect the edges of the structures displayed in Fig. 5.9b. After the edge detection the image is binarised. Adaptive morphological image operations, e.g., dilution followed by erosion with the identical structuring element, are used to connect the detected edges prior to filling the detected structures. Objects at the image edge are removed, as no full information on these is known. The result is shown in Fig. 5.9c. Each of the objects in the binarised image is handled individually for the following process. First, the overall size in pixel of the object is determined. Objects smaller than 50 pixels are

a)

$$
\begin{gathered}
\text { normalized } \\
\text { image }
\end{gathered}
$$

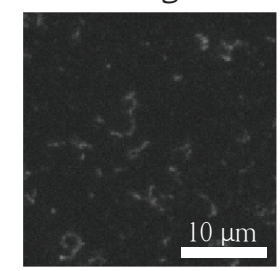

d)

detected

filaments

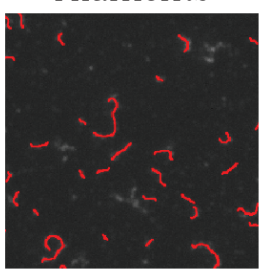

b)

$$
\begin{gathered}
\text { detected } \\
\text { edges }
\end{gathered}
$$

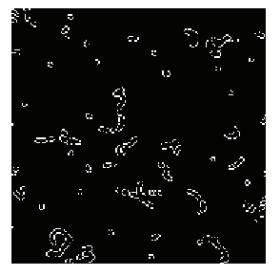

e)

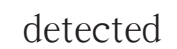

aggregates

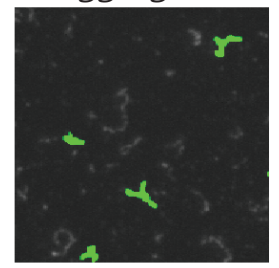

c) filtered, binarized and filled

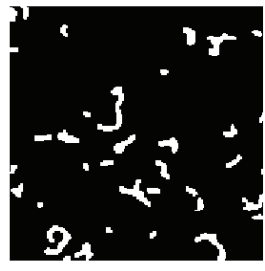

f) detected short pieces

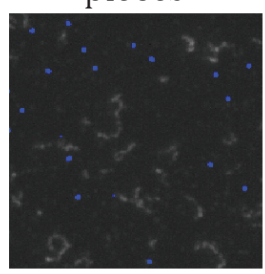

g) detected

structures

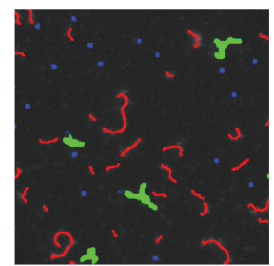

Figure 5.9.: Processing steps of a raw microscope image during structure detection. a) The image is contrast enhanced, noise reduced and edge enhanced prior to b) edge detection. Adaptive morphological image operations connect the detected edges and enable the structures to be filled d). Each object is analysed and assigned to different categories. Filament length are calculated d), whereas for aggregates the size in pixel in measured. f) Short pieces represent objects too small to be consistently analysed. An overview of the detected structures is shown in $\mathrm{g}$ ). 
excepted from subsequent analysis, as they are often produced by artifacts from the edge-finding algorithm. To put this size into perspective, analysed aggregates frequently consist of more than 1000 pixels (see Fig. 5.13 and Fig. 5.19). One pixel corresponds to $66 \times 66 \mathrm{~nm}^{2}$ in real space. Second, the binary shape is reduced to a one pixel connected structure which is still representative of the shape's original form. This pixel line is called a 'skeleton' as the essential information about the object is conserved, e.g., Euler number and topology are conserved. All endpoints of this skeleton are traced and the distance along the pixel line to all other endpoints is calculated. This is done for each endpoint resulting in finding the two endpoints representing the longest connected part of the original object with one start and one endpoint. This pixel line is the found filament structure and shown red in Fig. 5.9d for each detected filament.

The distance of this connection is calculated with a quasi-euclidean distance norm following the connecting one pixel line of the skeleton. To distinguish if the found length truly represents a filamentous object, the found connection is enlarged with a disc structure element larger than the expected width of a filament. The original object is masked with this found filament length and if pixels remain after masking the object is not clearly represented by the found connection and the assumption of a filamentous shape. The object is then defined as an aggregate. Examples for detected filaments and detected aggregates are shown in Fig. $5.9 \mathrm{~d}+\mathrm{e}$, respectively.

An overview of all detected structures is shown in Fig. 5.9g. The calculated length as well as the sizes of the aggregates are saved for further analysis, addressed in Sec. 5.3.2.1 and Sec. 5.3.3.1.

\subsubsection{Benchmarking}

Prior to further analysis of the length and the pixel sizes measured the algorithm is tested by using a test data set containing different geometrical objects. Figure 5.10a-c shows the three different shapes, each represented in different scaled variations. The forms are half circles, 3/4 circles and sine curves. Sizes are chosen to represent pixel length occurring in the measured microscopy data. The arclength of these objects is known, as they can be analytically expressed. The arclength of a differentiable function $f$ on the interval $[a, b]$ is defined as $\int_{a}^{b} \sqrt{1+\left(f^{\prime}(x)\right)^{2}} d x$ [105]. For half circles and $3 / 4$ circles the arclength is trivial and for the sine curves the integral is numerically solved with MATLAB. The analytical length is compared to the result of the algorithm, shown in Fig. 5.10d-f. Beside the quasi- 
a)

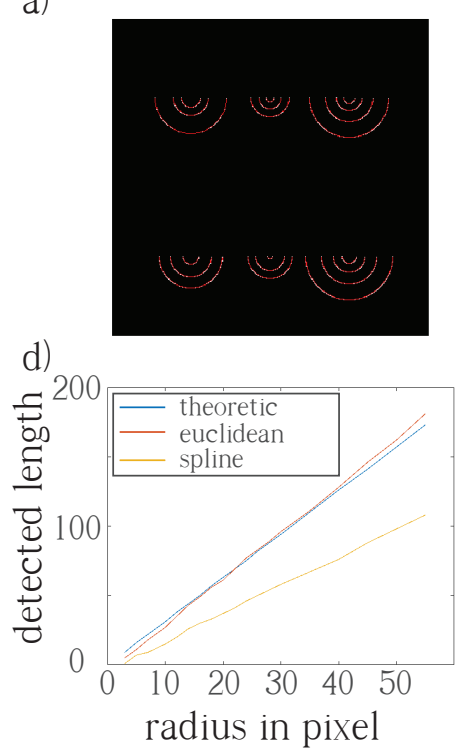

b)
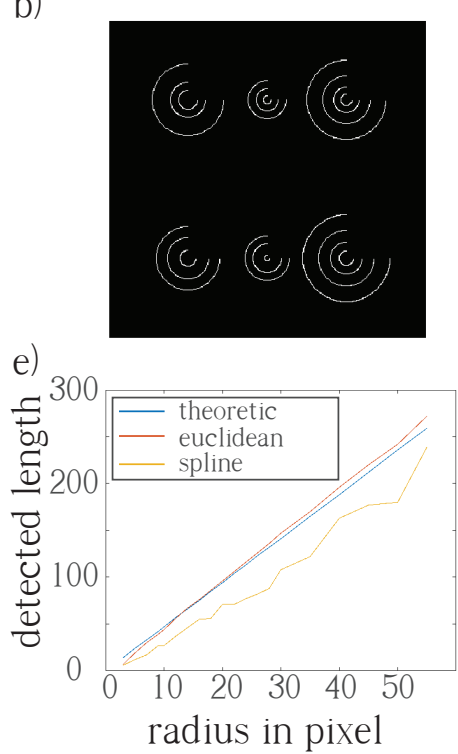

c)

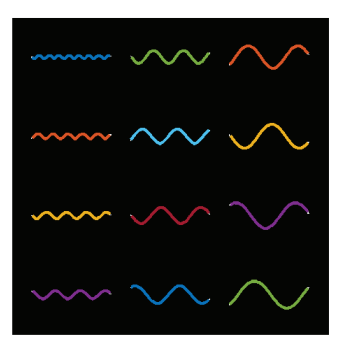

f)

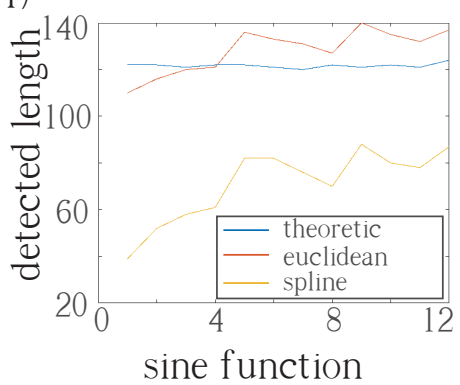

Figure 5.10.: Different geometric structures including half circles a), 3/4 circles b) and sine curves c) in different sizes. For each geometric object the length is analytically calculated and compared to the length detected by the algorithm using a quasi-euclidean norm and a spline for the distance.

euclidean distance a spline is used to find the objects and the spline length is given in addition to the quasi-euclidean length. Figure 5.10a further shows the detected filament length for the half circles in red, whereas Fig. 5.10c displays the found splines for the sine curves. The sine curves are numbered in a column wise manner. The upper left sine curve is referred to as 1 , whereas the lower right sine curve is referred to as 12 . The number of the sine function is given on the sine function axis with the corresponding length on the detected length axis.

For all three tested geometries with different sizes the quasi-euclidean distance, plotted in red, and the distance calculated with splines, shown in yellow are given. The difference between the calculated lengths and the theoretical length, shown in blue, is consistently smaller for the quasi-euclidean distance. Thus, the quasieuclidean distance is used in the algorithm for the length calculation of the detected objects, as the measured values are closer to the known length of the test objects. In the following the results for 2 exemplary data sets, one each for ACC and PBA, are analysed individually. A comparison of the presented data sets and to literature is given in Sec. 5.3.4. 


\subsubsection{Vimentin Assembly in the Presence of N-Acetyl-L-Cysteine}

In the following, the results of vimentin assembled with varying concentrations of ACC, are presented. All vimentin samples are prepared in $5 \mathrm{mM}$ TRIS buffer with $100 \mathrm{mM} \mathrm{NaCl}$ inducing the assembly and are incubated at $37^{\circ} \mathrm{C}$ for $1 \mathrm{~h}, 2$ $\mathrm{h}, 4 \mathrm{~h}, 8 \mathrm{~h}$, and $24 \mathrm{~h}$ prior to imaging. The sample preparation is described in detail in Sec. 4.1.3. After incubation, the microscopy slides are prepared with the solutions containing different ACC concentrations and are subsequently imaged. Details on the epi-fluorescent data acquisition are given in Sec. 4.4. Exemplary microscopy images are shown in Fig. 5.11a-d on the left side. All images shown are taken after $4 \mathrm{~h}$ of assembly time at $37^{\circ} \mathrm{C}$ with varying concentrations of ACC. Figure 5.11a contains no ACC, Fig. 5.11b $5 \mathrm{mM}$ ACC, Fig. 5.11c $20 \mathrm{mM}$ ACC and Fig. $5.11 \mathrm{~d} 50 \mathrm{mM}$ ACC. The results of the structure detection algorithm are overlayed on the corresponding microscopy image. Aggregates are shown in green, whereas the detected filaments are shown in red. For $0 \mathrm{mM} \mathrm{ACC}$, see Fig. 5.11a, the vimentin has assembled to filaments. With increasing length, the filaments show more overlap, as a single filament contain loops. These cases are detected as aggregates, as described in Sec. 5.3.1.1. For $5 \mathrm{mM} \mathrm{ACC}$, see Fig. 5.11b, both the length of the detected filaments as well as the amount and size of the detected aggregates decrease. The vimentin incubated with $20 \mathrm{mM} \mathrm{ACC}$ has not formed filaments, see Fig. 5.11c, neither aggregates are visible for this ACC concentration. In Fig. 5.11d, showing the vimentin incubated with the highest ACC concentration, no filaments are visible, but aggregates have formed. These aggregates do not consist of filamentous structures, as the aggregates for the $0 \mathrm{mM}$ and $5 \mathrm{mM}$ condition. To confirm the described visual inspection, the recorded data is analysed with the structure detecting algorithm. The visualisations of the results are discussed in detail in the following for one data set. The results for the remaining data sets for the identical experiment are given in the appendix $C$.

\subsubsection{Length Distributions}

Microscopy images are processed with the structure detecting algorithm and the detected lengths are stored. A total of 175 recorded images for the different concentrations and time points are analysed for this data set. Figure 5.12 contains the length distributions in $\mu \mathrm{m}$ of the detected filaments as a box plot. Filament lengths from images recorded for the same incubation time and ACC concentration are grouped, resulting in one box per condition. A total of 34452 filaments are 
a)
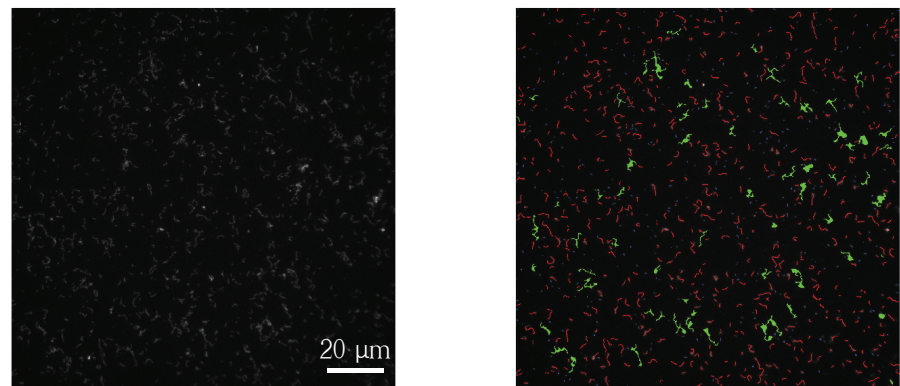

0 mM ACC, 100 mM NaCl, 4 hours assembly

b)
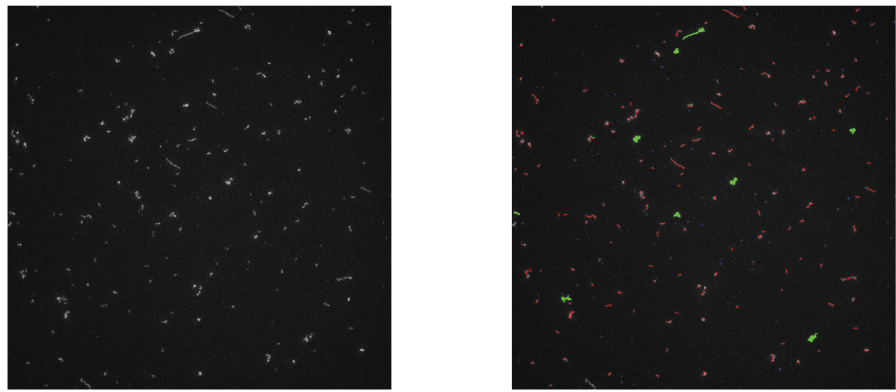

5 mM ACC, 100 mM NaCl, 4 hours assembly

c)
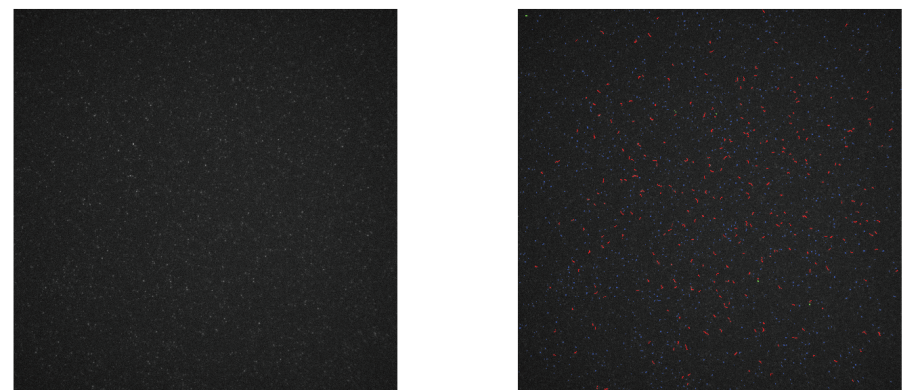

20 mM ACC, 100 mM NaCl, 4 hours assembly

d)
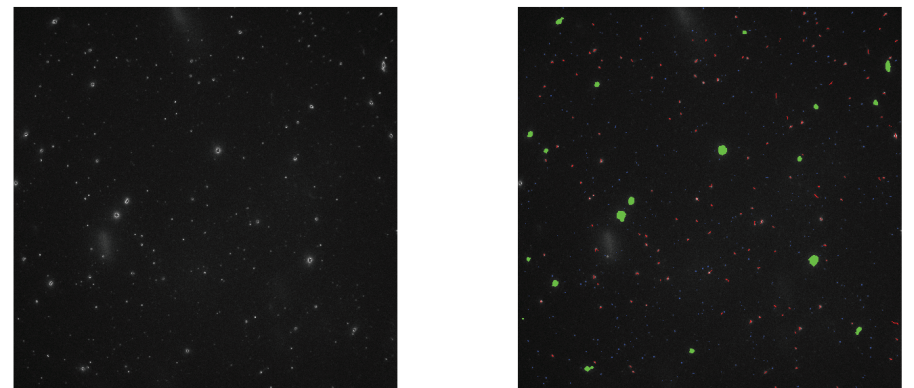

50 mM ACC, 100 mM NaCl, 4 hours assembly

Figure 5.11.: Epi-fluorescence images of vimentin after 4 hours at $37{ }^{\circ} \mathrm{C}$ in $5 \mathrm{mM}$ TRIS with $100 \mathrm{mM} \mathrm{NaCl}$ and varying concentration of ACC (left side). Results of the structures detected by the detection algorithm are shown on the right. Filaments are shown in red, aggregates in green. Concentration of ACC increases from top to bottom, with a) containing $0 \mathrm{mM} \mathrm{ACC}, \mathrm{b}$ ) $5 \mathrm{mM} \mathrm{ACC}, \mathrm{c}) 20 \mathrm{mM}$ ACC and d) $50 \mathrm{mM} \mathrm{ACC}$. 


\section{\begin{tabular}{l|l} 
Chapter 5 & RESULTS
\end{tabular}}

contained in Fig. 5.12. The exact amount of filaments detected for each condition is shown in Tab. 5.2, e.g., the first blue data entry of the box plot in Fig. 5.12, the filament length distribution for vimentin incubated with $0 \mathrm{mM} \mathrm{ACC}$ for 1 hour, contains 3964 filaments (see Tab. 5.2 intersection of 1 hour assembly time and 0 $\mathrm{mM}$ ACC concentration). The central mark of each box in Fig. 5.12 indicates the median for the filament length distribution of this condition. The top and bottom edges of each box indicate the 75th and 25th percentiles, respectively. Lengths longer or shorter than the 75th and 25th percentiles, not considering outliers, are indicated by the whiskers. Measured filament lengths are defined outliers if their length is greater than $p_{75}+\mathrm{w} \times\left(p_{75}-p_{25}\right)$ or less than $p_{25}-\mathrm{w} \times\left(p_{75}-p_{25}\right)$, with $p_{25}$ and $p_{75}$ representing the 25 th and 75 th percentiles and $w$ being the maximum whisker length.

Figure 5.12 shows an increase in filament lengths for $0 \mathrm{mM} \mathrm{ACC}$ (blue) over time. For $5 \mathrm{mM}$ ACC (green) a smaller increase in filament length is visible over time. In addition, the median length after $24 \mathrm{~h}$ is shorter than the one after $8 \mathrm{~h}$ incubation time (1.2 $\mu \mathrm{m}$ compared to $1.8 \mu \mathrm{m})$. A possible explanation is addressed with the detected aggregate sizes in the following section. For both the $20 \mathrm{mM}$ ACC and the $50 \mathrm{mM}$ ACC concentrations no change in the length distribution over time is visible. For each time point the average filament length is smaller for vimentin incubated with $5 \mathrm{mM}$ ACC than vimentin incubated with no ACC. 


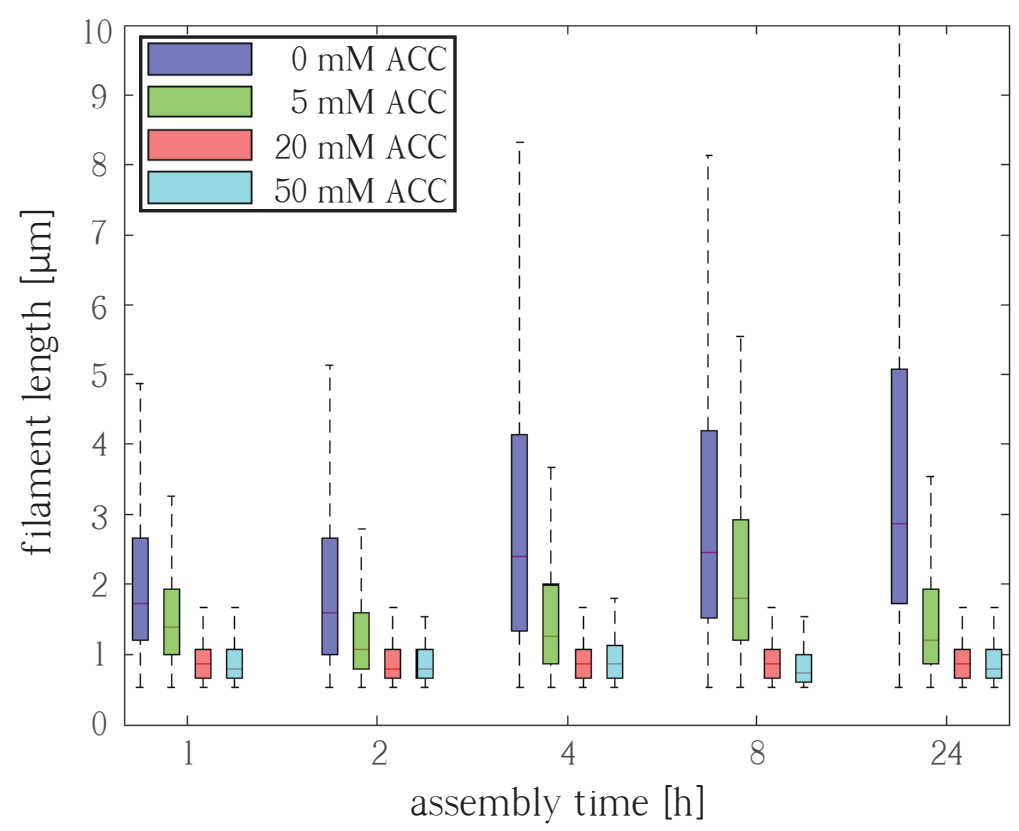

Figure 5.12.: Length distributions of vimentin tetramers incubated with different ACC concentrations over $1 \mathrm{~h}, 2 \mathrm{~h}, 4 \mathrm{~h}, 8 \mathrm{~h}$, and $24 \mathrm{~h}$. For each time point the related length distribution in $\mu \mathrm{m}$ is box plotted, the central mark indicating the median, and bottom and top edges of the box indicating the 25th and 75th percentiles, respectively. $0 \mathrm{mM} \mathrm{ACC}$ is shown in blue, $5 \mathrm{mM}$ ACC in green, $20 \mathrm{mM} \mathrm{ACC}$ in red and $50 \mathrm{mM} \mathrm{ACC}$ in turquoise. The number of filaments for each data point is shown in Tab. 5.2.

Table 5.2.: Number of filaments detected for the different ACC concentration for each time point. The number includes filaments from multiple images taken for the same condition, e.g., 1948 filaments for $5 \mathrm{mM} \mathrm{ACC}$ after $2 \mathrm{~h}$ of assembly. Length distribution in $\mu \mathrm{m}$ for each condition are shown in Fig. 5.12.

\begin{tabular}{|l|l|r|r|r|r|}
\hline & \multicolumn{5}{|c|}{ ACC concentration } \\
\hline & & $0 \mathrm{mM}$ & $5 \mathrm{mM}$ & $20 \mathrm{mM}$ & $50 \mathrm{mM}$ \\
\cline { 2 - 6 } assembly time & 1 hour & 3964 & 4158 & 114 & 505 \\
\cline { 2 - 6 } & 2 hours & 1551 & 1948 & 900 & 220 \\
\cline { 2 - 6 } & 4 hours & 2196 & 2696 & 4030 & 1715 \\
\cline { 2 - 6 } & 8 hours & 1121 & 1763 & 1830 & 615 \\
\cline { 2 - 6 } & 24 hours & 906 & 920 & 2289 & 1011 \\
\hline
\end{tabular}




\section{\begin{tabular}{l|l} 
Chapter 5 & RESULTS
\end{tabular}}

\subsubsection{Aggregate Distributions}

In addition to the length distributions the aggregate sizes in pixel are measured for each image. One pixel has the size of $66 \times 66 \mathrm{~nm}$. For all twenty conditions $(4$ concentrations $\times 5$ time points) a total of 2941 aggregates are detected, grouped by condition and box plotted in Fig. 5.13. Additional data sets are shown in the appendix C. The number of aggregates included in each box of Fig. 5.13 is shown in Tab. 5.3. The median, indicated by the horizontal bar in each box, increases over time for vimentin incubated with $0 \mathrm{mM}$ ACC. Supporting this increase in pixel size is the increasing trend of the edges of the 75th and 25th percentiles. The same trend in increasing aggregate size and median is observable for the $5 \mathrm{mM}$ ACC condition, shown in green.

The overall size of these aggregates is smaller than the aggregate size measured for vimentin incubated with $0 \mathrm{mM}$ ACC. The difference of the 75th percentile to the median for $24 \mathrm{~h}$ hours of incubation time is approximately doubled compared to the same difference for the other time points of the same ACC concentration, indicating a broad variation of structures much larger than the average size. A closer look on the images recorded for $0 \mathrm{mM}$ ACC reveals multiple $\mu \mathrm{m}$ long filaments being detected as aggregates, as they self-overlap in various parts. This corresponds to the observation for the filament length of the same $5 \mathrm{mM} \mathrm{ACC}$ after $24 \mathrm{~h}$ condition in Fig. 5.12, where the median decreased compared to the $8 \mathrm{~h}$ measurement from $1.8 \mu \mathrm{m}$ to $1.2 \mu \mathrm{m}$. Further supported is this finding by the additional data sets shown in the appendix $C$. In these data sets, the aggregate size distribution does not change between $8 \mathrm{~h}$ and $24 \mathrm{~h}$ hours.

For vimentin incubated with $20 \mathrm{mM}$ ACC few and small aggregates are detected for all conditions, indicating no aggregate formation compared to the other conditions, e.g., 13 aggregates with an average size of 69 pixels for $4 \mathrm{~h}$ of incubation time (see Tab. 5.3 and Fig. 5.13). The small size is further indicated by the low median and the dense concentration of the measured aggregate sizes around the median, visible by the 25th and 75th percentiles being close to the median. Aggregate sizes for $50 \mathrm{mM}$ ACC are shown in turquoise in Fig. 5.13. The median is approximately constant for 2 h, 4 h, 8 h, and 24h (544 pixels, 655 pixels, 582 pixels, 682 pixels, respectively), whereas the median aggregate size for $50 \mathrm{mM}$ ACC incubation after $1 \mathrm{~h}$ is with 909 pixels significantly larger. This increase is unique to this data set and considered an artifact, as both data sets in the appendix $C$ do not show this increase. 


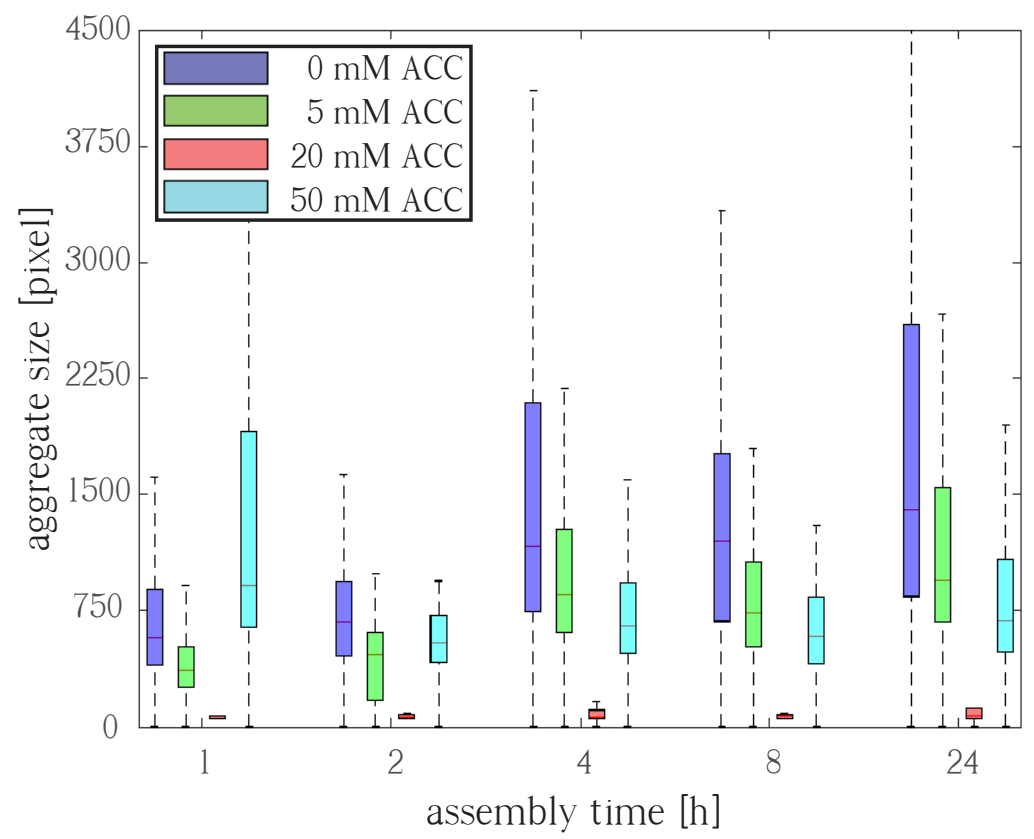

Figure 5.13.: Aggregate distributions of vimentin tetramers incubated with different ACC concentrations over $1 \mathrm{~h}, 2 \mathrm{~h}, 4 \mathrm{~h}, 8 \mathrm{~h}$, and $24 \mathrm{~h}$. For each time point the related aggregate distribution in pixels is box plotted, the central mark indicating the median, and bottom and top edges of the box indicating the 25th and 75th percentiles, respectively. $0 \mathrm{mM} \mathrm{ACC}$ is shown in blue, $5 \mathrm{mM} \mathrm{ACC}$ in green, $20 \mathrm{mM} \mathrm{ACC}$ in red and $50 \mathrm{mM} \mathrm{ACC}$ in turquoise. The number of aggregates for each data point is shown in Tab. 5.3.

Table 5.3.: Number of aggregates detected for the different ACC concentrations for each time point, e.g., 43 aggregates for $20 \mathrm{mM} \mathrm{ACC}$ after $4 \mathrm{~h}$ of assembly. Aggregate size distribution in pixels for each condition are shown in Fig. 5.13.

\begin{tabular}{|l|l|r|r|r|r|}
\hline & \multicolumn{5}{|c|}{ ACC concentration } \\
\hline & & $0 \mathrm{mM}$ & $5 \mathrm{mM}$ & $20 \mathrm{mM}$ & $50 \mathrm{mM}$ \\
\cline { 2 - 7 } assembly time & 1 hour & 410 & 151 & 6 & 71 \\
\cline { 2 - 7 } & 2 hours & 133 & 47 & 13 & 22 \\
\cline { 2 - 7 } & 4 hours & 472 & 354 & 43 & 144 \\
\cline { 2 - 7 } & 8 hours & 251 & 174 & 13 & 51 \\
\cline { 2 - 6 } & 24 hours & 278 & 202 & 25 & 81 \\
\hline
\end{tabular}




\section{\begin{tabular}{l|l} 
Chapter 5 & RESULTS
\end{tabular}}

Note, that both the number and size of aggregates increases for vimentin incubation with $50 \mathrm{mM}$ ACC compared to $20 \mathrm{mM}$ ACC for all incubation times investigated, whereas the measured filament lengths remain below $1 \mu \mathrm{m}$ for both cases for all time points investigated. Furthermore, the aggregate size decreases in a monotonic manner for all time points from $0 \mathrm{mM}$ ACC to $5 \mathrm{mM}$ ACC until $20 \mathrm{mM}$ ACC, where all aggregates disappeared.

\subsubsection{Molecular Mass and Radius of Gyration}

Inhouse SAXS experiments are performed on vimentin incubated with ACC at 0 $\mathrm{mM}, 5 \mathrm{mM}, 20 \mathrm{mM}$ and $50 \mathrm{mM}$. Sample preparation and measuring procedure are described in Sec. 4.3.3.1, whereas data treatment is described in Sec. 4.3.4. In Fig. 5.14 the scattering profiles for vimentin assembled with ACC are shown. Each ACC concentration was measured 3 times and Fig. 5.14 contains all three data sets. The scattering profile for $50 \mathrm{mM}$ ACC shows a different form then the ones recorded for $0 \mathrm{mM}$ ACC, $5 \mathrm{mM}$ ACC and $20 \mathrm{mM} \mathrm{ACC}$, as around $q=0.05 \mathrm{~nm}^{-1}$ an upturn is visible. This upturn indicates aggregation in the sample [106], thus the

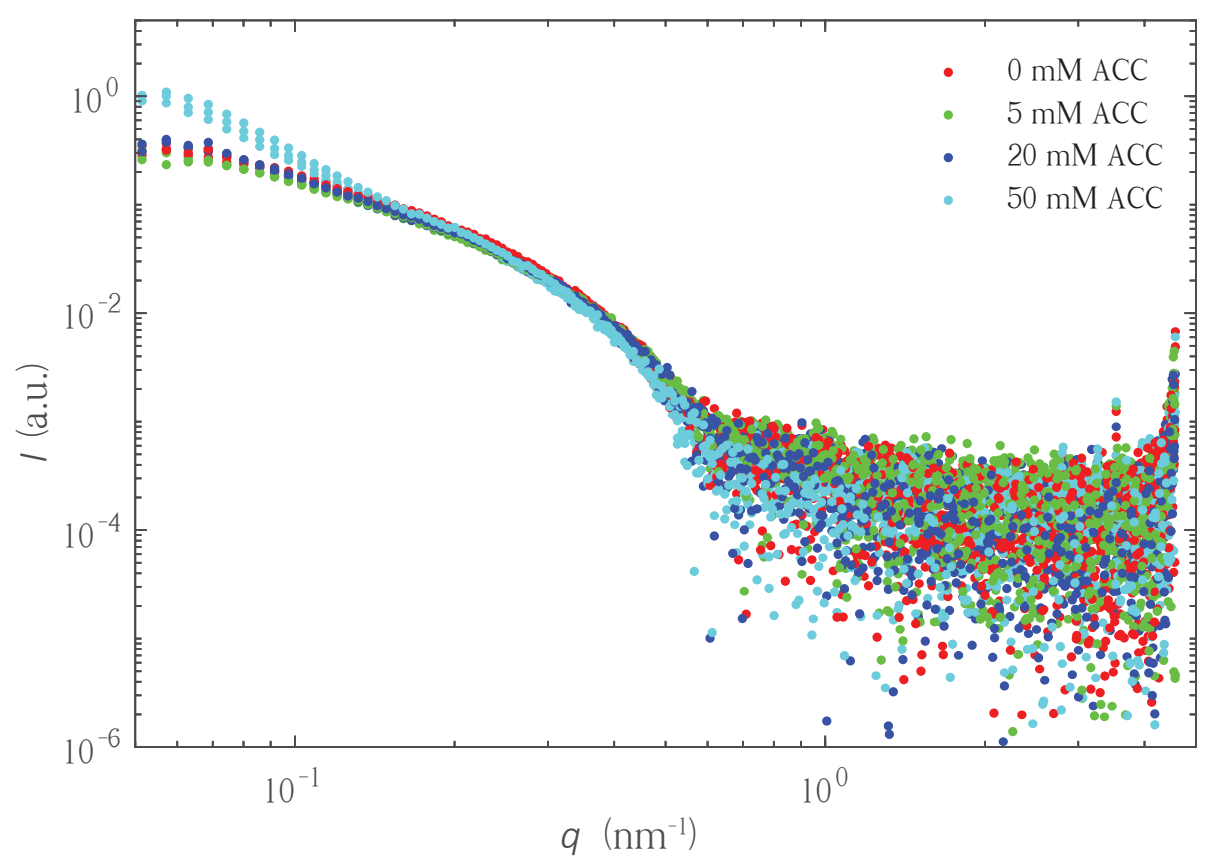

Figure 5.14.: Scattering profiles of vimentin assembled with various ACC concentrations. ACC concentrations range from $0 \mathrm{mM} \mathrm{ACC}$ over $5 \mathrm{mM}$ and $20 \mathrm{mM}$ to $50 \mathrm{mM}$. For each concentration 3 independent measurements are shown. 
applied model for Guinier's analysis with no interactions between neighbouring particles cannot be applied as the system is not diluted. Therefore, no $R_{G}$ or $I_{0}$ are given for $50 \mathrm{mM}$ ACC in the following. Guinier's analysis is performed in the Guinier region $q R_{G}<1.3$ as described in Sec. 3.2.3 using the PRIMUS (ATSAS, EMBL, Hamburg, Germany) software package. Exemplary Guinier fits are shown for three ion-induced vimentin self-assembly measurements containing $5 \mathrm{mM}$ ACC in Fig. 5.15a-c. The scattering data is shown in blue, whereas the Guinier fit is displayed in turquoise. For each fit the corresponding normalised fit residual is given in Fig. 5.15d-f. For the first data set shown in Fig. 5.15a $R_{G}=5.24 \mathrm{~nm}$, whereas the Guinier analysis for the data shown in Fig. $5.15 \mathrm{~b}+\mathrm{c}$ yields $R_{G}=5.26 \mathrm{~nm}$ and $R_{G}=5.31 \mathrm{~nm}$, respectively. The results for the all data sets containing ACC are shown in Fig. 5.16. Fig. 5.16a shows a slight increase in $R_{G}$ for $20 \mathrm{mM}$ ACC for each data set. The mean $\overline{R_{G}}$ for $0 \mathrm{mM} \mathrm{ACC}$ is $5.33 \mathrm{~nm}$, for $5 \mathrm{mM} \mathrm{ACC} \overline{R_{G}}=5.27 \mathrm{~nm}$, and for $20 \mathrm{mM} \overline{R_{G}}=6.08 \mathrm{~nm}$. An increased $R_{G}$ indicates larger structures in the sample for higher ACC concentrations. The interpolated $I_{0}$ is shown in Fig 5.16b indicates the molecular weight of the scatterer. The $I_{0}$
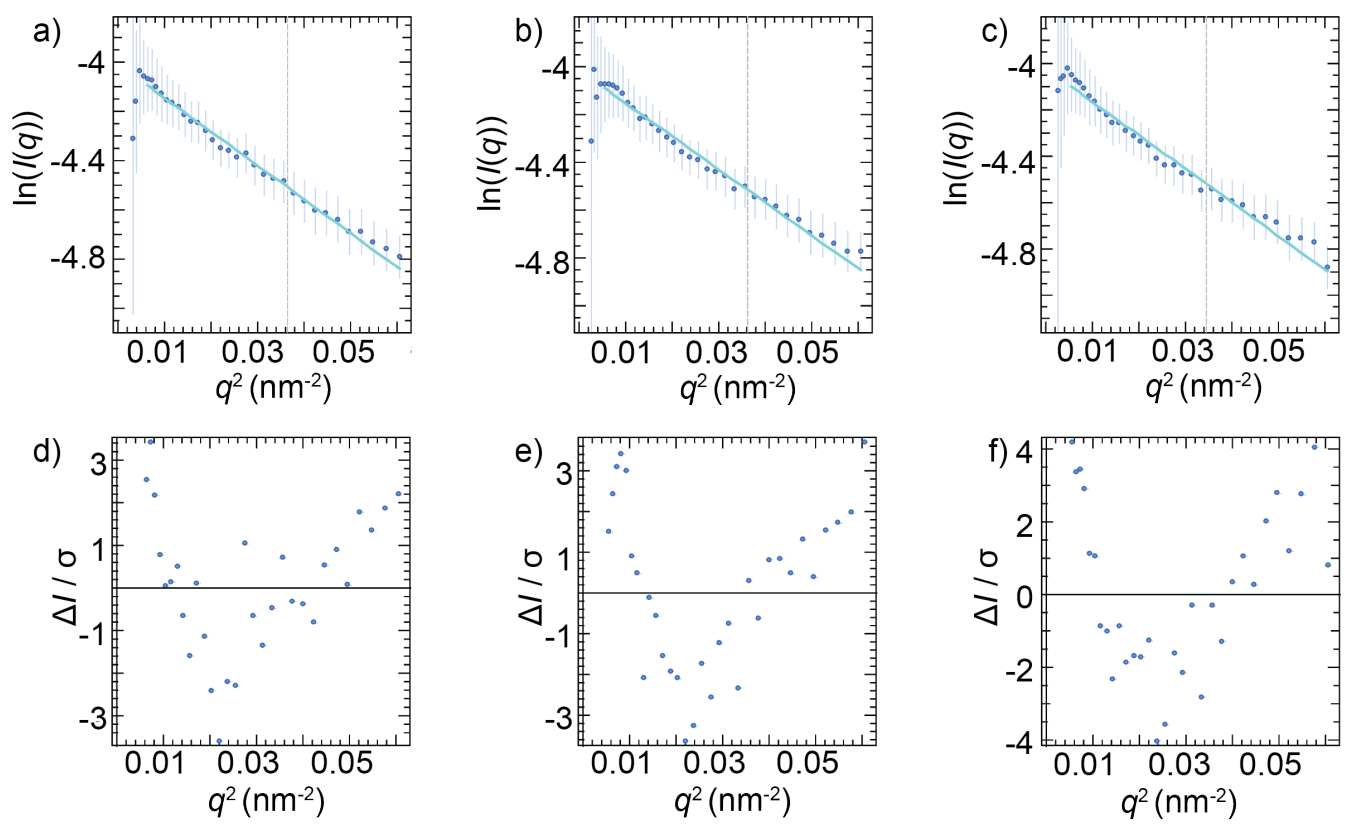

Figure 5.15.: Guinier fits for three ion-induced vimentin self-assembly measurements containing 5 mM ACC. a)-c) scattering data shown in blue with the corresponding Guinier fit in turquoise. d)-f normalised fit residual for the Guinier fits. The data analysis yields $R_{G}=5.24 \mathrm{~nm}$ for a) $R_{G}=5.26 \mathrm{~nm}$ for $\mathrm{b}$ ) and $R_{G}=5.31 \mathrm{~nm}$ for $\mathrm{c}$ ). 


\section{\begin{tabular}{l|l} 
Chapter 5 & RESULTS
\end{tabular}}
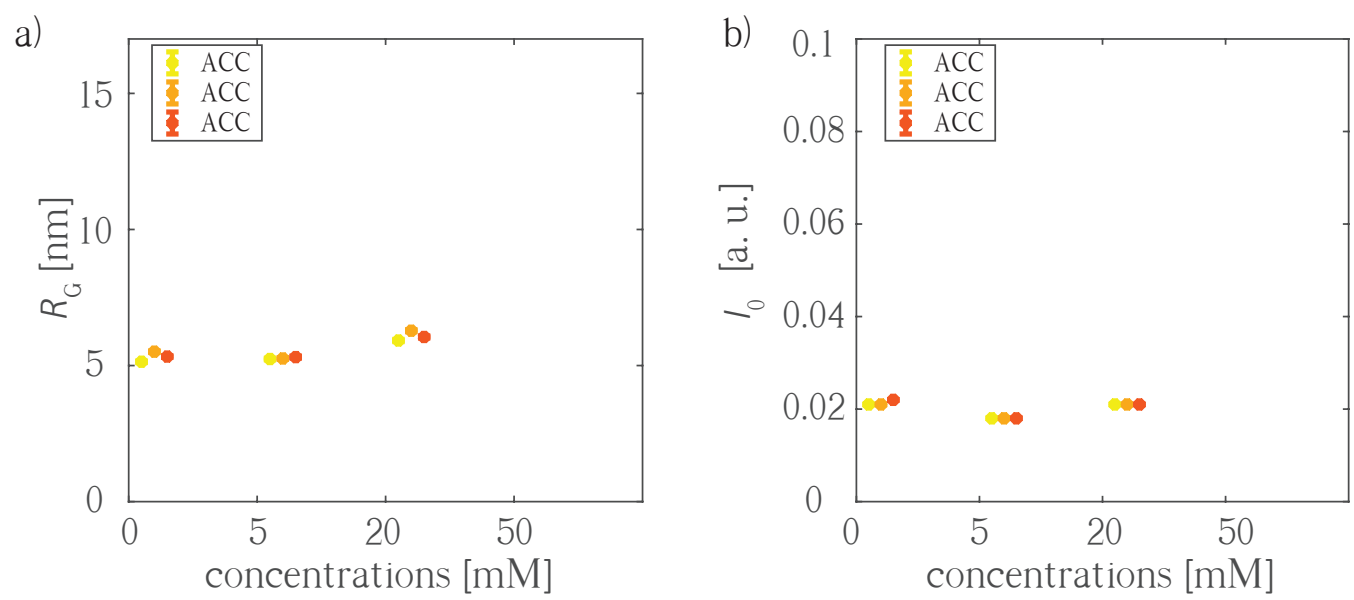

Figure 5.16.: Guinier analysis for the scattering profiles shown in Fig. 5.14. a) The radius of gyration is calculated and shown for each ACC concentration ranging from $0 \mathrm{mM}$ over $5 \mathrm{mM}$ to $20 \mathrm{mM}$. The different data sets are colour coded, with one colour showing the $R_{G}$ for the different conditions. An increase of $R_{G}$ for $20 \mathrm{mM} \mathrm{ACC}$ concentration is visible. b) Calculated forward scattering intensity $I_{0}$ for the colour coded 3 different data sets. No clear difference in $I_{0}$ between the shown concentrations is observable.

shown in Fig 5.16b is in arbitrary units, as the exact molecular weight cannot be calculated, but as all data is taken on the same set up, thus the same CF applies, the $I_{0}$ can be compared to each other. A slight increase in $I_{0}$ is observed for the 20 $\mathrm{mM}$ ACC concentrations, which is in agreement with the $R_{G}$ shown in Fig. 5.16a. 


\subsubsection{Vimentin Assembly in the Presence of Sodium Phenylbutyrate}

The second chemical compound investigated regarding its influence of vimentin assembly is PBA. All assembly experiments with PBA are performed in the identical manner as the vimentin assembly experiments with ACC described in Sec. 5.3.2, only substituting the ACC with PBA. Exemplary images from vimentin after $4 \mathrm{~h}$ of incubation at $37^{\circ} \mathrm{C}$ in $5 \mathrm{mM}$ TRIS, $100 \mathrm{mM} \mathrm{NaCl}$ and different concentrations of PBA, ranging from $0 \mathrm{mM}$ over $5 \mathrm{mM}$ and $20 \mathrm{mM}$ to $50 \mathrm{mM}$ are shown in Fig. 5.17. On the left side of the figure the recorded raw images are shown, whereas the right side contains the results of the structure detection algorithm. Detected filamentous structures are shown in red and aggregates in green. Figure 5.17a displays the vimentin incubated without PBA, whereas the concentration of PBA gradually increases over $5 \mathrm{mM}$ PBA in Fig. $5.17 \mathrm{~b}$ and $20 \mathrm{mM}$ PBA in Fig. 5.17c to $50 \mathrm{mM}$ PBA in Fig. 5.17d. The time point shown is selected to enable comparison to Fig. 5.11.

The length of vimentin filaments and the size of aggregates are similar for the $0 \mathrm{mM}$ PBA, $5 \mathrm{mM}$ PBA and $20 \mathrm{mM}$ PBA. Based solely on the microscope image no effect from the PBA on the vimentin assembly is visible. This changes for the highest concentration with $50 \mathrm{mM}$ PBA, shown in Fig. 5.17d. Both the filaments and the aggregates are no longer present. To quantify the length distributions and aggregate sizes for the different incubation times and PBA concentrations the structure detection algorithm is used and the results are presented in the following.

\subsubsection{Length Distributions}

Calculated filament length distributions in $\mu \mathrm{m}$ for the observed concentrations and time points are given in Fig. 5.18. A total of 33490 filaments are detected for the combined 179 images representing the different time points and concentrations in this data set. Results from images belonging to one condition are added. Detailed information on how many filaments are detected for each condition are given in Tab. 5.4 and corresponding exemplary microscopy images are shown in Fig. 5.18 , e.g., 1960 filaments are considered in the red box at $4 \mathrm{~h}$ of assembly time, representing the $20 \mathrm{mM}$ PBA concentration. Further data sets are acquired and the results are given in the appendix A. Vimentin incubated with no PBA for the observed time points is shown blue, whereas the green colour coded boxes refer to $5 \mathrm{mM}$ of PBA during incubation. Filament lengths analysed from the 


\section{\begin{tabular}{l|l} 
Chapter 5 & RESULTS
\end{tabular}}

a)

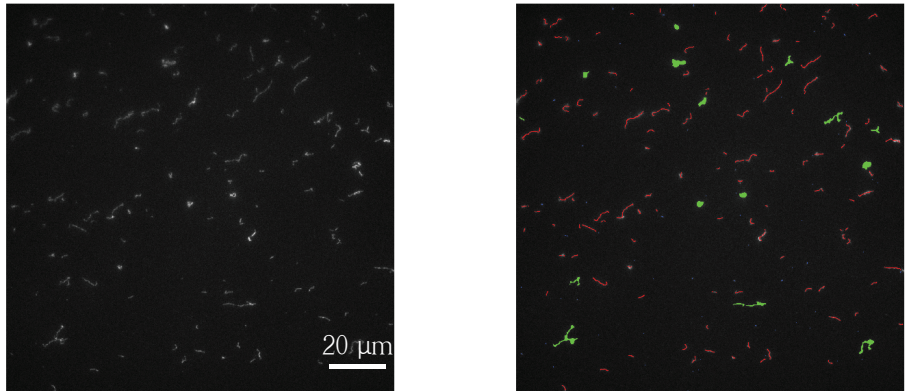

0 mM PBA, 100 mM NaCl, 4 hours assembly

b)

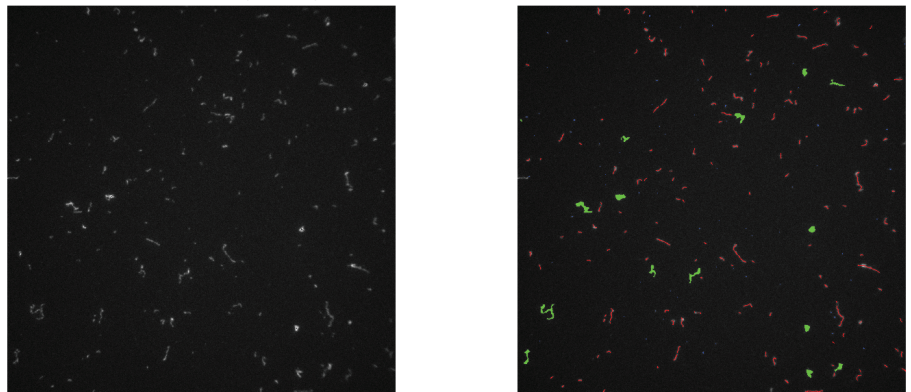

5 mM PBA, 100 mM NaCl, 4 hours assembly

C)

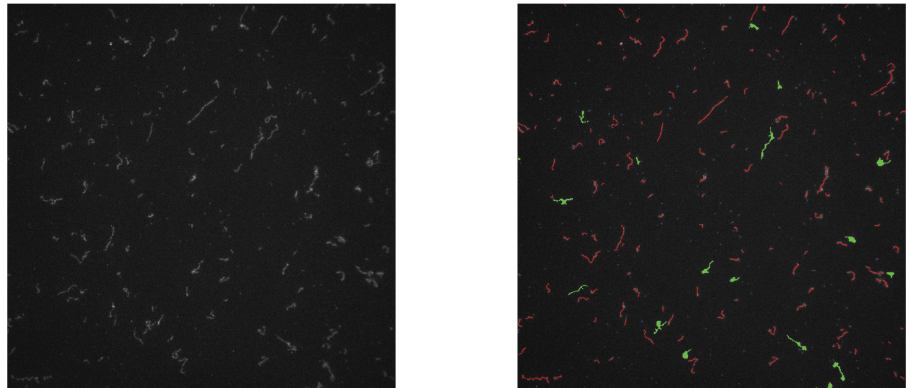

20 mM PBA, 100 mM NaCl, 4 hours assembly

d)
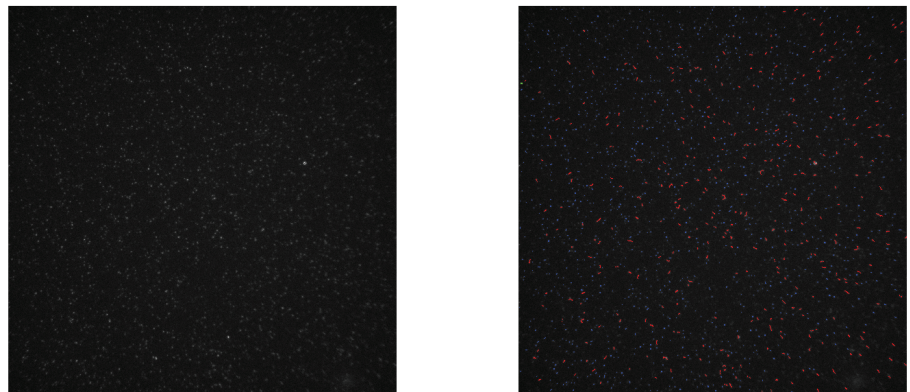

50 mM PBA, 100 mM NaCl, 4 hours assembly

Figure 5.17.: Epi-fluorescent images of vimentin after 4 hours at $37{ }^{\circ} \mathrm{C}$ in $5 \mathrm{mM}$ TRIS with $100 \mathrm{mM} \mathrm{NaCl}$ and varying concentration of PBA (left side). Results of the structures detected by the detection algorithm are shown on the right. Filaments are shown in red and aggregates in green. Concentration of PBA increases from top to bottom, with a) containing $0 \mathrm{mM}$ PBA, b) $5 \mathrm{mM}$ PBA., c) $20 \mathrm{mM}$ PBA. and d) $50 \mathrm{mM}$ PBA. 


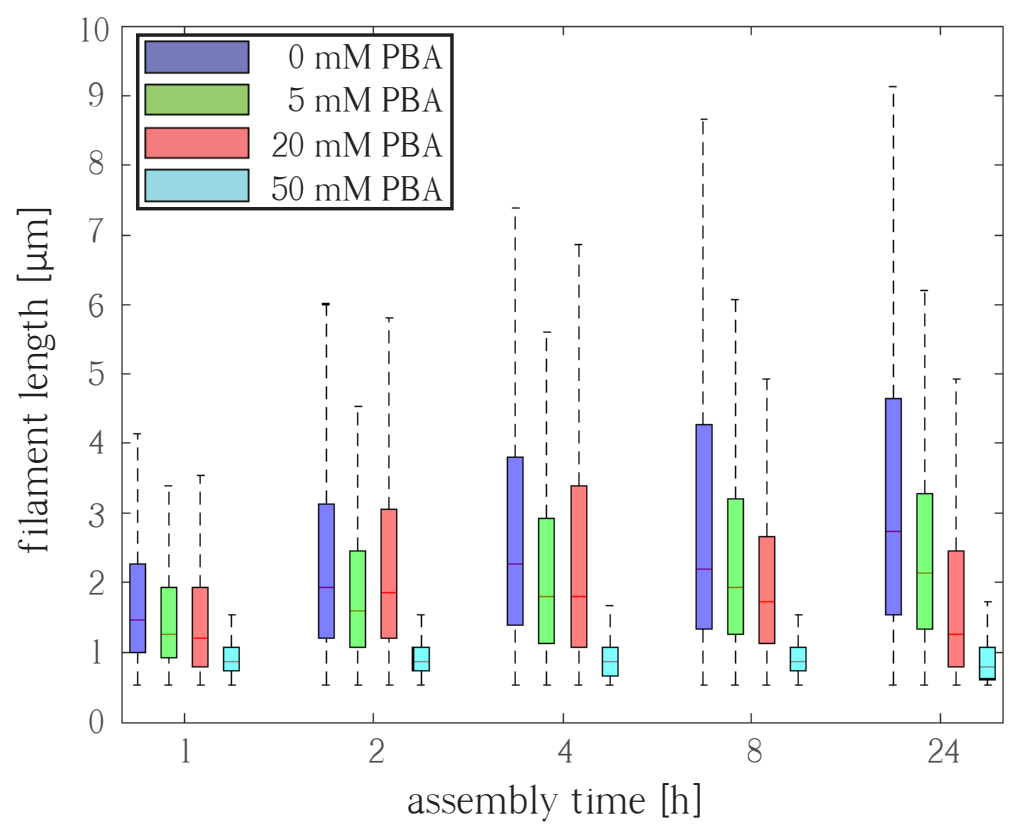

Figure 5.18.: Length distributions of vimentin tetramers incubated with different PBA concentrations over $1 \mathrm{~h}, 2 \mathrm{~h}, 4 \mathrm{~h}, 8 \mathrm{~h}$, and $24 \mathrm{~h}$. For each time point the related length distribution in $\mu \mathrm{m}$ is box plotted, the central mark indicating the median, and bottom and top edges of the box indicating the 25th and 75th percentiles, respectively. $0 \mathrm{mM}$ PBA is shown in blue, $5 \mathrm{mM}$ PBA in green, $20 \mathrm{mM}$ PBA in red and $50 \mathrm{mM}$ PBA in turquoise. The number of filaments for each box is shown in Tab. 5.4.

Table 5.4.: Number of filaments detected for the different PBA concentration for each time point. The number includes filaments from multiple images taken for the same condition, e.g., 2142 filaments for $0 \mathrm{mM} \mathrm{ACC}$ after $4 \mathrm{~h}$ of assembly. Length distribution in $\mu \mathrm{m}$ for each condition are shown in Fig. 5.18.

\begin{tabular}{|l|l|r|r|r|r|}
\hline & \multicolumn{5}{|c|}{ PBA concentration } \\
\hline & & $0 \mathrm{mM}$ & $5 \mathrm{mM}$ & $20 \mathrm{mM}$ & $50 \mathrm{mM}$ \\
\cline { 2 - 6 } assembly time & 1 hour & 2655 & 2102 & 1135 & 1359 \\
\cline { 2 - 6 } & 2 hours & 1529 & 2363 & 1499 & 1384 \\
\cline { 2 - 6 } & 4 hours & 2142 & 2540 & 1960 & 3090 \\
\cline { 2 - 6 } & 8 hours & 1233 & 1808 & 1087 & 1233 \\
\cline { 2 - 6 } & 24 hours & 822 & 1281 & 795 & 1473 \\
\hline
\end{tabular}




\section{\begin{tabular}{l|l} 
Chapter 5 & RESULTS
\end{tabular}}

incubation with $20 \mathrm{mM}$ PBA is depicted in red and the highest concentration of 50 $\mathrm{mM}$ PBA during the incubation is shown in blue. For $0 \mathrm{mM}$ of PBA the filament length increases over time monotonously, as no interaction with PBA can occur and the vimentin continues to assemble into longer filaments during incubation. Furthermore, the lengths measured for $0 \mathrm{mM}$ PBA are the longest for each time point. The sample containing $5 \mathrm{mM}$ PBA contains longer filaments after longer incubation times, as the median for $5 \mathrm{mM}$ PBA, monotonously increases over time. Measured lengths are smaller compared to the control group with no PBA for all time points. Compared to the next higher concentration of PBA, the $20 \mathrm{mM}$ PBA sample, the lengths are longer for incubation times of $8 \mathrm{~h}$ and $24 \mathrm{~h}$, equal to $1 \mathrm{~h}$ and shorter for $2 \mathrm{~h}$ and $4 \mathrm{~h}$. The highest concentration of PBA with $50 \mathrm{mM}$ contains with filament lengths below $1 \mu \mathrm{m}$ for all measured time points the shortest lengths compared to the other PBA concentrations.

\subsubsection{Aggregate Distributions}

Additional to the length distributions described in the previous section, the aggregate size distributions in pixel are calculated. One pixel corresponds to $66 \mathrm{~nm}$ $\times 66 \mathrm{~nm}$. Fig. 5.19 shows the distributions ordered by PBA concentration and incubation time. A total of 3671 aggregates are detected. Details about how many aggregates are found for each conditions are given in Tab. 5.5.

Vimentin incubated without PBA (blue) shows an increase in both aggregate size and number for increasing assembly times. This is as well as the monotonous increase in length, shown in Fig. 5.18 expected, as filament lengths increases over time and thus the aggregates of coiled or filaments with loops increase in size. One exception to this expected trend is the data collected for $4 \mathrm{~h}$ of assembly time. The median aggregate size is with 1253 pixels higher than the median aggregate size measured at $8 \mathrm{~h}$ with 1070 pixels. For $5 \mathrm{mM}$ PBA a slight increasing trend in aggregate size is visible, too. The increase is smaller than for $0 \mathrm{mM}$ PBA, but is consistent with the slight increase in filament lengths, shown in Fig. 5.18. The time-dependent medians of the aggregate size distributions for $20 \mathrm{mM}$ PBA, shown in red, are relative constant over time, with 860 pixels for $1 \mathrm{~h}, 822$ pixels for $2 \mathrm{~h}, 977$ pixels for $4 \mathrm{~h}, 850$ pixels for $8 \mathrm{~h}$ and 1280 pixels for $24 \mathrm{~h}$. A slight increase for the $4 \mathrm{~h}$ and $24 \mathrm{~h}$ measurements is visible. For incubation times of $1 \mathrm{~h}$ the traced aggregates are larger than the control group. Supporting this observation are the 25th and 75th percentiles and the whiskers, showing similar range and size for $1 \mathrm{~h}, 2 \mathrm{~h}$ and $4 \mathrm{~h}$. The sample containing $50 \mathrm{mM}$, depicted in turquoise, shows 


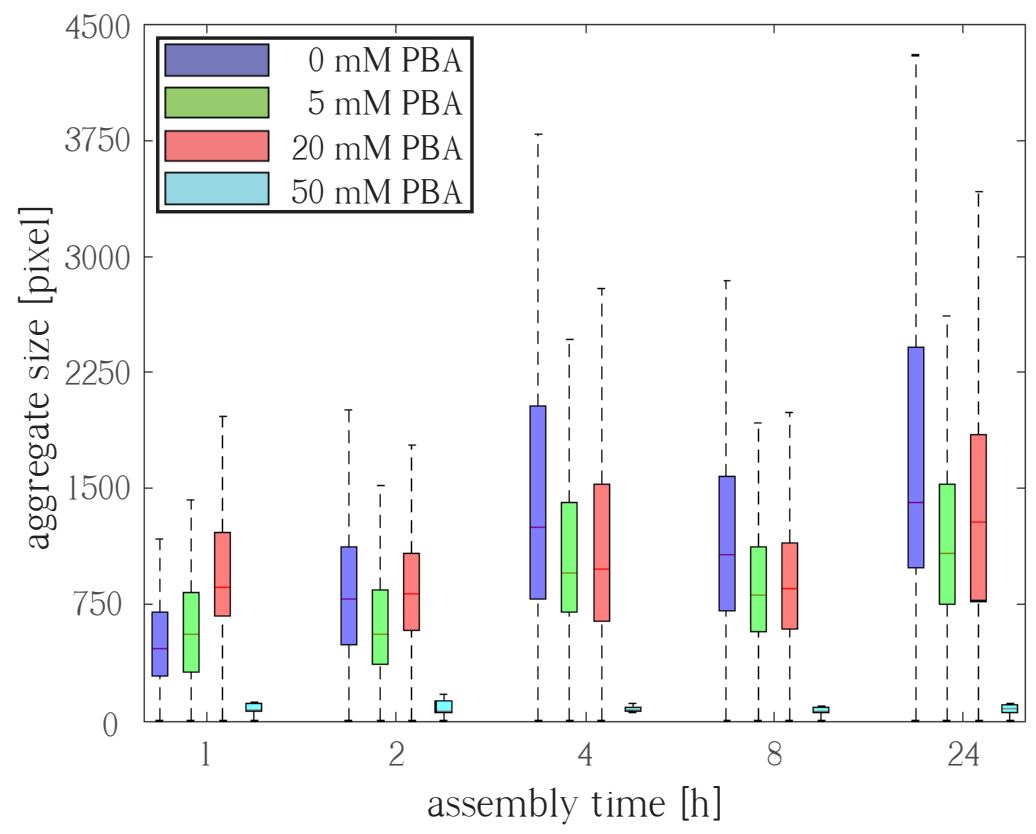

Figure 5.19.: Aggregate distributions of vimentin tetramers incubated with different PBA concentrations over $1 \mathrm{~h}, 2 \mathrm{~h}, 4 \mathrm{~h}, 8 \mathrm{~h}$, and $24 \mathrm{~h}$. For each time point the related aggregate distribution in pixel is box plotted, the central mark indicating the median, and bottom and top edges of the box indicating the 25 th and 75 th percentiles, respectively. $0 \mathrm{mM}$ PBA is shown in blue, $5 \mathrm{mM}$ PBA in green, $20 \mathrm{mM}$ PBA in red and $50 \mathrm{mM}$ PBA in turquoise. The number of aggregates for each data point is shown in Tab. 5.5.

Table 5.5.: Number of aggregates detected for the different PBA concentration for each time point. The number includes aggregates from multiple images taken for the same condition, e.g. 43 aggregates for $20 \mathrm{mM} \mathrm{ACC}$ after $4 \mathrm{~h}$ of assembly. Aggregate size distribution in pixels for each condition are shown in Fig. 5.19.

\begin{tabular}{|l|l|r|r|r|r|}
\hline & \multicolumn{5}{|c|}{ PBA concentration } \\
\hline \multirow{4}{*}{ assembly time } & & $0 \mathrm{mM}$ & $5 \mathrm{mM}$ & $20 \mathrm{mM}$ & $50 \mathrm{mM}$ \\
\cline { 2 - 6 } & 1 hour & 108 & 80 & 118 & 17 \\
\cline { 2 - 6 } & 2 hours & 167 & 166 & 165 & 13 \\
\cline { 2 - 6 } & 4 hours & 479 & 398 & 353 & 28 \\
\cline { 2 - 6 } & 8 hours & 290 & 282 & 188 & 14 \\
\cline { 2 - 6 } & 24 hours & 301 & 302 & 189 & 13 \\
\hline
\end{tabular}




\section{\begin{tabular}{l|l} 
Chapter 5 & RESULTS
\end{tabular}}

the smallest amount of aggregates for all 5 given time points (17 aggregates after $1 \mathrm{~h}, 13$ aggregates after $2 \mathrm{~h}, 28$ aggregates after $4 \mathrm{~h}, 14$ aggregates after $8 \mathrm{~h}$ and 13 aggregates after $24 \mathrm{~h}$ ). This correlates with the microscopy images, where no aggregates are visible for this condition. Furthermore, the amount of aggregates and their size below 150 pixels is stable over the time. Combined with the stable length distributions below $1 \mu \mathrm{m}$ (see Fig. 5.18 turquoise boxes), it becomes clear, that $50 \mathrm{mM}$ PBA complete inhibits vimentin assembly.

\subsubsection{Molecular Mass and Radius of Gyration}

In-house SAXS experiments are performed on vimentin incubated with PBA at 0 $\mathrm{mM}, 5 \mathrm{mM}, 20 \mathrm{mM}$ and $50 \mathrm{mM}$. In Fig. 5.20 the scattering profiles for vimentin assembled with PBA are shown for 3 different measurements. For $0 \mathrm{mM} \mathrm{PBA,}$ $5 \mathrm{mM}$ PBA and $20 \mathrm{mM}$ the scattering profiles look similar, concerning slope and projected $I_{0}$. For $20 \mathrm{mM}$ a slight increase in steepness as well as a larger projected $I_{0}$ are visible qualitatively. For $50 \mathrm{mM}$ PBA this change is further pronounced. Results of the Guinier analysis are shown in Fig. 5.21a. Figure 5.21a shows an increase in $R_{G}$ for $20 \mathrm{mM}$ PBA and $50 \mathrm{mM}$ PBA for each data set. The mean $\overline{R_{G}}$ for $0 \mathrm{mM} \mathrm{PBA}$ is $5.38 \mathrm{~nm}$, for $5 \mathrm{mM} \mathrm{PBA} \overline{R_{G}}=5.31 \mathrm{~nm}$, for $20 \mathrm{mM} \overline{R_{G}}=7.17 \mathrm{~nm}$ and for $50 \mathrm{mM} \mathrm{PBA} \overline{R_{G}}=11.6 \mathrm{~nm}$. Furthermore, the slope of the scattering profile for $50 \mathrm{mM}$ PBA steepens. This indicates an increase in the lateral radius. The increase in structure size is also visible in the projected $I_{0}$, shown in Fig 5.21b. For $20 \mathrm{mM}$ PBA $I_{0}$ increases from 0.019 a.u. for $5 \mathrm{mM}$ PBA to 0.025 a.u.. The highest $I_{0}$ is calculated for $50 \mathrm{mM}$ PBA with 0.052 a.u.. 


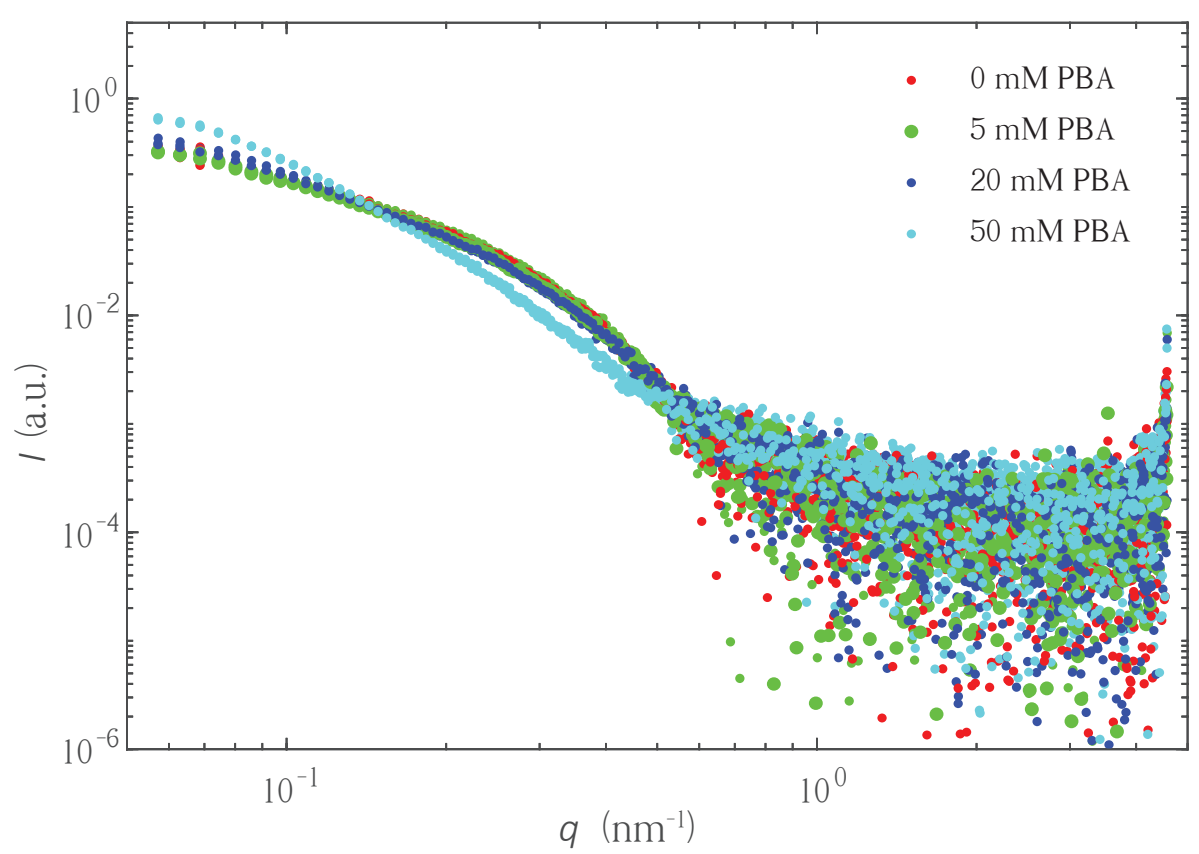

Figure 5.20.: Scattering profiles of vimentin assembled with various PBA concentrations. PBA concentrations range from $0 \mathrm{mM}$ PBA over $5 \mathrm{mM}$ and $20 \mathrm{mM}$ to $50 \mathrm{mM}$. For each concentration 3 independent measurements are shown. Scattering profiles for $0 \mathrm{mM}$ PBA and $5 \mathrm{mM}$ PBA look similar, whereas for $20 \mathrm{mM}$ the steepness and the projected $I_{0}$ increases. For $50 \mathrm{mM}$ PBA both the slope and the projected $I_{0}$ increase even further.
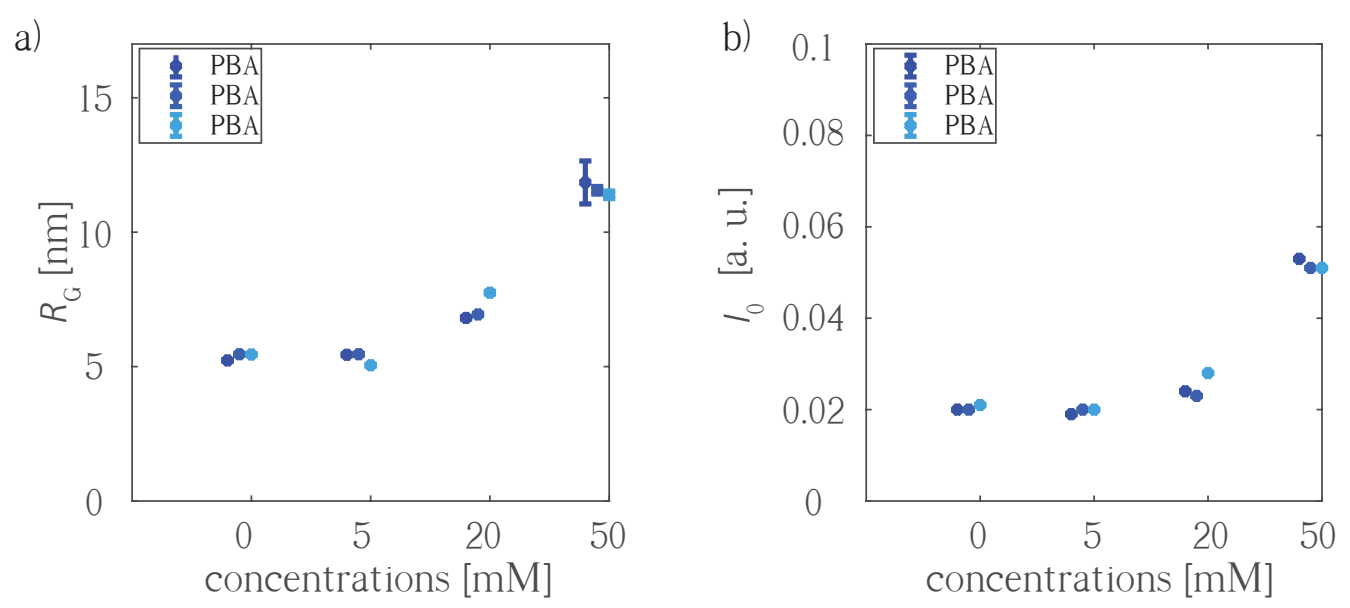

Figure 5.21.: Guinier analysis for the scattering profiles shown in Fig. 5.20. a) The radius of gyration is calculated and shown for each PBA concentration ranging from $0 \mathrm{mM}$ over $5 \mathrm{mM}$ and $20 \mathrm{mM}$ to $50 \mathrm{mM}$. The different data sets are colour coded, with one colour showing the $R_{G}$ for the different conditions. An increase of $R_{G}$ for $20 \mathrm{mM} \mathrm{PBA}$ and $50 \mathrm{mM}$ PBA concentrations is visible. b) Calculated forward scattering intensity $I_{0}$ for the colour coded 3 different data sets. For $20 \mathrm{mM}$ PBA and $50 \mathrm{mM}$ PBA concentrations the calculated $I_{0}$ increases. 
5.3.4 Comparison of the Vimentin Assembly in the Presence of $\mathrm{N}$ Acetyl-L-Cysteine and Phenylbutyrate

\subsubsection{Fluorescence Microscopy Data}

The vimentin length distributions obtained for the control measurements, thus $0 \mathrm{mM}$ ACC or PBA, are in good agreement with values previously reported results. Winheim et al. measured the length of vimentin filaments assembled for two hours, using total internal reflection fluorescence microscopy, and reported lengths between 2 - $4 \mu \mathrm{m}$ [20]. For similar conditions, values between $1.3-3.1 \mu \mathrm{m}$, representing the 25th and 75th percentiles, respectively (see Fig. 5.18), are found within this thesis. Using electron microscopy, Mücke et al. reported a contour length recorded with electron microscopy of 0.6 to $1.6 \mu \mathrm{m}$ for vimentin filaments assembled in similar conditions for twenty minutes to one hour [107]. This correlates to the 25th and 75th percentiles of the detected length distributions after 1 hour of $1-2.2 \mu \mathrm{m}$, respectively (see Fig. 5.18).

For longer assembly times Winheim et al. report filaments lengths between 4 $15 \mu \mathrm{m}$ after 4 hours of assembly. In this thesis filament lengths of $1.5-4 \mu \mathrm{m}$ are detected after assembly for the identical time for the 25th and 75th percentiles, respectively. Although single longer filaments with lengths up to $7.5 \mu \mathrm{m}$ are detected, the overall detected length is shorter than the length reported by Winheim et al.. An explanation for the non-identification of longer filaments, visible in the microscopy images (see Fig. 5.17), is found in the frequent forming of filament loops when filaments attach to the glass surface. The algorithm is unable to properly follow the filament through intersections and thus specifies the structure as an aggregate. Therefore, to quantify the increase in length of the filament, both the length and the aggregate size have to be taken into account.

In addition to the control group the filament lengths and aggregate sizes for ACC and PBA vary for different conditions, indicating different interactions occurring between ACC and PBA and the vimentin tetramers during assembly. Two exemplary data sets, one each for ACC and PBA, are described in detail in Sec. 5.3.2.1 and Sec. 5.3.3.1 for the length distributions and in Sec. 5.3.2.2 and Sec. 5.3.3.2 for the aggregate distributions. To facilitate comparison, both data sets are combined in Fig. 5.22a+b for the detected length and aggregate distributions, respectively. Concentrations of ACC and PBA are colour coded, with $0 \mathrm{mM}$ shown 

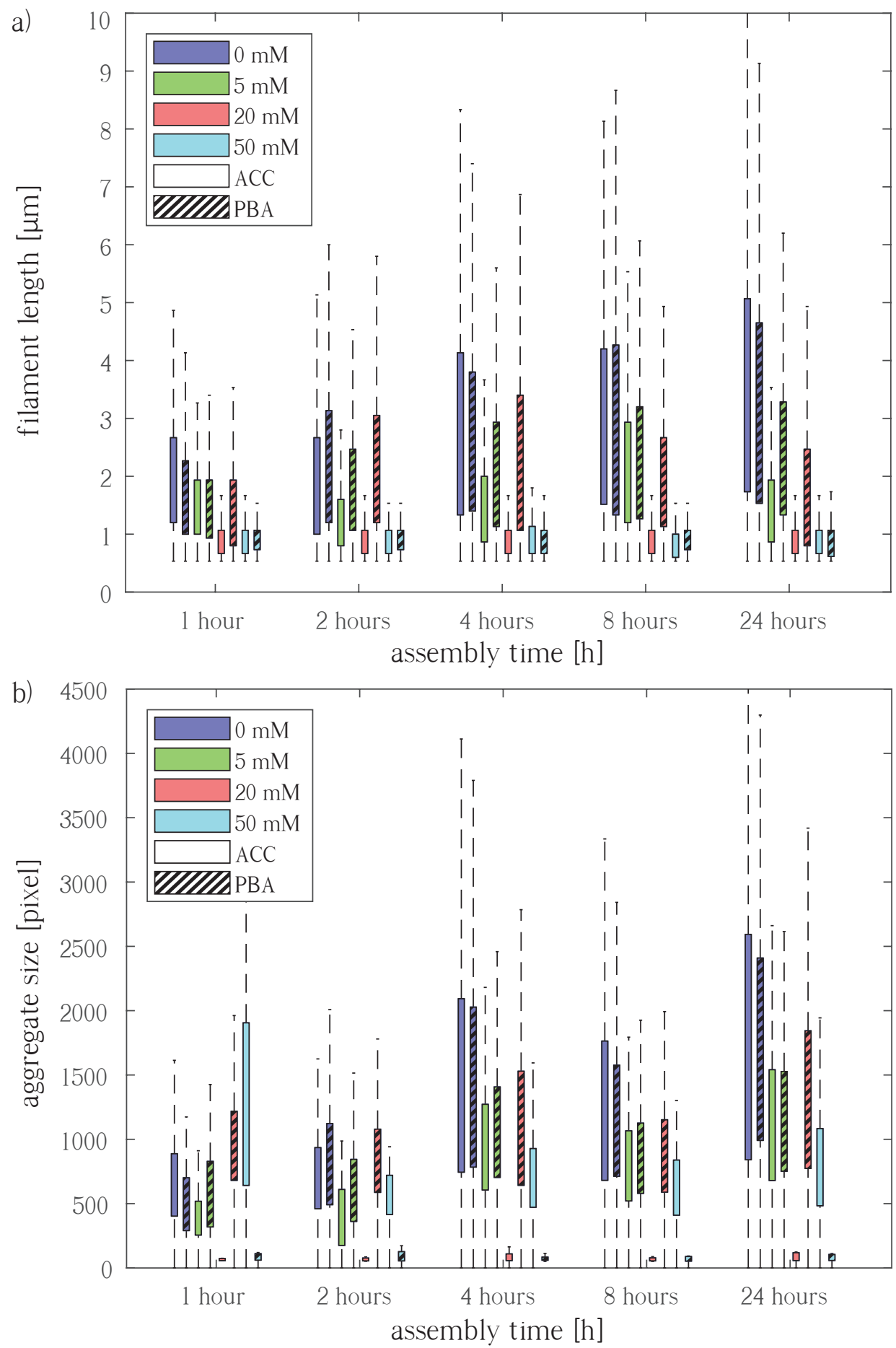

Figure 5.22.: a) Length and b) aggregate distributions of vimentin tetramers incubated with different PBA and ACC concentrations over $1 \mathrm{~h}, 2 \mathrm{~h}, 4 \mathrm{~h}, 8 \mathrm{~h}$, and $24 \mathrm{~h}$. For each time point the central mark indicates the median, and the bottom and top edges the 25th and 75th percentiles, respectively. $0 \mathrm{mM}, 5 \mathrm{mM}, 20 \mathrm{mM}$ and $50 \mathrm{mM}$ concentrations are shown in blue, green, red and turquoise, respectively. Hatched box plots represent PBA data, blank indicates data for ACC. 


\section{\begin{tabular}{l|l} 
Chapter 5 & RESULTS
\end{tabular}}

in blue, $5 \mathrm{mM}$ in green, $20 \mathrm{mM}$ in red and $50 \mathrm{mM}$ in turquoise. Hatched entries correspond to PBA concentrations, whereas non-hatched entries correspond to ACC concentrations. To facilitate comparison between identical concentrations and assembly times, the corresponding ACC and PBA distributions are shown next to each other. Note, that besides the PBA and ACC concentrations, all conditions, e.g., $\mathrm{NaCl}$ concentration, temperature and the $\mathrm{pH}$ value are kept constant (see Sec. 4.1.3 for details).

The length distributions of both control groups (Fig. 5.22a, blue boxes) increase systematically over time for longest detected filaments, indicated by the whiskers, and overall detected length, indicated by the 25th and 75th percentiles, thus verifying no difference of the vimentin assembly without ACC or PBA. This observation is confirmed by identical behaviour of the detected aggregate sizes (Fig. 5.22b, blue boxes). Note the relative stronger ( $\approx 3$ times) increase of the aggregate distributions between 1 hour and 24 hours of assembly compared to the filament lengths $(\approx 2$ times). These relative increases explain the described difference in detected and by Winheim et al. reported filament lengths for longer assembly times [20].

The data for the addition of $5 \mathrm{mM}$ ACC or PBA is displayed by the green boxes. With respect to the control group both the lengths and aggregates the detected structure sizes are reduced, thus inhibition of the vimentin assembly occurs. However, with respect to only the $5 \mathrm{mM}$, the filament lengths and aggregate sizes increase over time. Interestingly, ACC inhibits the vimentin assembly stronger, resulting in shorter filaments and smaller aggregates for all observed time points with concentrations smaller than $50 \mathrm{mM}$. Although both chemical compounds inhibit the vimentin assembly, they both increase the filament length and aggregates sizes over time. The observations are true for all cases where no complete inhibition of filament or aggregate formation occurs.

By increasing the concentration of ACC and PBA from $5 \mathrm{mM}$ to $20 \mathrm{mM}$, the first major difference occurs (see Fig. 5.22a+b, red boxes). Aggregates clearly identifiable as coiled vimentin are in the following referred to as functional aggregates, whereas aggregates without clearly identifiable coiled vimentin are referred to as non-functional aggregates. Distinction between both cases is performed by visual inspection of the corresponding fluorescence images.

ACC prohibits vimentin to form filaments or functional aggregates. This is 
manifested by the short and compact filament sizes, later indicated by the 25th and 75th percentiles, just above the filtering threshold of small objects. The inhibition is even more visible for the aggregate size distributions. In addition, the inhibition is independent of the assembly time, as no change in aggregate size or filament length occurs over time. For PBA little difference in the detected lengths occurs with respect to $5 \mathrm{mM}$. Regarding the aggregate sizes, even a slight increase in the 75th percentile is visible with increasing assembly time. Thus, the vimentin can assemble to larger functional aggregates. These observations are further visible in the microscopy images, exemplary shown for 4 hours assembly in Fig. 5.11 for ACC and Fig. 5.17 for PBA. Whereas for $20 \mathrm{mM}$ PBA no clear difference is observable, as neither filaments nor aggregates are visible for $20 \mathrm{mM}$ ACC.

Applying the highest concentration of $50 \mathrm{mM}$ (see Fig. 5.22a+b, turquoise boxes) for PBA and ACC results in no further change in the filament formation in the presence of ACC. No formed filaments are observed. Interestingly, the filament length for PBA, as well as the aggregate sizes change for both the ACC and the PBA. For $50 \mathrm{mM}$ PBA no longer filaments or aggregates are visible in the microscopy images or detectable, thus inhibition of vimentin assembly occurs. Surprisingly, for the $50 \mathrm{mM}$ of ACC vimentin aggregates form. Note, that these aggregates are non-functional aggregates, as no filament formation but only aggregation occurs. These observations hold for all observed time points, as no variation between different assembly times is observable. Note, that the detected aggregate sizes for $50 \mathrm{mM}$ ACC after 1 hour of assembly contain artifacts, increasing the aggregate size distribution, as described in Sec. 5.3.2.2. 


\section{\begin{tabular}{l|l} 
Chapter 5 & RESULTS
\end{tabular}}

\subsubsection{Static Small-Angle X-Ray Scattering}

To facilitate the comparison between the recorded data on vimentin assembled with PBA and ACC, Fig. 5.23 summarises the results shown in Fig. 5.21 and Fig. 5.16. The applied concentrations of ACC (see Fig. 5.23a) and PBA (see Fig. 5.23b) during the assembly process are colour coded, with $0 \mathrm{mM}$ concentration shown in blue, $5 \mathrm{mM}$ in green, $20 \mathrm{mM}$ in red and $50 \mathrm{mM}$ in turquoise. No data is shown for the addition $50 \mathrm{mM}$ ACC during assembly, as explained in Sec. 5.3.2.3.

For all data sets the calculated mean radii of gyration for the control groups of $\overline{R_{G}}=5.38 \mathrm{~nm}$ and $\overline{R_{G}}=5.33 \mathrm{~nm}$ for PBA and ACC, respectively, are comparable to reported radii of assembled vimentin [16, 22, 108], e.g., Brennich et al. reported a radius of $5.5 \mathrm{~nm}$ for vimentin assembled with $100 \mathrm{mM} \mathrm{KCl} \mathrm{[24].}$

For both compounds no difference in $R_{G}$ or $I_{0}$ is measured for $5 \mathrm{mM}$ of either ACC or PBA when compared to the control group of $0 \mathrm{mM}$. Compared to the control group, $20 \mathrm{mM}$ of both $\mathrm{ACC}$ and PBA resulted in an increase in $\overline{R_{G}}$ of the vimentin in both groups. This increase is more pronounced for PBA with $\overline{R_{G}}=7.17 \mathrm{~nm}$ than it is for ACC with $\overline{R_{G}}=6.08 \mathrm{~nm}$. For $50 \mathrm{mM}$ PBA a large increase in both the radius of gyration and the forward scattering intensity is measured. $\overline{R_{G}}$ increases from $7.17 \mathrm{~nm}$ to $11.6 \mathrm{~nm}$ and $\overline{I_{0}}$ increases from 0.025 [a.u.] to 0.052 [a.u.]. Although no exact molecular mass can be calculated, the increase of $\overline{I_{0}}$ for PBA indicates an increase of the molecular mass compared to the control. Combined with the corresponding scattering profile (see Fig. 5.20), which show a change in
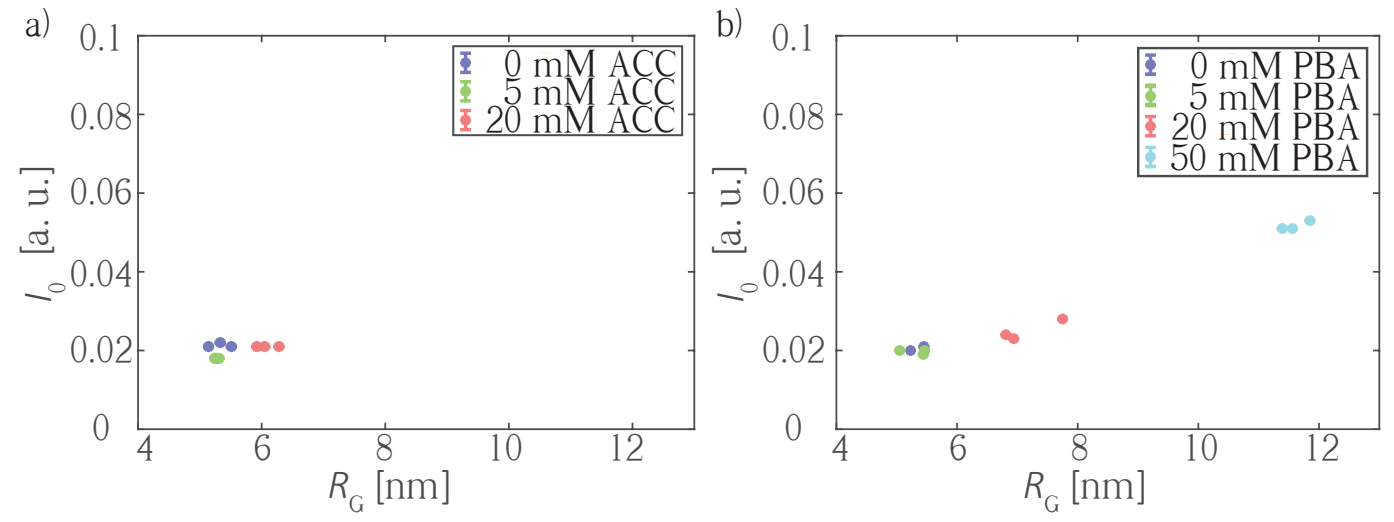

Figure 5.23.: Summarized Guinier analysis for a) ACC and b) PBA, shown in Fig. 5.21 and Fig. 5.16, respectively. The radius of gyration $R_{G}$ is plotted against the calculated forward scattering intensity $I_{0}$ for the vimentin assembly with various concentrations of ACC and PBA. Concentrations employed are colour coded with $0 \mathrm{mM}$ in blue, $5 \mathrm{mM}$ in green, $20 \mathrm{mM}$ in red and $50 \mathrm{mM}$ in turquoise. For each condition three different data sets are shown. 
the slope for this condition, a change in the radius appears to also occur. This is further supported by the increased $\overline{R_{G}}$ of $11.6 \mathrm{~nm}$. For $50 \mathrm{mM}$ ACC no $\overline{R_{G}}$ or $\overline{I_{0}}$ for comparison could be calculated, as the scattering intensity profile indicates aggregation of the sample.

By combining SAXS and the microscopy images and the results from the filament detector algorithm the following general behaviours of ACC and PBA influencing vimentin assembly can be concluded. Both chemical compounds inhibit the formation of vimentin intermediate filaments. With increasing concentration the inhibition intensifies, until no filaments remain. This critical concentration is $20 \mathrm{mM}$ for ACC and $50 \mathrm{mM}$ for PBA (see Fig. 5.22a). Besides the filament length directly calculated, filaments bundled up in functional aggregates, thus no clear filament length can be distinguished. The size of these functional aggregates is reduced simultaneously with the length for increased concentrations of ACC and PBA and shares the identical critical concentrations, where no longer aggregates occur, for both chemical compounds (see Fig. 5.22b). For ACC no change in the molecular mass and only a slight increase in the radius of gyration for the critical concentration of $20 \mathrm{mM}$ is observed from the recorded SAXS data. Interestingly, for an increase of ACC above the critical concentration, non-functional aggregates form, thus no filamentous structures are visible inside the aggregates (see Fig. 5.11). Furthermore, the recorded SAXS data indicate attraction between the scatterers for this conditions (see Fig. 5.14). In contrast to the ACC, the SAXS data for vimentin assembled with PBA indicates an increase in both the radius of gyration and the molecular mass for increases PBA concentrations (see Fig. 5.23). 



\section{Discussion}

In the following, the results described in Sec. 5 are discussed and contextualized with respect to previously reported results. First, the developed microfluidic step devices and the recorded data quality is discussed and a possible explanation for the artifacts occurring during data collection is given. The effects of ACC and PBA on vimentin assembly recorded with the microscopy images, the structure finding algorithm and SAXS are combined and compared. Finally, possible mechanisms of interaction of PBA and ACC with vimentin are proposed.

\subsection{Microfluidic Step Devices for Scanning Small-Angle X- Ray Scattering}

X-ray compatible microfluidic devices are developed and the dynamics of ioninduced vimentin assembly are investigated using time-resolved scanning SAXS (see Sec. 5.2.4). Similar experiments on vimentin assembly and microfluidic devices have already been performed using SAXS [24, 58, 60], fluorescence correlation spectroscopy [104] and light scattering [103] techniques. Regardless of the application, the microfluidic device needs to be adapted to the geometry, material 


\section{\begin{tabular}{l|l} 
Chapter 6 & DISCUSSION
\end{tabular}}

and flowrates.

Although FEM simulations are frequently used in microfluidic studies, e.g., in $[60,104,109]$, the simulation quality is limited by the employed mesh sizes and computational capabilities. Compared to the aforementioned references, here, different regions inside the device are defined with individual mesh sizes for each region: Areas with no relevant diffusion or hydrodynamic focusing activity have a larger mesh size than those that are more relevant (see Fig. 5.2). This approach not only yields results which more-accurately reflect the physical system, but also reduces the necessary computational time of a comparable simulation without defined regions.

Using COC to manufacture X-ray compatible microfluidic devices has been successfully performed for crystallisation of biomolecules [96]. For vimentin assembly non-step microfluidic devices were employed (see Sec. 5.1 [97]), while yielding data quality comparable to well-established multi-material devices such as NOAKapton devices [60]. In addition, COC devices are more mechanically stable than, e.g., NOA-Kapton or Polydimethylsiloxane (PDMS)- quartz glass capillaries devices [109], as no connection between different materials needs to be established. Other approaches for reliably manufacturing mono-material X-ray compatible devices are proposed by van der Linder et al. [110] and Barrett et al. [111]. Van et al. [110] manufactured devices suitable for synchrotron measurements using a 3D printer with precise wavelength selection. Barrett [111] et al. used laser ablation to manufacture thin microfluidic devices in Kapton.

For the COC step-device developed within this thesis, the recorded data is shown in Fig. 5.8. Although an increase in $I_{0}$ is qualitatively visible for longer assembly times, which is expected due to the increase in molecular weight during assembly to ULFs, for ion-induced vimentin assembly this increase occurs typically in the first $100 \mathrm{~ms}$. This time scale is reported from various methods, e.g., light scattering and FCS $[103,104]$. For the data recorded with the COC stepdevice the increase is measured until $867 \mathrm{~ms}$. As this increase cannot origin from the lateral vimentin assembly, this change must be induced by the device itself. As a result, the data quality obtained with the step-devices is poor compared to the data from the non-step devices and is not further analysed. A possible cause for the reduced data quality is discussed in the following.

In the step-devices, most of the recorded scattering patterns contain multiple streaks, often appearing to form a cross pattern. This pattern moves in relation to the measurement position inside the device, as the angle between, and the in- 
tensity of, the streaks changes. By masking the streaks in both the background and sample scattering patterns recorded at a specific scanning location the background correction could be improved, as described in Sec. 5.2.3. Despite masking the streak, the data quality is reduced significantly when compared to non-step COC devices.

A possible explanation of such streaks is the brass master used during the hot embossing step (see Sec. 4.2.1). The brass master is precision milled. However, micrographs from a raster electron microscope (REM) reveal characteristic patterns are milled into the brass master as a direct result of the milling process. Fig 6.1 shows an REM image of milled patterns from the channel section of the microfluidic device. The channel walls are indicated by the red dotted line, whereas
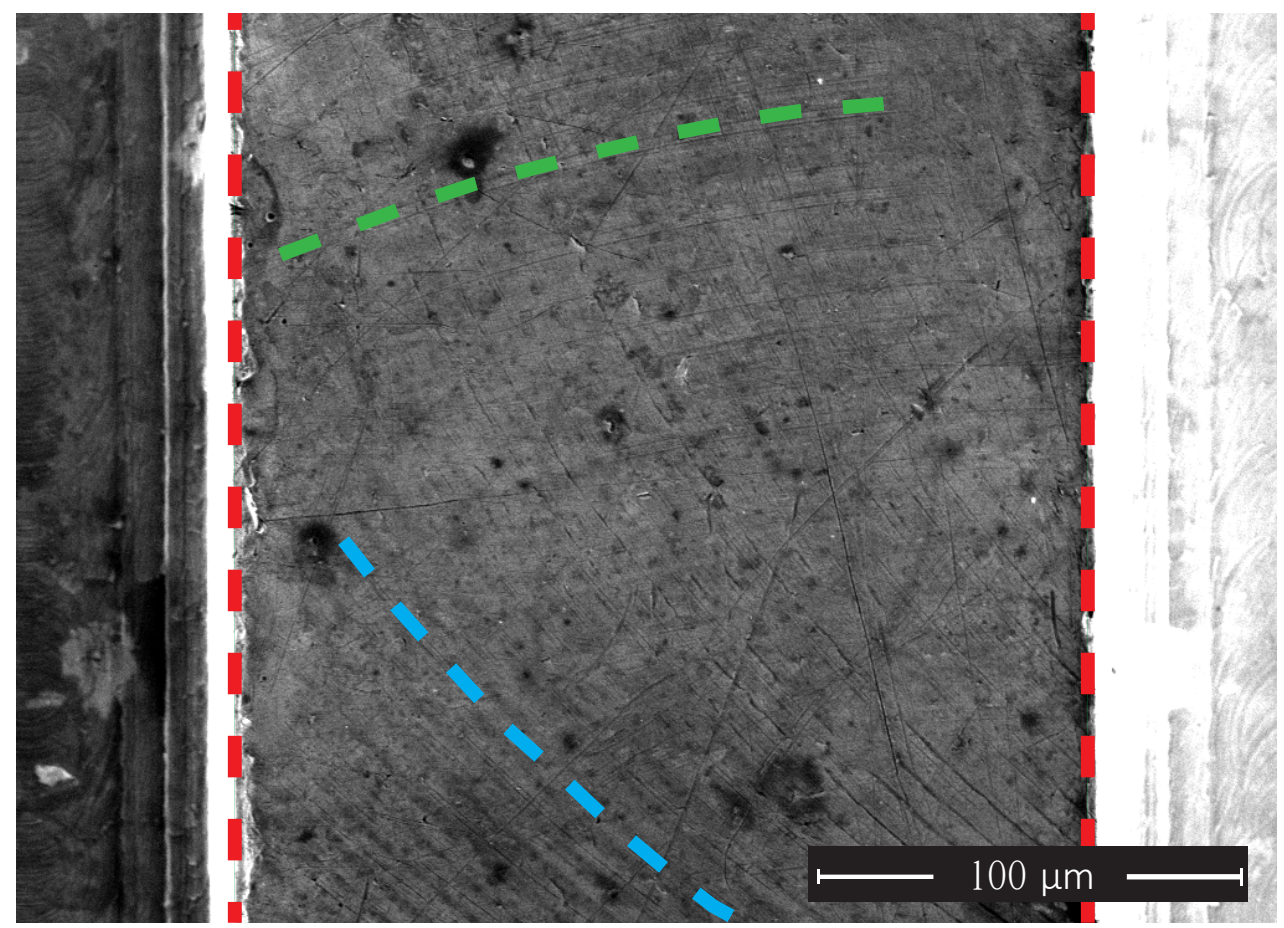

Figure 6.1.: Raster Electron Microscope image of a channel section of the brass master. The channel boundaries are indicated by the red dotted line. Main directions on occurring patterns are indicated by the green and blue dotted line, respectively. REM image recorded with Tobias Meyer (Institut für Materialphysik, Universität Göttingen).

the cyan and green lines follow the main directions of the two observed patterns. During hot embossing these patterns are transferred directly into the COC halves which are later assembled into the step device. The curved patterns explain the changing of the observed angle between the streaks in adjacent scattering pat- 


\section{Chapter 6 DISCUSSION}

terns, as the streaks could be induced by the patterns originating from the milling process. Furthermore, the patterns change their angle with respect to each other, depending on the position inside the channel. This angular change matches the observation from the angles between the streaks in individual scattering patterns.

Two different approaches were tested in an attempt to smoothen the internal surface of the COC device: After the assembly of the two halves the entire device was heated under pressure for additional five minutes at $110{ }^{\circ} \mathrm{C}$. This aimed for the surface tension of the connecting layer of $C O C$, as heated above $T_{G}$, to reduce the patterns by minimizing the surface area.

A second approach follows the protocol established for the non-step devices (see Sec. 5.1 [97]). The channel structures defined by the brass master are replaced by channel structures produced by standard soft lithography methods [112]. These structures possess relatively smoother surfaces. Subsequent moulding with PDMS and NOA 81 results in NOA 81 defining the channel structures needed for hot embossing. Although this process works nicely for non-step devices [97], the step-devices require higher hot embossing temperatures due to higher $T_{G}$ of the structural conserving COC 6013, as described in Sec. 4.2.1. The required temperature for the hot embossing step exceeds the withstanding temperature of NOA 81 [113], resulting in the NOA 81 structures to crumble during the hot embossing. Either approach used to smoothen the inner surface of the COC device was unsuccessful. 


\subsection{Small Molecules Inhibiting Vimentin Assembly}

With the fast advances in high-resolution microscopy more highly dynamic systems are possible to observe. In the context of the cytoskeleton, these systems often are filamentous. To observe these dynamics, e.g., for microtubules, often kymographs are manually created [114]. Kapoor et al. addressed this, by creating a chain tracing algorithm for biological filaments [115]. The filament detector developed in this thesis performs well in detecting the structures in the microscopy images. By automating the detection large amounts of recorded data could be analysed. Whereas the method presented in this thesis is easy to use, as only the input must be selected, more advanced detection methods are recently reported. Schneider et al. recently employed neural networks and spline fitting for the detection of semiflexible polymers in AFM images [116]. The network training is based on test data sets with structures of varying persistence lengths. The authors are confident to be able to employ their algorithm to fluorescent images [116]. Although single filaments are nicely detected, entangled, crossed or looped filaments are still difficult to trace properly. In this thesis all cases of unclear continuity of a filament resulted in the separate detection as 'aggregates'. A different approach is employed by Schneider et al., as connected structures are separated by removing the intersection points. The remaining parts are then detected [116]. A third approach is employed by Kapoor et al. [115]: By representing the traced filament with a polynomial function direction of the individual filament is preserved, thus enabling the tracing of microtubules through intersections [115].

Combining SAXS and optical fluorescence microscopy worked well to investigate the inhibition of ion-induced vimentin assembly by ACC and PBA. For both employed methods the data obtained in the control measurement correlate with data previously reported. Although ACC and PBA both induce an inhibition to vimentin assembly, the mechanism employed appears to vary. This variation occurs in the different critical concentration when neither filaments nor aggregates are visible. Furthermore, the SAXS data reveal an increase in molecular weight and the radius of gyration for increasing concentrations of PBA, whereas for ACC only small changes occur when increasing the concentration. In addition, SAXS data for the highest ACC concentration indicate aggregation, which is complementary, to the microscopy images.

Contextualizing these results in findings reported for several interaction mechanisms and applications of ACC and PBA in severe diseases is performed in the 


\section{\begin{tabular}{l|l} 
Chapter 6 & DISCUSSION
\end{tabular}}

following. Furthermore, specific mechanism of interaction with vimentin during assembly are proposed for both investigated chemical compounds.

\section{Sodium Phenylbutyrate}

PBA is reported to antagonizing protein aggregation in Alzheimer disease, of the type I/II IF keratin and for the type III IF desmin [49-51]. The antagonizing effects of PBA are observed in this work, too: Increasing concentrations of PBA during ion-induced vimentin IF self-assembly reduce the vimentin filament formation in vitro in both filament length and size of functional aggregates. PBA is reported to act as a chaperone, stabilizing proteins with reversible binding, thus inhibiting conformational changes [47, 51].

Based on the radius of gyration, no change in the radius of the formed filament is observed for low concentrations ( $5 \mathrm{mM}$ ) of PBA compared to the control group (see Fig. 5.21a). This correlates with the observed little effect on the filament length and functional aggregates sizes for low concentrations (see Fig. 5.18 and Fig. 5.19). When increasing the PBA concentration the radius of gyration increases, until it becomes doubled when at the largest concentration of $50 \mathrm{mM}$ (see Fig. 5.21). The change in radius is further indicated by the change of the slope in the scattering intensity profile (see Fig. 5.20). Combined with the forward scattering intensity an increase in molecular weight is observed (see Fig. 5.21b), indicating that PBA alters the size of the assembled ULFs. As no further longitudinal assembly occurs, this alteration must prohibit the assembly of ULFs to filamentous structures.

\section{N-Acetyl-L-Cysteine}

Disulphide bond formations occurs during the ion induced self-assembly process of vimentin wild type [28]. These bonds help in conformational changes of the subunit arrangement. Although vimentin IFs can form in the C328S mutant, where no disulphide bonds between neighbouring 328 cysteine can occur, the radius of the resulting filament differs from assembled of vimentin wild type filaments [29]. The importance of disulphide bonds during vimentin IF self-assembly in vitro is further emphasized, by electrophilic compounds, such as diamide. The increased disulphide bond formation severely impairs the assembly of vimentin to ULF and, ultimately, leads to the formation of non-functional aggregates [29].

ACC is well known for its antioxidant and disulphide bond breaking capabilities. By binding with its own thiolate group to a thiol group it forms a disulphide 
bond, inhibiting further bonding of other thiol groups. This enables ACC to disassemble aggregated proteins, e.g., mucin in cystic fibrosis [52]. This mechanism could furthermore apply for vimentin IF during ion-induced self-assembly.

From the SAXS data presented in this work, no change in radius of gyration or molecular mass is measured for increased ACC concentrations compared to vimentin assembled without ACC. This indicates that similar ULFs are formed under the influence of ACC. Interestingly, the filament lengths and the size of the functional aggregates of vimentin are reduced for increased ACC concentrations. A possible explanation for this phenomenon could be that the end-to-end annealing, following the ULF formation [16, 20, 21], is antagonized until it can no longer occur. For concentrations above the critical threshold of $20 \mathrm{mM}$ described in Sec. 6.2 non-functional aggregates form. The SAXS data indicates aggregation for this concentration of $50 \mathrm{mM}$ concentration of ACC, thus it is not clear if functional ULFs are formed prior to the non-functional aggregates visible in the fluorescent microscopy images (see Fig. 5.11). 



\section{Conclusions and Future Experiments}

Within this thesis, various methods are employed to investigate different aspects of the vimentin intermediate filament assembly process. Small-angle X-ray scattering provides insights on the occurring lateral assembly on the nanometer scale, and optical fluorescence microscopy provides insight on subsequent longitudinal assembly on the micrometer scale. Microfluidic techniques enable the precise control of chemical conditions and thus reaction times. Combining these techniques enable investigation of ion-induced assembly and small molecules induced assembly inhibition of vimentin intermediate filament.

In the first part of this thesis, early time-points of vimentin intermediate filament assembly are measured by combining microfluidics and small-angle X-ray scattering. Finite element method simulations are first performed to determine suitable flow rates, geometries and ion concentrations for vimentin intermediate filament assembly inside a microfluidic device. Simulation efficiency and results are improved by subdividing the finite element mesh based on theoretical considerations. The mechanical stability and geometry of the microfluidic device is improved by developing manufacturing protocols. Reliability between the fluid connections and microfluidic devices is improved to increase data acquisition effi- 
ciency. Analytic tools are developed, to reduce the impact of device-artifacts. It is shown that the finite element method simulation results are improved without a major increase of computational time by strongly adapting the employed mesh in accordance to occurring dynamics. Reliable manufacturing of microfluidic cyclic olefin copolymers devices is performed using the developed protocols. Dependable fluid connections are established by using the developed device holder. The combination of cyclic olefin copolymers microfluidic devices and small-angle $X-$ ray scattering yields comparable data quality to established device materials, while simultaneously improving the mechanical properties of the microfluidic device. The device geometry was adapted to prevent the sample from interaction with the surface. However, these adaptations resulted in a decrease of data quality.

Combining the finite element method simulations, the device manufacturing and the data acquisition the early time-points of vimentin intermediate filament assembly are accessed with cyclic olefin copolymers microfluidic devices combined with small-angle X-ray scattering. The improvements of the finite element method simulations for the devices employed can be applied to finite element method simulations in general, whereas the device holder may be applied in situations where mechanical stress on the fluid connections occur.

In the second part of this thesis the inhibition of vimentin intermediate filament assembly due to N-Acetyl-L-cysteine and sodium phenylbutyrate is measured by combining optical fluorescence microscopy and small-angle X-ray scattering. A structure finding algorithm is developed to analyse the length of assembled vimentin intermediate filaments. Various N-Acetyl-L-cysteine and sodium phenylbutyrate concentrations and assembly times are investigated, to characterize their effect on assembling vimentin intermediate filaments. Small-angle X-ray scattering data is recorded to gain insights on the occurring effects on lateral assembly. It is shown that the developed algorithm can automatically trace and analyse structures in recorded optical fluorescence images. Automation enables large numbers of images to be processed in a consistent way. Benchmarking with test structures reveals good agreement with expected values. Inhibition of vimentin intermediate filament assembly is shown to be dependent on the N-Acetyl-L-cysteine and sodium phenylbutyrate concentrations and assembly times. A critical concentration is found for N-Acetyl-L-cysteine and sodium phenylbutyrate, where vimentin intermediate filament assembly is completely inhibited. N-Acetyl-L-cysteine and sodium phenylbutyrate induced variations in the lateral assembly are revealed by 
small-angle X-ray scattering, as the molecular mass and the radius of gyration change.

Combining the results from the algorithm-based analysis of the optical fluorescence images and small-angle $\mathrm{X}$-ray scattering, possible mechanisms of interaction of N-Acetyl-L-cysteine and sodium phenylbutyrate with vimentin intermediate filaments are suggested. The understanding of the interactions is important, as both $\mathrm{N}$-Acetyl-L-cysteine and sodium phenylbutyrate are used in modern medicine to treat severe diseases. In a more general approach, the developed algorithm may be employed to analyse large varieties of filamentous structures, enabling further insight in important biophysical processes.

Further experiments could be performed to obtain additional insights in the vimentin intermediate filament assembly inhibition of N-Acetyl-L-cysteine and sodium phenylbutyrate. For both compounds it is reported that preformed structures can be altered [51, 52]. For the case of diamide the induced disassembling effect was only visible in vimentin intermediate filament wild-type protein. When using mutated Cys328Ser the previous occurring effect was negated. Although vimentin intermediate filament assembly occurs with a mutated vimentin Cys328 residue [29], combining the performed experiments with vimentin Cys328Ser could reveal further insights, as N-Acetyl-L-cysteine could interact with the thiol group alike diamide $[45,52]$.

Whereas in this thesis the initial inhibition during assembly is investigated, a similar experiment could be performed after an initial assembly time without $\mathrm{N}$-Acetyl-L-cysteine and sodium phenylbutyrate. Sodium phenylbutyrate shows similarities to heat shock protein 27, which is a chaperone expressed in cells after head-induced cell stress [41]. Heat shock protein 27 interactions with vimentin intermediate filament networks are reported, stabilizing the network and preventing the disassembly despite the induced head stress $[42,43]$. Therefore, it would be interesting to investigate how N-Acetyl-L-cysteine and sodium phenylbutyrate interact with preassembled vimentin intermediate filament.

The small-angle X-ray scattering analysis could be further extended with models including tails, as suggested by Pedersen et al. [77] and applied for vimentin intermediate filament assembly in [117]. The algorithm performance could be further improved by altering the method of spline detection as suggested by Schneider et al. [116]. Combined with methods of handling intersections or loops of filaments, as suggested by Kapoor et al. [115], the variety of applications could be improved. 


\section{\begin{tabular}{l|l} 
Chapter 7 & CONCLUSIONS AND FUTURE EXPERIMENTS
\end{tabular}}

The reduction of signal quality of the further developed cyclic olefin copolymers step-device could be addressed by changing the mould material during the hot embossing step. The protocols developed would presumably still hold, as the manufacturing steps could remain identical. 


\section{A Connecting the Mounted Device and Setting Up the Flow for Microfluidic Scanning Small-Angle X-Ray Scattering Measurements}

1. Cut open the tubing of the outlet

2. Cut open the tubing of one sheath buffer inlet

3. Start the syringe with a speed of $500 \mu \mathrm{L} / \mathrm{h}$

4. Connect the syringe with the tubing (try to get a small air bubble inside, then we know when the liquid of the syringe starts flowing in

5. Once it is connected, increase the speed to $1000 \mu \mathrm{L} / \mathrm{h}$

6. Once the air bubble is near the device, reduce the speed to $50 \mu \mathrm{L} / \mathrm{h}$

7. Start with the second sheath buffer - Redo step 3 - 6 
8. After a few seconds running with the sheath buffers from the syringe, start with one assembly buffer - Same procedure as for the sheath buffers (step 3 - 6) , followed by the 2nd after reducing the speed of the first one

9. Wait 1-2 minutes, then reduce the flow rates of both assembly buffer to 10 $\mu \mathrm{L} / \mathrm{h}$

10. At last, start the protein stream

- Redo step $3-5$

- Once the air bubble is near the device, reduce the speed to $10 \mu \mathrm{L} / \mathrm{h}$

11. Reduce the sheath to $5 \mu \mathrm{L} / \mathrm{h}$ and stop all other flows

Perform background measurement

12. Use the microscope to look at the cross section

13. Set flow rates to: The sheath buffers to $100 \mu \mathrm{L} / \mathrm{h}$, Assembly buffer to 100 $\mu \mathrm{L} / \mathrm{h}$

After the assembly buffer air bubble is gone, or you are sure it is the assembly buffer

14. Increase protein stream to $25 \mu \mathrm{L} / \mathrm{h}$

15. After you see the protein stream: Reduce protein to $10 \mu \mathrm{L} / \mathrm{h}$ and reduce sheath to $50 \mu \mathrm{L} / \mathrm{h}$

16. In the end reduce sheath buffer $(25 \mu \mathrm{L} / \mathrm{h}$ after 1 minute $10 \mu \mathrm{L} / \mathrm{h}$ and in the end $5 \mu \mathrm{L} / \mathrm{h}$ )

17. Let the system stabilize and check for air bubbles

Perform sample measurement 


\section{B Sodium Phenylbutyrate Data Sets}

\section{B.1 Data Set 2}

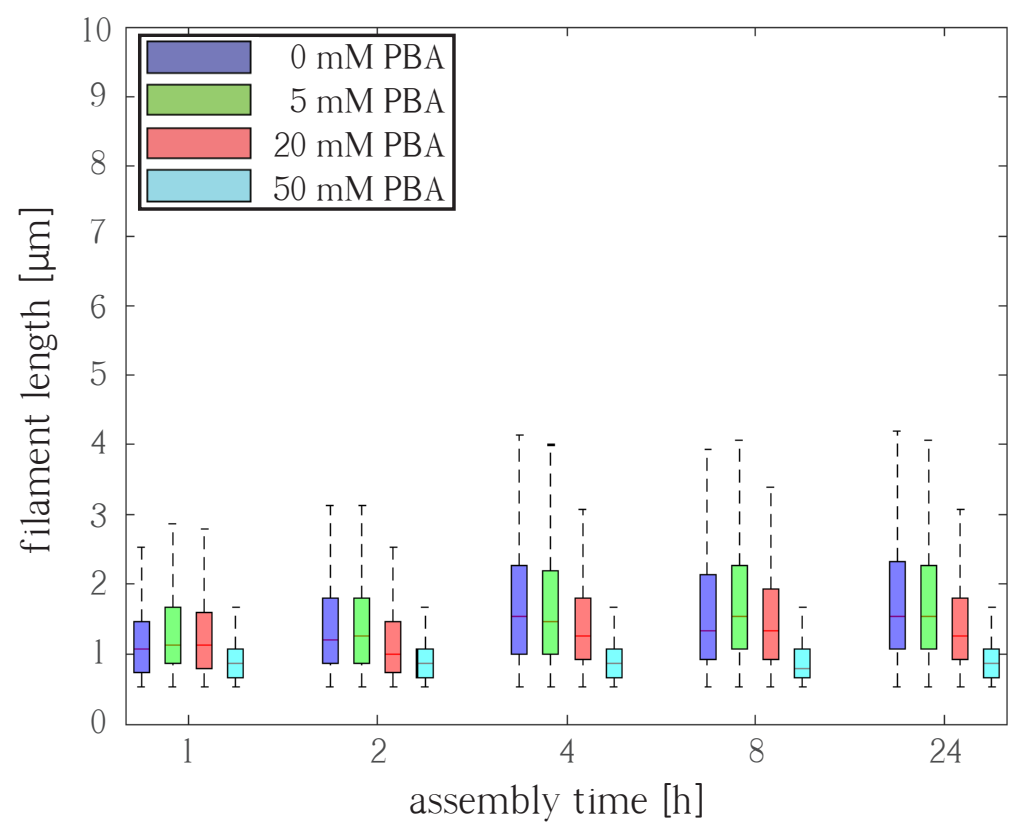

Figure A.1.: Length distributions of vimentin tetramers incubated with different PBA concentrations over $1 \mathrm{~h}, 2 \mathrm{~h}, 4 \mathrm{~h}, 8 \mathrm{~h}$, and $24 \mathrm{~h}$. For each time point the related length distribution in $\mu \mathrm{m}$ is box plotted, the central mark indicating the median, and bottom and top edges of the box indicating the 25th and 75th percentiles, respectively. $0 \mathrm{mM}$ PBA is shown in blue, $5 \mathrm{mM}$ PBA in green, $20 \mathrm{mM}$ PBA in red and $50 \mathrm{mM}$ PBA in turquoise. 


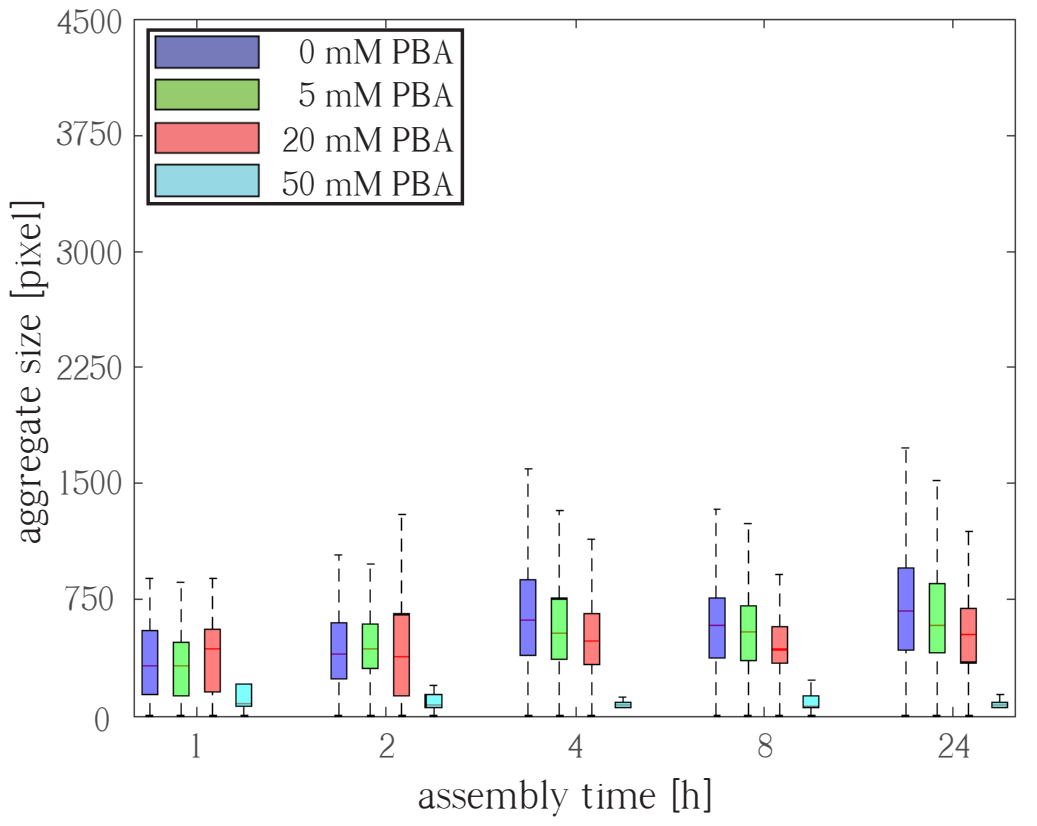

Figure A.2.: Aggregate distributions of vimentin tetramers incubated with different PBA concentrations over $1 \mathrm{~h}, 2 \mathrm{~h}, 4 \mathrm{~h}, 8 \mathrm{~h}$, and $24 \mathrm{~h}$. For each time point the related aggregate distribution in pixel is box plotted, the central mark indicating the median, and bottom and top edges of the box indicating the 25th and 75th percentiles, respectively. $0 \mathrm{mM}$ PBA is shown in blue, $5 \mathrm{mM}$ PBA in green, $20 \mathrm{mM}$ PBA in red and $50 \mathrm{mM}$ PBA in turquoise. 


\section{B.2 Data Set 3}

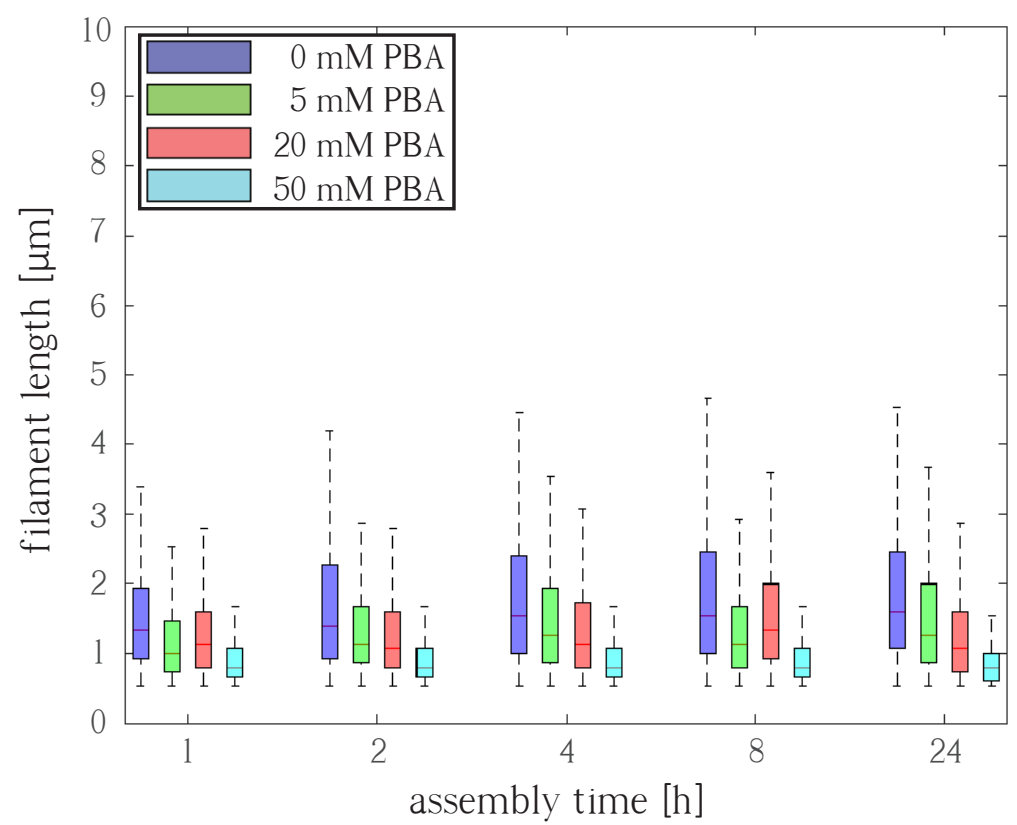

Figure A.3.: Length distributions of vimentin tetramers incubated with different PBA concentrations over $1 \mathrm{~h}, 2 \mathrm{~h}, 4 \mathrm{~h}, 8 \mathrm{~h}$, and $24 \mathrm{~h}$. For each time point the related length distribution in $\mu \mathrm{m}$ is box plotted, the central mark indicating the median, and bottom and top edges of the box indicating the 25th and 75th percentiles, respectively. $0 \mathrm{mM}$ PBA is shown in blue, $5 \mathrm{mM}$ PBA in green, $20 \mathrm{mM}$ PBA in red and $50 \mathrm{mM}$ PBA in turquoise. 


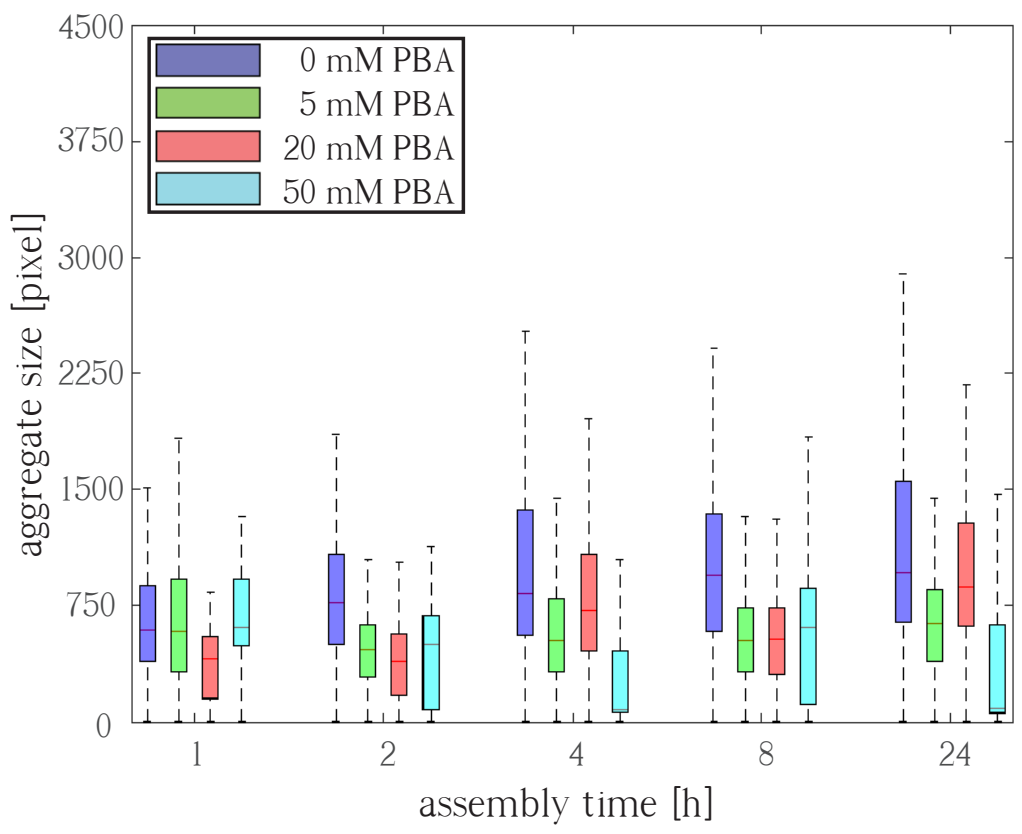

Figure A.4.: Aggregate distributions of vimentin tetramers incubated with different PBA concentrations over $1 \mathrm{~h}, 2 \mathrm{~h}, 4 \mathrm{~h}, 8 \mathrm{~h}$, and $24 \mathrm{~h}$. For each time point the related aggregate distribution in pixel is box plotted, the central mark indicating the median, and bottom and top edges of the box indicating the 25th and 75th percentiles, respectively. $0 \mathrm{mM}$ PBA is shown in blue, $5 \mathrm{mM}$ PBA in green, $20 \mathrm{mM}$ PBA in red and $50 \mathrm{mM}$ PBA in turquoise. 


\section{N-Acetyl-L-Cysteine Data Sets}

\section{C.1 Data Set 2}

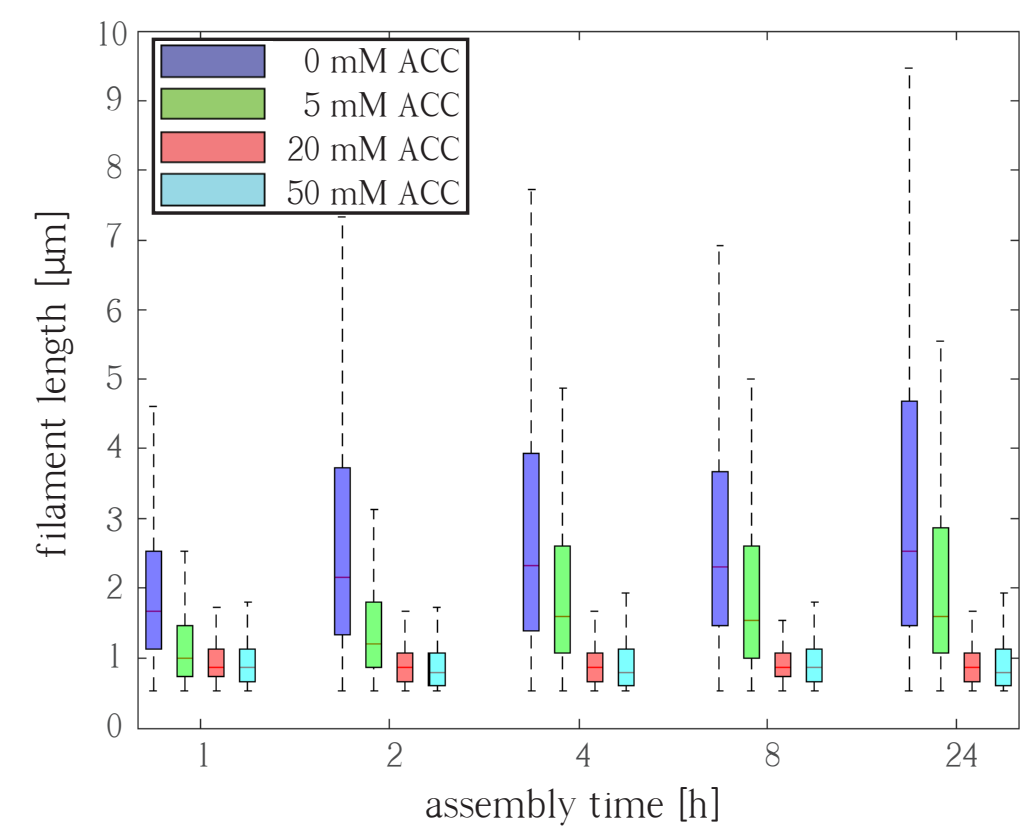

Figure A.5.: Length distributions of vimentin tetramers incubated with different ACC concentrations over $1 \mathrm{~h}, 2 \mathrm{~h}, 4 \mathrm{~h}, 8 \mathrm{~h}$, and $24 \mathrm{~h}$. For each time point the related length distribution in $\mu \mathrm{m}$ is box plotted, the central mark indicating the median, and bottom and top edges of the box indicating the 25th and 75th percentiles, respectively. $0 \mathrm{mM} \mathrm{ACC}$ is shown in blue, $5 \mathrm{mM}$ $A C C$ in green, $20 \mathrm{mM} \mathrm{ACC}$ in red and $50 \mathrm{mM} \mathrm{ACC}$ in turquoise. 


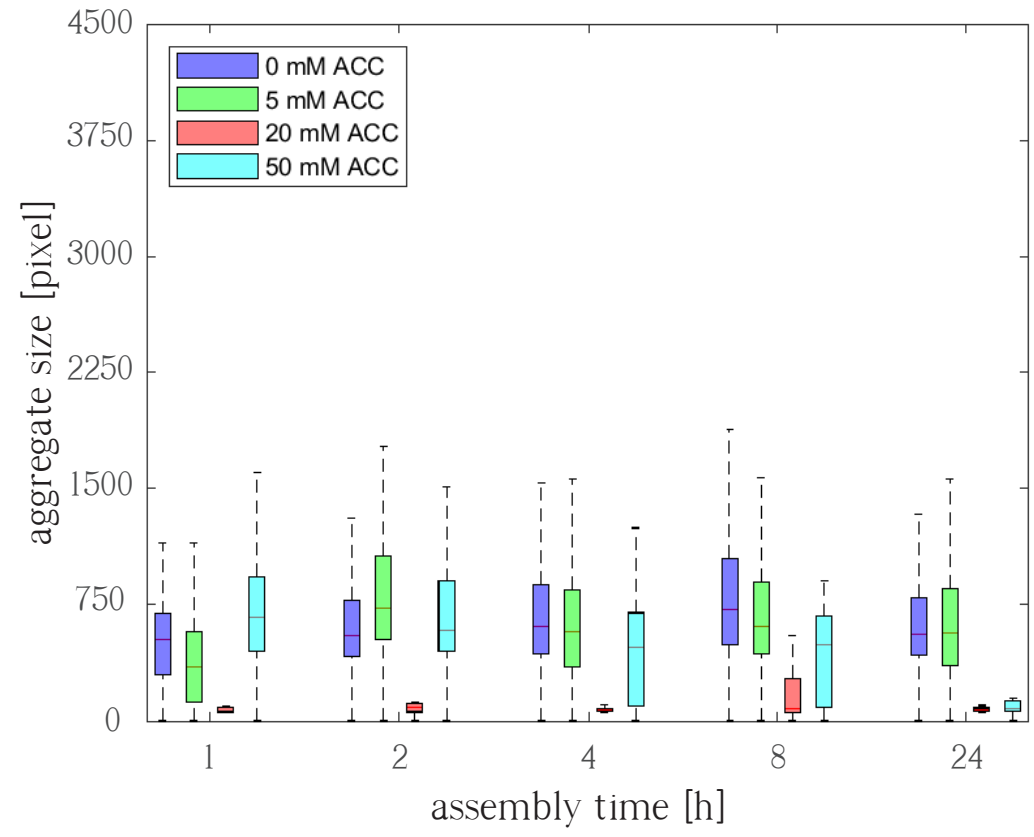

Figure A.6.: Aggregate distributions of vimentin tetramers incubated with different ACC concentrations over $1 \mathrm{~h}, 2 \mathrm{~h}, 4 \mathrm{~h}, 8 \mathrm{~h}$, and $24 \mathrm{~h}$. For each time point the related aggregate distribution in pixel is box plotted, the central mark indicating the median, and bottom and top edges of the box indicating the 25th and 75th percentiles, respectively. $0 \mathrm{mM} \mathrm{ACC}$ is shown in blue, $5 \mathrm{mM}$ ACC in green, $20 \mathrm{mM} \mathrm{ACC}$ in red and $50 \mathrm{mM} \mathrm{ACC}$ in turquoise. 


\section{C.2 Data Set 3}

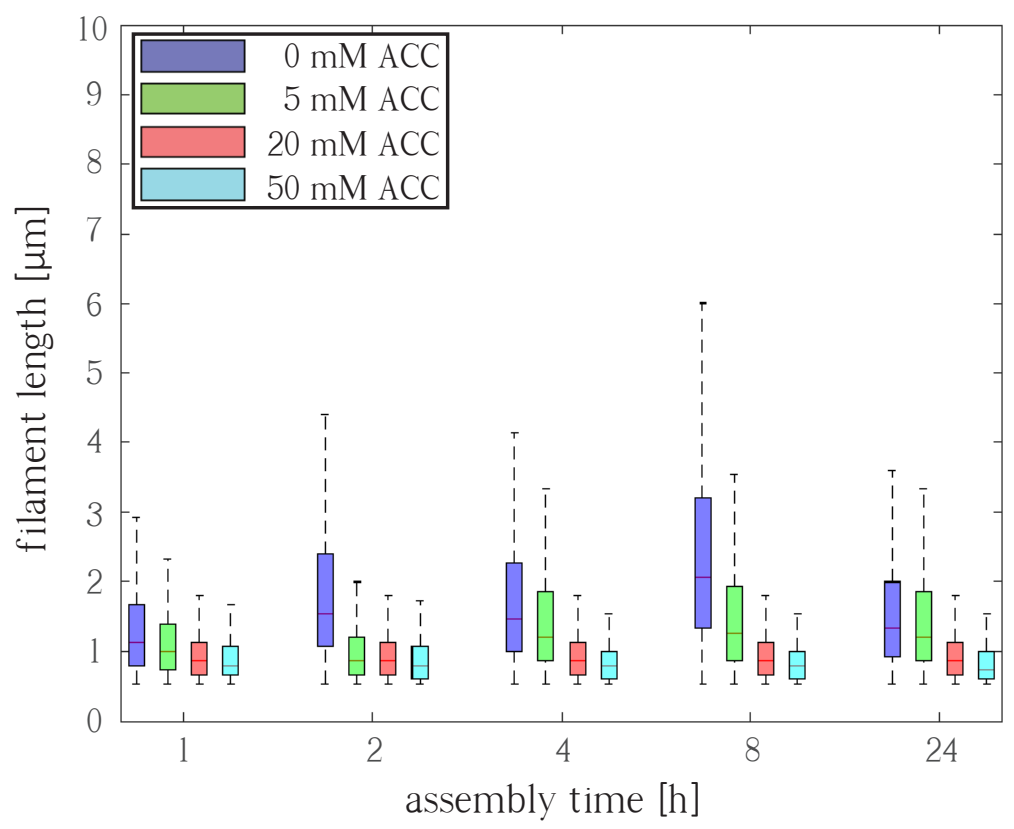

Figure A.7.: Length distributions of vimentin tetramers incubated with different ACC concentrations over $1 \mathrm{~h}, 2 \mathrm{~h}, 4 \mathrm{~h}, 8 \mathrm{~h}$, and $24 \mathrm{~h}$. For each time point the related length distribution in $\mu \mathrm{m}$ is box plotted, the central mark indicating the median, and bottom and top edges of the box indicating the 25th and 75th percentiles, respectively. $0 \mathrm{mM} \mathrm{ACC}$ is shown in blue, $5 \mathrm{mM}$ $A C C$ in green, $20 \mathrm{mM} \mathrm{ACC}$ in red and $50 \mathrm{mM} \mathrm{ACC}$ in turquoise. 


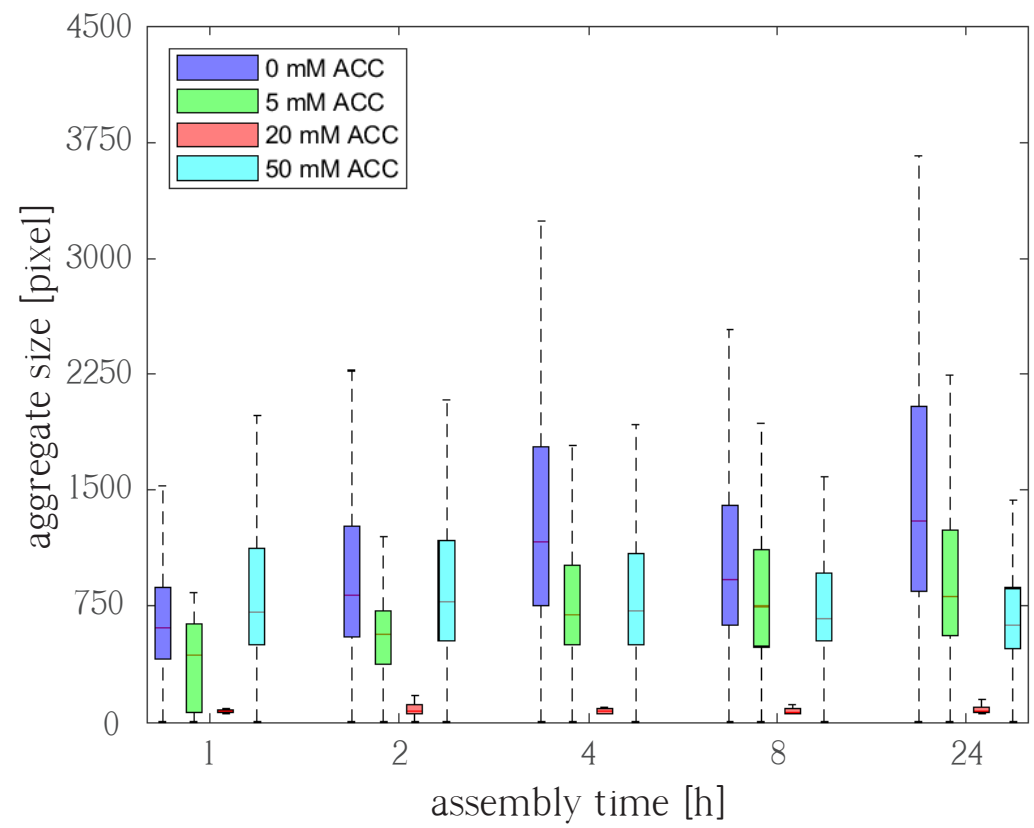

Figure A.8.: Aggregate distributions of vimentin tetramers incubated with different ACC concentrations over $1 \mathrm{~h}, 2 \mathrm{~h}, 4 \mathrm{~h}, 8 \mathrm{~h}$, and $24 \mathrm{~h}$. For each time point the related aggregate distribution in pixel is box plotted, the central mark indicating the median, and bottom and top edges of the box indicating the 25th and 75th percentiles, respectively. $0 \mathrm{mM} \mathrm{ACC}$ is shown in blue, $5 \mathrm{mM}$ ACC in green, $20 \mathrm{mM} \mathrm{ACC}$ in red and $50 \mathrm{mM} \mathrm{ACC}$ in turquoise. 


\section{Bibliography}

1. Alberts, B., Johnson, A., Lewis, J., Morgan, D., Raff, M., Roberts, K. \& Walter, P. Molecular biology of the cell, edn. Garland Science (Taylor and Francis Group, 2015).

2. Jones, R. A. L. Soft Condensed Matter (Oxford University Press, 2002).

3. Powell, B. \& Rogers, G. The Role of Keratin Proteins and Their Genes in the Growth, Structure and Properties of Hair. EXS 78, 59-148 (1997).

4. Herrmann, H., Kreplak, L. \& Aebi, U. Isolation, Characterization, and In Vitro Assembly of Intermediate Filaments. Methods in Cell Biology 78, 3-24 (2004).

5. Guzmán, C., Jeney, S., Kreplak, L., Kasas, S., Kulik, A., Aebi, U. \& Forró, L. Exploring the Mechanical Properties of Single Vimentin Intermediate Filaments by Atomic Force Microscopy. Journal of Molecular Biology 360, 623-630 (2006).

6. Herrmann, H. \& Aebi, U. Intermediate filaments and their associates: multitalented structural elements specifying cytoarchitecture and cytodynamics. Current Opinion in Cell Biology 12, 79-90 (2000).

7. Chang, L. \& Goldman, R. D. Intermediate filaments mediate cytoskeletal crosstalk. Nature Reviews Molecular Cell Biology 5, 601-613 (2004).

8. Viedma-Poyatos, Ã., Pajares, M. A. \& Pérez-Sala, D. Type III intermediate filaments as targets and effectors of electrophiles and oxidants. Redox biology 36, 101582 (2020).

9. Huber, F., Boire, A., López, M. \& Koenderink, G. Cytoskeletal crosstalk: When three different personalities team up. Current Opinion in Cell Biology 32, 39-47 (2015). 
Bibliography

10. Sharma, P., Alsharif, S., Fallatah, A. \& Chung, B. M. Intermediate filaments as effectors of cancer development and metastasis: a focus on keratins, vimentin, and nestin. Cells 8, 497 (2019).

11. Kidd, M. E., Shumaker, D. K. \& Ridge, K. M. The role of vimentin intermediate filaments in the progression of lung cancer. American journal of respiratory cell and molecular biology 50, 1-6 (2014).

12. Omary, M. B. et al. 'IF-pathies': a broad spectrum of intermediate filamentassociated diseases. The Journal of clinical investigation 119, 1756-1762 (2009).

13. Hesse, M., Magin, T. M. \& Weber, K. Genes for intermediate filament proteins and the draft sequence of the human genome. Journal of Cell Science 114, 2569-2575 (2001).

14. Herrmann, H. \& Aebi, U. Intermediate Filaments: Structure and Assembly. Cold Spring Harbor Perspectives in Biology 8 (2016).

15. Conway, J. F. \& Parry, D. A. Intermediate filament structure: 3. Analysis of sequence homologies. International Journal of Biological Macromolecules 10, 7998 (1988).

16. Herrmann, H., Häner, M., Brettel, M., Müller, S., Goldie, K., Fedtke, B., Lustig, A., Franke, W. \& Aebi, U. Structure and assembly properties of the intermediate filament protein vimentin: the role of its head, rod and tail domains. Journal of molecular biology 264, 933-953 (1996).

17. Lowery, J., Kuczmarski, E. R., Herrmann, H. \& Goldman, R. D. Intermediate filaments play a pivotal role in regulating cell architecture and function. Journal of Biological Chemistry 290, 17145-17153 (2015).

18. Mücke, N., Wedig, T., Bürer, A., Marekov, L. N., Steinert, P. M., Langowski, J., Aebi, U. \& Herrmann, H. Molecular and biophysical characterization of assembly-starter units of human vimentin. Journal of molecular biology 340, 97-114 (2004).

19. Pawelzyk, P., Mücke, N., Herrmann, H. \& Willenbacher, N. Attractive interactions among intermediate filaments determine network mechanics in vitro. PloS one 9, e93194 (2014).

20. Winheim, S., Hieb, A., Silbermann, M., Surmann, E.-M., Wedig, T., Herrmann, H., Langowski, J. \& Mücke, N. Deconstructing the Late Phase of Vimentin Assembly by Total Internal Reflection Fluorescence Microscopy (TIRFM). PloS one 6 (2011). 
21. Kirmse, R., Portet, S., Mücke, N., Aebi, U., Herrmann, H. \& Langowski, J. A quantitative kinetic model for the in vitro assembly of intermediate filaments from tetrameric vimentin. Journal of Biological Chemistry 282, 1856318572 (2007).

22. Denz, M., Marschall, M., Herrmann, H. \& Köster, S. Ion type and valency differentially drive vimentin tetramers into intermediate filaments or higher order assemblies. Soft Matter (2020).

23. Downing, D. T. Chemical cross-linking between lysine groups in vimentin oligomers is dependent on local peptide conformations. Proteins: Structure, Function, and Bioinformatics 25, 215-224 (1996).

24. Brennich, M. E., Bauch, S., Vainio, U., Wedig, T., Herrmann, H. \& Köster, S. Impact of ion valency on the assembly of vimentin studied by quantitative small angle X-ray scattering. Soft Matter 10, 2059-2068 (2014).

25. Dammann, C., Herrmann, H. \& Köster, S. Competitive counterion binding regulates the aggregation onset of vimentin intermediate filaments. Israel Journal of Chemistry 56, 614-621 (2016).

26. Nöding, B., Herrmann, H. \& Köster, S. Direct observation of subunit exchange along mature vimentin intermediate filaments. Biophysical journal 107, 2923-2931 (2014).

27. Schepers, A. V., Lorenz, C. \& Köster, S. Tuning intermediate filament mechanics by variation of $\mathrm{pH}$ and ion charges. Nanoscale 12, 15236-15245 (28 2020).

28. Rogers, K. R., Herrmann, H. \& Franke, W. W. Characterization of disulfide crosslink formation of human vimentin at the dimer, tetramer, and intermediate filament levels. Journal of structural biology 117, 55-69 (1996).

29. Mónico, A., Duarte, S., Pajares, M. A. \& Pérez-Sala, D. Vimentin disruption by lipoxidation and electrophiles: Role of the cysteine residue and filament dynamics. Redox biology 23, 101098 (2019).

30. Serres, M. P., Samwer, M., Quang, B. A. T., Lavoie, G., Perera, U., Görlich, D., Charras, G., Petronczki, M., Roux, P. P. \& Paluch, E. K. F-actin interactome reveals vimentin as a key regulator of actin organization and cell mechanics in mitosis. Developmental cell 52, 210-222 (2020). 
Bibliography

31. Schaedel, L., Lorenz, C., Schepers, A. V., Klumpp, S. \& Köster, S. Vimentin Intermediate Filaments Stabilize Dynamic Microtubules by Direct Interactions. bioRxiv (2020).

32. Duarte, S., Viedma-Poyatos, Á., Navarro-Carrasco, E., Martínez, A. E., Pajares, M. A. \& Pérez-Sala, D. Vimentin filaments interact with the actin cortex in mitosis allowing normal cell division. Nature communications 10, 1-19 (2019).

33. Pérez-Sala, D., Oeste, C. L., Martínez, A. E., Carrasco, M. J., Garzón, B. \& Cañada, F. J. Vimentin filament organization and stress sensing depend on its single cysteine residue and zinc binding. Nature Communications 6, 7287 (2015).

34. Tang, H. L., Lung, H. L., Wu, K. C., Le, A.-H. P., Tang, H. M. \& Fung, M. C. Vimentin supports mitochondrial morphology and organization. Biochemical journal 410, 141-146 (2008).

35. Lépinoux-Chambaud, C. \& Eyer, J. Review on intermediate filaments of the nervous system and their pathological alterations. Histochemistry and cell biology 140, 13-22 (2013).

36. Eriksson, J. E., He, T., Trejo-Skalli, A. V., Härmälä-Braskén, A.-S., Hellman, J., Chou, Y.-H. \& Goldman, R. D. Specific in vivo phosphorylation sites determine the assembly dynamics of vimentin intermediate filaments. Journal of cell science 117, 919-932 (2004).

37. Kraxner, J., Lorenz, C., Menzel, J., Parfentev, I., Silbern, I., Denz, M., Urlaub, H., Schwappach, B. \& Köster, S. Post-Translational Modifications Soften Vimentin Intermediate Filaments. Nanoscale (2020).

38. Premchandar, A., Mücke, N., Poznanski, J., Wedig, T., Kaus-Drobek, M., Herrmann, H. \& Dadlez, M. Structural dynamics of the vimentin coiledcoil contact regions involved in filament assembly as revealed by hydrogendeuterium exchange. Journal of Biological Chemistry 291, 24931-24950 (2016).

39. Brennich, M. E., Vainio, U., Wedig, T., Bauch, S., Herrmann, H. \& Köster, S. Mutation-induced alterations of intra-filament subunit organization in vimentin filaments revealed by SAXS. Soft matter 15, 1999-2008 (2019).

40. Janssen, Y., Van Houten, B., Borm, P. \& Mossman, B. Cell and tissue responses to oxidative damage. Laboratory investigation; a journal of technical methods and pathology 69, 261 (1993). 
41. Concannon, C., Gorman, A. \& Samali, A. On the role of Hsp27 in regulating apoptosis. Apoptosis 8, 61-70 (2003).

42. Perng, M., Cairns, L., van den IJssel, P., Prescott, A., Hutcheson, A. \& Quinlan, R. Intermediate filament interactions can be altered by HSP27 and alphaBcrystallin. Journal of Cell Science 112, 2099-2112 (1999).

43. Lee, J.-S., Zhang, M.-H., Yun, E. K., Geum, D., Kim, K., Kim, T.-H., Lim, Y. S. \& Seo, J.-S. Heat shock protein 27 interacts with vimentin and prevents insolubilization of vimentin subunits induced by cadmium. Experimental $\mathcal{E}$ molecular medicine 37, 427-435 (2005).

44. Wettstein, G., Bellaye, P.-S., Micheau, O. \& Bonniaud, P. Small heat shock proteins and the cytoskeleton: An essential interplay for cell integrity? The international journal of biochemistry and cell biology 44, 1680-6 (2012).

45. Kosower, N. S. \& Kosower, E. M. Formation of disulfides with diamide. 143, 264-270 (1987).

46. Brusilow, S. W. Phenylacetylglutamine May Replace Urea as a Vehicle for Waste Nitrogen Excretion. Pediatric Research 29, 147-150 (1991).

47. Iannitti, T. \& Palmieri, B. Clinical and experimental applications of sodium phenylbutyrate. Drugs in R \& D 11, 227-249 (2011).

48. Svechnikova, I., Almqvist, P. \& Ekström, T. HDAC inhibitors effectively induce cell type-specific differentiation in human glioblastoma cell lines of different origin. International journal of oncology 32, 821-7 (2008).

49. Ricobaraza, A., Cuadrado-Tejedor, M., Pérez-Mediavilla, A., Frechilla, D., Del Río, J. \& García-Osta, A. Phenylbutyrate Ameliorates Cognitive Deficit and Reduces Tau Pathology in an Alzheimer's Disease Mouse Model. Neuropsychopharmacology 34, 1721-1732 (2009).

50. Spörrer, M., Prochnicki, A., Tölle, R. C., Nyström, A., Esser, P. R., Homberg, M., Athanasiou, I., Zingkou, E., Schilling, A., Gerum, R., Thievessen, I., Winter, L., Bruckner-Tuderman, L., Fabry, B., Magin, T. M., Dengjel, J., Schröder, R. \& Kiritsi, D. Treatment of keratinocytes with 4-phenylbutyrate in epidermolysis bullosa: Lessons for therapies in keratin disorders. EBioMedicine 44, 502-515 (2019). 
Bibliography

51. Winter, L., Staszewska, I., Mihailovska, E., Fischer, I., Goldmann, W. H., Schröder, R. \& Wiche, G. Chemical chaperone ameliorates pathological protein aggregation in plectin-deficient muscle. The Journal of clinical investigation 124, 1144-1157 (2014).

52. Aldini, G., Altomare, A., Baron, G., Vistoli, G., Carini, M., Borsani, L. \& Sergio, F. N-Acetylcysteine as an antioxidant and disulphide breaking agent: the reasons why. Free radical research 52, 751-762 (2018).

53. Hurst, G. A., Shaw, P. B. \& LeMaistre, C. A. Laboratory and clinical evaluation of the mucolytic properties of acetylcysteine. American Review of Respiratory Disease 96, 962-970 (1967).

54. Noszál, B., Visky, D. \& Kraszni, M. Population, acid- base, and redox properties of N-acetylcysteine conformers. Journal of medicinal chemistry 43, 2176$2182(2000)$.

55. Hémonnot, C. Y., Reinhardt, J., Saldanha, O., Patommel, J., Graceffa, R., Weinhausen, B., Burghammer, M., Schroer, C. G. \& Köster, S. X-rays reveal the internal structure of keratin bundles in whole cells. ACS nano 10, 35533561 (2016).

56. Block, J., Witt, H., Candelli, A., Peterman, E. J., Wuite, G. J., Janshoff, A. \& Köster, S. Nonlinear loading-rate-dependent force response of individual vimentin intermediate filaments to applied strain. Physical review letters 118, 048101 (2017).

57. Dammann, C. \& Köster, S. Dynamics of counterion-induced attraction between vimentin filaments followed in microfluidic drops. Lab Chip 14, 26812687 (2014).

58. Saldanha, O., Brennich, M. E., Burghammer, M., Herrmann, H. \& Köster, S. The filament forming reactions of vimentin tetramers studied in a serialinlet microflow device by small angle x-ray scattering. Biomicrofluidics 10, 024108 (2016).

59. Daw, R. \& Finkelstein, J. Lab Chip. Nature 442, 367-367 (2006).

60. Brennich, M. E., Nolting, J.-F., Dammann, C., Noding, B., Bauch, S., Herrmann, H., Pfohl, T. \& Köster, S. Dynamics of intermediate filament assembly followed in micro-flow by small angle X-ray scattering. Lab Chip 11, 708716 (4 2011). 
61. Salditt, T., Aspelmeier, T. \& Aeffner, S. Biomedical Imaging - Principles of Radiography, Tomography and Medical Physics (De Gruyter, 2017).

62. Jacques, D. A. \& Trewhella, J. Small-angle scattering for structural biologyExpanding the frontier while avoiding the pitfalls. Protein science 19, 642-657 (2010).

63. Evans, R. D. The Atomic Nucleus (McGRAW-Hill Book Company, 1955).

64. Schnablegger, H. \& Singh, Y. The SAXS Guide 3rd (Anton Paar GmbH, 2013).

65. Glatter, O. \& Kratky, O. Small Angle X-ray Scattering (London Academic Press, 1982).

66. Bruus, H. Theoretical Microfluidics (Oxford University Press, 2009).

67. Berger, M. \& Hubbell, J. NIST XCOM: Photon Cross Sections on a Personal Computer tech. rep. 1987.

68. Howells, M., Beetz, T., Chapman, H., Cui, C., Holton, J., Jacobsen, C., Kirz, J., Lima, E., Marchesini, S., Miao, H., Sayre, D., Shapiro, D., Spence, J. \& Starodub, D. An assessment of the resolution limitation due to radiationdamage in X-ray diffraction microscopy. Journal of Electron Spectroscopy and Related Phenomena 170, 4-12 (2009).

69. Thomson, J. J. Conduction of Electricity Through Gases (University Press, Cambridge, England), 2nd edn, 1906).

70. Compton, A. H. A quantum theory of the scattering of X-rays by light elements. Phys. Rev. (1923).

71. Harroun, T., Wignall, G., Fitter, J., Gutberlet, T. \& Katsaras, J. Neutron Scattering for Biology. Neutron Scattering in Biology, 1-18 (2006).

72. Falta, J. \& Möller, T. Forschung mit Synchrotronstrahlung (Vieweg + Teubner, 2010).

73. Guinier, A., Fournet, G. \& Yudowitch, K. L. Small-angle scattering of X-rays (1955).

74. Baruchel, J., Hodeau, J., Lehmann, M., Regnard, J. \& Schlenker, C. Neutron and Synchrotron Radiation for Condensed Matter Studies: Volume 1: Theory, Instruments and Methods (Springer Berlin Heidelberg, 1993).

75. Rayleigh, L. Form factor of a homogenous sphere. Proc. R. Soc. London, Ser. A 84, 25-38 (1911). 
Bibliography

76. Pedersen, J. S. Analysis of small-angle scattering data from colloids and polymer solutions: modeling and least-squares fitting. Advances in Colloid and Interface Science 70, 171-210 (1997).

77. Pedersen, J. S. Form factors of block copolymer micelles with spherical, ellipsoidal and cylindrical cores. Journal of applied crystallography 33, 637-640 (2000).

78. Huang, T., Toraya, H., Blanton, T. \& Wu, Y. X-ray powder diffraction analysis of silver behenate, a possible low-angle diffraction standard. Journal of applied crystallography 26, 180-184 (1993).

79. Mertens, H. D. \& Svergun, D. I. Structural characterization of proteins and complexes using small-angle X-ray solution scattering. Journal of structural biology 172, 128-141 (2010).

80. Feigin, L. A. \& Svergun, D. I. Structure Analysis by Small-Angle X-Ray and Neutron Scattering 1st ed., 350 (Plenum Press, New York, 1987).

81. Kikhney, A. G. \& Svergun, D. I. A practical guide to small angle X-ray scattering (SAXS) of flexible and intrinsically disordered proteins. FEBS letters 589, 2570-2577 (2015).

82. Moore, P. B. Small-angle scattering. Information content and error analysis. Journal of Applied Crystallography 13, 168-175 (1980).

83. Squires, T. M. \& Quake, S. R. Microfluidics: Fluid physics at the nanoliter scale. Rev. Mod. Phys. 77, 977-1026 (3 2005).

84. Robert, A., Rossow, M. J., Hookway, C., Adam, S. A. \& Gelfand, V. I. Vimentin filament precursors exchange subunits in an ATP-dependent manner. Proceedings of the National Academy of Sciences 112, E3505-E3514 (2015).

85. Koblmiller, C. Edler Meilenstein. Chemie Technik, 14-16 (2000).

86. Impact of simulated biological aging on physicochemical and biocompatibility properties of cyclic olefin copolymers. Materials Science and Engineering: C 97, 377-387 (2019).

87. Shin, J. Y., Park, J. Y., Liu, et al. Chemical structure and physical properties of cyclic olefin copolymers (IUPAC Technical Report). Pure and Applied Chemistry 77, pp. 801-814 (2005). 
88. Donner, M., Fernandes, M. \& Kaminsky, W. Synthesis of Copolymers with Sterically Hindered and Polar Monomers. Macromolecular Symposia 236, 193202 (2006).

89. Shin, J. Y., Park, J. Y., Liu, C., He, J. \& Kim, S. C. Chemical structure and physical properties of cyclic olefin copolymers (IUPAC technical report). Pure and Applied Chemistry 77, 801-814 (2005).

90. Guha, S., Perry, S. L., Pawate, A. S. \& Kenis, P. J. Fabrication of X-ray compatible microfluidic platforms for protein crystallization. Sens Actuators B Chem 174, 1-9 (2012).

91. Condon, E. A theory of intensity distribution in band systems. Physical Review 28, 1182 (1926).

92. Jablonski, A. Efficiency of anti-Stokes fluorescence in dyes. Nature 131, 839840 (1933).

93. Stokes, G. G. XXX. On the change of refrangibility of light. Philosophical Transactions of the Royal Society of London 142, 463-562 (1852).

94. Lichtman, J. W. \& Conchello, J.-A. Fluorescence microscopy. Nature methods 2, 910-919 (2005).

95. Block, J., Witt, H., Candelli, A., Danes, J. C., Peterman, E. J. G., Wuite, G. J. L., Janshoff, A. \& Köster, S. Viscoelastic properties of vimentin originate from nonequilibrium conformational changes. Science Advances 4 (2018).

96. Dhouib, K., Malek, C. K., Pfleging, W., Gauthier-Manuel, B., Duffait, R., Thuillier, G., Ferrigno, R., Jacquamet, L., Ohana, J., Ferrer, J.-L., et al. Microfluidic chips for the crystallization of biomacromolecules by counterdiffusion and on-chip crystal X-ray analysis. Lab on a Chip 9, 1412-1421 (2009).

97. Denz, M., Brehm, G. \& et al. Cyclic olefin copolymer as an X-ray compatible material for microfluidic devices. Lab Chip 18, 171-178 (1 2018).

98. Park, H. Y., Qiu, X., Rhoades, E., Korlach, J., Kwok, L. W., Zipfel, W. R., Webb, W. W. \& Pollack, L. Achieving uniform mixing in a microfluidic device: hydrodynamic focusing prior to mixing. Analytical chemistry 78, 44654473 (2006).

99. TOPAS COC Datasheets (TOPAS Advanced Polymers GmbH, 2019).

100. ChipShop, M. Lab-on-a-Chip Catalogue (microfluidic ChipShop, 2016). 
101. Urbani, R. B. Dynamics in Microfluidics Measured by X-Ray Scattering Techniques PhD thesis (Philosophisch-Naturwissenschaftliche Fakultät der Universität Basel, 2015).

102. Henrich, B., Bergamaschi, A., Broennimann, C., Dinapoli, R., Eikenberry, E., Johnson, I., Kobas, M., Kraft, P., Mozzanica, A. \& Schmitt, B. PILATUS: A single photon counting pixel detector for X-ray applications. Nuclear Instruments and Methods in Physics Research Section A: Accelerators, Spectrometers, Detectors and Associated Equipment 607, 247-249 (2009).

103. Mücke, N., Kämmerer, L., Winheim, S., Kirmse, R., Krieger, J., Mildenberger, M., Baßler, J., Hurt, E., Goldmann, W. H., Aebi, U., et al. Assembly kinetics of vimentin tetramers to unit-length filaments: A stopped-flow study. Biophysical journal 114, 2408-2418 (2018).

104. Perego, E. Studying Molecular Interactions under Flow with Fluorescence Fluctuation Spectroscopy PhD thesis (Georg-August-Universität Göttingen, 2020).

105. Bronstein, I. N. \& Semendjajew, K. A. Taschenbuch der Mathematik (Verlag Harri Deutsch, 1981).

106. Skou, S., Gillilan, R. E. \& Ando, N. Synchrotron-based small-angle X-ray scattering of proteins in solution. Nature protocols 9, 1727-1739 (2014).

107. Mücke, N., Kreplak, L., Kirmse, R., Wedig, T., Herrmann, H., Aebi, U. \& Langowski, J. Assessing the flexibility of intermediate filaments by atomic force microscopy. Journal of molecular biology 335, 1241-1250 (2004).

108. Kreplak, L. \& Fudge, D. Biomechanical properties of intermediate filaments: from tissues to single filaments and back. Bioessays 29, 26-35 (2007).

109. Saldanha, O., Graceffa, R., Hémonnot, C. Y. J., Ranke, C., Brehm, G., Liebi, M., Marmiroli, B., Weinhausen, B., Burghammer, M. \& Köster, S. Rapid Acquisition of X-Ray Scattering Data from Droplet-Encapsulated Protein Systems. ChemPhysChem 18, 1220-1223 (2017).

110. Van der Linden, P. J., Popov, A. M. \& Pontoni, D. Accurate and rapid 3D printing of microfluidic devices using wavelength selection on a DLP printer. Lab Chip 20, 4128-4140 (2020).

111. Barrett, R., Faucon, M., Lopez, J., Cristobal, G., Destremaut, F., Dodge, A., Guillot, P., Laval, P., Masselon, C. \& Salmon, J.-B. X-ray microfocussing combined with microfluidics for on-chip X-ray scattering measurements. Lab Chip 6, 494-499 (2006). 
112. Duffy, D. C., McDonald, J. C., Schueller, O. J. \& Whitesides, G. M. Rapid prototyping of microfluidic systems in poly (dimethylsiloxane). Analytical chemistry 70, 4974-4984 (1998).

113. Norland Optical Adhesive 81 https : / / www . norlandprod.com/adhesives / NOA81.html. (accessed: 12.01.2021).

114. Zanic, M. in The Mitotic Spindle 47-61 (Springer, 2016).

115. Kapoor, V., Hirst, W. G., Hentschel, C., Preibisch, S. \& Reber, S. MTrack: automated detection, tracking, and analysis of dynamic microtubules. Scientific reports 9, 1-12 (2019).

116. Schneider, M., Al-Shaer, A. \& Forde, N. R. AutoSmarTrace: Automated Chain Tracing and Flexibility Analysis of Biological Filaments. bioRxiv (2021).

117. Denz, M. Influence of Ions on the Assembly of Vimentin Intermediate Filaments PhD thesis (Georg-August-Universität Göttingen, 2020). 



\section{Danksagung - Acknowledgments}

An dieser Stelle möchte ich meinen Dank an alle aussprechen, die mich bei dieser Arbeit unterstützt haben. Zuallererst möchte ich Sarah Köster danken, dass sie mir die die Möglichkeit gegeben hat diese Arbeit in ihrer Gruppe anzufertigen. Die umfassende Betreuung und Förderung, sowie die vielfältigen Möglichkeiten meine Arbeit an Konferenzen vorzustellen, an Messzeiten teilzunehmen und Kontakte zu knüpfen hat wesentlich zum Gelingen dieser Arbeit beigetragen. Sie hat sich stets für wissenschaftliche Diskussionen Zeit genommen und hatte immer ein offenes Ohr für neue Ideen. Weiterhin gilt mein Dank Tim Salditt. Seine Begeisterung für das Thema meiner Dissertation und die fachlichen Diskussionen, nicht nur während meiner TAC Treffen, haben meine Arbeit wesentlich vorangetrieben. Für die Übernahme des Zweitgutachtens sei Ihm an dieser Stelle nochmals gedankt. Thomas Burg danke ich für die fachliche Begleitung meiner Promotion und seine Anregungen in meinen TAC Treffen. Alexander Egner, Stefan Klumpp und Simone Techert danke ich für die Bereitschaft meiner Prüfungskommission anzugehören. Susanne Bauch möchte ich für das Vimentin und die geduldigen Antworten auf die vielen kleinen Fragen im Labor danken. Danke Jan Goeman für das Lösen von IT-Problemen und Peter Luley für die Hilfe bei technischen Fragen. Ana Diaz, Manfred Burghammer, Markus Osterhoff und Michael Sprung danke ich für die Betreuung und Unterstützung bei den Messzeiten. Christine Wilke-Feist, Kerstin Pluschke, Michaela Ständer, Eva Hetzel and Sabine Balder danke ich für die administrative Unterstützung.

Weiter möchte ich mich bei allen aktuellen und früheren Mitgliedern der AG Köster und den Kollegen und Kolleginnen im IRP bedanken für die Selbstverständlichkeit mit der Hilfe angeboten wird, zahllose Diskussionen und die gute Atmosphäre. Besonders danken möchte ich Andrew Wittmeier, für deine Hilfe 


\section{Bibliography}

bei den kleineren und größeren Problem zu jeder Tages und Nacht Zeit sei dir gedankt. Dem X-Ray Team, Manuela Denz, Chiara Cassini, Andrew Wittmeier, Jan-Philipp Burchert und Sophie-Charlotte August danke ich die für Unterstützung vor und während der Messzeiten, die vielen Diskussionen und die schönen Momente. Danke Manuela Denz für deinen fachlichen Rat, die Gespräche und das Umsorgen der XEUSS. Danke Chiara Cassini für deine Hilfe im Labor und all die Diskussionen auf Messzeiten zu später Stunde. Eleonora Perego danke ich dafür immer eine Lösung zu haben, Anna Schepers, Julia Kraxner und Charlotta Lorenz sei gedankt für die Unterstützung im Labor, den Laborprotokollen und den Diskussionen über Vimentin. Heidi Somsel danke ich für ihre Unterstützung innerhalb und außerhalb der Universität. Danke Jan-Philipp Burchert für deine Expertise beim Programmieren. Für die konstruktive Rückmeldung während der Entstehung dieser Arbeit sein Andrew Wittmeier, Eleonora Perego und Heidi Somsel herzlich gedankt.

Mein besonderer Dank gilt meiner Familie und meinen Freuden. Ihr wart in dieser Zeit eine unbeschreibliche Stütze. Ihr habt mich in jeder nur denkbaren Weise unterstützt und ermutigt diesen Weg zu gehen. Danke, dass ihr mich seit jeher liebevoll begleitet. Pauline dir danke ich insbesondere für dein Verständnis, deine Unterstützung und Ermutigungen und mit Léonie zusammen für jedes Lächeln. 


\section{List of Publications}

1. Saldanha, O., Brennich, M. E., Burghammer, M., Herrmann, H. \& Köster, S. The filament forming reactions of vimentin tetramers studied in a serialinlet microflow device by small angle x-ray scattering. Biomicrofluidics 10, 024108 (2016).

2. Denz, M., Brehm, G. \& et al. Cyclic olefin copolymer as an X-ray compatible material for microfluidic devices. Lab Chip 18, 171-178 (1 2018).

3. Ishigami, I., Lewis-Ballester, A., Echelmeier, A., Brehm, G., Zatsepin, N. A., Grant, T. D., Coe, J. D., Lisova, S., Nelson, G., Zhang, S., et al. Snapshot of an oxygen intermediate in the catalytic reaction of cytochrome c oxidase. Proceedings of the National Academy of Sciences 116, 3572-3577 (2019).

4. Echelmeier, A., Kim, D., Cruz Villarreal, J., Coe, J., Quintana, S., Brehm, G., Egatz-Gomez, A., Nazari, R., Sierra, R. G., Koglin, J. E., et al. 3D printed droplet generation devices for serial femtosecond crystallography enabled by surface coating. Journal of applied crystallography 52, 997-1008 (2019).

5. Brehm, G., Echelmeier, A., Coe, J., Conrad, C. E., Nelson, G., Villarreal, J. C., Weierstall, U., Spence, J., Köster, S., Fromme, P., et al. Combined hydrodynamic mixer and gas dynamic virtual nozzle devices for serial femtosecond crystallography in 21st International Conference on Miniaturized Systems for Chemistry and Life Sciences, MicroTAS 2017 (2020), 1495-1496.

6. Epple, R., Krüger, D., Berulava, T., Brehm, G., Islam, R., Köster, S. \& Fischer, A. The Coding And Small-Non-Coding Hippocampal Synaptic RNAome. bioRxiv (2020).

7. Gisriel, C., Coe, J., Letrun, R., Yefanov, O. M., Luna-Chavez, C., Stander, N. E., Lisova, S., Mariani, V., Kuhn, M., Aplin, S., et al. Membrane protein 


\section{\begin{tabular}{l|l} 
Appendix & LIST OF PUBLICATIONS
\end{tabular}}

megahertz crystallography at the European XFEL. Nature communications 10, 1-11 (2019).

8. Echelmeier, A., Villarreal, J. C., Messerschmidt, M., Kim, D., Coe, J. D., Thifault, D., Botha, S., Egatz-Gomez, A., Gandhi, S., Brehm, G., et al. Segmented flow generator for serial crystallography at the European $X$-ray free electron laser. Nature communications 11, 1-10 (2020).

9. Komorowski, K., Schaeper, J., Sztucki, M., Sharpnack, L., Brehm, G., Köster, S. \& Salditt, T. Vesicle adhesion in the electrostatic strong-coupling regime studied by time-resolved small-angle X-ray scattering. Soft matter 16, 4142 4154 (2020).

10. Cassini, C., Wittmeier, A., Brehm, G., Denz, M., Burghammer, M. \& Köster, S. Large field-of-view scanning small-angle $X$-ray scattering of mammalian cells. Journal of Synchrotron Radiation 27 (2020). 